Prepared in cooperation with the

SWEETWATER AUTHORITY

\title{
Water- and Air-Quality Monitoring of the Sweetwater Reservoir Watershed, San Diego County, California- Phase One Results, Continued, 2001-2003
}

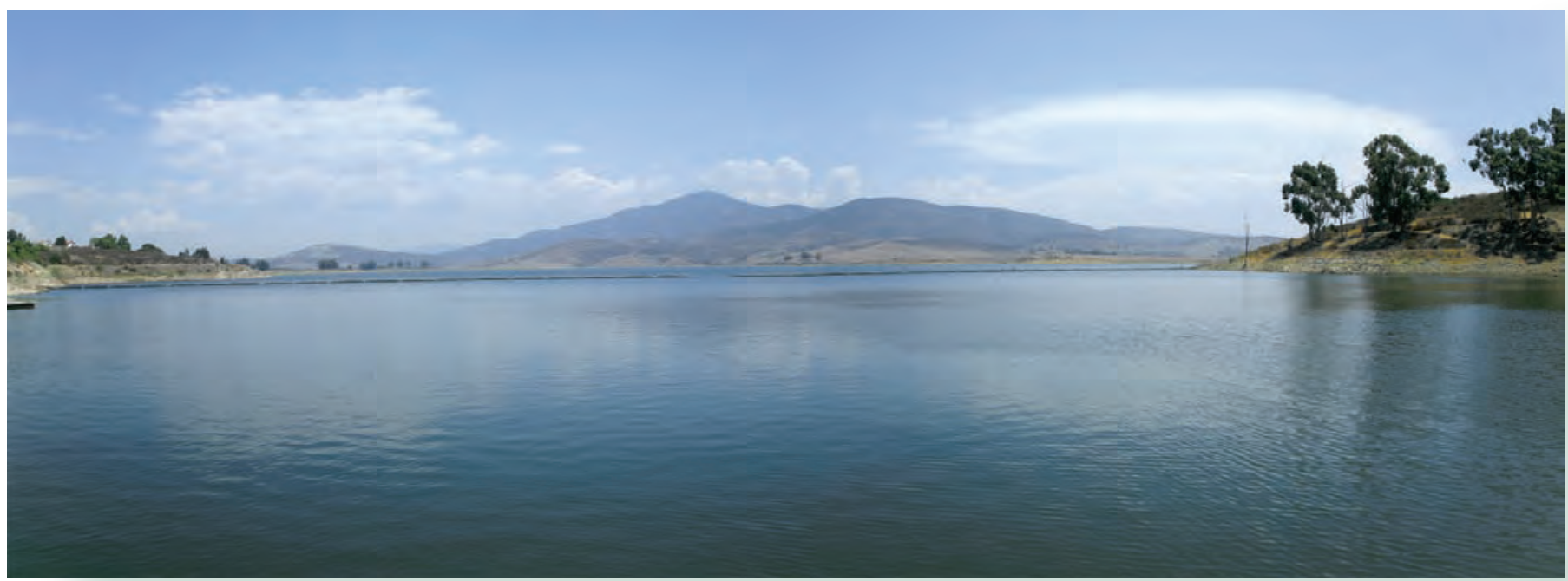

Data Series Report 347 



\section{Water- and Air-Quality Monitoring of Sweetwater Reservoir Watershed, San Diego County, California-Phase One Results Continued, 2001-2003}

By Gregory 0. Mendez, William T. Foreman, Andrew Morita, and Michael S. Majewski

Prepared in cooperation with the Sweetwater Authority

Data Series 347 


\section{U.S. Department of the Interior DIRK KEMPTHORNE, Secretary}

\section{U.S. Geological Survey \\ Mark D. Myers, Director}

\section{U.S. Geological Survey, Reston, Virginia: 2008}

For product and ordering information:

World Wide Web: http://www.usgs.gov/pubprod

Telephone: 1-888-ASK-USGS

For more information on the USGS--the Federal source for science about the Earth, its natural and living resources, natural hazards, and the environment:

World Wide Web: http://www.usgs.gov

Telephone: 1-888-ASK-USGS

Any use of trade, product, or firm names is for descriptive purposes only and does not imply endorsement by the U.S. Government.

Although this report is in the public domain, permission must be secured from the individual copyright owners to reproduce any copyrighted materials contained within this report.

Suggested citation:

Mendez, G.O., Foreman, W.T., Morita, Andrew, and Majewski, M.S., 2008, Water- and air-quality monitoring of Sweetwater Reservoir Watershed, San Diego County, California-Phase One Results Continued, 2001-2003: U.S. Geological Survey Data Series 347, 158 p. 


\section{Contents}

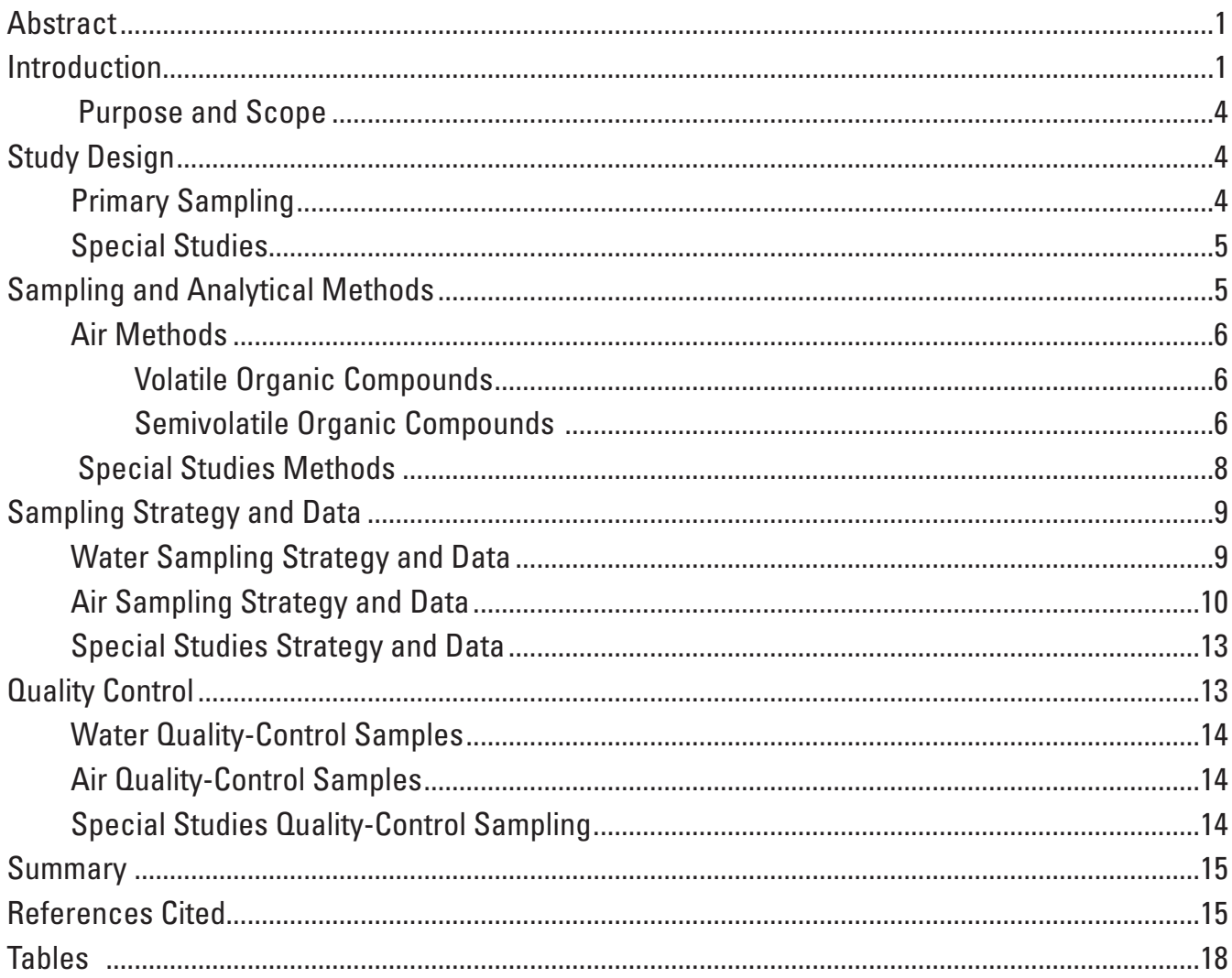

\section{Figures}

Figure 1. The general vicinity of Sweetwater and Loveland Reservoirs and surrounding watershed, San Diego County, California .................................. 2

Figure 2. Sweetwater Reservoir and SR125 alignment, San Diego County, California ........ 3

Figure 3. Sampling sites at Loveland Reservoir, San Diego County, California .............. 5

Figure 4. (A) Concentrations in air of methyl-tert-butyl ether (MTBE), benzene, and toluene at the air sampling site, and concentrations in air and in water of $(B)$ methyl-tert-butyl ether, $(C)$ benzene, and $(D)$ toluene at Sweetwater Reservoir (SWR) near the pump tower, and SWR center of minimum pool, San Diego County, California

Figure 5. Concentrations of simazine in finished water at site SWR-08, in imported water at site SWR-09, and near the pump tower at Sweetwater Reservoir, San Diego County, California 


\section{Tables}

Table 1. Site identification numbers, site numbers, and corresponding site names, Sweetwater and Loveland Reservoirs, San Diego County, California

Table 2. Water-quality depth-profile data for dissolved oxygen, $\mathrm{pH}$, specific conductance, and temperature for each sampling site and period for the Sweetwater and Loveland Reservoirs, San Diego County, California

Table 3A. Volatile organic compounds analyzed in whole-water samples from the Sweetwater Reservoir watershed, San Diego County, California

Table 3B. Analytical results for volatile organic compounds detected in whole-water samples from the Sweetwater Reservoir watershed, San Diego County, California

Table 4A. Polycyclic aromatic hydrocarbon compounds analyzed in whole-water samples from the Sweetwater Reservoir watershed, San Diego County, California

Table 4B. Analytical results for polycyclic aromatic hydrocarbon concentrations in whole-water samples from the Sweetwater Reservoir watershed, San Diego County, California

Table 5A. Pesticide compounds analyzed in filtered-water samples, using U.S.Geological Survey's National Water Quality Laboratory schedule 2001, from the Sweetwater Reservoir watershed, San Diego County, California

Table 5B. Analytical results for pesticides detected in filtered water samples, using U.S. Geological Survey's National Water Quality Laboratory Schedule 2001, from the Sweetwater Reservoir watershed, San Diego, California

Table 6A. Pesticide compounds analyzed in filtered-water samples, using U.S. Geological Survey's National Water Quality Laboratory schedule 2002, from the Sweetwater Reservoir watershed, San Diego County, California

Table 6B. Analytical results for pesticide compounds detected in filtered-water samples, using U.S. Geological Survey's National Water Quality Laboratory schedule 2002, from the Sweetwater Reservoir watershed, San Diego County, California

Table 7A. Pesticide compounds analyzed in filtered-water samples, using U.S. Geological Survey's National Water Quality Laboratory schedule 2003, from the Sweetwater Reservoir watershed, San Diego County, California

Table 7B. Analytical results for pesticide compounds detected in filtered-water samples, using U.S. Geological Survey's National Water Quality Laboratory schedule 2003, from the Sweetwater Reservoir watershed, San Diego County, California

Table 8A. Pesticide compounds analyzed in filtered-water samples, using U.S. Geological Survey's National Water Quality Laboratory schedule 2060, from the Sweetwater Reservoir watershed, San Diego County, California

Table 8B. Analytical results for pesticide compounds detected in filtered-water samples, using U.S. Geological Survey's National Water Quality Laboratory Schedule 2060, from the Sweetwater Reservoir watershed, San Diego County, California 
Table 9A. Analytical results for volatile organic compounds in air with low breakthrough volumes from the Sweetwater Reservoir atmospheric site, San Diego County,

California

Table 9B. Analytical results for volatile organic compounds in air with high breakthrough volumes from the Sweetwater Reservoir atmospheric site, San Diego County, California

Table 10A. Polycyclic aromatic hydrocarbon (PAH) and alkylated PAH compounds analyzed in air samples collected from the Sweetwater Reservoir atmospheric site, San Diego County, California

Table 10B. Analytical results for polycyclic aromatic hydrocarbon (PAH) and alkylated PAH concentrations in air from the Sweetwater Reservoir atmospheric site, San Diego County, California

Table 11A. Pesticide compounds analyzed using method Airpest001 for air samples collected from the Sweetwater Reservoir atmospheric site, San Diego County, California

Table 11B. Analytical results for pesticide concentrations detected using method Airpest001 for air samples collected from the Sweetwater Reservoir atmospheric site, San Diego County, California

Table 12A. Pesticide compounds analyzed using method Airpest002 for air samples collected from the Sweetwater Reservoir atmospheric site, California

Table 12B. Analytical results for pesticide concentrations detected using method Airpest002 for air samples collected from the Sweetwater Reservoir atmospheric site, San diego County, California

Table 13A. Pesticide compounds analyzed using method Airpest003 for air samples collected from the Sweetwater Reservoir atmospheric site, San Diego County, California

Table 13B. Analytical results for pesticide concentrations detected using method Airpest003 for air samples collected from the Sweetwater Reservoir atmospheric site, San Diego County, California

Table 14A. Analytical results for special study of trace metals in whole-water samples collected from the Sweetwater Reservoir watershed, San Diego County, California

Table 14B. Analytical results for special study of trace metals in filtered-water samples collected from the Sweetwater Reservoir watershed, San Diego County, California

Table 15A. Anthropogenic indicator compounds analyzed in filtered-water samples collected from the Sweetwater Reservoir watershed, San Diego County, California

Table 15B. Analytical results from the special study of anthropogenic indicator compounds detected in filtered-water samples collected from the Sweetwater Reservoir watershed, San Diego County, California

Table 16. Analytical results from the special study for pharmaceutical compounds in filtered-water samples collected from Sweetwater Reservoir watershed, San Diego County, California 
Table 17. Quality-control results for volatile organic compound concentrations in wholewater samples collected from the Sweetwater Reservoir watershed, San Diego County,California

Table 18. Quality-control results for pesticide concentrations in filtered-water samples, analyzed using U.S. Geological Survey's National Water Quality Laboratory schedule 2001, from Loveland Reservoir, San Diego County, California 118

Table 19. Quality-control results for pesticide concentrations in filtered-water samples, analyzed using U.S. Geological Survey's National Water Quality Laboratory schedule 2060, from Sweetwater Reservoir, San Diego County, California

Table 20A. Quality-control replicate results for volatile organic compounds with low breakthrough volumes from the Sweetwater Reservoir atmospheric site, San Diego County, California .

Table 20B. Quality-control replicate results for volatile organic compounds with high breakthrough volumes from the Sweetwater Reservoir atmospheric site, San Diego County, California ....

Table 21A. Quality-control results for volatile organic compounds with low breakthrough volumes in laboratory spikes for air samples collected at the Sweetwater Reservoir atmospheric site, San Diego County, California

Table 21B. Quality-control results for volatile organic compounds with low breakthrough volumes in travel blanks for air samples collected at the Sweetwater Reservoir atmospheric site, San Diego County, California .....

Table 21C. Quality-control results for volatile organic compounds with low breakthrough volumes in lot blanks processed with air samples collected at the Sweetwater Reservoir atmospheric site, San Diego County, California

Table 21D. Quality-control results for volatile organic compounds with low breakthrough volumes in laboratory blanks for air samples collected at the Sweetwater Reservoir atmospheric site, San Diego County, California

Table 22A. Quality-control results for volatile organic compounds with high breakthrough volumes in laboratory spikes for air samples collected at the Sweetwater Reservoir atmospheric site, San Diego County, California

Table 22B. Quality-control results for volatile organic compounds with high breakthrough volumes in travel blanks for air samples collected at Sweetwater Reservoir atmospheric site at Sweetwater Dam, San Diego County, California

Table 22C. Quality-control detections for volatile organic compounds with high breakthrough volumes in lot blanks for air samples collected at the Sweetwater Reservoir atmospheric site, San Diego County, California

Table 22D. Quality-control detections for volatile organic compounds with high breakthrough volumes in laboratory blanks for air samples collected at the Sweetwater Reservoir atmospheric site, San Diego County, California

Table 23A. Quality-control results for laboratory and field reagent blanks and laboratory solvent blanks for polycyclic aromatic hydrocarbon (PAH) compound concentrations from the Sweetwater Reservoir atmospheric site, San Diego County, California

Table 23B. Quality-control results for polycyclic aromatic hydrocarbon (PAH) compound laboratory reagent-spikes for air samples collected at the Sweetwater Reservoir atmospheric site, San Diego County, California 
Table 24A. Quality-control results for pesticide blanks using U.S. Geological Survey's National Water Quality Laboratory method Airpest001 for air samples collected at the Sweetwater Reservoir atmospheric site, San Diego County, California ...... 144

Table 24B. Quality-control results for pesticide laboratory reagent-spikes using U.S. Geological Survey's National Water Quality Laboratory method Airpest001 for air samples collected at the Sweetwater Reservoir atmospheric site, San Diego

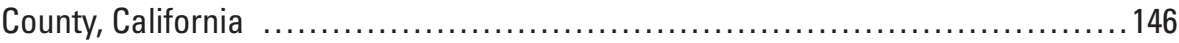

Table 25A. Quality-control laboratory blank results using pesticide method Airpest002 for air samples collected at the Sweetwater Reservoir atmospheric site, San Diego County, California

Table 25B. Quality-control results for pesticide laboratory reagent spikes using method Airpest002 for samples collected at the Sweetwater Reservoir atmospheric site, San Diego County, California

Table 26A. Quality-control laboratory blank results using pesticides method Airpest003 for air samples collected at the Sweetwater Reservoir atmospheric site, San Diego County, California

Table 26B. Quality-control laboratory reagent-spike results using pesticide method Airpest003 for air samples collected at the Sweetwater Reservoir atmospheric site, San Diego County, California

Table 27A. Quality-control laboratory reagent-water blank results for the special study of pharmaceutical compounds in the Sweetwater Reservoir watershed, San Diego County, California

Table 27B. Quality-control laboratory reagent-water spike results for the special study of pharmaceutical compounds in the Sweetwater Reservoir watershed, San Diego County, California 


\section{Abbreviations and Acronyms}

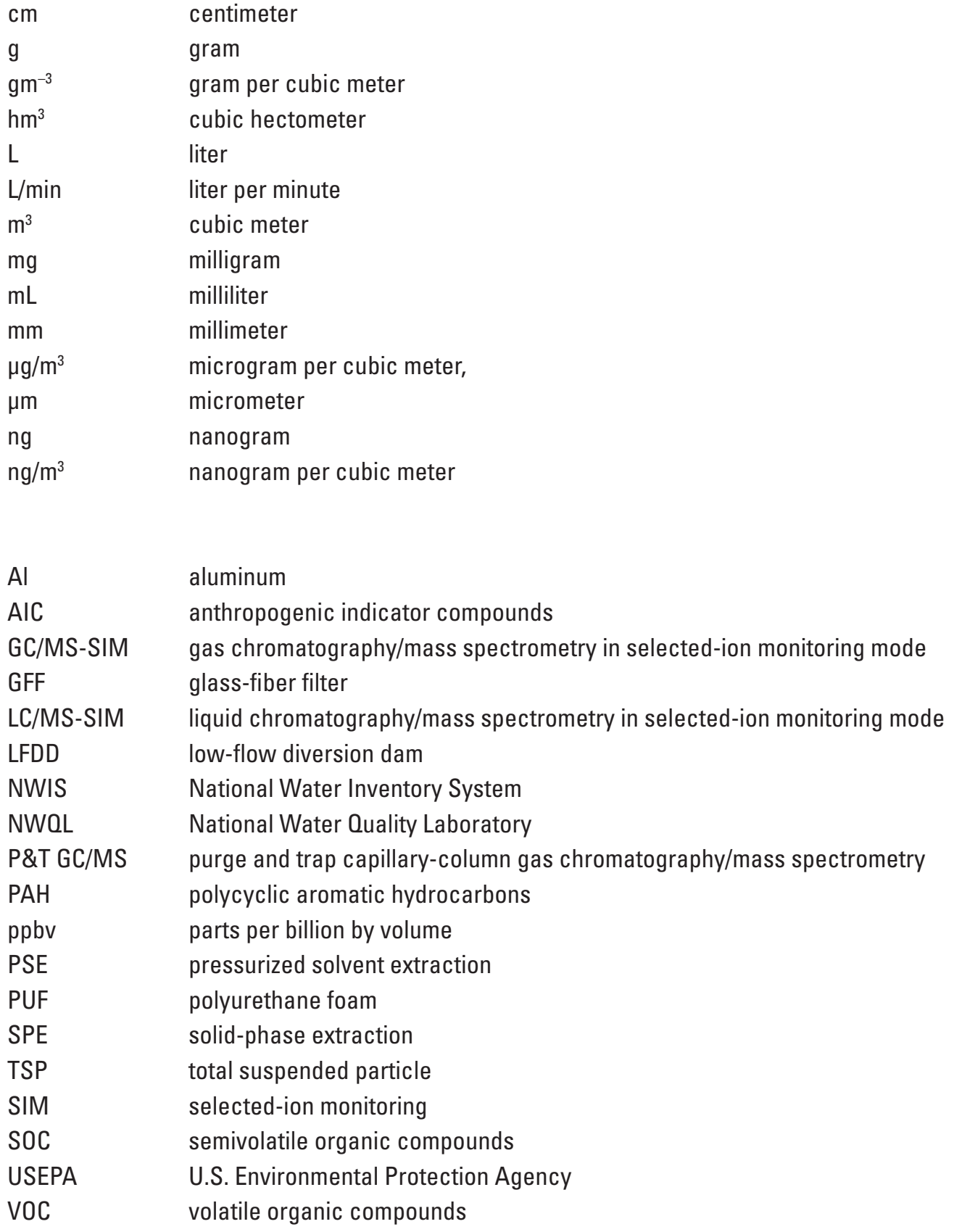

Specific conductance is given in microsiemens per centimeter at 25 degrees Celsius $\left(\mu \mathrm{S} / \mathrm{cm}\right.$ at $\left.25^{\circ} \mathrm{C}\right)$.

Temperature in degrees Celsius $\left({ }^{\circ} \mathrm{C}\right)$ may be converted to degrees Fahrenheit $\left({ }^{\circ} \mathrm{F}\right)$ as follows:

$$
{ }^{\circ} \mathrm{F}=\left(1.8 x^{\circ} \mathrm{C}\right)+32
$$




\title{
Water- and Air-Quality Monitoring of Sweetwater Reservoir Watershed, San Diego County, California- Phase One Results Continued, 2001-2003
}

\author{
By Gregory 0. Mendez, William T. Foreman, Andrew Morita, and Michael S. Majewski
}

\section{Abstract}

In 1998, the U.S. Geological Survey, in cooperation with the Sweetwater Authority, began a study to monitor water, air, and sediment at the Sweetwater and Loveland Reservoirs in San Diego County, California. The study includes regular sampling of water and air at Sweetwater Reservoir for chemical constituents, including volatile organic compounds (VOC), polynuclear aromatic hydrocarbons (PAH), pesticides, and major and trace elements.

The purpose of this study is to monitor changes in contaminant composition and concentration during the construction and operation of State Route 125. To accomplish this, the study was divided into two phases. Phase One sampling (water years 1998-2004) determined baseline conditions for the detection frequency and the concentrations of target compounds in air and water. Phase Two sampling (starting water year 2005) continues at selected monitoring sites during and after construction of State Route 125 to assess the chemical impact this roadway alignment may have on water quality in the reservoir. Water samples were collected for VOCs and pesticides at Loveland Reservoir during Phase One and will be collected during Phase Two for comparison purposes. Air samples collected to monitor changes in VOCs, PAHs, and pesticides were analyzed by adapting methods used to analyze water samples. Bed-sediment samples have been and will be collected three times during the study; at the beginning of Phase One, at the start of Phase Two, and near the end of the study.

In addition to the ongoing data collection, several special studies were initiated to assess the occurrence of specific chemicals of concern, such as trace metals, anthropogenic indicator compounds, and pharmaceuticals. This report describes the study design, and the sampling and analytical methods, and presents data from water and air samples collected during the fourth and fifth years of Phase One of the study (October 2001 to September 2003). Data collected during the first three years has been previously published.
Three types of quality-control samples were used in this study: blanks, spikes, and replicates. Blanks and spikes are used to estimate result bias, and replicates are used to estimate result variability. Additionally, surrogate compounds were added at the laboratory to samples of VOCs, PAHs, pesticides, anthropogenic indicator compounds, and pharmaceuticals to monitor sample-specific performance of the analytical method.

\section{Introduction}

The Sweetwater Authority (hereinafter referred to as the "Authority") under the guidance of its Board of Directors, operates a public drinking-water supply system for over 175,000 residential and commercial customers in Chula Vista, National City, and Bonita, California. The Sweetwater Reservoir (SWR) (fig. 1), which has a storage capacity of $34.6 \mathrm{hm}^{3}$ (cubic hectometer), is located about $15 \mathrm{~km}$ southeast of San Diego, California. The Authority also stores water at Loveland Reservoir (LLR) (fig. 1), which has a storage capacity of $31.3 \mathrm{hm}^{3}$ and is located about $30 \mathrm{~km}$ east of SWR near Alpine, California. In addition to the two reservoirs, the Authority operates three deep wells in National City and several wells near the Sweetwater River in Chula Vista. The Reynolds Desalination Facility, formerly known as the Demineralization Facility, in Chula Vista, treats brackish ground water from the wells along the Sweetwater River. Approximately 70 percent of the water the Authority provides comes from local supplies that include the Sweetwater River, the Sweetwater alluvium, and the San Diego Groundwater Formation. The remaining water is imported from the Colorado River and the Sacramento/San Joaquin River systems through pipelines and aqueducts. Local reservoir and imported waters are treated at the Robert A. Perdue Treatment Plant (hereinafter referred to as the "Perdue Treatment Plant") located at the SWR. 


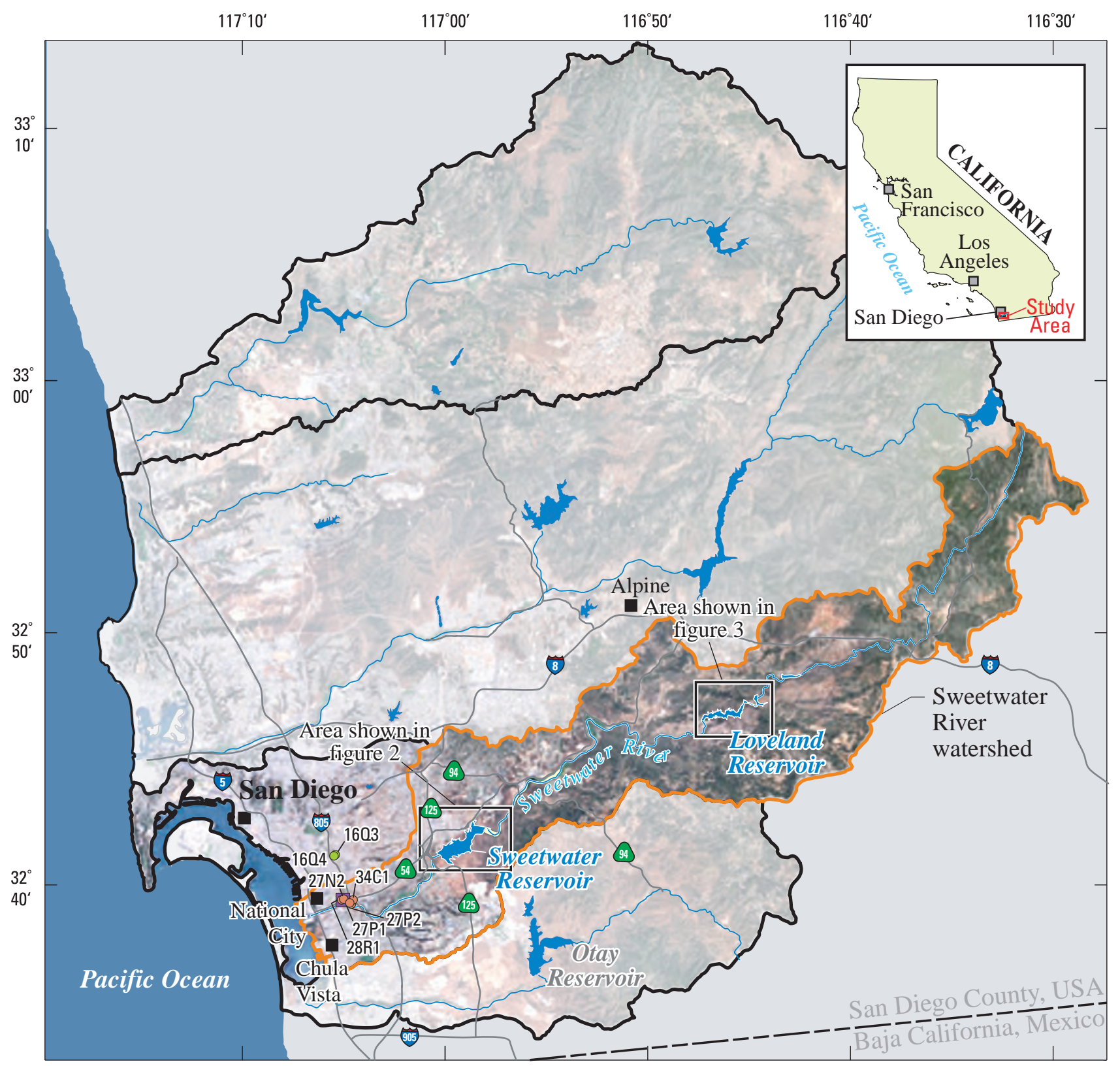

\section{EXPLANATION}

- Reynolds Desalination Facility

- Alluvial wells

- National City wells

Figure 1. The general vicinity of Sweetwater and Loveland Reservoirs and surrounding watershed, San Diego County, California.

The Sweetwater Reservoir watershed covers $466 \mathrm{~km}^{2}$ (fig.1). Although much of the land is undeveloped, the area includes agricultural land, rural residential acreage, urban and suburban residential developments, three 18-hole golf courses, mining and industrial land uses, commercial recreation, commercial business development, and two Indian reservations (both having gaming facilities), in addition to part of the Cleveland National Forest. SWR started a shore fishing program in 2005 that is restricted to an area on the south side of the reservoir. Shore fishing is allowed at Loveland Reservoir in a restricted area at the east end of the reservoir. The Authority maintains one motorized boat at each reservoir for routine water-quality sampling and regular shore patrols. 


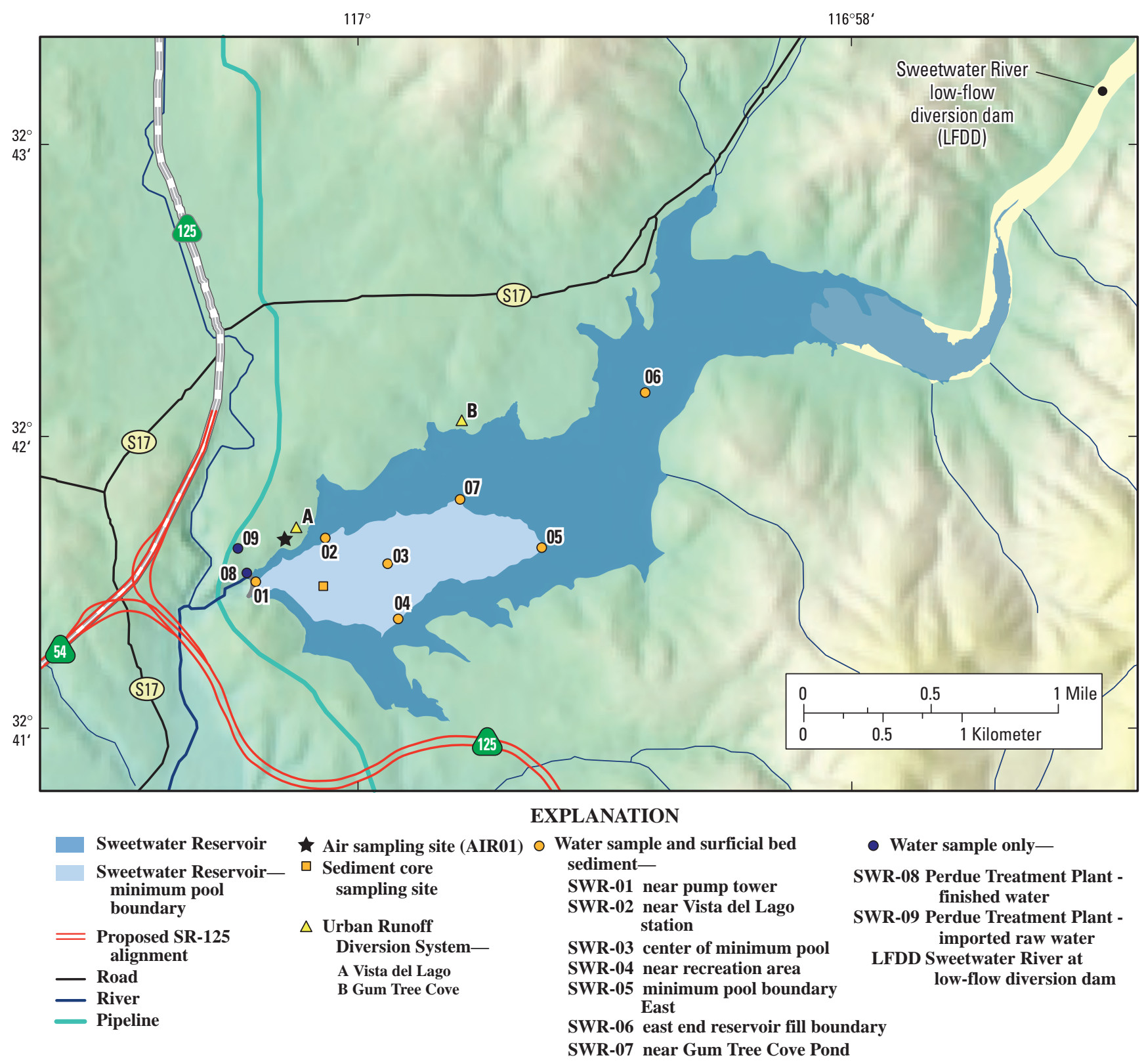

Figure 2. Sweetwater Reservoir and SR125 alignment, San Diego County, California.

The Authority is concerned about the impact that increasing growth and development in the Sweetwater Reservoir watershed will have on the quality of the drinkingwater supply and has initiated efforts to protect the watershed. These efforts include determining the amount of total organic carbon (Fram and others, 2001), identifying issues through a watershed stakeholders' outreach program, and the constructing and operating an urban runoff diversion system (URDS; fig. 2). These programs help the Authority evaluate and manage the overall environmental health of the watershed by monitoring changes that can degrade the quality of the water supply and necessitate additional water treatment as the population increases and land use intensifies. Responding to concerns about maintaining the quality of the drinking-water supply, in 1998, the Authority initiated a monitoring study in cooperation with the U.S. Geological Survey (USGS).

In addition to the increasing urbanization pressures within the watershed, the Authority is concerned about the construction and operation of the State Route (SR) 125 alignment project. In 1984, the San Diego Association of Governments added SR 125 to the Regional Transportation Plan as part of San Diego's future highway system. The SR 125 project consists of approximately $18 \mathrm{~km}$ of roadway construction/alignment that extends from SR-54 (northern terminus) to Interstate 905 (southern terminus) (fig. 1). The project plans call for the initial construction of a four-lane toll way that will be expanded to eight lanes with two additional lanes for dedicated transit purposes such as high-occupancy vehicle or light-rail operations (California Department of 
Transportation, 2001) Approximately 200,000 vehicles per day, including a high percentage (more than 10 percent) of heavy diesel trucks from both the United States and Mexico, are expected to travel SR-125. The alignment will be elevated about $30 \mathrm{~m}$ above land surface at its highest point and will bring SR 125 within about $150 \mathrm{~m}$ of the reservoir at its nearest point. Because the SWR is downwind of the proposed alignment, the Authority became concerned that toxic vehicle emissions, paved road dust, and pesticides used on the roadside might enter the reservoir by atmospheric deposition in concentrations that could effect public health and impact the cost of treating the drinking-water supply.

In 1996 the Authority commissioned a study (Ogden Environmental and Energy Services, 1997) to model the atmospheric depositional loading to the SWR. The model incorporated variety of toxic compounds from vehicular fuel combustion emissions and any attendant health risks associated with the SR 125 alignment. The predicted concentrations of select contaminants were compared with the standards set by the California Safe Drinking Water Act (California Environmental Protection Agency, 1986) and with the California and federal maximum contaminant levels (MCL) for drinking water. The model simulations indicated that drinking-water guidance levels for one or more contaminants would be exceeded by the freeway construction options. This study was repeated in 1999 reaching similar conclusions (Byard, 1999). Both the Authority and the U.S. Environmental Protection Agency Region 9 concluded that the findings in the Ogden and Byard reports warranted the implementation of a monitoring program to characterize the effects that atmospheric deposition of vehicular emissions from the operation of SR 125 may have on the quality of the drinking water stored in the SWR.

\section{Purpose and Scope}

This report presents data for water and air samples that were collected from October 2001 to September 2003 (water years 2002 and 2003) from the Sweetwater Reservoir watershed. This report is the third in a series of reports that describe the monitoring activities and present the data that will be used to assess the chemical effect of land-use changes and development in the watershed on water quality in the reservoir. Data derived from water- and air-quality samples and bedsediment samples collected during the first year's sampling (1998-1999) were presented by Majewski and others (2002). Data collected during the second and third year's sampling (1999-2001) were presented by Mendez and others (2007). Bed sediments were not sampled during the time frame covered by this report.

This study will compare analytical results collected over time from three environmental media-water, air, and bed sediment—and determine whether any measured changes in reservoir water quality are the result of atmospheric deposition of organic chemicals and metals originating from constructing and operating SR 125. In addition to providing data reports such as this one, a full analysis and assessment of the data will be written at the completion of the study.

\section{Study Design}

The study design consists of two phases: Phase One sampling started in October 1998 and continued through September 2004, the approximate start of construction for SR 125; Phase Two sampling started in October 2004, and will continue for two years after the completion of SR 125 in 2007. The objective of Phase One is to determine the occurrence and concentrations of select organic and inorganic contaminants in each of the three environmental media (water, air, and bed sediments) in order to establish baseline conditions for targeted compounds. The objective of Phase Two is to continue monitoring chemical constituents during construction and subsequent use of SR 125, to compare the analytical results to those of Phase One and thus assess the effect of SR 125 on water quality in the SWR. Two classifications of sampling were assigned: "Primary Sampling", for a common set of compounds; and, "Special Studies Sampling", to assess the occurrence of specific chemicals of concern, such as trace metals, anthropogenic indicator compounds, and pharmaceuticals. The water samples analyzed for volatile organic compounds (VOC), polycyclic aromatic hydrocarbons (PAH), and pesticides represent the "Primary Sampling."

\section{Primary Sampling}

As part of the primary sampling, each of the three environmental media in the Sweetwater Reservoir watershed was sampled and analyzed for those categories of compounds expected to accumulate in them. The categories of compounds in the three media analyzed were

- Surface water - VOCs, PAHs, and pesticides;

- Air-VOCs, PAHs, and pesticides;

- Bed sediments-PAHs, total polychlorinated biphenyls (PCB), pesticides, and major ions and trace metals.

Surface-water samples were originally collected from seven sites at SWR (sites SWR01-07, fig.2) and from two at LLR (fig. 3) as part of the primary sampling. Regularly scheduled water sampling began at these sites in 1998. Starting in 1999, samples have been collected from only three of the seven sites at SWR and one site at LLR. 


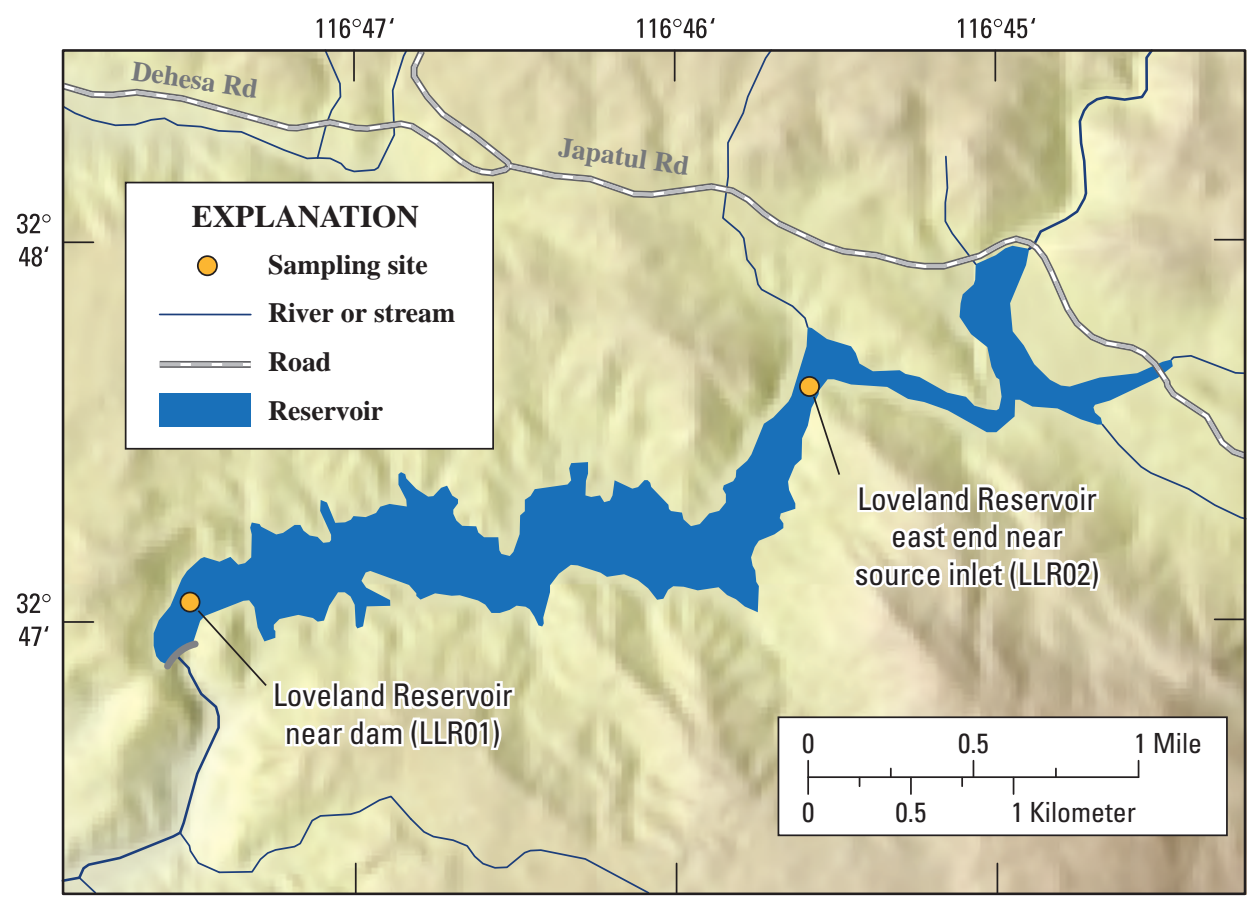

Figure 3. Sampling sites at Loveland Reservoir, San Diego County, California.

Air samples were collected from air sampling site AIR01 (fig. 2), located downwind of the proposed SR 125 route and upwind of the SWR along a transect of the predominant wind direction. The site is wired for AC power (120 volts), equipped with telephone modem access, and secured from the general public by a $2-m-h i g h$ chain-link fence. The site includes a fully instrumented meteorological station that records hourly averages of wind speed and direction, ambient air temperature and relative humidity at two heights, rainfall, barometric pressure, and atmospheric stability. Air samples were not collected at LLR because its foothill location (30 km east of SWR) was too far downwind of SR 125 to be significantly effected by any airborne contaminants originating from SR 125.

\section{Special Studies}

During the course of this study, there have been opportunities to conduct "special studies" to improve understanding of how different operational modes of the reservoir effect the quality of the water entering the treatment plant. These special studies are of limited scope and samples are collected at targeted (subset) locations. These studies provide up-to-date water-quality information on chemicals of current concern.

Because new contaminants of concern are discovered often, this current study has worked closely with scientists in the Methods Research and Development Program (MRDP) and in Analytical Services at the USGS National Water Quality Laboratory (NWQL) to help develop new analytical methods. During the time frame of this report, special studies included collecting and analyzing trace metals in selected whole and filtered water samples, and anthropogenic indicator compounds (AIC) and pharmaceutical compounds in selected filtered-water samples.

\section{Sampling and Analytical Methods}

Reservoir water samples for all analyses were collected at both reservoirs using a 1.2-L (liter), Teflon Kemmerer sampler (Wildco, Saginaw, Mo). Procedures for collecting and processing filtered-water samples to determine chemical content were based on protocols used by the USGS National Water Quality Assessment (NAWQA) Program (Shelton, 1994, 1997). Samples from the Sweetwater River at the low-flow diversion dam (LFDD) were obtained as "grab samples" collected by submerging the sample bottle, removing the cap, filling the bottle, and recapping the bottle while still submerged. The finished (treated for drinking) and imported raw-water samples were collected from spigots in the Perdue Treatment Plant. The water lines run continually and, hence, were well flushed before the sample bottles were filled. Samples collected for the analysis of VOCs and PAHs according to NWQL schedules 2020 and 1383, respectively, were not filtered. 
Samples collected for methods determining pesticide, anthropogenic indicators, and pharmaceutical compounds were filtered through 142-mm (millimeter) diameter, 0.7- $\mu \mathrm{m}$ (micrometer) nominal pore size glass-fiber filters into cleaned (baked) sample bottles (Sandstrom, 1995). All water samples were shipped on ice to the USGS NWQL. Before reservoir water samples were collected, a depth profile of dissolved oxygen, $\mathrm{pH}$, specific conductance, and temperature were measured using a multi-parameter probe. The multi-parameter probe was calibrated, and measurements were recorded, at the Perdue treatment plant before the reservoir samples were collected. The NWQL analyzed 87 VOCs by using purge and trap capillary-column gas chromatography/mass spectrometry (P\&T GC/MS) operated in full-scan mode, as described by Connor and others (1998).

Filtered-water samples were analyzed for pesticides using NWQL schedule 2001, 2002, 2003, or 2060. The filtered water sample was passed through a 0.5-gram (g) octadecylsilyl (C-18) solid-phase extraction (SPE) column. Pesticides were eluted from the column with 2 milliliters $(\mathrm{mL})$ of ethyl acetate into a culture tube containing $0.1 \mathrm{~mL}$ of a procedural internal standard solution of three perdeuterated polycyclic aromatic hydrocarbons (acenaphthene- $d 10$, phenanthrene- $d 10$, and chrysene- $d 12$ at 1 microgram per milliliter) in toluene. The extract was reduced to about $0.15 \mathrm{~mL}$ using nitrogen gas evaporation, transferred to a $\mathrm{GC}$ vial using a toluene rinse for a final extract volume of approximately $0.25 \mathrm{~mL}$, and then analyzed. For schedule 2001, 48 pesticides and degradates were analyzed using capillary-column gas chromatography with mass spectrometric detection operated in selected-ion monitoring mode (GC/MS-SIM) (Zaugg and others, 1995; Lindley and others, 1996). For schedule 2002, 72 pesticides and degradates were analyzed using capillary-column gas chromatography with mass spectrometric detection operated in selected-ion monitoring mode (GC/MS-SIM) (Sandstrom and others, 2001). For schedule 2003, 66 pesticides and degradates, including a subset of analytes in schedules 2001 and 2002, were analyzed using GC/MS-SIM methods described by Sandstrom and others (2001) and Madsen and others (2003). For schedule 2060, 66 polar pesticides and degradates were analyzed using graphitized carbon column SPE (Carbopack B; Supelco, Belefonte, Pa.) and liquid chromatography with mass spectrometric detection operated in selected-ion monitoring mode (LC/MS-SIM) as detailed by Furlong and others (2001).

\section{Air Methods}

Air samples were collected for the analysis of VOCs, PAHs, and pesticides. For this discussion, the PAHs and pesticides are referred to as semivolatile organic compounds or "SOCs." Each VOC air sample was a 24-hour composite collected every 12th day; the dates when these samples were collected was coordinated with the dates of the air sampling events at the Chula Vista location by the California Air Resources Board (CARB) Air Toxics Program. Originally, each SOC air sample was a 24-hour/7-day weekly composite collected every third week, with a two-week time span between sample collection periods. However, during the time frame of this report, each sample was collected every fourth week and the non-collection time span was increased to three weeks.

\section{Volatile Organic Compounds}

The low-volume VOC air sampling and analytical methods were developed by, and all samples were analyzed by, the research group of Dr. James Pankow at the Oregon Graduate Institute of Oregon Health and Science University. The VOC air samples were collected and analyzed by adsorption/thermal desorption GC/MS procedures as detailed by Pankow and others (1998, 2003). Ambient, gas phase, atmospheric VOC concentrations were monitored using two programmable low-volume air sampling pumps (224-PCXR8, SKC Inc., Eight Four, Pa.). One sampler pulled approximately 1.5 L of air through a glass cartridge containing $50 \mathrm{mg}$ (milligram) of Carbotrap B in series with $280 \mathrm{mg}$ of Carboxen 1000 (Supelco, Bellefonte, Pa.); these samples were analyzed for chlorofluorocarbons and other compounds with low breakthrough volumes (the VOCs, with the highest volatility). The second sampler pulled approximately $5 \mathrm{~L}$ of air through a glass cartridge containing $180 \mathrm{mg}$ of Carbotrap B in series with $70 \mathrm{mg}$ of Carboxen 1000 (Supelco, Bellefonte, Pa.); these samples were analyzed for VOC analytes with higher breakthrough volumes (VOCs with lower volatility). The method included 87 VOCs, ranging in volatility from that of dichlorodifluoromethane (CFC-12) to 1,2,3-trichlorobenzene. VOC concentrations in air are reported in parts per billion by volume (ppbv). These VOC air methods have been used in several other USGS studies that investigated the occurrence and distribution of VOCs in air (Baehr and others, 1999a,b; Bender and others, 2000).

\section{Semivolatile Organic Compounds}

The research methods for SOCs in air used in this monitoring program are similar to those described by Foreman and others (2000) for pesticides, and to USEPA methods TO-4A for pesticides (U.S. Environmental Protection Agency, 1999a) and TO-13A for PAH (Foreman and others, 1995; U.S. Environmental Protection Agency, 1999b). The air methods were developed to complement those used to analyze pesticides in water (Zaugg and others, 1995; Sandstrom and others, 2001) and PAHs/alkyl PAHs in sediment (Olson and others, 2004) The sampling and analytical methods used for the PAH and pesticide air samples have been used in several specialized studies undertaken by the USGS (Foreman and others, 1997, 2000; Majewski and others, 1998; Mendez and others, 2007). SOC concentrations are reported in nanograms per cubic meter of air $\left(\mathrm{ng} / \mathrm{m}^{3}\right)$. At this time, no air data have been entered into the USGS National Water Inventory System (NWIS) database. 
The high-volume air samples for SOCs were collected by drawing air through a 90-mm diameter glass-fiber filter (GFF; type A/E, Pall Corp., East Hills, N.Y.) and then through a cartridge containing two polyurethane foam (PUF) plugs. The GFF, containing collected atmospheric particles, was analyzed to provide the operationally-defined particulatephase concentration of an SOC. The PUF plugs collect the operationally-defined gas-phase concentration of an SOC. Before being used, the GFFs were cleaned by baking them at 450 degrees Celsius $\left({ }^{\circ} \mathrm{C}\right)$, then allowed to cool for at least 2 hours before being weighed to the nearest $0.2 \mathrm{mg}$, then wrapped in baked aluminum (Al) foil and stored in resealable polyethylene bags. At SWR, a GFF was removed from the foil with clean (methanol-rinsed) stainless-steel forceps and placed in a perfluoralkoxy fluoropolymer (Teflon-PFA) filter holder (series 90, Savillex Corp., Minnetonka, Minn.) that was modified in two ways: (1) the outer closure piece of the filter holder was removed to provide an 80-mm diameter opening that would improve air flow and expose most of the GFF surface, enabling it to collect atmospheric particles; and, (2) the holder's inner closure piece was machined to include a 3.8-cm female National Pipe Thread (NPT) to allow a direct connection to the $3.8-\mathrm{cm}$ male NPT threaded connection on the inlet of the PUF cartridge.

PUF plugs were $5 \mathrm{~cm}$ (centimeter) in diameter by $7.6 \mathrm{~cm}$ long and were prepared from open-cell foam that had an average density of $0.043 \mathrm{gm}^{-3}$ (gram per cubic meter) and contained no polybrominated diphenyl ether flame retardants (Netherland Rubber Company, Cincinnati, Ohio). PUF plugs were cleaned by first being rinsed with tap water and then sequentially extracted for at least 12 hours in a "Soxhlet" extractor apparatus with each of the following solvents: acetone; 30 percent ethyl acetate in hexane; and, dichloromethane. Residual solvent was squeezed from the PUF plugs using a potato masher, and the plugs were dried in a vacuum oven at $40^{\circ} \mathrm{C}$ for at least 48 hours before being stored in sealed 500-mL wide-mouth jars with Teflon-lined lids. At the air sampling site, primary (top) and secondary (bottom) PUF plugs were positioned in series inside a $24.2-\mathrm{cm}$ long by $3.5-\mathrm{cm}$ internal diameter Teflon-PFA cartridge (Savillex Corp.); the bottom PUF was held in place against a Teflon-PFA screen. PUF plugs were carefully inserted into the PUF cartridge using methanol-rinsed stainless-steel forceps to ensure that the PUF plugs were well fitted to the cartridge wall without creases that would allow air to migrate around the plug instead of passing through the foam.

The PUF cartridge was then connected to the GFF holder, and the GFF-PUF sampling train was positioned inside a high-volume sampler enclosure (Graesby-GMW, Village of Cleves, Ohio) comparable to that described in USEPA method TO-4A (U.S. Environmental Protection Agency, 1999a). The outlet of the PUF cartridge was connected via $0.95-\mathrm{cm}$ outer diameter Teflon tubing to a blower motor. Air samples were collected at the sampling sites by pulling ambient air through the GFF-PUF sampling train at flow rates of 28 to
$34 \mathrm{~L} / \mathrm{min}$ (liter per minute) for seven days, providing sample volumes ranging from 262 to $372 \mathrm{~m}^{3}$ (cubic meter) for samples described in this report. Sampling periods were controlled by a timer, and sample volume was calculated by multiplying the sample collection time by the air flow rate determined by using a calibrated flow meter. The time period between sample collections was typically 21 days (or more) for those samples given in this report. After the samples were collected, the GFF was removed from the holder using clean forceps, returned to the Al foil, folded in half (particle-laden side inward), and sealed in the foil and bag. PUF plugs were returned to the jars using forceps; the jars were labeled to identify the top or bottom PUF and were tightly sealed with the lids. GFF and PUF were stored at $-5^{\circ} \mathrm{C}$ (maximum), if necessary, before being shipped overnight on ice packs to the USGS NWQL.

One field-blank sample to be analyzed for SOCs was collected to examine any possible contamination resulting from field handling of the GFF-PUF sampling components and from post-collection sample processing steps. The blank was obtained by loading one clean GFF and two clean PUF plugs into the sampling train at the field laboratory, taking the train out to the site to install and uninstall the train, returning the train to the field lab, and then unloading the GFF and PUF plugs for storage and shipping. No air was drawn through the sampling train while collecting the blank.

At the NWQL, air sample components were stored at $-5^{\circ} \mathrm{C}$ (maximum) until analysis. GFFs were desiccated for 24 hours and weighed to the nearest $0.2 \mathrm{mg}$ to determine particle weight. This weight was divided by the sample's air volume to determine the total suspended particle (TSP) concentration in micrograms of particles per cubic meter of air $\left(\mu \mathrm{g} / \mathrm{m}^{3}\right)$.

Samples collected during two periods of time were extracted using two different procedures. All samples collected through January 2002 were extracted using traditional solvent extraction procedures. Each GFF was placed in a 500-mL, flat bottom flask, and each PUF plug was placed in a separate Soxhlet apparatus. GFF or PUF plugs were fortified with surrogate compounds (see Quality Control section) and extracted with 100-mL (GFF) or 300-mL (PUF) of 30-percent ethyl acetate in hexane for at least 12 hours. These samples are identified as extracted by Soxhlet. Samples collected from February 2002 through September 2003 were extracted using a procedure referred to as pressurized-solvent extraction, which relies primarily on the elevated temperature of a pressurized solvent to achieve rapid extraction (U.S. Environmental Protection Agency, 1999; Alexandrou and others, 2001). Each GFF was placed into an 11-mL stainless-steel cell, and top and bottom PUF plugs were put into separate $33-\mathrm{mL}$ cells before the compounds were extracted. The GFF or PUFs were fortified with surrogate compounds (see Quality Control section) and extracted using an accelerated solvent extractor, ASE 200 (Dionex Corp., Salt Lake City, Utah), using two 15 -min static cycles at $120^{\circ} \mathrm{C}(\mathrm{GFF})$ or $100^{\circ} \mathrm{C}$ (PUF) under pressurized conditions (10,342 kilopascals). (Note: Top and 
bottom PUF plugs used for samples collected during this reporting period were extracted and analyzed separately to determine PUF collection efficiencies for gas-phase SOCs.)

All extracts were dried with sodium sulfate and reduced in volume using Kuderna-Danish distillation and nitrogen gas evaporation to $1 \mathrm{~mL}$ (or $2 \mathrm{~mL}$ for samples collected after Dec. 2002). For samples collected before January 2003, the extract was introduced to a $0.5-\mathrm{g}$ (gram) $\mathrm{C}-18 \mathrm{SPE}$ column positioned above a 1-g Florisil SPE column (Biotage, Charlottesville, Va.; columns were pre-rinsed with acetone, dichloromethane, and ethyl acetate). Analytes were eluted with 6-mL of ethyl acetate, which was reduced to about $0.3 \mathrm{~mL}$ by nitrogen gas evaporation, and transferred with a $0.15-\mathrm{mL}$ ethyl acetate rinse to a $\mathrm{GC}$ vial containing $500 \mathrm{ng}$ (nanogram) of 1,4-dichlorobenzene and five perdeuterated polycyclic aromatic hydrocarbons (naphthalene- $d 8$, acenaphthene- $d 10$, phenanthrene- $d 10$, chrysene- $d 12$, and perylene- $d 12$ ) in ethyl acetate, by internal injection standards. Air extracts were analyzed by GC/MS-SIM for PAHs and alkyl PAHs using instrumental procedures comparable to those described by Olson and others (2004). This analysis is referred to as the "AirPAH" method in this report. Concentrations for any detected alkylated-PAH were estimated (E) because alkylatedPAH standards were not available when calibration standards were prepared. The alkylated-PAHs were quantified using the calibration curves derived from appropriate parent PAHs (Olson and others, 2004).

Extracts of air samples collected until February 2002 also were analyzed for many of the same pesticides and degradates included in the water methods (NWQL schedules 2001 and 2002) by using the GC/MS-SIM instrumental procedures described by Zaugg and others (1995) and Sandstrom and others (2001). These methods are referred to as "Airpest001" and "Airpest002", respectively, in this report. Samples collected from February 2002 through September 2003 were analyzed by GC/MS-SIM (comparable to NWQL water schedule 2003) for the 66 compounds that are a subset of the high and moderate-use pesticides and degradates determined using methods described by Zaugg and others (1995), Sandstrom and others (2001), and Madsen and others (2003). This pesticide analysis is referred to as "Airpest003" method in this report. Some analytes included in the water methods (NWQL schedules 2001, 2002, and 2003) were not amenable to the Airpest001, Airpest002, and Airpest003 methods because the compounds were inadequately collected by PUF or recovered during the SPE cleanup step; therefore, these compounds were not reported for the air samples. Also, three endosulfan compounds were included in the Airpest001 method, although they were not determined by NWQL schedule 2001 for the water samples.

Starting in January 2003, the air-sample-extract cleanup procedure was modified to improve recoveries of certain pesticides and degradates that were inadequately recovered during the C18/Florisil cleanup. After samples were extracted, extracts from these samples were reduced to about $2 \mathrm{~mL}$ and quantitatively (by weight) split approximately in half before column cleanup; one portion (PAH fraction) was processed through the C18/Florisil SPE procedure and analyzed for PAHs and alkyl PAHs by GC/MS- SIM or full scan (top PUF plugs only) mode as described above except that the final extract volume before GC/MS analysis was $0.25 \mathrm{~mL}$ instead of $0.5 \mathrm{~mL}$. The remaining portion (pesticide fraction) was added to a 0.5-g graphitized carbon SPE column (CarboPrep 90, Restek Corp., Bellefonte, Pa.; pre-rinsed with acetone, dichloromethane, and ethyl acetate) and the analytes were eluted with $13 \mathrm{~mL}$ of a 50 percent dichloromethane in ethyl acetate solution. The effluent was reduced using microKuderna-Danish distillation to about $1 \mathrm{~mL}$. A procedural internal standard solution of three perdeuterated polycyclic aromatic hydrocarbons (acenaphthene- $d 10$, phenanthrene- $d 10$, and chrysene-d12) in toluene was added to the extract before final solvent reduction to about $0.15 \mathrm{~mL}$ using nitrogen gas evaporation. The extract was transferred to a $\mathrm{GC}$ vial with a toluene rinse for an approximate $0.25-\mathrm{mL}$ final volume for analysis by GC/MS-SIM instrumental procedures using Airpest003 method.

\section{Special Studies Methods}

Data from three special studies collected during the 2-year time frame covered by this report were analyzed. The first special study examined concentrations of trace metals in water samples collected in the reservoir. By comparing concentrations in the reservoir to airborne particle concentrations, we can infer what fraction may have come from the alignment of SR125 with a new section of toll road. A variety of trace metals are emitted from auto exhaust, tire and brake wear, and road dust. Samples were collected at SWR01 (fig. 2) and analyzed as either unfiltered (whole-water) or filtered (operationally-defined dissolved phase) water types. Trace metals in unfiltered-water samples were analyzed using inductively coupled plasma-optical emission spectrometry and inductively coupled plasma-mass spectrometry using methods described by Garbarino and Struzeski (1998). Dissolved trace metals in filtered-water samplers were analyzed by inductively coupled plasma-mass spectrometry using methods described in Faires (1993).

The second special study comprised sampling and analysis for 62 anthropogenic indicator compounds (AIC), some of which are typically associated with wastewater effluent. Water samples collected for AIC were filtered through $0.7-\mu \mathrm{m}$ (micrometer) nominal pore size GFFs into new glass sample bottles (Sandstrom, 1995). Approximately 1-L filtered water samples were fortified with four surrogate compounds (see Quality Control section) and passed through a 1-g Oasis HLB SPE column (Waters Corp., Milford, Mass.). Eluted analytes (including some classified as endocrine disrupting compounds) were determined by GC/MS operated in full-scan mode. The USGS NWQL developed an analytical method that determines representative compounds from various chemical classes to monitor unregulated and 
regulated contaminants typically associated with wastewater effluent (Zaugg and others, 2002), including some endocrine disrupting compounds.

The third (last) special study comprised sampling and analysis for 14 pharmaceutical compounds. These compounds were isolated from approximately 1-L filtered-water samples (fortified with ethylnicotinate- $d 4$ as surrogate) using a 1-g Oasis HLB SPE column. Eluted analytes were determined by liquid chromatography with electrospray mass spectrometric detection operated in selected-ion monitoring mode as detailed by Cahill and others (2004).

\section{Sampling Strategy and Data}

The following sections present the strategies used to collect water and air samples, as well as the corresponding data tables. Some data tables show more than one laboratory reporting level for a compound or an element in different samples. This occurs, in part, because reporting levels are updated annually, as necessary, as a component of the NWQL's yearly assessment of long-term method detection levels and laboratory reporting levels (Childress and others, 1999). Reporting levels also vary because of differences in sample volumes or the presence of interferences. Some concentrations are reported as estimated (E). In these cases, mass spectral results indicated that the sample contained the compound, but the concentration was less certain because it was below the lowest calibration standard or the laboratory reporting level.

\section{Water Sampling Strategy and Data}

Water samples were collected at three sites in SWR (SWR01, SWR03, and SWR06; fig 2), two sites at the Perdue Treatment Plant (SWR08 and SWR09; fig.2), one site on the Sweetwater River above SWR (LFDD; fig.2), and one site at Loveland Reservoir (LLR01) (fig. 3). The analyses of the water samples, except pharmaceutical data, have been entered into the USGS National Water Information System (NWIS) database. All sites within SWR, except site SWR06, are within the reservoir's minimum pool boundary (fig. 2) to ensure that water will be available for sampling throughout the year. SWR06 is in the northeastern third of the reservoir in very shallow water. When the water level falls, the water depth at SWR06 decreases, and sometimes the bed sediments are completely exposed. Whenever this has occurred, the sampling site was moved to a nearby location where the water depth was about one meter deep. Sampling sites at both SWR and LLR are marked with stationary buoys anchored to the bottom.

Three additional water-sampling sites (SWR08, SWR09, and LFDD; table 1, fig. 2) were established outside of the reservoir boundaries. SWR08 monitors the quality of the finished water as it leaves the treatment plant for distribution to customers. SWR09 monitors the quality of the imported water before it enters the treatment plant. Sweetwater River at the Low-Flow Diversion Dam (LFDD) monitors the quality of the water entering SWR from the watershed. During low flows, the water from LFDD is diverted into the Urban Runoff Diversion System (URDS) ponds. Local urban runoff and the first flush in the Sweetwater River are diverted into the URDS ponds to prevent contaminated water from entering the SWR. A first flush refers to the initial runoff from a storm or reservoir release after a dry period. All site identification numbers, other identifiers, and sampling site names are listed in table 1.

Imported water is usually pumped directly into the treatment plant. Occasionally imported water is instead pumped directly into SWR to augment the local supply. When imported water is pumped directly into the reservoir, it significantly increases the water level; volume increases can be tens of thousands of acre-feet. Imported water is never used solely to maintain the minimum pool level.

Baseline water sampling at both SWR and LLR began in September 1998 and continued at 2-month intervals through September 1999. This bimonthly sampling allowed monitoring of the effects of various operational modes of the reservoirs, such as recharge or drawdown events that significantly changed the water level in the reservoirs. It also showed the spatial variability in chemical occurrence and concentration in each reservoir. From October 1999 to December 2002, the sampling frequency was reduced to once every third month (quarterly). Beginning in December 2002, the sampling frequency was increased to every other month (bimonthly) in anticipation of the beginning of the construction of SR125.

Beginning in October 1999, the number of sampling sites at SWR was reduced from seven to three, and the number of sampling sites at LLR was reduced to one. The Perdue Treatment Plant and Sweetwater River site locations were not changed. The number of sampling sites in the reservoir was reduced to focus the sampling efforts at "indicator" sitesthose sites believed to provide the most relevant information without compromising the scientific integrity of the project. These indicator sites were

- SWR01-located near the dam and the intake for reservoir water to the treatment plant, used to monitor the quality of water entering the treatment plant.

- SWR03-located near the center of the minimum pool, used to monitor the quality of water at the center of the reservoir.

- SWR06-located near the east end of the reservoir, used to monitor the quality of water entering the reservoir from the Sweetwater River.

- LLR01-located near the dam, used to monitor the quality of the reserve storage water. 
Table 1. Site identification numbers, site numbers, and corresponding site names, Sweetwater and Loveland Reservoirs, San Diego County, California.

[See figure 2 for site locations]

\begin{tabular}{cll}
\hline $\begin{array}{c}\text { Site identification } \\
\text { No. }\end{array}$ & $\begin{array}{c}\text { Other } \\
\text { identifiers }\end{array}$ & \multicolumn{1}{c}{ Site name } \\
\hline 324130117002501 & SWR01 & Sweetwater Reservoir near pump tower \\
324139117000801 & SWR02 & Sweetwater Reservoir near Vista del Lago station \\
324131117000101 & SWR03 & Sweetwater Reservoir center minimum pool \\
324126116595701 & SWR04 & Sweetwater Reservoir near recreation area \\
324137116592401 & SWR05 & Sweetwater Reservoir minimum pool boundary East \\
324209116585001 & SWR06 & Sweetwater Reservoir east end reservoir fill boundary \\
324147116593501 & SWR07 & Sweetwater Reservoir near Gum Tree Cove Pond \\
324132117002701 & SWR08 & Perdue Treatment Plant-finished water at Sweetwater Reservoir \\
324137117002901 & SWR09 & Perdue Treatment Plant-imported raw water at Sweetwater Reservoir \\
324311116565901 & LLDD & Sweetwater River at low flow diversion above Sweetwater Reservoir \\
$324703116473101^{*}$ & LLR01 & Loveland Reservoir near dam \\
$324737116453501^{*}$ & LLR02 & Loveland Reservoir east end near source inlet \\
324141117001601 & AIR01 & Sweetwater Reservoir air sampling site \\
\hline
\end{tabular}

"Loveland Reservoir sites on figure 3.

Before any reservoir water was sampled, depth profiles of dissolved oxygen, $\mathrm{pH}$, specific conductance, and temperature were measured at $1-\mathrm{m}$ intervals from the surface to the bottom at each sampling location. These measurements are given in table 2. (See back of report for this and remaining tables.) At LLR, the depth-profile below $10 \mathrm{~m}$ was measured at 2-m intervals because the depth of LLR is much greater than that of SWR. If the temperature profile indicated a thermocline (thermal stratification), two sets of water samples were collected at the site: one at midepilimnion and one at midhypolimnion. The epilimnion can be defined as the layer in a lake extending from the surface to a depth where photosynthesis ends or where the slope of the temperature gradient changes. The hypolimnion can be defined as the poorly illuminated lower region of a stratified lake. The temperature of the hypolimnion is nearly uniform and oxygen is depleted. This stratum of water is characterized by decay rather than by the production of organic matter. When no thermocline was evident, only one sample set was collected at a point midway between the water surface and the reservoir bottom.

VOCs analyzed in whole-water samples (along with their applicable LRLs) are given in table 3A, and the compounds detected are given in table 3B. PAH compounds analyzed in whole-water samples are given in table $4 \mathrm{~A}$, and the compounds detected are given in table 4B. Concentrations of MTBE, benzene, and toluene in air samples at the air sampling site (fig. 2) from 1998 to 2003 are shown in figure 4A and can be compared with concentrations of these compounds in water. Concentrations of MTBE, benzene, and toluene in water samples at SWR01 and SWR03 from 1998 to 2003 are shown in figures 4B, 4C, and $\underline{4 \mathrm{D}}$, respectively. To avoid falsely implying trends in the plots due to variations in laboratory detection limits, all nondetections were plotted at a very low concentration $(0.003 \mu \mathrm{g} / \mathrm{L})$ for a normalized appearance. This normalized concentration was chosen so all of the detections were above this value.

A list of pesticide compounds analyzed for in filteredwater samples using NWQL schedule 2001 (and their applicable LRLs) are given in table 5A; those from schedule 2002 are given in table 6A; those from schedule 2003 are given in table 7A; and those from schedule 2060 are given in table 8A. The concentrations of the analytes detected are presented in the following tables according to NWQL schedule: schedule 2001 in table 5B; schedule 2002 in table 6B; schedule 2003 in table 7B; and schedule 2060 in table 8B. Concentrations of simazine at SWR01 and in finished and imported waters are shown in figure 5. To avoid falsely implying trends in figure 5 due to variations in laboratory detection limits, all nondetections were plotted at a very low concentration $(0.003 \mu \mathrm{g} / \mathrm{L})$ for a normalized appearance. This normalized concentration was chosen so all of the detections were above this value.

\section{Air Sampling Strategy and Data}

Air data were collected to determine the occurrence, temporal patterns, and ambient concentration levels of selected airborne organic compounds (VOCs, PAHs, and pesticides). Site AIR01 was installed downwind of the proposed SR 125 routes and upwind of SWR (fig. 2) along the predominant wind direction. This air sampling station was established 


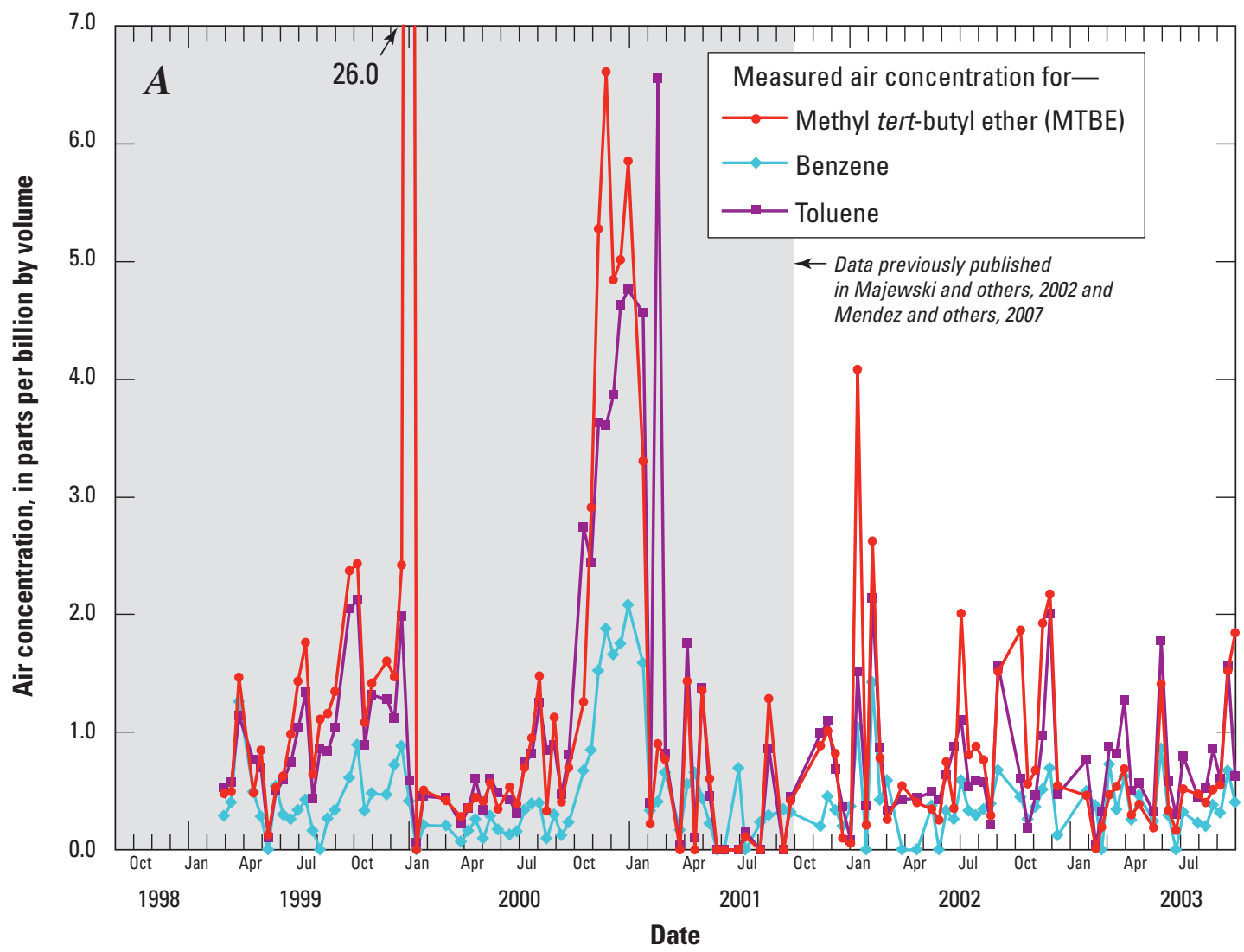

Figure 4. (A) Concentrations in air of methyl-tert-butyl ether (MTBE), benzene, and toluene at the air sampling site, and concentrations in air and in water of $(B)$ methyl-tert-butyl ether (MTBE), $(C)$ benzene, and $(D)$ toluene at Sweetwater Reservoir (SWR) near the pump tower (SWR01), and SWR center of minimum pool (SWR03), San Diego County, California.

following the guidelines outlined by the National Atmospheric Deposition Program (Bigelow, 1984), with additional guidance from the South Coast Air Pollution Control Board (William Brick, oral commun., 1999). The first VOC air sample was collected on March 23, 1999. The first PAH and pesticide air samples were collected on May 11, 1999.

The VOC air method was used to analyze 87 compounds. The results of the VOC air analyses are given in tables $9 \mathrm{~A}$ and 9B. All VOCs results for the air samples were blank corrected at the lab before the data were released. Blanks were corrected on the basis of the results of 50 to 60 travel blanks from previous and current sampling events. The upper threshold of the blank contamination for each analyte was calculated as the average (mean) plus three times the standard deviation observed in the travel blanks. Concentrations below this threshold were reported as not detected. If the concentration in a sample was higher than this threshold, then the threshold was subtracted from the sample concentration to provide the blank-corrected concentration.

Individual PAH compounds and alkylated PAH homologs determined by the AirPAH method, and estimated reporting levels, are given in table 10A. Concentrations of PAH and alkyl PAH detected in air samples are given in table 10B. Also shown in table 10B are sampled air volumes, the total suspended particulate (TSP) concentrations for the samples, how each sample component was extracted (Soxhlet extraction or pressurized solvent extraction, PSE), whether the extract was split before SPE cleanup, and whether the reported concentrations were determined by GC/MS operated in SIM or full-scan mode (typically, only the top PUF for this reporting period).

Pesticide compounds analyzed in air samples by the Airpest001 method are given in table 11A, by the Airpest002 method in table 12A, and by the Airpest003 method in table 13A. Concentrations of the six parent pesticides (benfluralin, chlorpyrifos, dacthal, diazinon, malathion, and trifluralin) and a $p, p$ '-DDT degradate, $p, p$-DDE, that were detected in the air samples using the Airpest001 method are given in table 11B. Neither the parent $p, p$-DDT nor a second degradate, $p, p$-DDD, was determined by any of the air analysis methods used. Concentrations of the parent pyrethroid insecticide bifenthrin, the malathion degradate malaoxon, and the carbaryl or napropamide (less likely) degradate 1,4-naphthoquinone were detected in air samples by the Airpest002 method (table 12B). The degradates chlorpyrifos oxon and diazoxon initially determined by the Airpest002-method were not recovered during the Florisil SPE cleanup step (see Quality Control section and Mendez 

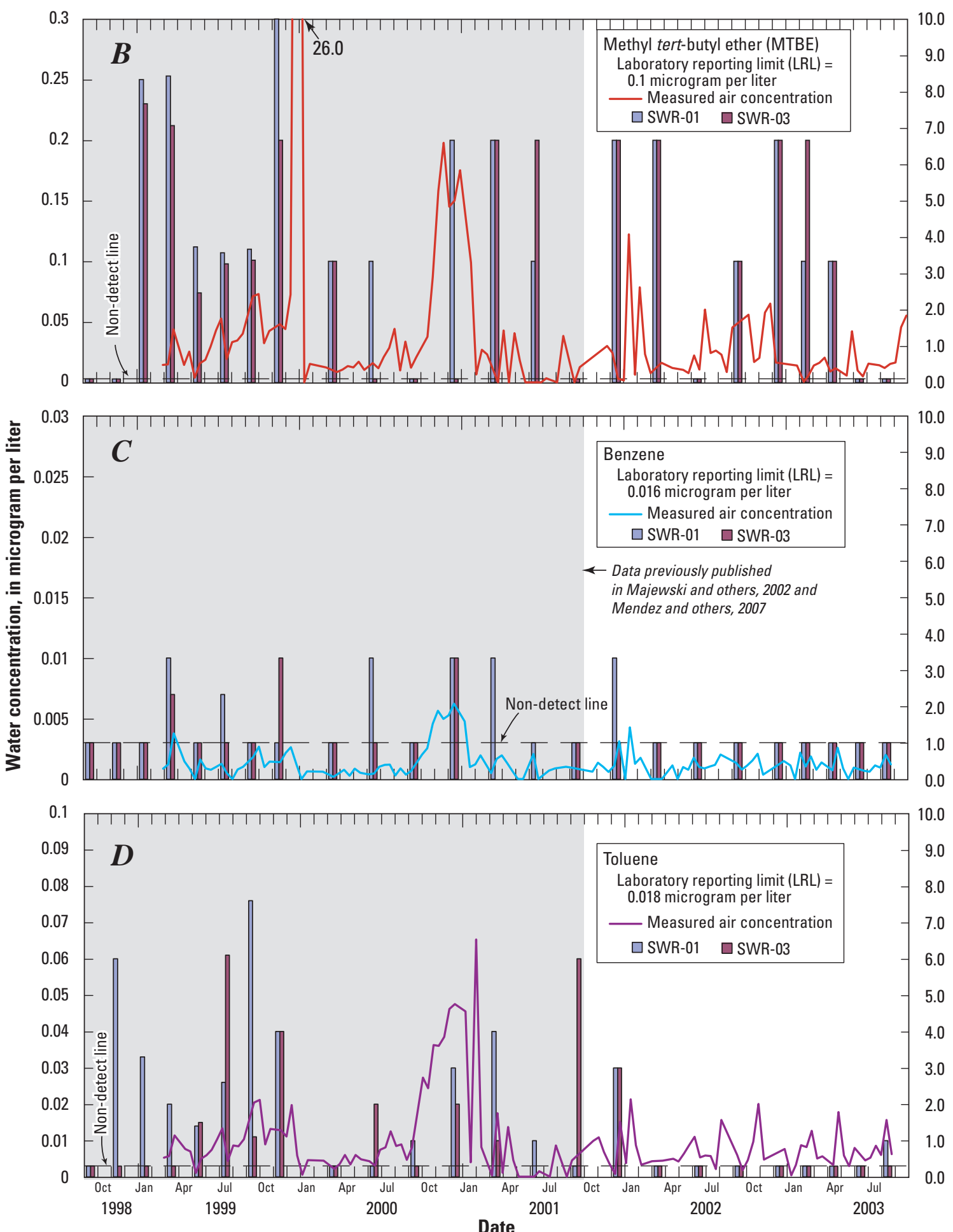

Figure 4. Continued.

and others, 2006). Concentrations of 11 parent pesticides, plus degradates diazoxon, malaoxon, deethylatrazine (from atrazine or propazine), and 4-chloro-2-methylphenol that were detected in air samples using the Airpest003 method are given in table 13B. Degradates of MCPA, MCPB, mecoprop and their salts and esters (these parent acid herbicides) were not determined by the Airpest003 method. Although the Airpest003 method includes many analytes determined by the other two air methods (11A and 12A), it does not include $p, p$ DDE, bifenthrin, and 1,4-naphthoquinone. 


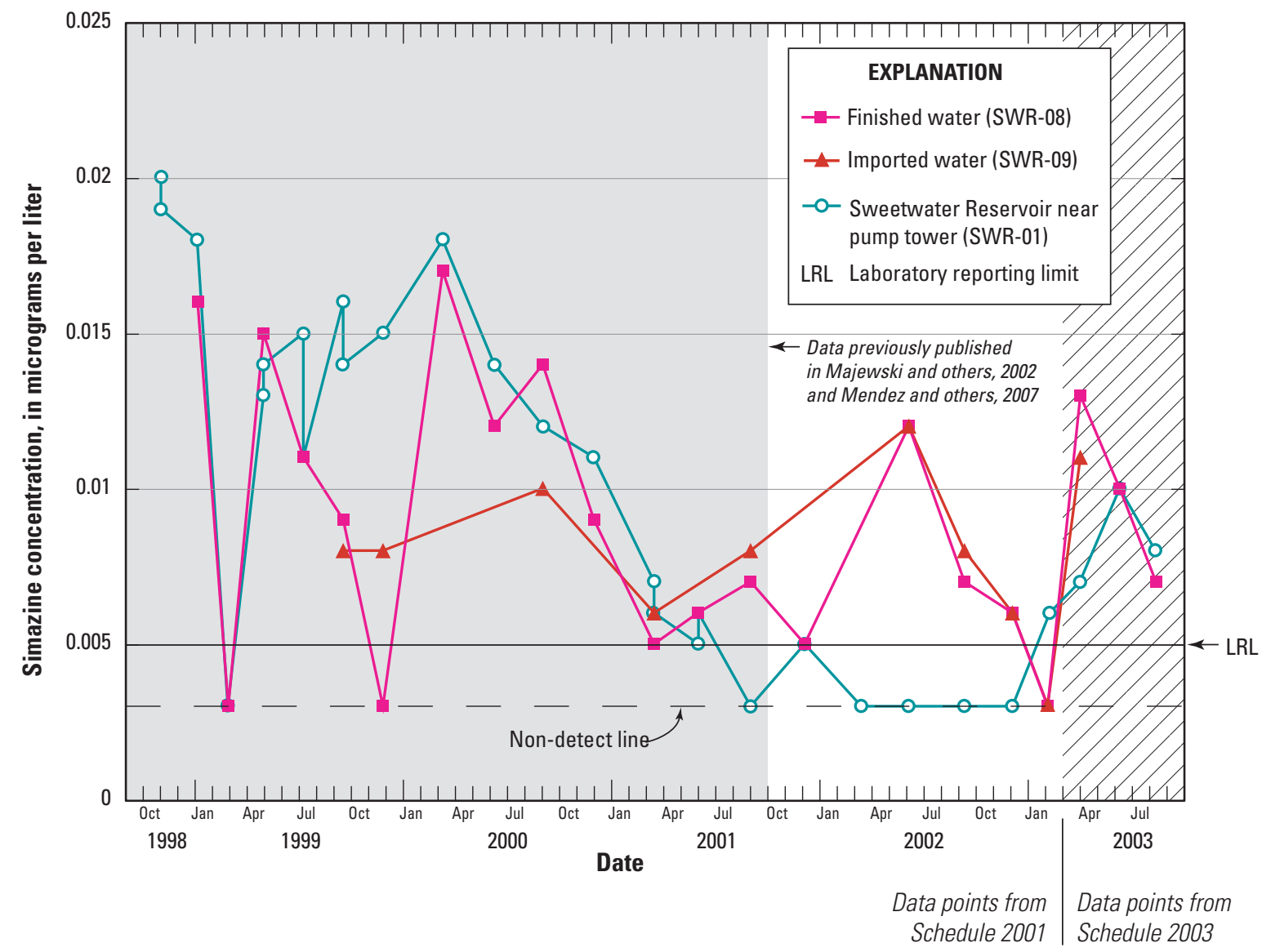

Figure 5. Concentrations of simazine in finished water at site SWR08, in imported water at site SWR09, and near the pump tower (site SWR01) at Sweetwater Reservoir, San Diego County, California.

\section{Special Studies Strategy and Data}

Three special studies were conducted during the time frame covered by this report. The first special study analyzed trace metals in filtered and unfiltered (whole) water samples collected at SWR01 (fig. 2). Whole-water samples were collected in August 2003 (table 14A). Filtered-water samples were collected from February 2003 through August 2003 (table 14B). The second special study determined a variety of anthropogenic indicator compounds (AICs; table 15A), some of which are typically associated with wastewater effluent. Concentrations of AICs detected in filtered-water samples at sites SWR01, SWR08, SWR09, and LFDD (fig. 2) are given in table 15B. The third special study analyzed pharmaceutical compounds in filtered-water samples collected at sites SWR01, SWR08, SWR09, and LFDD (fig. 2). The compounds analyzed and their detections are listed in table 16.

\section{Quality Control}

Three types of quality-control samples were used in this study: blanks, spikes, and replicates. Blanks and spikes are used to estimate result bias, and replicates are used to estimate result variability. In addition, surrogate compounds were added to VOC, PAH, pesticide, AIC, and pharmaceutical samples at the laboratory to monitor sample-specific performance of the analytical method.

Blanks should be free of the analytes of interest, and can be prepared in the laboratory or field. Blank samples are analyzed to test for bias that could result from contamination of environmental samples by the analytes of interest or by interference during any stage of sample collection, processing, and analysis. Blanks for water samples are collected by processing USGS laboratory certified reagent water known to be free of the analytes of interest through any part of the sampling and processing stage. A field blank is collected at an environmental sampling location by passing blank water through all of the field-cleaned sampling equipment. Field blanks are used to verify that no stages of sample collection and processing have introduced contamination. Equipment blanks are collected annually and used to verify that sample collection and processing equipment are not introducing contamination. Source solution blanks are collected to demonstrate that the laboratory-certified reagent water is free of the analytes of interest. Travel (trip) blanks are used to identify contamination that may occur during sample 
transport and analysis rather than as a result of sample collection and processing. "Lot blanks" are analyzed at the laboratory for a subset of the air VOC cartridges sent to be used for environmental samples to test the matrix used during manufacturing of the cartridges. Laboratory blanks are used to verify that the laboratory sample processing steps have not introduced contamination.

A spike is prepared by fortifying reagent water or the airsampling media (GFFs or PUFs) with a known concentration of selected analytes. Spiked samples are used to measure bias in analyte recovery. Matrix spikes also can be used to test the effects of various matrices on the recovery of specific compounds, including matrix-induced analyte degradation. All spiking was done in the laboratory; no field-spiked samples were analyzed during this study period.

Replicate samples are collected and processed identically to the environmental sample and used to measure the variability during sample processing and analyses. Replicate air samples were not collected during this reporting period.

\section{Water Quality-Control Samples}

One field quality-control sample type was collected during every reservoir sampling trip. The type of qualitycontrol sample collected and the sampling location were chosen randomly. Blank and replicate quality-control data for VOCs in water samples are given in table 17. The blank and replicate quality-control data for pesticides in water samples processed by schedules 2001 and 2060 are given in tables 18 and 19 , respectively.

The quality of analytical results was monitored by adding surrogate compounds to each sample before it was processed for analysis. These surrogate compounds were added at the NWQL to monitor sample preparation and analysis. For VOCs, surrogates 1,2-dichloroethane-d4, 1,4-bromofluorobenzene, and toluene- $d 8$, were added to each VOC sample. The results, in percent recovery, for VOC surrogates in water are given in table 3B. Surrogates 2-fluorophenol, 2-fluorobiphenyl, nitrobenzene- $d 5$, phenol$d 5$, terphenyl- $d 14$, and 2,4,6-tribromophenol were added to each PAH sample. The results, in percent recovery, for PAH surrogates in water are given in table 4B. For pesticides in water that were analyzed using schedules 2001, 2002, and 2003 , diazinon- $d 10$ and alpha-HCH- $d 6$ were added to each sample (tables 5B, 6B, and $\underline{7 \mathrm{~B}}$, respectively). For pesticides in water that were analyzed using schedule 2060, surrogates 2,4,5-T, barban, and caffeine- C13 were added to each sample (table 8B).

\section{Air Quality-Control Samples}

Several replicate VOC air samples were collected for low breakthrough and high breakthrough volumes; the results are given in tables 20A and 20B, respectively. Each VOC air sample was analyzed along with an associated laboratory spike, travel blank, lot blank, and laboratory blank. For chlorofluorocarbons and compounds with a low breakthrough volume, the laboratory spike, travel blank, lot blank, and laboratory blank data are in tables $21 \mathrm{~A}, 21 \mathrm{~B}, 21 \mathrm{C}$ and $21 \mathrm{D}$, respectively. For VOC compounds with a high breakthrough volume, the laboratory spike, travel blank, lot blank, and laboratory blank data are in tables 22A, 22B, 22C and 22D, respectively.

Each air sample component (GFF and top and bottom PUF plugs) was fortified with surrogate compounds at the NWQL to monitor instrument performance during sample analysis. Recovery data for surrogate compounds nitrobenzene- $d 5$, 2-fluorobiphenyl, and terphenyl- $d 14$ used to monitor performance for the PAH analysis are given in table 10 . Recovery data for surrogate compounds diazinon$d 10$ and alpha-HCH-d6 used to monitor performance for the pesticides analyses by Airpest001, Airpest002, or Airpest003 methods are given in tables $11 \mathrm{~B}, 12 \mathrm{~B}$, and $13 \mathrm{~B}$, respectively.

The air methods are similar to the water methods for assessing the efficiency of the analytical method. Groups of SOC or VOC air samples have an associated laboratory blank and laboratory spike analyzed along with the environmental samples. The associated laboratory blank and laboratory spike quality-control data for the PAHs in air samples are given in tables 23A and 23B , respectively. One field PAH blank sample for all three components was collected during this reporting period for compounds listed in table 23A. No PAH compounds were detected in this blank. To minimize risk of sampler contamination, no SOC field spike air samples were collected in this study. Instead, separate analyses of top and bottom PUF plugs were used to monitor sample-specific collection efficiency. No replicate air samples were collected.

The laboratory blank and laboratory spike data for method Airpest001 type pesticides in air are given in

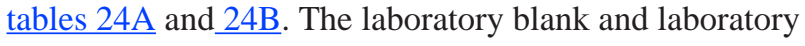
spike data for method Airpest002 pesticides in air are given in tables 25A and 25B. The laboratory blank and laboratory spike data method Airpest003 pesticides in air are given in tables 26A and $26 \mathrm{~B}$.

\section{Special Studies Quality-Control Sampling}

During the time frame covered by this report, no quality-control field samples were collected for the three special studies. Recovery data for surrogate compound Ethylnicotinate- $d 4$ that was added to each pharmaceutical sample are given in table 16. Laboratory blank and spike data for pharmaceutical compounds are listed in table 27A and $\underline{27 \mathrm{~B}}$, respectively. 


\section{Summary}

The primary purpose of this study is to monitor changes in composition and concentration of compounds in the air and water before, during, and after the construction and operation of a major thoroughfare being built upwind of the Sweetwater Reservoir. To accomplish this, the study was divided into two phases. Phase One sampling was designed to establish baseline conditions for target compounds (primary sampling) in terms of detection frequency and concentration in air and water. Phase Two sampling is planned to continue during and after construction of the roadway (SR 125) to assess the chemical impact this alignment project may have on the water quality in the reservoir. In a project of this size, many compounds are analyzed to help water agencies better understand the effects of urbanization on drinking-water reservoirs. In addition to the ongoing data collection, several special studies were initiated to assess the occurrence of specific chemicals of concern, such as trace metals, anthropogenic indicators, and pharmaceutical compounds.

Before any reservoir water was sampled, depth profiles of temperature, $\mathrm{pH}$, dissolved oxygen, and specific conductance were recorded at each reservoir sampling location (table 2). VOCs analyzed in water samples and their results are in tables $3 \mathrm{~A}$ and $\underline{3 \mathrm{~B}}$, respectively. PAHs analyzed in water samples, and their results, are in tables $4 \mathrm{~A}$ and $\underline{4 \mathrm{~B}}$, respectively. Pesticide compounds analyzed in water by National Water Quality Laboratory (NWQL) schedules are in tables $5 \mathrm{~A}, \underline{6 \mathrm{~A}}, \underline{7 \mathrm{~A}}$, and $\underline{8 \mathrm{~A}}$. Pesticide compounds detected by NAWQL schedules are in tables 5B, 6B, $\underline{7 B}$, and $\underline{8 B}$. Results of VOCs analyzed in air are in tables 9A and 9B. PAH compounds analyzed in air samples and their results are in table $10 \mathrm{~A}$ and $\underline{10 \mathrm{~B}}$, respectively. Pesticide compounds analyzed in air using three different National Water Quality Laboratory methods are in tables 11A,$\underline{12 \mathrm{~A}}$, and $\underline{13 \mathrm{~A}}$. Results for pesticide methods analyzed in air are in tables 11B,$\underline{12 B}$, and $13 \mathrm{~B}$. Data from the three special studies in water for trace metals, anthropogenic indicators, and pharmaceutical compounds are in tables 14, 15, and $\underline{16}$. Quality-control data for VOCs in water samples are in table 17. Quality-control data for pesticides in water sampling are in tables 18 and 19. Replicate data for VOCs in air samples are in tables $\underline{20 \mathrm{~A}}$ and $\underline{20 \mathrm{~B}}$. Quality-control travel-blank, spike, lot-blank, and laboratory-blank data for VOC in air samples are in tables 21A-D and 22A-D, respectively. Quality-control blank and spike data for PAH in air samples are in tables 23A and 23B. Quality-control blank and spike data for pesticide in air samples are in tables 24A-B, 25A-B, and 26A-B. Qualitycontrol blank and spike data for pharmaceutical compounds in water samples are in tables 27A and $\underline{27 \mathrm{~B}}$.

\section{References Cited}

Alexandrou, N., Smith, M., Park, R., Lumb, K., and Brice, K., 2001, The extraction of polycyclic aromatic hydrocarbons from atmospheric particulate matter samples by Accelerated solvent extraction (ASE): International Journal of Environmental Analytical Chemistry, v. 81, no. 4, p. 257280 .

Baehr, A.L., Kauffman, L.J., Charles, E.G., Baker, R.J., Stackelberg, P.E., Ayers, M.A., and Zapecza, O.S., 1999a, Sampling throughout the hydrologic cycle to characterize sources of volatile organic compounds in ground water, in Morganwalp, D.W., and Buxton, H.T., eds., Subsurface Contamination from Point Sources, U.S. Geological Survey Toxic Substances Hydrology Program-Proceedings of the technical meeting, Charleston, South Carolina, March 8-12, 1999: U.S. Geological Survey Water-Resources Investigations Report 99-4018C, v. 3, p. 21-36.

Baehr, A.L., Stackelberg, P.E., and Baker, R.J., 1999b, Evaluation of the atmosphere as a source of volatile organic compounds in shallow ground water: Water Resources Research, v. 35, no. 1, p. 127-136.

Bender, D.A., Zogorski, J.S., Luo, Wentai, Pankow, J.F., Majewski, M.S., and Baker, R.J., 2000, Atmospheric-water interaction of chloroform, toluene, and MTBE in small perennial urban streams, in Proceedings of the 93rd Annual Air \& Waste Management Association Conference, Salt Lake City, Utah, June 18-22, 2000.

Bigelow, D.S., 1984, Instruction manual: NADP/NTN site selection and installation: Fort Collins, Colorado, Colorado Stare University, Natural Resource Ecology laboratory, variously paged [available from National Atmospheric Deposition Program Office, Illinois State Water Survey, 2204 Griffith Drive, Champaign, IL. 61820]

Byard, J.L., 1999, Impact of SR125 vehicle emissions on the Sweetwater Reservoir-executive summary report: Chula Vista, Calif., Sweetwater Authority, 32p.

Cahill, J.D., Furlong, E.T., Burkhardt, M.R. Kolpin, D.W., and Anderson, L.G., 2004, Determination of pharmaceutical compounds in surface- and ground-water samples by solid-phase extraction and high-performance liquid chromatography/electrospray ionization mass spectrometry: Journal of Chromatography A, v. 1041, p. 171-180.

California Department of Transportation, 2001, State Route 125 (From I-905 to State Route 54): District 11 Fact Sheet, accessed December 14, 2001, at http://www.dot.ca.gov/ dost11/facts/125toll.htm (accessible at present [Jan. 28, 2008] at http://www.dot.ca.gov/dist11/facts/125spvly.pdf) 
California Environmental Protection Agency, 1986, California Safe Drinking Water and Toxic Enforcement Act of 1986 (Proposition 65): Health and Safety Code, chap. 6.6, sec. 25249.5-25249.13.

Childress, C.J.O., Foreman, W.T., Connor, B.F., and Maloney, T.J., 1999, New reporting procedures based on long-term method detection levels and some considerations for interpretations of water-quality data provided by the U.S. Geological Survey National Water Quality Laboratory: U.S. Geological Survey 99-193, 19 p., accessed March 2003 at http://water.usgs.gov/owq/OFR 99-193/index.html.

Connor, B.F., Rose, D.L., Noriega, M.C., Murtaugh, L.K., and Abney, S.R., 1998, Methods of analysis by the U.S. Geological Survey National Water Quality Laboratorydetermination of 86 volatile organic compounds in water by gas chromatography/mass spectrometry, including detections less than reporting limits: U.S. Geological Survey Open-File Report 97-829, 78 p.

Faires, L.M., 1993, Methods of analysis by the U.S. Geological Survey National Water Quality LaboratoryDetermination of metals in water by inductively coupled plasma-mass spectrometry: U.S. Geological Survey OpenFile Report 92-634, 28 p.

Foreman, W.T., Connor, B.F., Furlong, E.T., Vaught, D.G., and Merten, L.M., 1995, Methods of analysis by the U.S. Geological Survey National Water Quality Laboratory-determination of organochlorine pesticides and polychlorinated biphenyls in bottom sediment by dual capillary-column gas chromatography with electroncapture detection: U.S. Geological Survey Open-File Report 95-140, 78 p.

Foreman, W.T., Goolsby, D.A., Majewski, M.S., Wiebe, F.W., and Battaglin, W.A., 1997, Acetochlor and other herbicides in air and rain in the Midwestern United States [abs.], in Bridging the global environment: Technology, communication, and education: Society of Environmental Toxicology and Chemistry Annual Meeting, 18th, San Francisco, Calif., November 16-20,1997, Abstract Book no. 229, p. 45.

Foreman, W.T., Majewski, M.S., Goolsby, D.A., Wiebe, F.W., and Coupe, R.H., 2000, Pesticides in the atmosphere of the Mississippi River Valley, Part II-Air: Science of the Total Environment, v. 248, no. 2-3, p. 213-226.

Fram, M.S., Bergamaschi, B.B., Fujii, Roger, 2001, Improving water quality in Sweetwater Reservoir, San Diego County, California-Sources and mitigation strategies for trihalomethane (THM)-forming carbon: U.S. Geological Survey Fact sheet 112-01, 4p., accessed January 2007 at http://nwql.usgs.gov/fs/fs-112-01/.pdf (accessible at present [Jan. 28, 2008] at http://pubs.usgs.gov/fs/fs-112-01/).
Furlong, E.T., Anderson, B.D., Werner, S.L., Soliven, P.P., Coffey, L.J., and Burkhardt, M.R., 2001, Methods of analysis by the U.S. Geological Survey National Water Quality Laboratory-Determination of pesticides in water by graphitized carbon black solid-phase extraction and highperformance liquid chromatography/mass spectrometry: U.S. Geological Survey Water Resource Investigations Report 01-4134, 73 p., accessed March 2003 at http://nwql. usgs.gov/Public/pubs/WRIR01-4134.pdf.

Garbarino, J.R., and Struzeski, T.M., 1998, Methods of analysis by the U.S. Geological Survey National Water Quality Laboratory-Determination of elements in wholewater digests using inductively coupled plasma-optical emission spectrometry and inductively coupled plasmamass spectrometry: U.S. Geological Survey Open-File Report 98-165, $101 \mathrm{p}$.

Lindley, C.E., Stewart, J.T., and Sandstrom, M.W., 1996, Determination of low concentrations of acetochlor in water by automated solid-phase extraction and gas chromatography with mass selective detection: Journal of the Association of Official Analytical Chemists International, v. 79, no. 4, p. 962-966.

Madsen, J.E., Sandstrom, M.W., and Zaugg, S.D., 2003, Methods of analysis by the U.S. Geological Survey National Water Quality Laboratory-A method supplement for the determination of fipronil and degradates in water by gas chromatography/mass spectrometry: U.S. Geological Survey Open-File Report 02-462, 11 p., accessed March 2003 at http://nwql.usgs.gov/Public/pubs/OFR02-462/ OFR02-462.pdf

Majewski, M.S., Foreman, W.T., Goolsby, D.A., and Nakagaki, Naomi, 1998, Airborne pesticide residues along the Mississippi River: Environmental Science and Technology, v. 32, no. 23, p. 3689-3698.

Majewski, M.S., Sishu, J.S., Mendez, G.O., 2002, Waterquality monitoring of Sweetwater and Loveland Reservoirs, San Diego County, California_-Phase One results, 19981999: U.S. Geological Survey Open-File Report 02-186, $134 \mathrm{p}$.

Mendez, G.O., Foreman, W. T., Sidhu, J. S., and Majewski, M.S., 2007, Water- and Air-quality monitoring of the Sweetwater Reservoir watershed, San Diego County, California-Phase One results, continued, 1999-2001: U.S. Geological Survey Data Series 233, 270 p.

Ogden Environmental and Energy Services, 1997, SR-125 South Route alternatives: Potential air emissions impact on Sweetwater Reservoir: San Diego, Calif. [available from Sweetwater Authority, 505 Garrett Avenue, Chula Vista, CA 95912]. 
Olson, M.C., Iverson, J.L., Furlong, E.T., and Schroeder, M.P., 2004, Methods of analysis by the U.S. Geological Survey National Water Quality Laboratory-Determination of polycyclic aromatic hydrocarbon compounds in sediment by gas chromatography/mass spectrometry: U.S. Geological Survey Water-Resources Investigations Report 03-4318, $45 \mathrm{p}$.

Pankow, J.F., Luo, Wentai, Isabelle, L.M., Bender, D.A., and Baker, R.J., 1998, Determination of a wide range of volatile organic compounds in ambient air using multiabsorbent adsorption/ thermal desorption and gas chromatography/ mass spectrometry: Analytical Chemistry, v. 70, no. 24, p. 5213-5221.

Pankow, J.F., Luo, Wentai, Bender, D.A., Isabelle, L.M., Hollingsworth, J.S., Chen, C., Asher, W.E., and Zogorski, J.S., 2003, Concentrations and co-occurrence correlations of 88 volatile organic compounds (VOCs) in the ambient air of 13 semi-rural to urban locations in the United States: Atmospheric Environment, v. 37, no. 36, p. 5023-5046.

Sandstrom, M.W., 1995, Filtration of water-sediment samples for the determination of organic compounds: U.S. Geological Survey Water-Resources Investigations Report 95-4105, 13 p., accessed March 2003 at http://nwql.usgs. gov/Public/pubs/WRIR95-4105/WRIR95-4105.pdf

Sandstrom, M.W., Stroppel, M.E., Foreman, W.T., and Schroeder, M.P., 2001, Methods of analysis by the U.S. Geological Survey National Water Quality LaboratoryDetermination of moderate-use pesticides and selected degradates in water by $\mathrm{C}-18$ solid-phase extraction and gas chromatography/mass spectrometry: U.S. Geological Survey Water-Resources Investigations Report 01-4098, 70 p., accessed March 2003 at http://nwql.usgs.gov/Public/ pubs/WRIR01-4098.pdf

Shelton, L.R., 1994, Field guide for collecting and processing stream-water samples for the National Water-Quality Assessment Program: U.S. Geological Survey Open-File Report 94-455, 42 p.
Shelton, L.R., 1997, Field guide for collecting samples for analysis of volatile organic compounds in stream water for the National Water-Quality Assessment Program: U.S. Geological Survey Open-File Report 97-401, 14 p.

U.S. Environmental Protection Agency, 1999a, Compendium of methods for the determination of toxic organic compounds in ambient air-Compendium method TO-4A: Determination of pesticides and polychlorinated biphenyls in ambient air using high volume polyurethane foam (PUF) sampling followed by gas chromatographic/multi-detector detection (GC/MD) (2d ed.): Center for Environmental Research Information, Office of Research and Development EPA/625/R-96/010b, 49 p., accessed March 2003 at http:// www.epa.gov:80/ttnamti1/files/ambient/airtox/to-4ar2r.pdf

U.S. Environmental Protection Agency, 1999b, Compendium of methods for the determination of toxic organic compounds in ambient air-Compendium method TO-13A: Determination of polycyclic aromatic hydrocarbons (PAHs) in ambient air using gas chromatography/mass spectrometry (GC/MS) (2d ed.): Center for Environmental Research Information, Office of Research and Development EPA/625/ R-96/010b, 78 p., accessed March 2003 at http://www.epa. gov/ttn/amtic/files/ambient/airtox/to-13arr.pdf

Zaugg, S.D., Sandstrom, M.W., Smith, S.G., and Fehlberg, K.M., 1995, Methods of analysis by the U.S. Geological Survey National Water Quality Laboratory-Determination of pesticides in water by $\mathrm{C}-18$ solid-phase extraction and capillary-column gas chromatography/mass spectrometry with selected-ion monitoring: U.S. Geological Survey Open-File Report 95-181, 49 p., accessible at http://nwql. usgs.gov/Public/pubs/OFR95 181.pdf

Zaugg, S.D., Smith, S.G., Schroeder, M.P., Barber, L.B., and Burkhardt, M.R., 2002, Methods of analysis by the U.S. Geological Survey National Water Quality LaboratoryDetermination of wastewater compounds by polystyrenedivinylbenzene solid-phase extraction and capillary-column gas chromatography/mass spectrometry: U.S. Geological Survey Water-Resources Investigations Report 01-4186, 37 p., http://nwql.usgs.gov/Public/pubs/WRIR01-4186.html 


\section{Tables}


Table 2. Water-quality depth-profile data for dissolved oxygen, $\mathrm{pH}$, specific conductance, and temperature for each sampling site and period for the Sweetwater and Loveland Reservoirs, San Diego County, California.

[Time is denoted in 24-hour format. The five digit number in parentheses below each compound name, the parameter code, is used in the U.S. Geological Survey computerized system (National Water Information System) to uniquely identify a specific constituent or property. Sampling depth is in meters below the water surface. $\mathrm{m}$, meter; $\mathrm{mg} / \mathrm{L}$, milligram per liter; $\mu \mathrm{S} / \mathrm{cm}$, microsiemens per centimeter; ${ }^{\circ} \mathrm{C}$, degree Celsius; nd, no data]

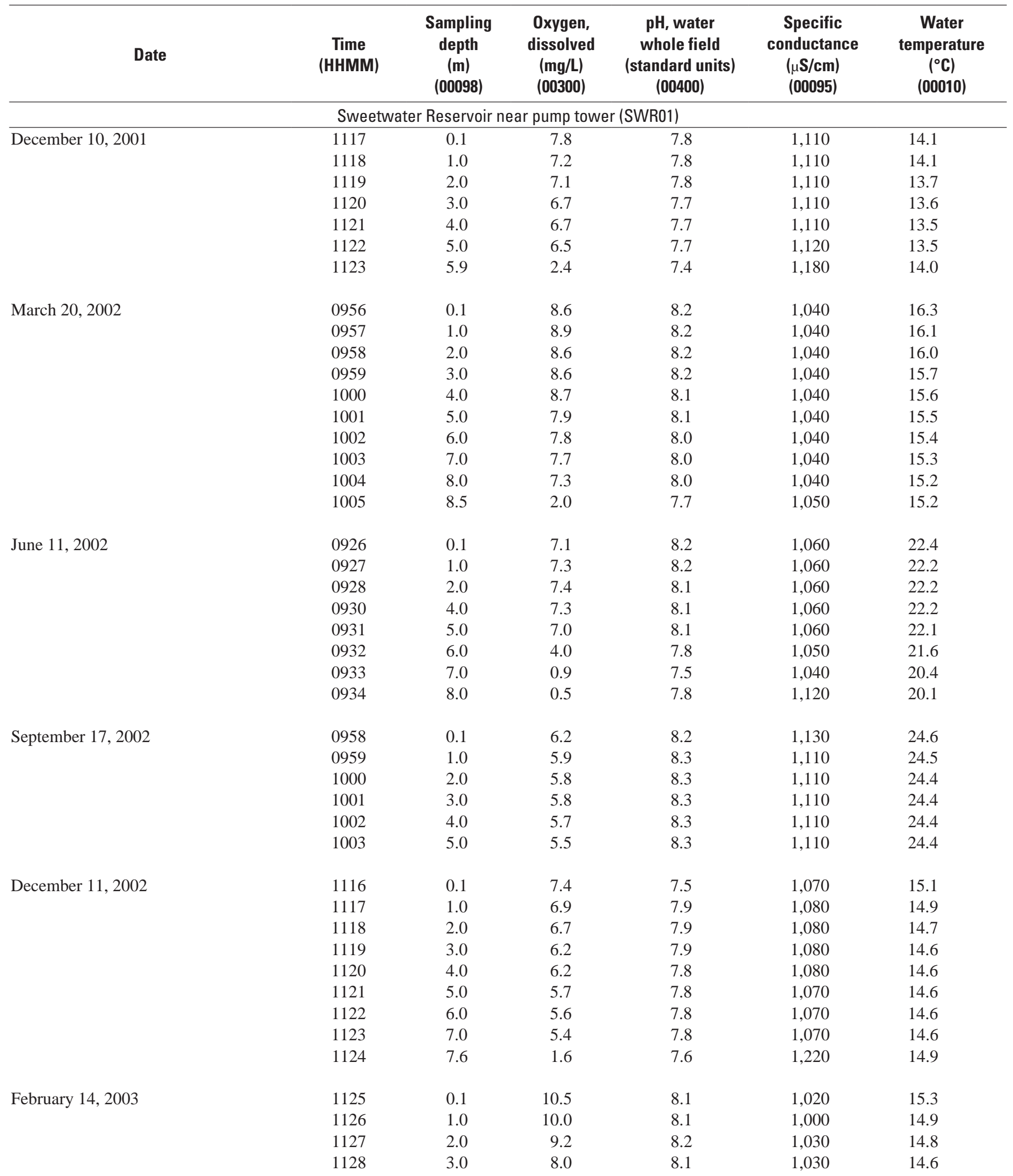


Table 2. Water-quality depth-profile data for dissolved oxygen, pH, specific conductance, and temperature for each sampling site and period for the Sweetwater and Loveland Reservoirs, San Diego County, California.-Continued

[Time is denoted in 24-hour format. The five digit number in parentheses below each compound name, the parameter code, is used in the U.S. Geological Survey computerized system (National Water Information System) to uniquely identify a specific constituent or property. Sampling depth is in meters below the water surface. $\mathrm{m}$, meter; $\mathrm{mg} / \mathrm{L}$, milligram per liter; $\mu \mathrm{S} / \mathrm{cm}$, microsiemens per centimeter; ${ }^{\circ} \mathrm{C}$, degree Celsius; nd, no data]

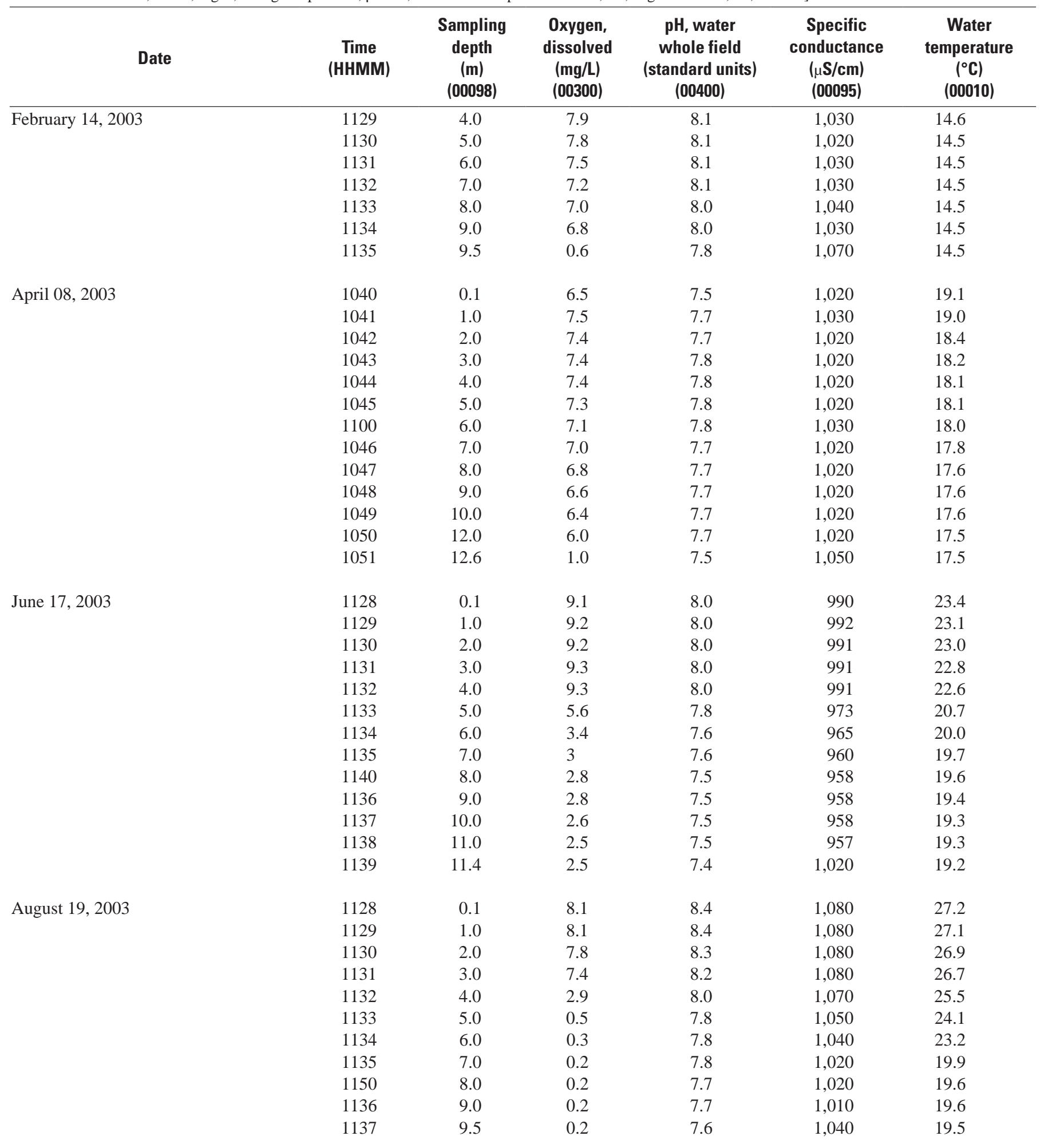


Table 2. Water-quality depth-profile data for dissolved oxygen, $\mathrm{pH}$, specific conductance, and temperature for each sampling site and period for the Sweetwater and Loveland Reservoirs, San Diego County, California.-Continued

[Time is denoted in 24-hour format. The five digit number in parentheses below each compound name, the parameter code, is used in the U.S. Geological Survey computerized system (National Water Information System) to uniquely identify a specific constituent or property. Sampling depth is in meters below the water surface. $\mathrm{m}$, meter; $\mathrm{mg} / \mathrm{L}$, milligram per liter; $\mu \mathrm{S} / \mathrm{cm}$, microsiemens per centimeter; ${ }^{\circ} \mathrm{C}$, degree Celsius; nd, no data]

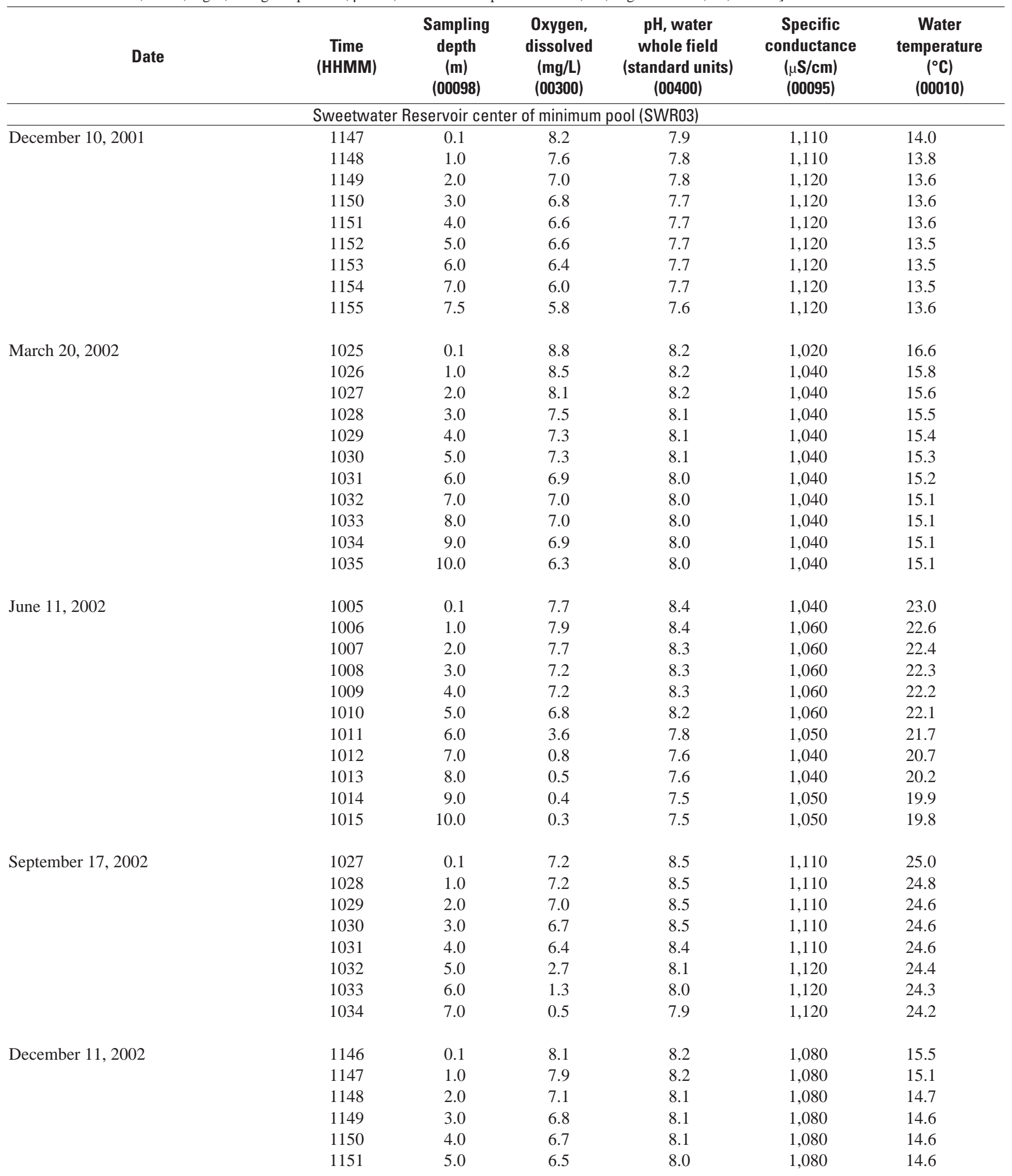


Table 2. Water-quality depth-profile data for dissolved oxygen, $\mathrm{pH}$, specific conductance, and temperature for each sampling site and period for the Sweetwater and Loveland Reservoirs, San Diego County, California._- Continued

[Time is denoted in 24-hour format. The five digit number in parentheses below each compound name, the parameter code, is used in the U.S. Geological Survey computerized system (National Water Information System) to uniquely identify a specific constituent or property. Sampling depth is in meters below the water surface. $\mathrm{m}$, meter; $\mathrm{mg} / \mathrm{L}$, milligram per liter; $\mu \mathrm{S} / \mathrm{cm}$, microsiemens per centimeter; ${ }^{\circ} \mathrm{C}$, degree Celsius; nd, no data]

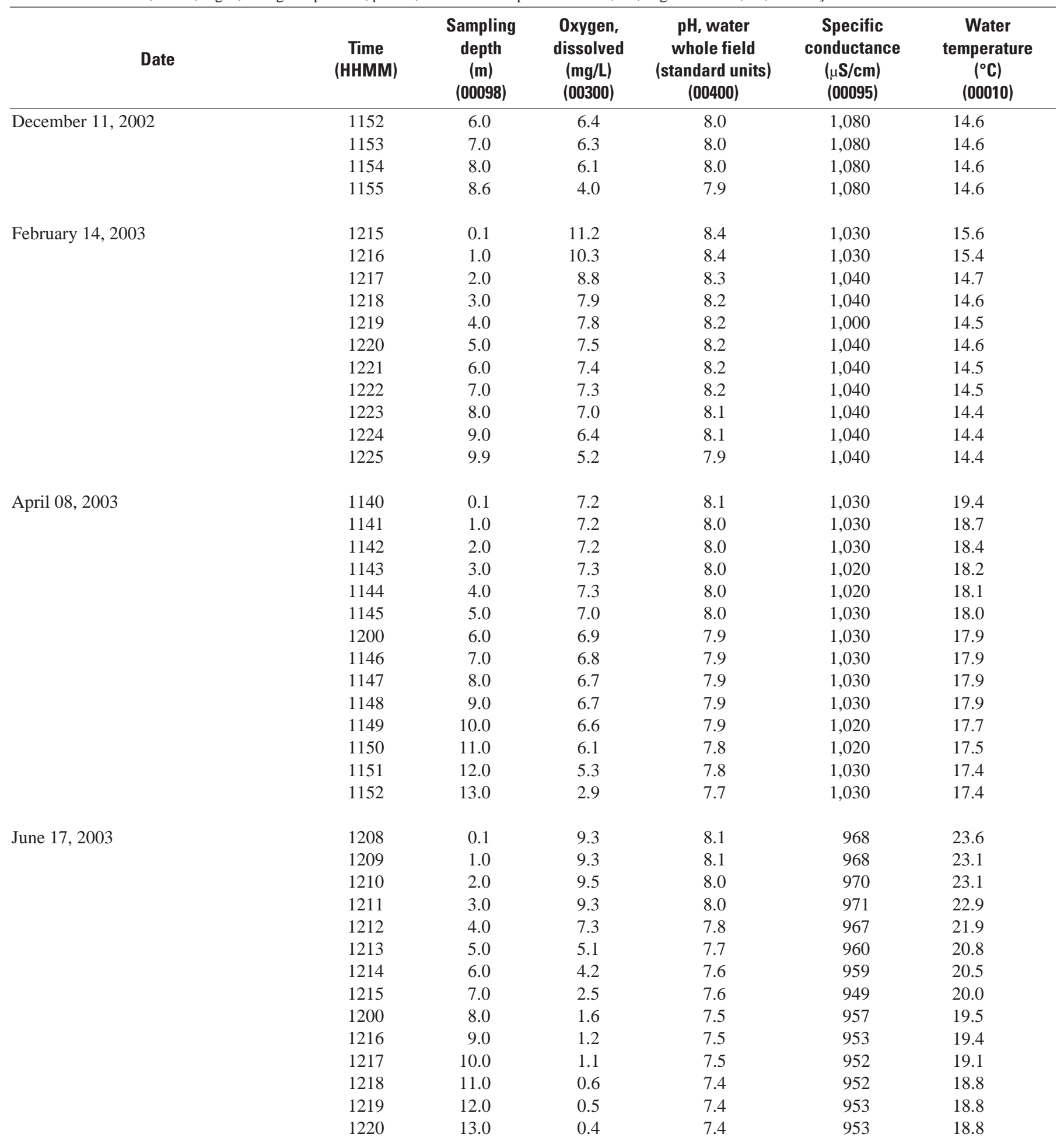


Table 2. Water-quality depth-profile data for dissolved oxygen, $\mathrm{pH}$, specific conductance, and temperature for each sampling site and period for the Sweetwater and Loveland Reservoirs, San Diego County, California.-Continued

[Time is denoted in 24-hour format. The five digit number in parentheses below each compound name, the parameter code, is used in the U.S. Geological Survey computerized system (National Water Information System) to uniquely identify a specific constituent or property. Sampling depth is in meters below the water surface. $\mathrm{m}$, meter; $\mathrm{mg} / \mathrm{L}$, milligram per liter; $\mu \mathrm{S} / \mathrm{cm}$, microsiemens per centimeter; ${ }^{\circ} \mathrm{C}$, degree Celsius; nd, no data]

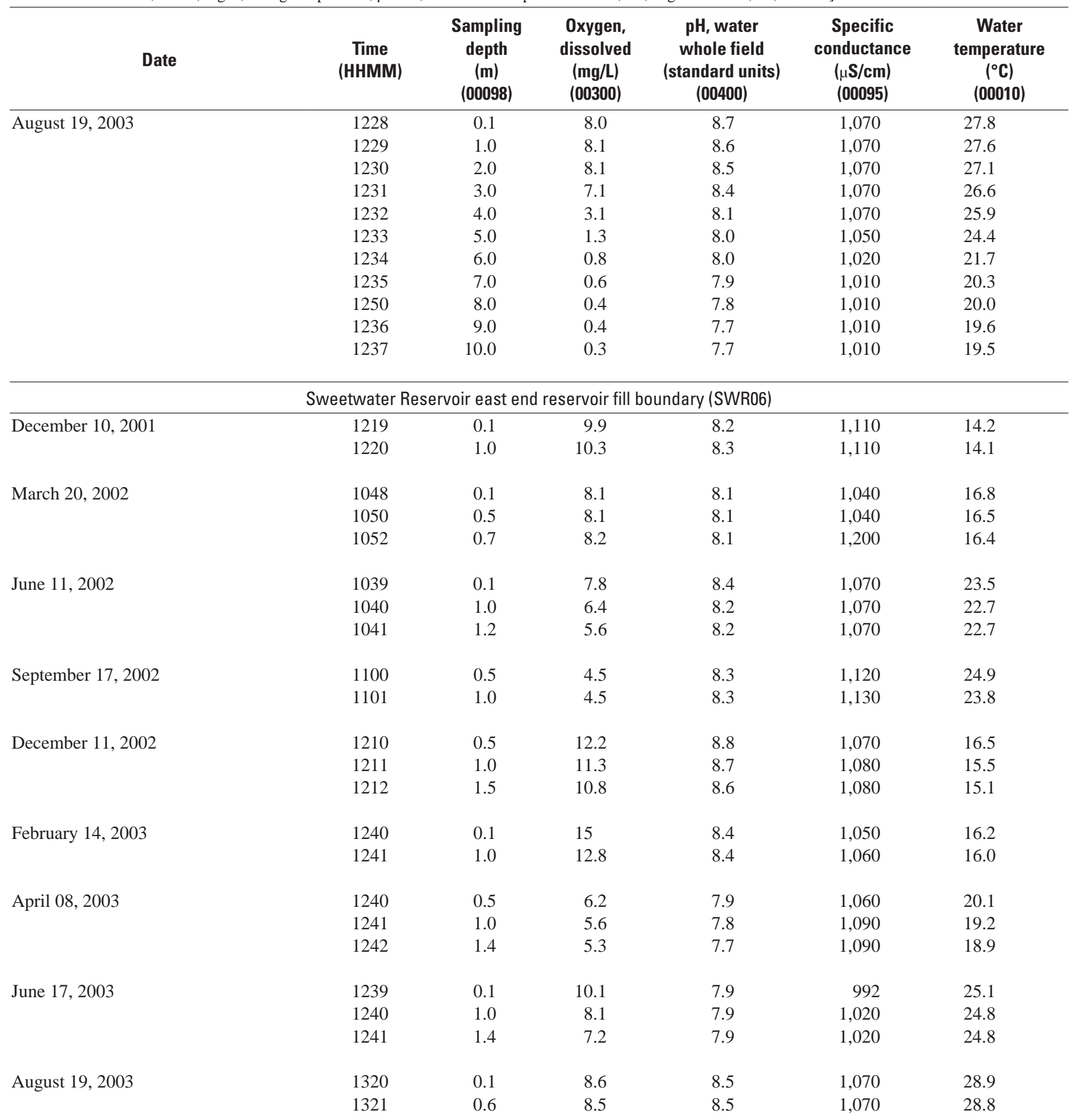


Table 2. Water-quality depth-profile data for dissolved oxygen, pH, specific conductance, and temperature for each sampling site and period for the Sweetwater and Loveland Reservoirs, San Diego County, California.-Continued

[Time is denoted in 24-hour format. The five digit number in parentheses below each compound name, the parameter code, is used in the U.S. Geological Survey computerized system (National Water Information System) to uniquely identify a specific constituent or property. Sampling depth is in meters below the water surface. $\mathrm{m}$, meter; $\mathrm{mg} / \mathrm{L}$, milligram per liter; $\mu \mathrm{S} / \mathrm{cm}$, microsiemens per centimeter; ${ }^{\circ} \mathrm{C}$, degree Celsius; nd, no data]

\begin{tabular}{|c|c|c|c|c|c|c|}
\hline Date & $\begin{array}{c}\text { Time } \\
\text { (HHMM) }\end{array}$ & $\begin{array}{l}\text { Sampling } \\
\text { depth } \\
\text { (m) } \\
(00098)\end{array}$ & $\begin{array}{c}\text { Oxygen, } \\
\text { dissolved } \\
\text { (mg/L) } \\
(00300)\end{array}$ & $\begin{array}{c}\text { pH, water } \\
\text { whole field } \\
\text { (standard units) } \\
\text { (00400) }\end{array}$ & $\begin{array}{c}\text { Specific } \\
\text { conductance } \\
(\mu \mathrm{S} / \mathrm{cm}) \\
(00095)\end{array}$ & $\begin{array}{c}\text { Water } \\
\text { temperature } \\
\left({ }^{\circ} \mathrm{C}\right) \\
(00010)\end{array}$ \\
\hline \multicolumn{7}{|c|}{ Sweetwater River at low-flow diversion dam above Sweetwater Reservoir (LFDD) } \\
\hline December 10, 2001 & 1420 & nd & nd & nd & nd & nd \\
\hline June 11,2002 & 1340 & nd & nd & nd & nd & nd \\
\hline September 17, 2002 & 1330 & nd & nd & nd & nd & nd \\
\hline December 12, 2002 & 1020 & 0.1 & 7.7 & 7.7 & 3,000 & 11.2 \\
\hline June 18, 2003 & 1400 & 0.1 & 5.4 & 7.6 & 2,850 & 20.2 \\
\hline August 20, 2003 & 1400 & 0.1 & 4.7 & 7.7 & 3,040 & 23.2 \\
\hline \multicolumn{7}{|c|}{ Loveland Reservoir near dam (LLR01) } \\
\hline \multirow[t]{11}{*}{ December 11, 2001} & 1019 & 0.1 & 3.5 & 7.4 & 576 & 12.8 \\
\hline & 1020 & 1.0 & 2.7 & 7.4 & 580 & 12.7 \\
\hline & 1021 & 2.0 & 2.4 & 7.4 & 579 & 12.7 \\
\hline & 1022 & 3.0 & 2.2 & 7.4 & 579 & 12.7 \\
\hline & 1023 & 4.0 & 2.1 & 7.4 & 579 & 12.7 \\
\hline & 1032 & 16.0 & 0.5 & 7.3 & 577 & 12.6 \\
\hline & 1033 & 18.0 & 0.4 & 7.3 & 573 & 12.2 \\
\hline & 1034 & 20.0 & 0.4 & 7.2 & 569 & 11.9 \\
\hline & 1035 & 22.0 & 0.3 & 7.2 & 568 & 11.7 \\
\hline & 1036 & 24.0 & 0.3 & 7.2 & 567 & 11.7 \\
\hline & 1037 & 26.0 & 0.3 & 7.2 & 567 & 11.7 \\
\hline \multirow[t]{9}{*}{ March 19, 2002} & 1108 & 0.1 & 8.8 & 8.2 & 561 & 13.7 \\
\hline & 1110 & 1.0 & 8.9 & 8.2 & 574 & 13.6 \\
\hline & 1112 & 2.0 & 8.9 & 8.2 & 583 & 12.8 \\
\hline & 1114 & 3.0 & 8.9 & 8.2 & 583 & 12.8 \\
\hline & 1116 & 4.0 & 8.9 & 8.2 & 582 & 12.8 \\
\hline & 1118 & 5.0 & 8.9 & 8.1 & 583 & 12.7 \\
\hline & 1120 & 6.0 & 8.8 & 8.1 & 583 & 12.6 \\
\hline & 1122 & 7.0 & 8.8 & 8.1 & 583 & 12.6 \\
\hline & 1124 & 8.0 & 8.8 & 8.1 & 584 & 12.6 \\
\hline
\end{tabular}


Table 2. Water-quality depth-profile data for dissolved oxygen, $\mathrm{pH}$, specific conductance, and temperature for each sampling site and period for the Sweetwater and Loveland Reservoirs, San Diego County, California.-Continued

[Time is denoted in 24-hour format. The five digit number in parentheses below each compound name, the parameter code, is used in the U.S. Geological Survey computerized system (National Water Information System) to uniquely identify a specific constituent or property. Sampling depth is in meters below the water surface. $\mathrm{m}$, meter; $\mathrm{mg} / \mathrm{L}$, milligram per liter; $\mu \mathrm{S} / \mathrm{cm}$, microsiemens per centimeter; ${ }^{\circ} \mathrm{C}$, degree Celsius; nd, no data]

\begin{tabular}{|c|c|c|c|c|c|c|}
\hline Date & $\begin{array}{c}\text { Time } \\
\text { (HHMM) }\end{array}$ & $\begin{array}{l}\text { Sampling } \\
\text { depth } \\
\text { (m) } \\
(00098)\end{array}$ & $\begin{array}{c}\text { Oxygen, } \\
\text { dissolved } \\
\text { (mg/L) } \\
(00300)\end{array}$ & $\begin{array}{c}\text { pH, water } \\
\text { whole field } \\
\text { (standard units) } \\
(00400)\end{array}$ & $\begin{array}{c}\text { Specific } \\
\text { conductance } \\
(\mu \mathrm{S} / \mathrm{cm}) \\
(00095)\end{array}$ & $\begin{array}{c}\text { Water } \\
\text { temperature } \\
\left({ }^{\circ} \mathrm{C}\right) \\
(00010)\end{array}$ \\
\hline \multirow[t]{9}{*}{ March 19, 2002} & 1126 & 9.0 & 8.5 & 8.0 & 584 & 12.3 \\
\hline & 1128 & 10.0 & 7.7 & 7.9 & 585 & 12.0 \\
\hline & 1129 & 12.0 & 6.5 & 7.7 & 585 & 11.6 \\
\hline & 1134 & 16.0 & 5.6 & 7.6 & 584 & 11.2 \\
\hline & 1136 & 18.0 & 5.5 & 7.6 & 584 & 11.2 \\
\hline & 1138 & 20.0 & 5.4 & 7.6 & 584 & 11.1 \\
\hline & 1140 & 22.0 & 5.4 & 7.6 & 584 & 11.1 \\
\hline & 1142 & 24.0 & 5.1 & 7.5 & 583 & 11.0 \\
\hline & 1144 & 26.0 & 4.8 & 7.5 & 583 & 10.9 \\
\hline \multirow{12}{*}{ June 12, 2002} & 1114 & 1.0 & 11.4 & 9.2 & 557 & 22.5 \\
\hline & 1130 & 2.0 & 11.0 & 9.1 & 552 & 22.4 \\
\hline & 1115 & 3.0 & 10.7 & 9.1 & 553 & 22.2 \\
\hline & 1116 & 4.0 & 1.4 & 8.0 & 588 & 19.3 \\
\hline & 1117 & 5.0 & 0.8 & 7.9 & 592 & 17.7 \\
\hline & 1118 & 6.0 & 0.6 & 7.8 & 595 & 16.8 \\
\hline & 1119 & 7.0 & 0.5 & 7.7 & 596 & 15.9 \\
\hline & 1120 & 8.0 & 0.4 & 7.6 & 597 & 15.2 \\
\hline & 1121 & 9.0 & 0.4 & 7.6 & 599 & 14.1 \\
\hline & 1122 & 10.0 & 0.3 & 7.6 & 600 & 13.3 \\
\hline & 1123 & 12.0 & 0.3 & 7.6 & 599 & 12.5 \\
\hline & 1124 & 14.0 & 0.3 & 7.5 & 597 & 12.2 \\
\hline \multirow[t]{17}{*}{ September 17, 2002} & 1047 & 0.1 & 6.8 & 8.7 & 604 & 23.2 \\
\hline & 1048 & 1.0 & 6.9 & 8.7 & 602 & 23.2 \\
\hline & 1049 & 2.0 & 6.8 & 8.6 & 602 & 23.1 \\
\hline & 1110 & 3.0 & 6.8 & 8.6 & 602 & 23.0 \\
\hline & 1050 & 4.0 & 6.8 & 8.6 & 602 & 23.0 \\
\hline & 1051 & 5.0 & 6.8 & 8.6 & 602 & 23.0 \\
\hline & 1052 & 6.0 & 1.4 & 8.2 & 602 & 22.1 \\
\hline & 1053 & 7.0 & 0.4 & 7.8 & 601 & 17.0 \\
\hline & 1054 & 8.0 & 0.3 & 7.7 & 602 & 15.4 \\
\hline & 1055 & 9.0 & 0.2 & 7.7 & 601 & 14.3 \\
\hline & 1056 & 10.0 & 0.2 & 7.6 & 602 & 13.8 \\
\hline & 1057 & 12.0 & 0.1 & 7.7 & 598 & 12.9 \\
\hline & 1058 & 14.0 & 0.1 & 7.6 & 600 & 12.4 \\
\hline & 1059 & 16.0 & 0.1 & 7.5 & 601 & 12.3 \\
\hline & 1100 & 18.0 & 0.1 & 7.5 & 601 & 12.2 \\
\hline & 1101 & 20.0 & 0.1 & 7.5 & 602 & 12.2 \\
\hline & 1102 & 22.0 & 0.1 & 7.5 & 600 & 12.1 \\
\hline
\end{tabular}


Table 2. Water-quality depth-profile data for dissolved oxygen, pH, specific conductance, and temperature for each sampling site and period for the Sweetwater and Loveland Reservoirs, San Diego County, California._- Continued

[Time is denoted in 24-hour format. The five digit number in parentheses below each compound name, the parameter code, is used in the U.S. Geological Survey computerized system (National Water Information System) to uniquely identify a specific constituent or property. Sampling depth is in meters below the water surface. $\mathrm{m}$, meter; $\mathrm{mg} / \mathrm{L}$, milligram per liter; $\mu \mathrm{S} / \mathrm{cm}$, microsiemens per centimeter; ${ }^{\circ} \mathrm{C}$, degree Celsius; nd, no data]

\begin{tabular}{|c|c|c|c|c|c|c|}
\hline Date & $\begin{array}{c}\text { Time } \\
\text { (HHMM) }\end{array}$ & $\begin{array}{l}\text { Sampling } \\
\text { depth } \\
(\mathrm{m}) \\
(00098)\end{array}$ & $\begin{array}{c}\text { Oxygen, } \\
\text { dissolved } \\
\text { (mg/L) } \\
(00300)\end{array}$ & $\begin{array}{c}\text { pH, water } \\
\text { whole field } \\
\text { (standard units) } \\
(00400)\end{array}$ & $\begin{array}{c}\text { Specific } \\
\text { conductance } \\
(\mu \mathrm{S} / \mathrm{cm}) \\
(00095)\end{array}$ & $\begin{array}{c}\text { Water } \\
\text { temperature } \\
\left({ }^{\circ} \mathrm{C}\right) \\
(00010)\end{array}$ \\
\hline \multirow[t]{2}{*}{ September 17, 2002} & 1103 & 24.0 & 0.1 & 7.5 & 601 & 12.1 \\
\hline & 1104 & 26.0 & 0.1 & 7.4 & 602 & 12.0 \\
\hline \multirow{14}{*}{ December 12, 2002} & 1131 & 2.0 & 5.2 & 7.7 & 608 & 13.0 \\
\hline & 1132 & 3.0 & 5.0 & 7.7 & 608 & 13.0 \\
\hline & 1133 & 4.0 & 4.9 & 7.7 & 608 & 13.0 \\
\hline & 1134 & 5.0 & 4.9 & 7.7 & 608 & 13.0 \\
\hline & 1135 & 6.0 & 4.8 & 7.7 & 608 & 13.0 \\
\hline & 1136 & 7.0 & 4.8 & 7.7 & 608 & 12.9 \\
\hline & 1140 & 12.0 & 4.5 & 7.7 & 609 & 12.9 \\
\hline & 1141 & 14.0 & 4.5 & 7.7 & 608 & 12.9 \\
\hline & 1142 & 16.0 & 4.4 & 7.7 & 608 & 12.9 \\
\hline & 1143 & 18.0 & 4.4 & 7.7 & 608 & 12.9 \\
\hline & 1144 & 20.0 & 4.3 & 7.7 & 608 & 12.9 \\
\hline & 1145 & 22.0 & 4.2 & 7.6 & 608 & 12.9 \\
\hline & 1146 & 24.0 & 0.2 & 7.3 & 602 & 12.4 \\
\hline & 1147 & 26.0 & 0.1 & 7.3 & 602 & 12.4 \\
\hline \multirow[t]{13}{*}{ February 12, 2003} & 1048 & 0.2 & 8.4 & 7.9 & 617 & 13.3 \\
\hline & 1049 & 1.0 & 8.2 & 7.9 & 618 & 13.3 \\
\hline & 1050 & 2.0 & 8.1 & 7.9 & 618 & 13.3 \\
\hline & 1058 & 10.0 & 7.1 & 7.8 & 619 & 12.4 \\
\hline & 1059 & 12.0 & 7.1 & 7.8 & 618 & 12.3 \\
\hline & 1100 & 14.0 & 7.0 & 7.8 & 618 & 12.2 \\
\hline & 1101 & 16.0 & 5.1 & 7.7 & 616 & 12.0 \\
\hline & 1102 & 18.0 & 2.7 & 7.5 & 616 & 11.8 \\
\hline & 1103 & 20.0 & 2.7 & 7.5 & 614 & 11.7 \\
\hline & 1104 & 22.0 & 2.5 & 7.5 & 614 & 11.6 \\
\hline & 1105 & 24.0 & 2.4 & 7.5 & 614 & 11.6 \\
\hline & 1106 & 26.0 & 2.1 & 7.4 & 615 & 11.6 \\
\hline & 1107 & 28.0 & 1.6 & 7.4 & 621 & 11.6 \\
\hline \multirow[t]{6}{*}{ April 09, 2003* } & 1201 & 0.1 & 11.3 & 8.1 & 626 & 17.4 \\
\hline & 1202 & 1.0 & 12.1 & 8.2 & 627 & 16.9 \\
\hline & 1203 & 2.0 & 12.3 & 8.3 & 628 & 16.7 \\
\hline & 1204 & 3.0 & 12.0 & 8.3 & 628 & 16.6 \\
\hline & 1205 & 4.0 & 11.4 & 8.2 & 627 & 15.4 \\
\hline & 1206 & 5.0 & 6.3 & 8.2 & 628 & 11.0 \\
\hline
\end{tabular}


Table 2. Water-quality depth-profile data for dissolved oxygen, $\mathrm{pH}$, specific conductance, and temperature for each sampling site and period for the Sweetwater and Loveland Reservoirs, San Diego County, California.-Continued

[Time is denoted in 24-hour format. The five digit number in parentheses below each compound name, the parameter code, is used in the U.S. Geological Survey computerized system (National Water Information System) to uniquely identify a specific constituent or property. Sampling depth is in meters below the water surface. $\mathrm{m}$, meter; $\mathrm{mg} / \mathrm{L}$, milligram per liter; $\mu \mathrm{S} / \mathrm{cm}$, microsiemens per centimeter; ${ }^{\circ} \mathrm{C}$, degree Celsius; nd, no data]

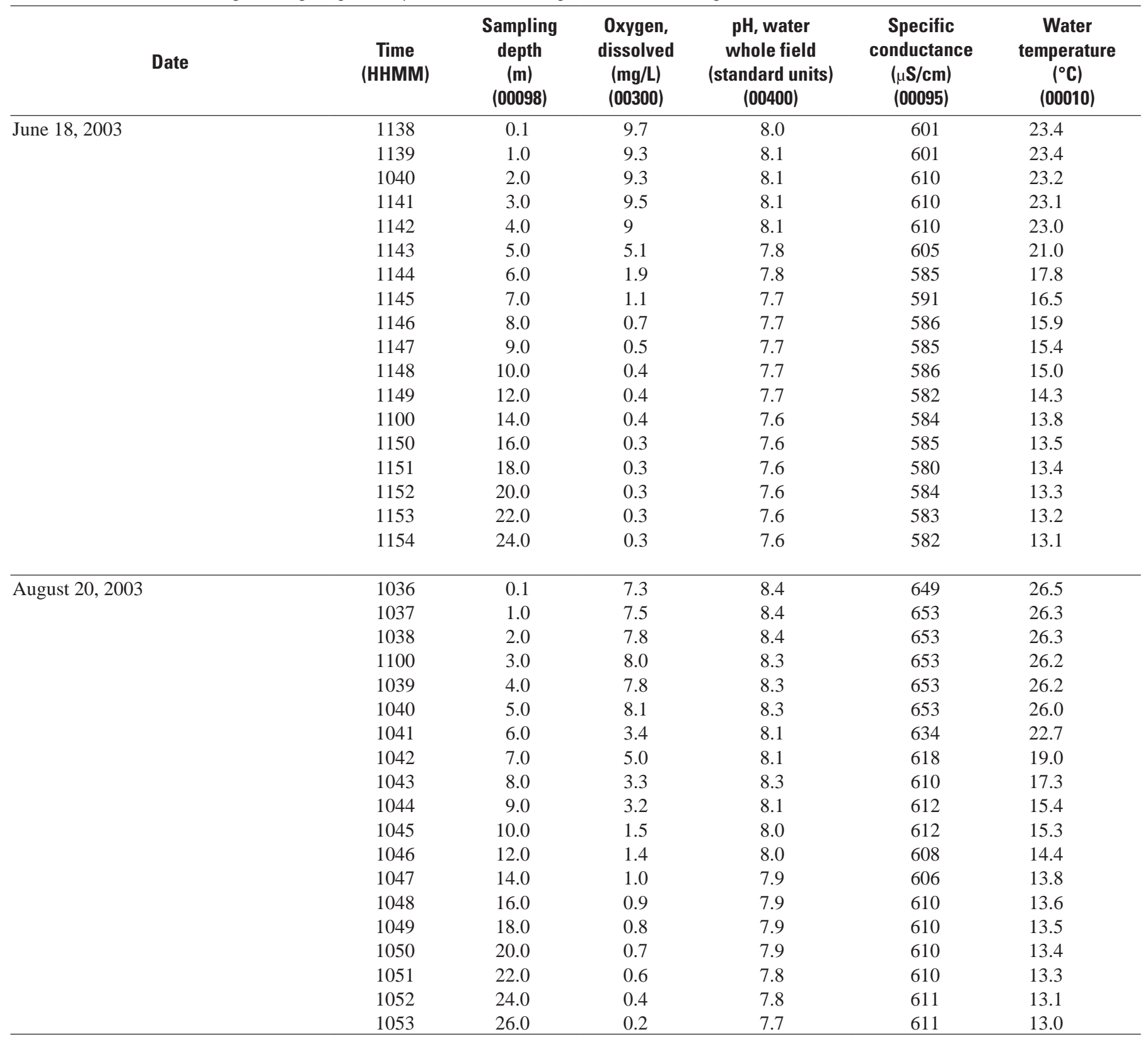

*Probe malfunctioned at 5 meters; total depth was 24 meters. 
Table 3A. Volatile organic compounds analyzed in whole-water samples from the Sweetwater Reservoir watershed, San Diego County, California.

[The parameter code is used in the U.S. Geological Survey computerized data system (National Water Information System) to uniquely identify a specific constituent or property; LRL, laboratory reporting level. LRL values are reported as micrograms per liter ( $\mu \mathrm{g} / \mathrm{L})$ unless noted]

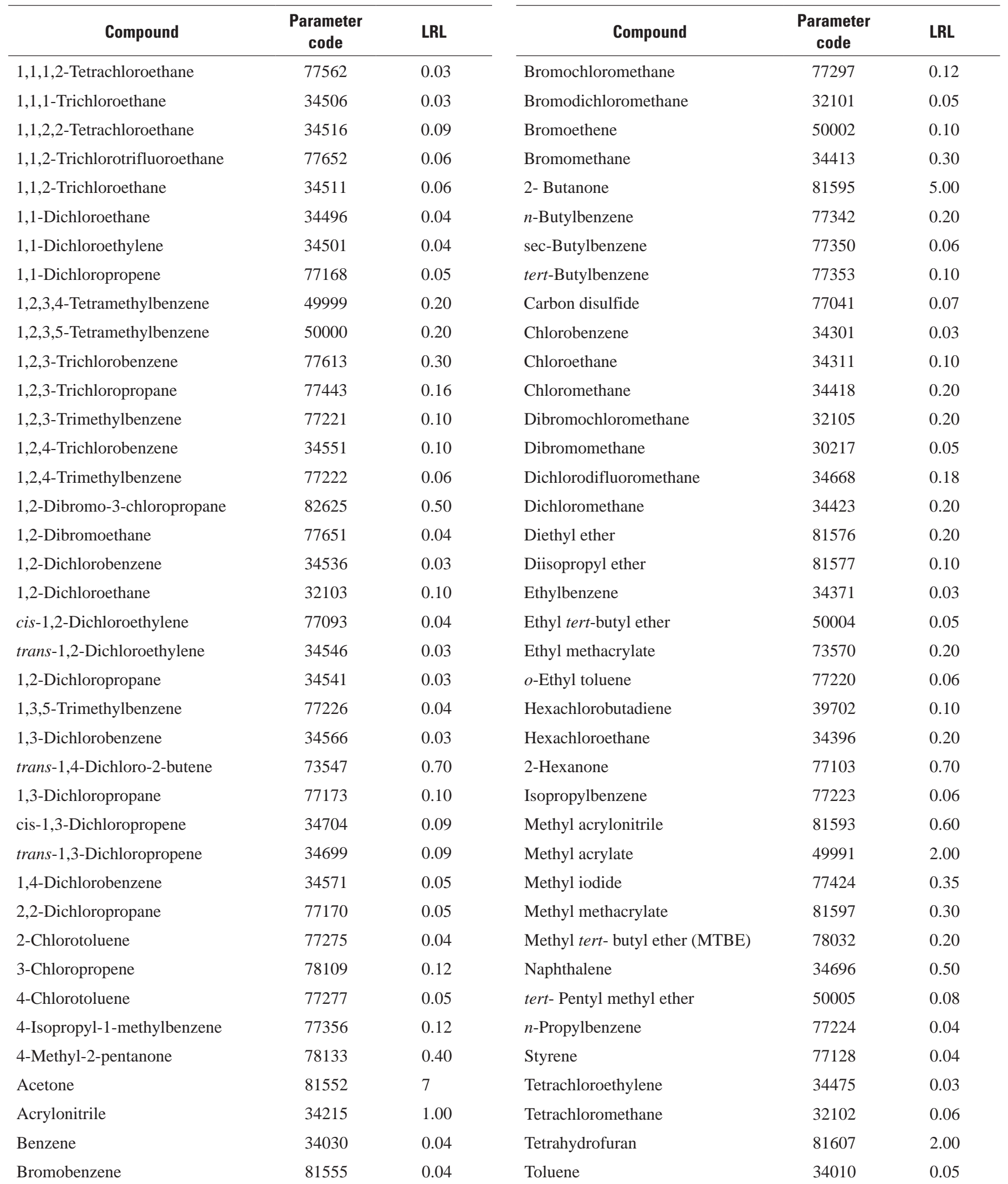


Table 3A. Volatile organic compounds analyzed in whole-water samples from the Sweetwater Reservoir watershed, San Diego County, California.-Continued

[The parameter code is used in the U.S. Geological Survey computerized data system (National Water Information System) to uniquely identify a specific constituent or property; LRL, laboratory reporting level. LRL values are reported as micrograms per liter $(\mu \mathrm{g} / \mathrm{L})$ unless noted]

\begin{tabular}{lcc}
\hline \multicolumn{1}{c}{ Compound } & $\begin{array}{c}\text { Parameter } \\
\text { code }\end{array}$ & LRL \\
\hline Tribromomethane & 32104 & 0.10 \\
Trichloroethylene & 39108 & 0.04 \\
Trichlorofluoromethane & 34488 & 0.09 \\
Trichloromethane (chloroform) & 32106 & 0.20 \\
Vinyl chloride & 39175 & 0.10 \\
$m$ - and $p$-Xylene & 85795 & 0.06 \\
$o$-Xylene & 77135 & 0.07 \\
& & \\
1,4-Bromofluorobenzene, surrogate & 99834 & percent \\
1,2-Dichloroethane- $d 4$, surrogate & 99832 & percent \\
Toluene- $d 8$, surrogate & 99833 & percent \\
\hline
\end{tabular}




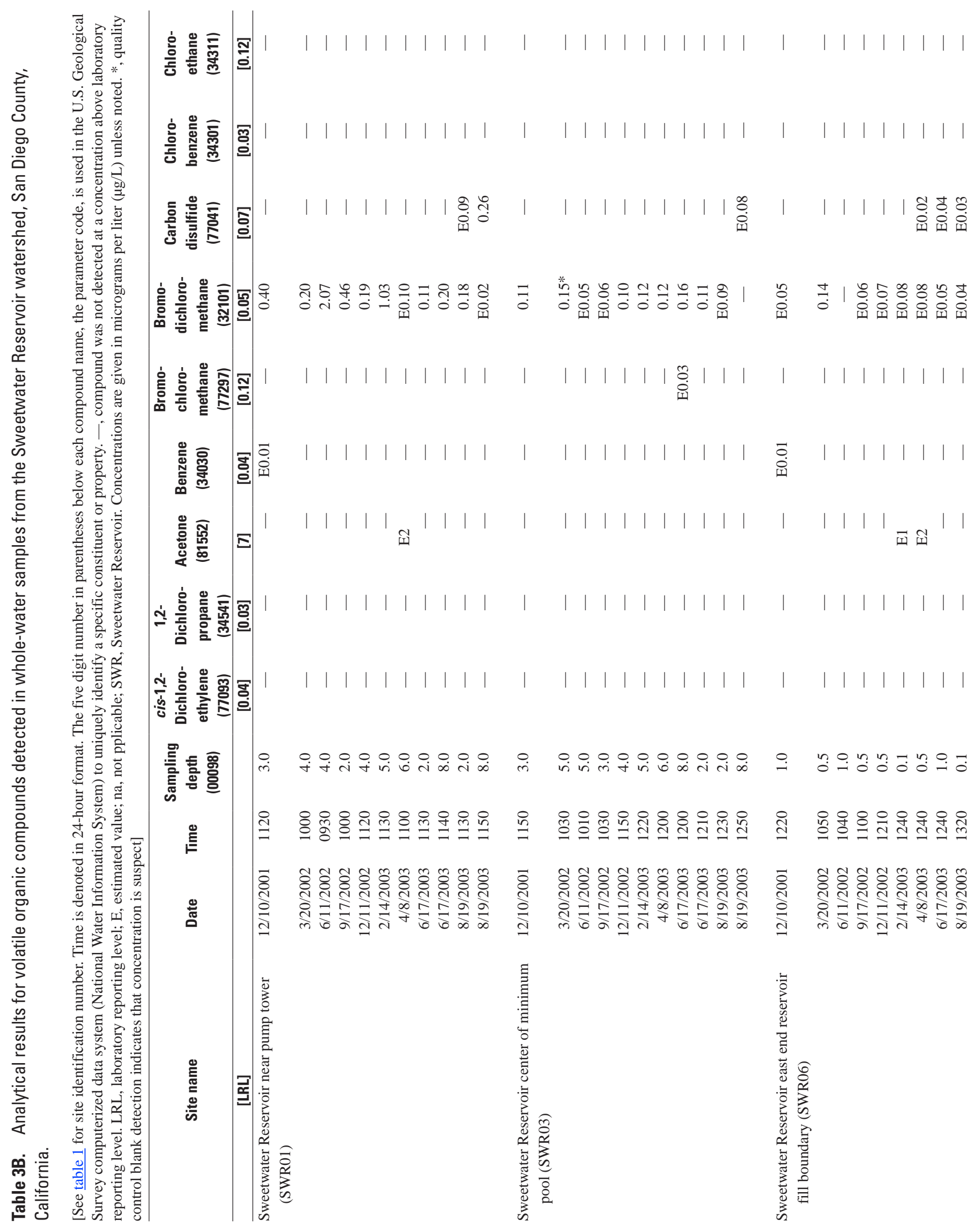




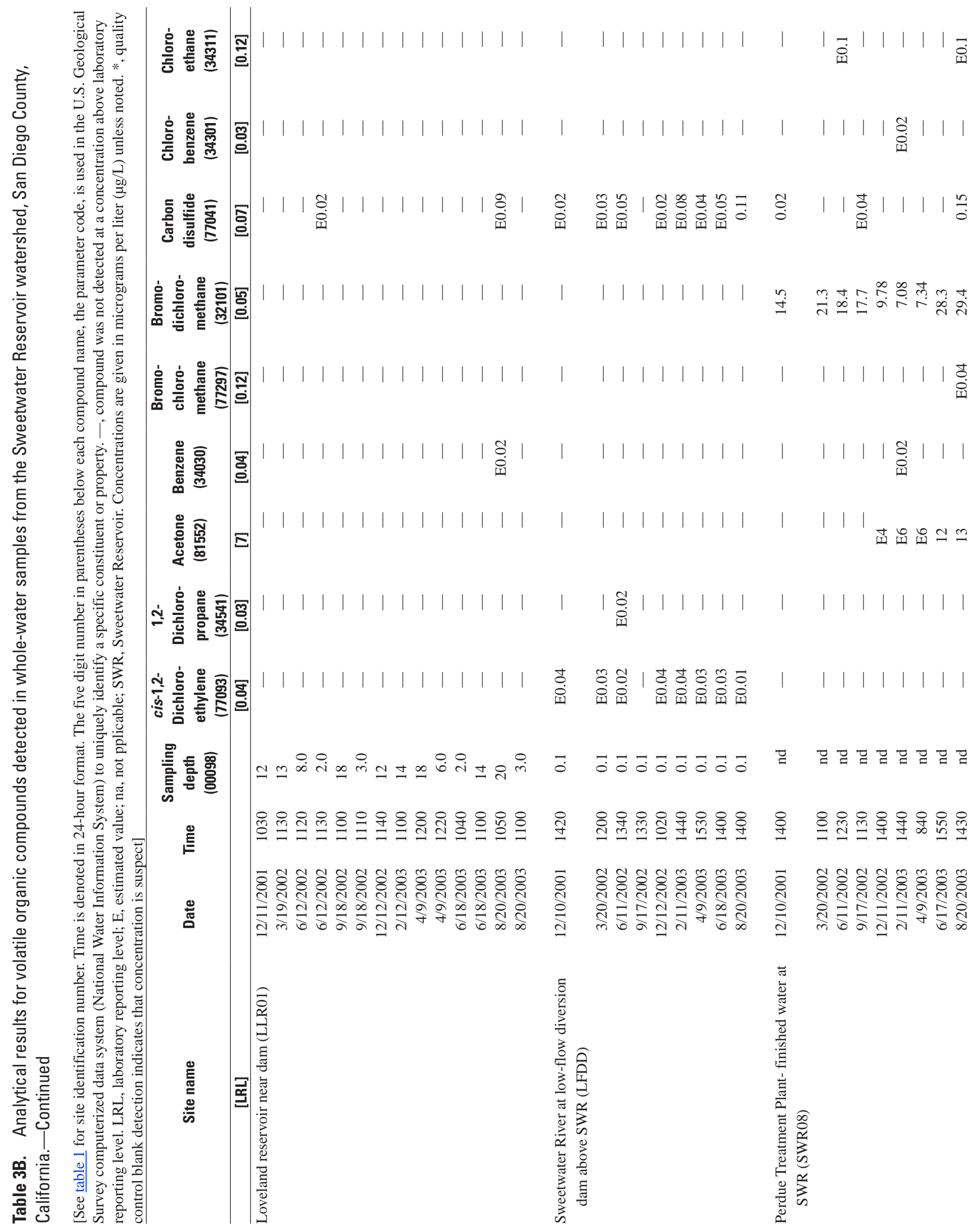




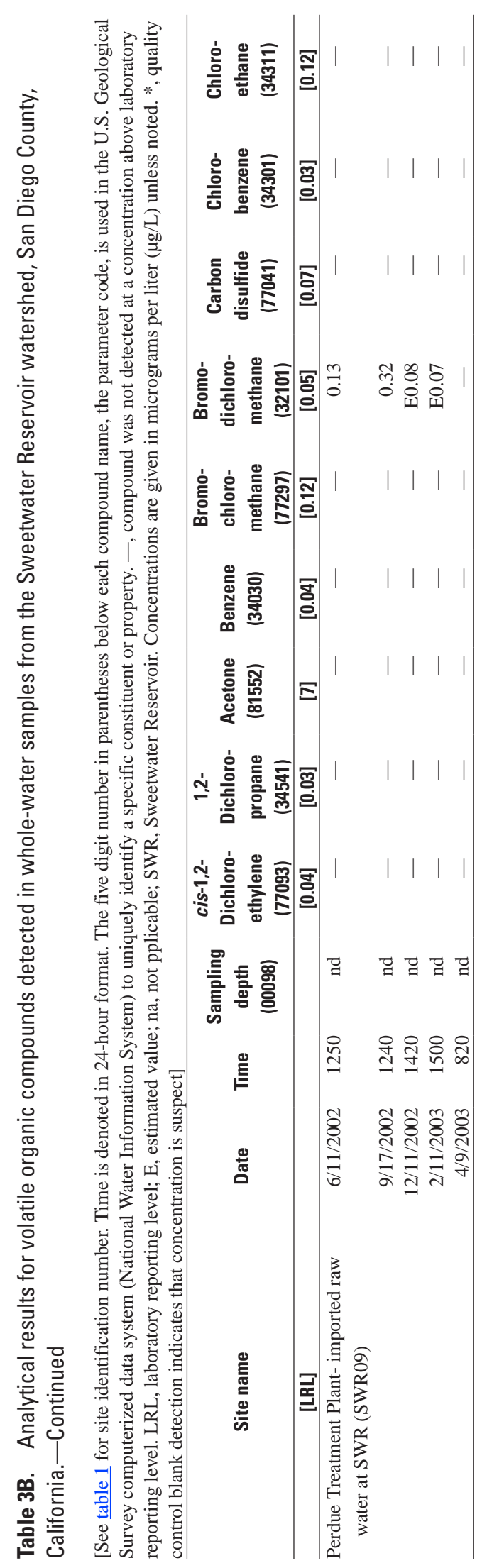


Table 4A. Polycyclic aromatic hydrocarbon compounds analyzed in whole-water samples from the Sweetwater Reservoir watershed, San Diego County, California.

[The parameter code is used in the U.S. Geological Survey (USGS) computerized data system (National Water Information System) to uniquely identify a specific constituent or property. Laboratory reporting level (LRL) values are reported as micrograms per liter unless noted]

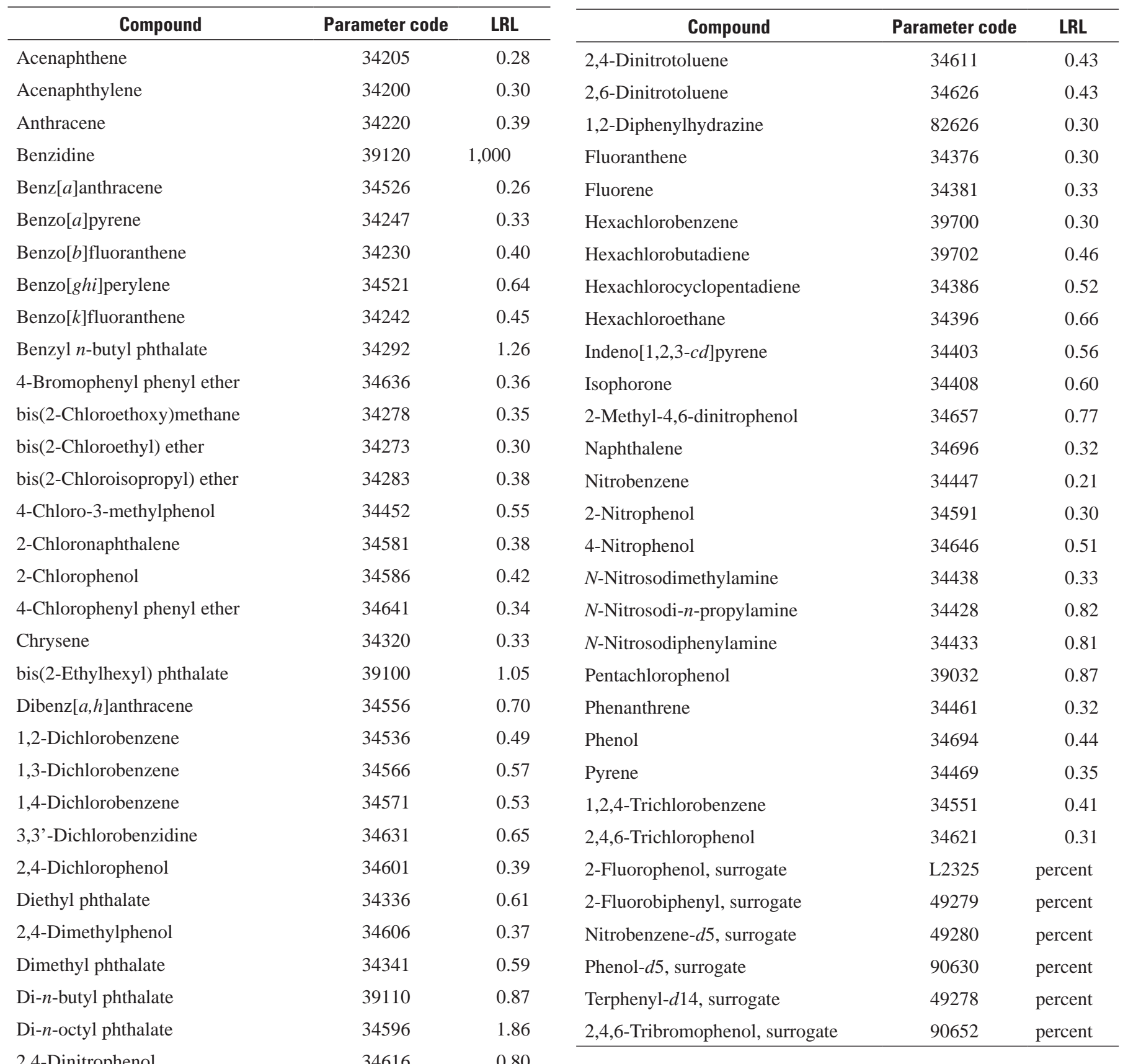


Table 4B. Analytical results for polycyclic aromatic hydrocarbon concentrations in whole-water samples from the Sweetwater Reservoir watershed, San Diego County, California.

[See table 1 for site identification number. Time is denoted in 24-hour scale. The five digit number in parentheses below the compound name, the parameter code, is used in the U.S. Geological Survey computerized data system (National Water Information System) to uniquely identify a specific constituent or property. - , compound was not detected at a concentration above laboratory reporting level. LRL, laboratory reporting level; E, estimated value; na, not applicable. Concentrations are reported as micrograms per liter $(\mu \mathrm{g} / \mathrm{L})$ unless noted]

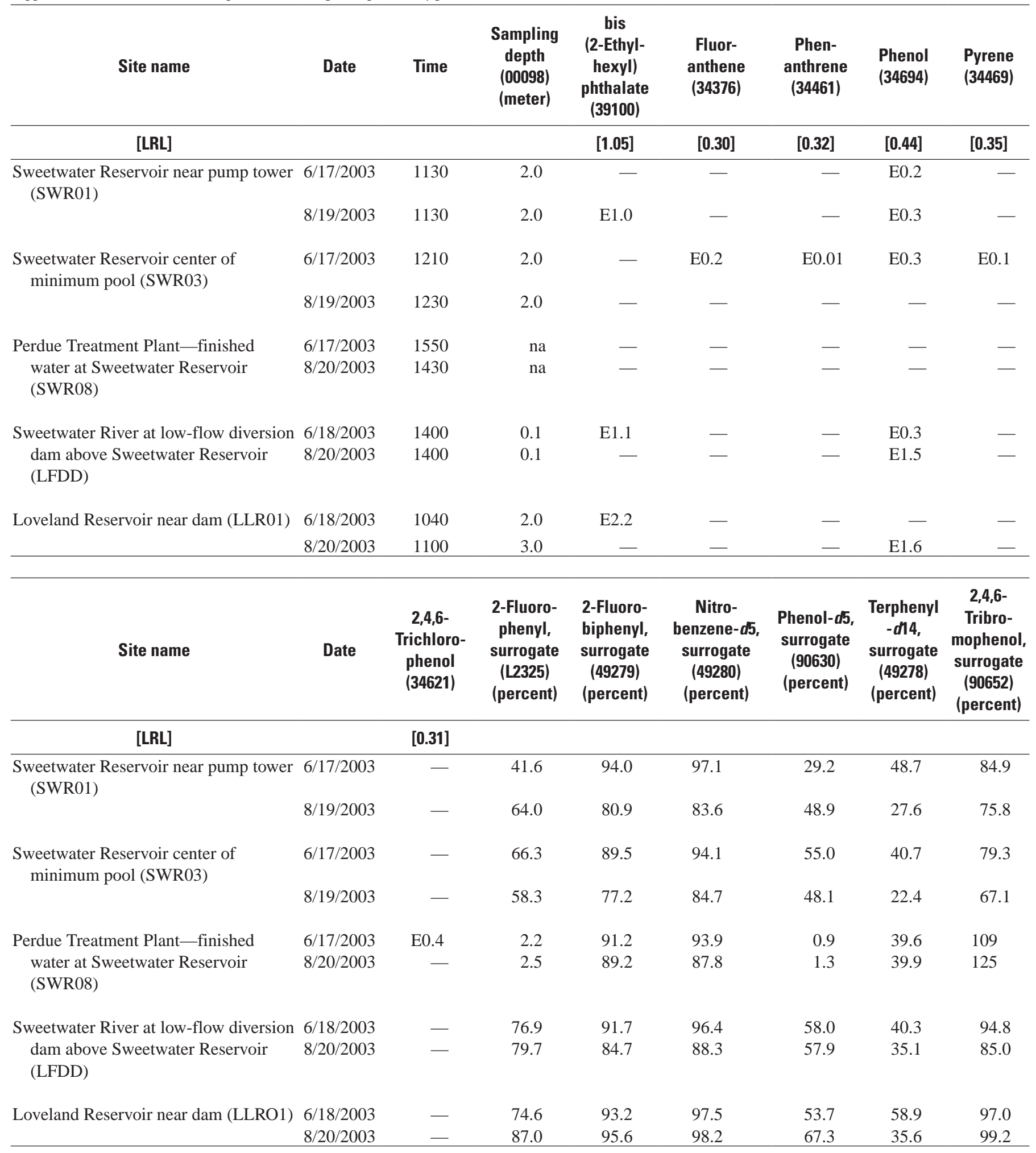


Table 5A. Pesticide compounds analyzed in filtered-water samples, using U.S.Geological Survey's National Water Quality Laboratory schedule 2001, from the Sweetwater Reservoir watershed, San Diego County, California.

The parameter code is used in the U.S. Geological Survey computerized data system (National Water Information System) to uniquely identify a specific constituent or property. LRL, laboratory reporting level; LRL values reported as micrograms per liter unless noted]

\begin{tabular}{|c|c|c|c|c|c|}
\hline Compound & Parameter code & LRL & Compound & Parameter code & LRL \\
\hline Acetochlor & 49260 & 0.006 & Metolachlor & 39415 & 0.013 \\
\hline Alachlor & 46342 & 0.004 & Metribuzin & 82630 & 0.006 \\
\hline Atrazine & 39632 & 0.007 & Molinate & 82671 & 0.002 \\
\hline Azinphos-methyl & 82686 & 0.005 & Napropamide & 82684 & 0.007 \\
\hline Benfluralin & 82673 & 0.010 & Parathion & 39542 & 0.010 \\
\hline Butylate & 04028 & 0.002 & Parathion-methyl & 82667 & 0.006 \\
\hline Carbaryl & 82680 & 0.041 & Pebulate & 82669 & 0.004 \\
\hline Carbofuran & 82674 & 0.020 & Pendimethalin & 82683 & 0.022 \\
\hline Chlorpyrifos & 38933 & 0.005 & cis- Permethrin & 82687 & 0.006 \\
\hline Cyanazine & 04041 & 0.018 & Phorate & 82664 & 0.011 \\
\hline Dacthal (DCPA) & 82682 & 0.003 & Prometon & 04037 & 0.010 \\
\hline$p, p^{\prime}-\mathrm{DDE}$ & 34653 & 0.003 & Propachlor & 04024 & 0.010 \\
\hline Deethylatrazine & 04040 & 0.006 & Propanil & 82679 & 0.011 \\
\hline Diazinon & 39572 & 0.005 & Propargite & 82685 & 0.020 \\
\hline Dieldrin & 39381 & 0.005 & Pronyzamide & 82676 & 0.004 \\
\hline 2,6-Diethylaniline & 82660 & 0.006 & Simazine & 04035 & 0.005 \\
\hline Disulfoton & 82677 & 0.020 & Tebuthiuron & 82670 & 0.020 \\
\hline EPTC & 82668 & 0.002 & Terbacil & 82665 & 0.034 \\
\hline Ethalfluralin & 82663 & 0.009 & Terbufos & 82675 & 0.020 \\
\hline Ethoprophos & 82672 & 0.005 & Thiobencarb & 82681 & 0.005 \\
\hline Fonofos & 04095 & 0.003 & Tri-allate & 82678 & 0.002 \\
\hline$\alpha-\mathrm{HCH}$ & 34253 & 0.005 & Trifluralin & 82661 & 0.009 \\
\hline Lindane & 39341 & 0.004 & Diazinon- $d 10$, surrogate & 91063 & percent \\
\hline Linuron & 82666 & 0.035 & $\alpha-\mathrm{HCH}-d 6$, surrogate & 91065 & percent \\
\hline
\end{tabular}

Malathion

$39532 \quad 0.027$




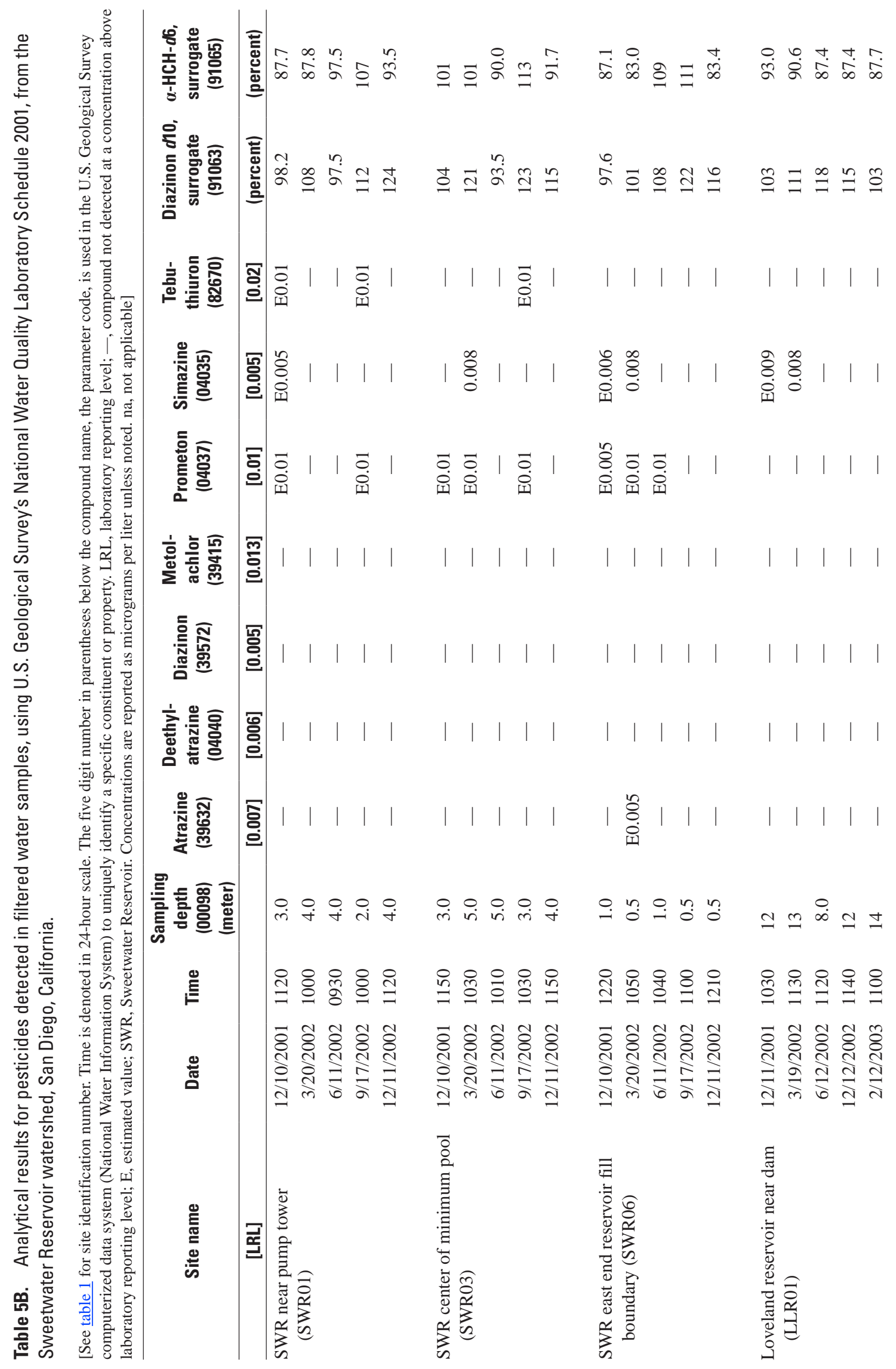




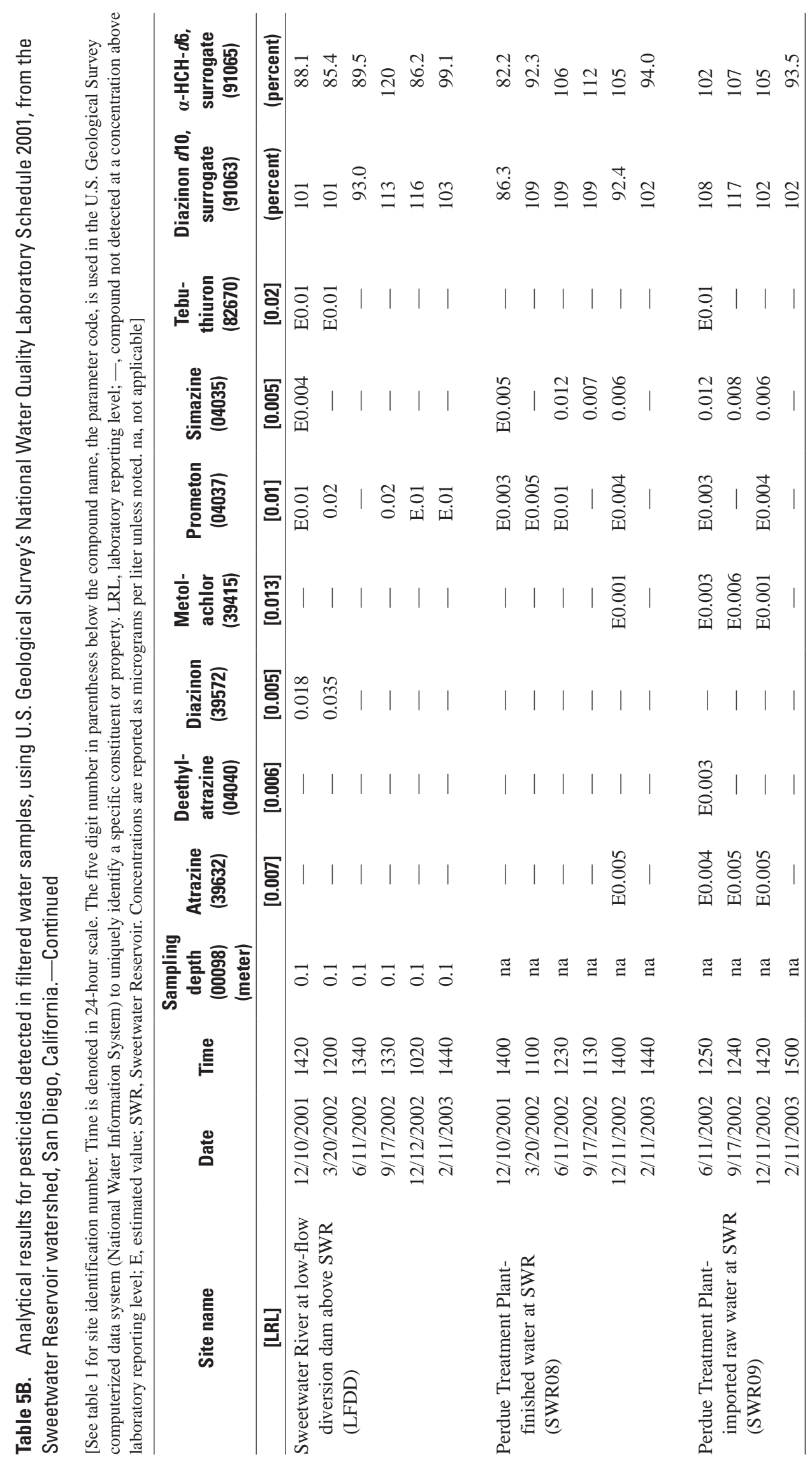


Table 6A. Pesticide compounds analyzed in filtered-water samples, using U.S. Geological Survey's National Water Quality Laboratory schedule 2002, from the Sweetwater Reservoir watershed, San Diego County, California.

[The parameter code is used in the U.S. Geological Survey computerized data system (National Water Information System) to uniquely identify a specific constituent or property. LRL, laboratory reporting level; LRL values reported as micrograms per liter $(\mu \mathrm{g} / \mathrm{L})$ unless noted]

\begin{tabular}{|c|c|c|c|c|c|}
\hline Compound & Parameter code & LRL & Compound & Parameter code & LRL \\
\hline 2-Amino- $N$-isopropylbenzamide & 61617 & 0.005 & Fonofos oxygen analog & 61649 & 0.002 \\
\hline Azinphos-methyl-oxon & 61635 & 0.016 & Iprodione & 61593 & 1.42 \\
\hline Bifenthrin & 61580 & 0.005 & Isofenphos & 61594 & 0.003 \\
\hline hexanol & & & Malaoxon & 61652 & 0.008 \\
\hline 2-Chloro-2,6-diethylacetanilide & 61618 & 0.005 & Metalaxyl & 61596 & 0.005 \\
\hline 4-Chloro-2-methyl phenol & 61633 & 0.006 & Methidathion & 61598 & 0.006 \\
\hline Cycloate & 04031 & 0.005 & 2,2-dimethyl-(1-cyclopropane)- & & \\
\hline Cyfluthrin & 61585 & 0.008 & carboxylate & & \\
\hline$\lambda$-Cyhalothrin & 61595 & 0.009 & $t$-Methyl-3-(2,2-dichlorovinyl)- & 79843 & 0.013 \\
\hline Cypermethrin & 61586 & 0.009 & 2,2-dimethyl-(1-cyclopropane)- & & \\
\hline 2,5-Dichloroaniline & 61614 & 0.026 & carboxylate & & \\
\hline 3,4-Dichloroaniline & 61625 & 0.008 & Myclobutanil & 61599 & 0.008 \\
\hline Dimethoate & 82662 & 0.006 & Paraoxon-methyl & 61664 & 0.030 \\
\hline E-Dimethomorph & 79844 & 0.020 & Pendimethalin & 61665 & 0.143 \\
\hline Z-Dimethomorph & 79845 & 0.046 & Phorate oxon & 61666 & 0.097 \\
\hline Disulfoton sulfone & 61640 & 0.016 & Phosmet & 61601 & 0.008 \\
\hline Disulfoton sulfoxide & 61641 & 0.002 & Phosmet oxon & 61668 & 0.055 \\
\hline Endosulfan ether & 61642 & 0.004 & Profenofos & 61603 & 0.006 \\
\hline$\alpha$-Endosulfan & 34362 & 0.005 & Prometryn & 04036 & 0.006 \\
\hline$\beta$-Endosulfan & 34357 & 0.014 & cis-Propiconazole & 79846 & 0.008 \\
\hline Endosulfan sulfate & 61590 & 0.006 & trans-Propiconazole & 79847 & 0.013 \\
\hline Ethion & 82346 & 0.004 & Sulfotepp & 61605 & 0.003 \\
\hline Ethion monoxon & 61644 & 0.034 & Sulprofos & 38716 & 0.024 \\
\hline Fenthion & 38801 & 0.015 & 3-Trifluoromethylaniline & 61630 & 0.011 \\
\hline Fenthion sulfoxide & 61647 & 0.008 & Diazinon- $d 10$ surrogate & 99223 & Percent \\
\hline Flumetralin & 61592 & 0.004 & $\alpha-\mathrm{HCH}-d 6$ surrogate & 99224 & Percent \\
\hline
\end{tabular}


Table 6B. Analytical results for pesticide compounds detected in filtered-water samples, using U.S. Geological Survey's National Water Quality Laboratory schedule 2002, from the Sweetwater Reservoir watershed, San Diego County, California.

[See table 1 for site identification number. Time is denoted in 24-hour scale. The five digit number in parentheses below each compound name, the parameter code, is used in the U.S. Geological Survey computerized data system (National Water Information System) to uniquely identify a specific constituent or property. LRL, laboratory reporting level; SWR, Sweetwater Reservoir; E, estimated value; concentrations reported as micrograms per liter ( $\mu \mathrm{g} / \mathrm{L}$ ) unless noted]

\begin{tabular}{|c|c|c|c|c|c|c|}
\hline Site name & Date & Time & $\begin{array}{c}\text { Hexazinone } \\
\text { (04025) }\end{array}$ & $\begin{array}{c}\text { Prometryn } \\
\text { (04036) }\end{array}$ & $\begin{array}{c}\text { Diazinon- } \\
\text { d10, } \\
\text { surrogate } \\
\text { (percent) } \\
\text { (99223) }\end{array}$ & $\begin{array}{c}\alpha-\mathrm{HCH}-d 6 \\
\text { surrogate } \\
\text { (percent) } \\
(34230)\end{array}$ \\
\hline [LRL] & & & {$[0.007]$} & {$[0.041]$} & & \\
\hline $\begin{array}{l}\text { Perdue Treatment Plant - finished water at SWR } \\
\text { (SWR08) }\end{array}$ & 06/11/2002 & 1250 & Е 0.009 & E0.003 & 84.8 & 102.8 \\
\hline
\end{tabular}


Table 7A. Pesticide compounds analyzed in filtered-water samples, using U.S. Geological Survey's National Water Quality Laboratory schedule 2003, from the Sweetwater Reservoir watershed, San Diego County, California.

[The parameter code is used in the U.S. Geological Survey computerized data system (National Water Information System) to uniquely identify a specific constituent or property. LRL, laboratory reporting level; LRL values reported as micrograms per liter unless noted]

\begin{tabular}{|c|c|c|c|c|c|}
\hline Compound & Parameter code & LRL & Compound & Parameter code & LRL \\
\hline Acetochlor & 49260 & 0.006 & Fipronil sulfone & 62168 & 0.005 \\
\hline Alachlor & 46342 & 0.004 & Fonofos & 04095 & 0.003 \\
\hline Atrazine & 39632 & 0.007 & Fonofos oxygen analog & 61649 & 0.002 \\
\hline Azinphos-methyl oxygen analog & 61635 & 0.02 & Iprodione & 61593 & 1.42 \\
\hline Benfluralin & 82673 & 0.10 & Isofenphos & 91594 & 0.003 \\
\hline Carbaryl & 82680 & 0.041 & Malaoxon & 61652 & 0.008 \\
\hline 4-Chloro-2-methylphenol & 61633 & 0.006 & Metalaxyl & 61596 & 0.005 \\
\hline Chlorpyrifos & 38933 & 0.005 & Methidathion & 61598 & 0.006 \\
\hline Chlorpyrifos oxygen analog & 61636 & 0.06 & Metolachlor & 39415 & 0.013 \\
\hline Cyfluthrin & 61585 & 0.008 & Metribuzin & 82630 & 0.006 \\
\hline Cypermethrin & 61586 & 0.009 & Myclobutanil & 61599 & 0.008 \\
\hline Dacthal (DCPA) & 82682 & 0.003 & 1-Naphthol & 49295 & 0.09 \\
\hline 3,4-Dichloroaniline & 61625 & 0.004 & Phorate & 82664 & 0.011 \\
\hline Dichlorvos & 38775 & 0.012 & Phorate oxygen analog & 61666 & 0.10 \\
\hline Dicrotophos & 38454 & 0.08 & Phosmet & 61601 & 0.008 \\
\hline Dieldrin & 39381 & 0.005 & Phosmet oxygen analog & 61668 & 0.060 \\
\hline 2,6-Diethylaniline & 82660 & 0.006 & Prometon & 04037 & 0.01 \\
\hline Dimethoate & 82662 & 0.006 & Prometryn & 04036 & 0.005 \\
\hline Ethion & 82346 & 0.004 & Pronamide & 82676 & 0.004 \\
\hline Ethion monoxon & 61644 & 0.03 & Simazine & 04035 & 0.005 \\
\hline 2-Ethyl-6-methylaniline & 61620 & 0.004 & Tebuthiuron & 82670 & 0.02 \\
\hline \multirow{2}{*}{$\begin{array}{l}\text { 2-[(2-Ethyl-6-methylphenyl)- } \\
\text { amino]-1-propanol }\end{array}$} & \multirow{2}{*}{61615} & \multirow{2}{*}{0.10} & Terbufos & 82675 & 0.02 \\
\hline & & & Terbufos oxygen analog sulfone & 61674 & 0.07 \\
\hline
\end{tabular}


Table 7B. Analytical results for pesticide compounds detected in filtered-water samples, using U.S. Geological Survey's National Water Quality Laboratory schedule 2003, from the Sweetwater Reservoir watershed, San Diego County, California.

[See table 1 for site identification number. Time is denoted in 24-hour format. The five digit number in parentheses below each compound name, the parameter code, is used in the U.S. Geological Survey computerized data system (National Water Information System) to uniquely identify a specific constituent or property. LRL, laboratory reporting level; —, compound not detected at a concentration above laboratory reporting level; E, estimated value; SWR, Sweetwater Reservoir; NA, not analyzed; na, not applicable; concentrations reported as micrograms per liter unless noted]

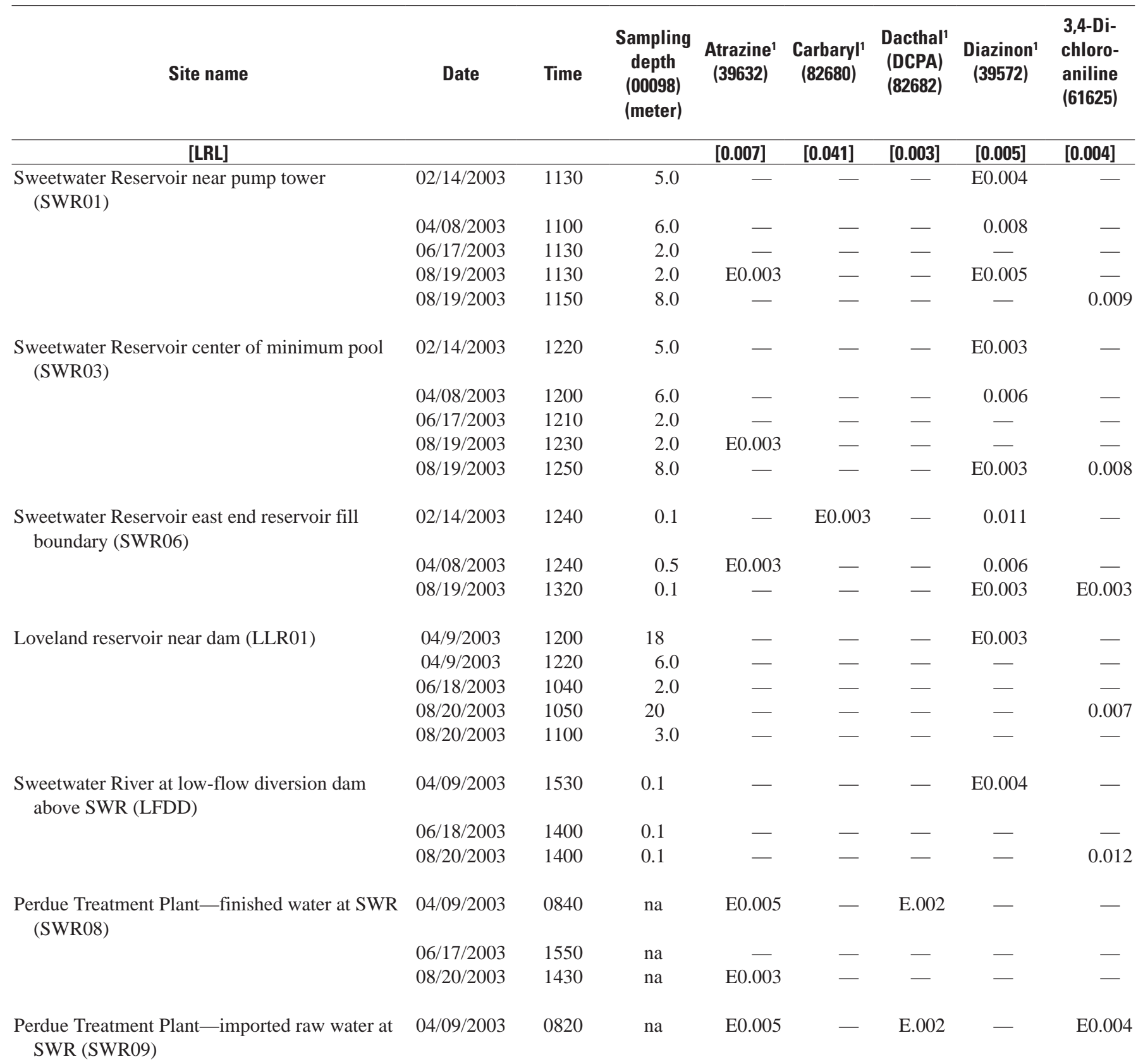


Table 7B. Analytical results for pesticide compounds detected in filtered-water samples, using U.S. Geological Survey's National Water Quality Laboratory schedule 2003, from the Sweetwater Reservoir watershed, San Diego County, California.-Continued

[See table 1 for site identification number. Time is denoted in 24-hour format. The five digit number in parentheses below each compound name, the parameter code, is used in the U.S. Geological Survey computerized data system (National Water Information System) to uniquely identify a specific constituent or property. LRL, laboratory reporting level; - , compound not detected at a concentration above laboratory reporting level; E, estimated value; SWR, Sweetwater Reservoir; NA, not analyzed; na, not applicable; concentrations reported as micrograms per liter unless noted]

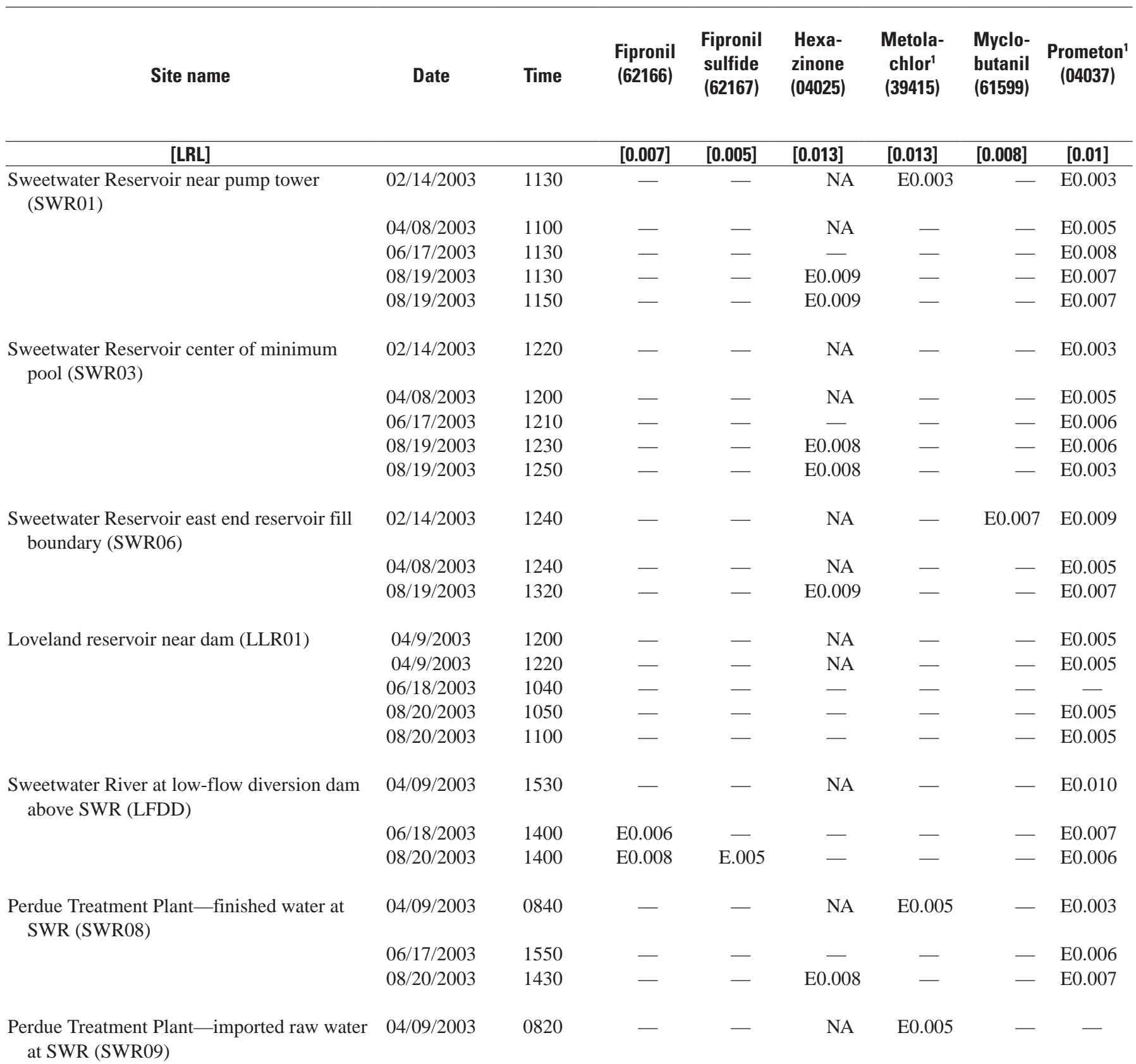


Table 7B. Analytical results for pesticide compounds detected in filtered-water samples, using U.S. Geological Survey's National Water Quality Laboratory schedule 2003, from the Sweetwater Reservoir watershed, San Diego County, California.—Continued

[See table 1 for site identification number. Time is denoted in 24-hour format. The five digit number in parentheses below each compound name, the parameter code, is used in the U.S. Geological Survey computerized data system (National Water Information System) to uniquely identify a specific constituent or property. LRL, laboratory reporting level; —, compound not detected at a concentration above laboratory reporting level; E, estimated value; SWR, Sweetwater Reservoir; NA, not analyzed; na, not applicable; concentrations reported as micrograms per liter unless noted]

\begin{tabular}{|c|c|c|c|c|c|c|c|}
\hline Site name & Date & Time & $\begin{array}{l}\text { Prometryn } \\
\text { (04036) }\end{array}$ & $\begin{array}{l}\text { Simazine }^{1} \\
\text { (04035) }\end{array}$ & $\begin{array}{l}\text { Tebu- } \\
\text { thiuron' } \\
(82670)\end{array}$ & $\begin{array}{l}\text { Diazinon-d10, } \\
\text { surrogate } \\
\text { (99994) } \\
\text { [percent] }\end{array}$ & $\begin{array}{c}\alpha-\mathrm{HCH}-d 6 \text {, } \\
\text { surrogate } \\
\text { (99995) } \\
\text { [percent] }\end{array}$ \\
\hline [LRL] & & & {$[0.005]$} & {$[0.005]$} & {$[0.02]$} & & \\
\hline \multirow{5}{*}{$\begin{array}{l}\text { Sweetwater Reservoir near pump tower } \\
\quad \text { (SWR01) }\end{array}$} & $02 / 14 / 2003$ & 1130 & - & 0.006 & - & 108 & 100 \\
\hline & 04/08/2003 & 1100 & - & 0.007 & E.01 & 102 & 98.2 \\
\hline & $06 / 17 / 2003$ & 1130 & - & 0.010 & - & 110 & 95.1 \\
\hline & 08/19/2003 & 1130 & - & 0.008 & E.01 & 106 & 83.2 \\
\hline & 08/19/2003 & 1150 & - & 0.008 & - & 104 & 84.4 \\
\hline \multirow{5}{*}{$\begin{array}{l}\text { Sweetwater Reservoir center of minimum } \\
\text { pool (SWR03) }\end{array}$} & 02/14/2003 & 1220 & - & 0.006 & - & 105 & 102 \\
\hline & 04/08/2003 & 1200 & - & 0.006 & - & 99.2 & 98.4 \\
\hline & $06 / 17 / 2003$ & 1210 & - & - & - & 106 & 97.2 \\
\hline & 08/19/2003 & 1230 & - & 0.008 & E.01 & 105 & 90.4 \\
\hline & 08/19/2003 & 1250 & E0.003 & 0.008 & E.01 & 111 & 89.8 \\
\hline \multirow{3}{*}{$\begin{array}{l}\text { Sweetwater Reservoir east end reservoir fill } \\
\text { boundary (SWR06) }\end{array}$} & 02/14/2003 & 1240 & - & 0.006 & E.04 & 111 & 102 \\
\hline & $04 / 08 / 2003$ & 1240 & - & 0.006 & - & 103 & 93.2 \\
\hline & 08/19/2003 & 1320 & - & - & E.01 & 107 & 88.7 \\
\hline \multirow[t]{5}{*}{ Loveland reservoir near dam (LLR01) } & $04 / 9 / 2003$ & 1200 & - & E0.005 & - & 97.4 & 91.4 \\
\hline & $04 / 9 / 2003$ & 1220 & - & 0.006 & - & 102 & 100 \\
\hline & $06 / 18 / 2003$ & 1040 & - & - & - & 106 & 98.1 \\
\hline & 08/20/2003 & 1050 & - & 0.006 & - & 100 & 83.5 \\
\hline & $08 / 20 / 2003$ & 1100 & - & - & - & 112 & 94.9 \\
\hline \multirow{3}{*}{$\begin{array}{l}\text { Sweetwater River at low-flow diversion dam } \\
\text { above SWR (LFDD) }\end{array}$} & $04 / 09 / 2003$ & 1530 & - & E0.005 & 0.02 & 93.4 & 83.8 \\
\hline & $06 / 18 / 2003$ & 1400 & - & - & - & 110 & 104 \\
\hline & $08 / 20 / 2003$ & 1400 & - & - & E.01 & 107 & 92.5 \\
\hline \multirow{3}{*}{$\begin{array}{l}\text { Perdue Treatment Plant—finished water at } \\
\text { SWR (SWR08) }\end{array}$} & 04/09/2003 & 0840 & - & 0.013 & - & 106 & 105 \\
\hline & $06 / 17 / 2003$ & 1550 & - & 0.010 & - & 101 & 98.2 \\
\hline & 08/20/2003 & 1430 & - & 0.007 & E.01 & 100 & 88.3 \\
\hline $\begin{array}{l}\text { Perdue Treatment Plant-imported raw } \\
\text { water at SWR (SWR09) }\end{array}$ & $04 / 09 / 2003$ & 0820 & E0.005 & 0.011 & - & 105 & 94.5 \\
\hline
\end{tabular}

\footnotetext{
${ }^{1}$ National Water Quality Laboratory schedule 2001 is the preferred method.
} 
Table 8A. Pesticide compounds analyzed in filtered-water samples, using U.S. Geological Survey's National Water Quality Laboratory schedule 2060, from the Sweetwater Reservoir watershed, San Diego County, California.

[The parameter code is used in the U.S. Geological Survey computerized data system (National Water Information System) to uniquely identify a specific constituent or property. Laboratory reporting level (LRL) values are reported as micrograms per liter unless noted]

\begin{tabular}{|c|c|c|c|c|c|}
\hline Compound & Parameter code & LRL & Compound & Parameter code & LRL \\
\hline Acifluorfen & 49315 & 0.007 & Fluometuron & 38811 & 0.03 \\
\hline Aldicarb & 49312 & 0.04 & Hydroxyatrazine & 50355 & 0.008 \\
\hline Aldicarb sulfone & 49313 & 0.02 & 3-Hydroxycarbofuran & 49308 & 0.006 \\
\hline Atrazine & 39632 & 0.009 & Imazethapyr & 50407 & 0.02 \\
\hline Bendiocarb & 50299 & 0.03 & Imidacloprid & 61695 & 0.007 \\
\hline Benomyl & 50300 & 0.004 & Linuron & 38478 & 0.01 \\
\hline Bentazon & 38711 & 0.01 & МСРВ & 38487 & 0.01 \\
\hline Bromacil & 04029 & 0.03 & Metalaxyl & 50359 & 0.02 \\
\hline Bromoxynil & 49311 & 0.02 & Methiocarb & 38501 & 0.008 \\
\hline Caffeine & 50305 & 0.50 & Methomyl & 49296 & 0.004 \\
\hline Carbaryl & 49310 & 0.03 & Metsulfuron-methyl & 61697 & 0.03 \\
\hline Carbofuran & 49309 & 0.00 & Neburon & 49294 & 0.01 \\
\hline Cycloate & 04031 & 0.01 & Picloram & 49291 & 0.02 \\
\hline 2,4-D and 2,4-methyl ester & 66469 & 0.009 & Propham & 49236 & 0.010 \\
\hline $2,4-\mathrm{DB}$ & 38746 & 0.02 & Propiconazole & 50471 & 0.02 \\
\hline Dacthal monoacid & 49304 & 0.01 & Propoxur & 38538 & 0.008 \\
\hline Deethylatrazine & 04040 & 0.006 & Siduron & 38548 & 0.02 \\
\hline Deisopropylatrazine & 04038 & 0.04 & Sulfometuron-methyl & 50377 & 0.009 \\
\hline Dicamba & 38442 & 0.01 & Tebuthiuron & 82670 & 0.02 \\
\hline Dichlorprop & 49302 & 0.01 & Terbacil & 04032 & 0.010 \\
\hline Dinoseb & 49301 & 0.01 & Triclopyr & 49235 & 0.02 \\
\hline Diphenamid & 04033 & 0.03 & Barban, surrogate & 90640 & percent \\
\hline Diuron & 49300 & 0.01 & Caffeine-C13, surrogate & 99959 & percent \\
\hline
\end{tabular}

Flumetsulam

0.01 
Table 8B. Analytical results for pesticide compounds detected in filtered-water samples, using U.S. Geological Survey's National Water Quality Laboratory Schedule 2060, from the Sweetwater Reservoir watershed, San Diego County, California.

[See table 1 for site identification number. Time is denoted in 24-hour scale. The five digit number in parentheses below each compound name, the parameter code, is used in the U.S. Geological Survey computerized data system (National Water Information System) to uniquely identify a specific constituent or property. The compounds 2,4-D and 2,4-D methyl ester were summed on a molar basis and reported as 2,4-D. National Water Quality Laboratory schedule 2003 is the preferred method for tebuthiuron. LRL, laboratory reporting level; - , compound not detected at a concentration above laboratory reporting level; E, estimated value; na, not applicable; concentrations reported as micrograms per liter unless noted]

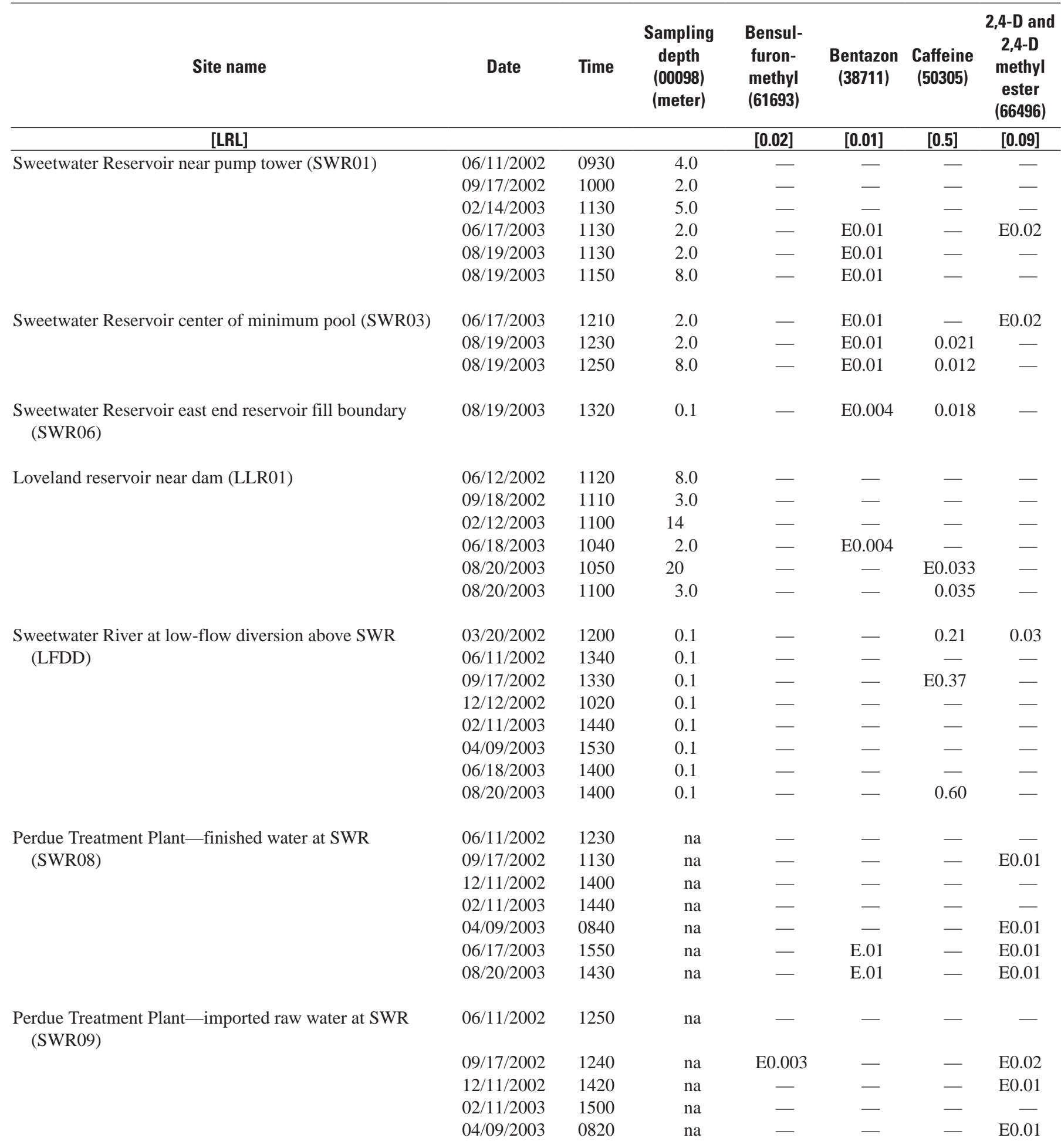


Table 8B. Analytical results for pesticide compounds detected in filtered-water samples, using U.S. Geological Survey's National Water Quality Laboratory Schedule 2060, from the Sweetwater Reservoir watershed, San Diego County, California.-Continued

[See table 1 for site identification number. Time is denoted in 24-hour scale. The five digit number in parentheses below each compound name, the parameter code, is used in the U.S. Geological Survey computerized data system (National Water Information System) to uniquely identify a specific constituent or property. The compounds 2,4-D and 2,4-D methyl ester were summed on a molar basis and reported as 2,4-D. National Water Quality Laboratory schedule 2003 is the preferred method for tebuthiuron. LRL, laboratory reporting level; — , compound not detected at a concentration above laboratory reporting level; E, estimated value; na, not applicable; concentrations reported as micrograms per liter unless noted]

\begin{tabular}{|c|c|c|c|c|c|c|c|}
\hline Site name & Date & Time & $\begin{array}{l}\text { Diphen- } \\
\text { amid } \\
\text { (04033) }\end{array}$ & $\begin{array}{l}\text { Diuron } \\
(49300)\end{array}$ & $\begin{array}{l}\text { Fluo- } \\
\text { meturon } \\
\text { (38811) }\end{array}$ & $\begin{array}{l}\text { Hydroxy- } \\
\text { atrazine } \\
(50355)\end{array}$ & $\begin{array}{l}\text { Ima- } \\
\text { zethapyr } \\
\text { (50407) }\end{array}$ \\
\hline [LRL] & & & {$[0.03]$} & {$[0.01]$} & {$[0.03]$} & {$[0.008]$} & {$[0.02]$} \\
\hline \multirow[t]{6}{*}{ Sweetwater Reservoir near pump tower (SWR01) } & $06 / 11 / 2002$ & 0930 & - & 0.04 & - & - & - \\
\hline & $09 / 17 / 2002$ & 1000 & - & 0.03 & - & $\mathrm{E} 0.008$ & - \\
\hline & $02 / 14 / 2003$ & 1130 & - & 0.02 & - & E.005 & - \\
\hline & $06 / 17 / 2003$ & 1130 & - & 0.04 & - & - & - \\
\hline & 08/19/2003 & 1130 & - & E0.03 & - & E.010 & - \\
\hline & 08/19/2003 & 1150 & - & E0.04 & - & E.009 & - \\
\hline \multirow[t]{3}{*}{ Sweetwater Reservoir center of minimum pool (SWR03) } & $06 / 17 / 2003$ & 1210 & - & $\mathrm{E} 0.05$ & - & - & - \\
\hline & $08 / 19 / 2003$ & 1230 & - & 0.03 & - & - & - \\
\hline & $08 / 19 / 2003$ & 1250 & - & 0.04 & - & E.008 & - \\
\hline $\begin{array}{l}\text { Sweetwater Reservoir east end reservoir fill boundary } \\
\quad \text { (SWR06) }\end{array}$ & 08/19/2003 & 1320 & - & E0.03 & - & E0.007 & - \\
\hline \multirow[t]{6}{*}{ Loveland reservoir near dam (LLR01) } & $06 / 12 / 2002$ & 1120 & - & 0.03 & - & - & - \\
\hline & 09/18/2002 & 1110 & - & 0.02 & - & - & - \\
\hline & $02 / 12 / 2003$ & 1100 & - & 0.02 & - & - & - \\
\hline & $06 / 18 / 2003$ & 1040 & - & 0.02 & - & - & - \\
\hline & 08/20/2003 & 1050 & - & E0.02 & E0.01 & - & - \\
\hline & $08 / 20 / 2003$ & 1100 & - & - & $\mathrm{E} 0.01$ & - & - \\
\hline \multirow{8}{*}{$\begin{array}{l}\text { Sweetwater River at low-flow diversion above SWR } \\
\text { (LFDD) }\end{array}$} & 03/20/2002 & 1200 & E0.02 & 0.32 & - & - & - \\
\hline & $06 / 11 / 2002$ & 1340 & - & 0.02 & - & - & - \\
\hline & 09/17/2002 & 1330 & - & - & - & E0.014 & - \\
\hline & $12 / 12 / 2002$ & 1020 & E0.005 & - & - & - & - \\
\hline & $02 / 11 / 2003$ & 1440 & E0.005 & - & - & - & - \\
\hline & 04/09/2003 & 1530 & E0.004 & - & - & - & - \\
\hline & $06 / 18 / 2003$ & 1400 & E0.003 & $\mathrm{E} 0.02$ & - & - & - \\
\hline & 08/20/2003 & 1400 & E0.005 & - & - & - & E0.01 \\
\hline \multirow{7}{*}{$\begin{array}{l}\text { Perdue Treatment Plant_-finished water at SWR } \\
\quad(\text { SWR08) }\end{array}$} & 06/11/2002 & 1230 & - & 0.03 & - & - & - \\
\hline & $09 / 17 / 2002$ & 1130 & - & - & - & - & - \\
\hline & $12 / 11 / 2002$ & 1400 & - & - & - & - & - \\
\hline & $02 / 11 / 2003$ & 1440 & - & 0.03 & - & E0.007 & - \\
\hline & 04/09/2003 & 0840 & - & E0.12 & - & - & - \\
\hline & $06 / 17 / 2003$ & 1550 & - & E0.04 & - & E0.006 & - \\
\hline & 08/20/2003 & 1430 & - & E0.02 & - & E0.011 & - \\
\hline \multirow{5}{*}{$\begin{array}{l}\text { Perdue Treatment Plant_-imported raw water at SWR } \\
\text { (SWR09) }\end{array}$} & $06 / 11 / 2002$ & 1250 & - & 0.06 & - & - & - \\
\hline & 09/17/2002 & 1240 & - & 0.02 & - & E0.006 & - \\
\hline & $12 / 11 / 2002$ & 1420 & - & 0.02 & - & - & - \\
\hline & $02 / 11 / 2003$ & 1500 & - & 0.04 & - & E0.005 & - \\
\hline & $04 / 09 / 2003$ & 0820 & - & 0.14 & - & - & - \\
\hline
\end{tabular}


Table 8B. Analytical results for pesticide compounds detected in filtered-water samples, using U.S. Geological Survey's National Water Quality Laboratory Schedule 2060, from the Sweetwater Reservoir watershed, San Diego County, California.—Continued

[See table 1 for site identification number. Time is denoted in 24-hour scale. The five digit number in parentheses below each compound name, the parameter code, is used in the U.S. Geological Survey computerized data system (National Water Information System) to uniquely identify a specific constituent or property. The compounds 2,4-D and 2,4-D methyl ester were summed on a molar basis and reported as 2,4-D. National Water Quality Laboratory schedule 2003 is the preferred method for tebuthiuron. LRL, laboratory reporting level; - , compound not detected at a concentration above laboratory reporting level; E, estimated value; na, not applicable; concentrations reported as micrograms per liter unless noted]

\begin{tabular}{|c|c|c|c|c|c|c|}
\hline Site name & Date & Time & $\begin{array}{l}\text { Linuron } \\
(38478)\end{array}$ & $\begin{array}{l}\text { Siduron } \\
\text { (38548) }\end{array}$ & $\begin{array}{c}\text { Sulfo- } \\
\text { meturon- } \\
\text { methyl } \\
(50337)\end{array}$ & $\begin{array}{l}\text { Tebu- } \\
\text { thiuron } \\
\text { (82670) }\end{array}$ \\
\hline [LRL] & & & {$[0.01]$} & {$[0.02]$} & {$[0.009]$} & {$[0.02]$} \\
\hline \multirow[t]{6}{*}{ Sweetwater Reservoir near pump tower (SWR01) } & $06 / 11 / 2002$ & 0930 & - & - & - & - \\
\hline & 09/17/2002 & 1000 & - & - & - & - \\
\hline & $02 / 14 / 2003$ & 1130 & - & - & - & - \\
\hline & $06 / 17 / 2003$ & 1130 & - & - & - & - \\
\hline & $08 / 19 / 2003$ & 1130 & - & - & - & - \\
\hline & $08 / 19 / 2003$ & 1150 & - & - & - & - \\
\hline \multirow[t]{3}{*}{ Sweetwater Reservoir center of minimum pool (SWR03) } & $06 / 17 / 2003$ & 1210 & - & - & - & - \\
\hline & 08/19/2003 & 1230 & - & - & - & - \\
\hline & 08/19/2003 & 1250 & - & - & - & - \\
\hline Sweetwater Reservoir east end reservoir fill boundary (SWR06) & $08 / 19 / 2003$ & 1320 & - & - & - & - \\
\hline \multirow[t]{6}{*}{ Loveland reservoir near dam (LLR01) } & $06 / 12 / 2002$ & 1120 & - & - & - & - \\
\hline & $09 / 18 / 2002$ & 1110 & - & - & - & - \\
\hline & $02 / 12 / 2003$ & 1100 & - & - & - & - \\
\hline & 06/18/2003 & 1040 & - & - & - & - \\
\hline & 08/20/2003 & 1050 & - & - & - & - \\
\hline & 08/20/2003 & 1100 & - & - & - & - \\
\hline \multirow{8}{*}{$\begin{array}{l}\text { Sweetwater River at low-flow diversion above SWR } \\
\text { (LFDD) }\end{array}$} & 03/20/2002 & 1200 & - & - & E0.034 & 0.009 \\
\hline & $06 / 11 / 2002$ & 1340 & - & - & - & - \\
\hline & 09/17/2002 & 1330 & - & - & - & E0.003 \\
\hline & $12 / 12 / 2002$ & 1020 & - & - & - & E0.006 \\
\hline & $02 / 11 / 2003$ & 1440 & - & E0.01 & E0.004 & E0.005 \\
\hline & 04/09/2003 & 1530 & - & - & - & - \\
\hline & 06/18/2003 & 1400 & - & - & - & - \\
\hline & 08/20/2003 & 1400 & - & - & - & E0.005 \\
\hline \multirow[t]{7}{*}{ Perdue Treatment Plant—finished water at SWR (SWR08) } & $06 / 11 / 2002$ & 1230 & - & - & - & - \\
\hline & 09/17/2002 & 1130 & - & - & - & - \\
\hline & $12 / 11 / 2002$ & 1400 & E0.01 & - & - & - \\
\hline & $02 / 11 / 2003$ & 1440 & - & - & - & - \\
\hline & 04/09/2003 & 0840 & - & - & - & - \\
\hline & 06/17/2003 & 1550 & - & - & - & - \\
\hline & 08/20/2003 & 1430 & - & - & - & E0.01 \\
\hline \multirow[t]{5}{*}{ Perdue Treatment Plant-imported raw water at SWR (SWR09) } & $06 / 11 / 2002$ & 1250 & - & - & - & - \\
\hline & 09/17/2002 & 1240 & - & - & - & - \\
\hline & $12 / 11 / 2002$ & 1420 & - & - & - & - \\
\hline & $02 / 11 / 2003$ & 1500 & & & & \\
\hline & 04/09/2003 & 0820 & & & & \\
\hline
\end{tabular}


Table 8B. Analytical results for pesticide compounds detected in filtered-water samples, using U.S. Geological Survey's National Water Quality Laboratory Schedule 2060, from the Sweetwater Reservoir watershed, San Diego County, California.Continued

[See table 1 for site identification number. Time is denoted in 24-hour scale. The five digit number in parentheses below each compound name, the parameter code, is used in the U.S. Geological Survey computerized data system (National Water Information System) to uniquely identify a specific constituent or property. The compounds 2,4-D and 2,4-D methyl ester were summed on a molar basis and reported as 2,4-D. National Water Quality Laboratory schedule 2003 is the preferred method for tebuthiuron. LRL, laboratory reporting level; - , compound not detected at a concentration above laboratory reporting level; E, estimated value; na, not applicable; concentrations reported as micrograms per liter unless noted]

\begin{tabular}{|c|c|c|c|c|c|}
\hline Site name & Date & Time & $\begin{array}{c}\text { Barban, } \\
\text { surrogate } \\
(90640) \\
\text { (percent) }\end{array}$ & $\begin{array}{l}\text { Caffeine-C13, } \\
\text { surrogate } \\
\text { (99959) } \\
\text { (percent) }\end{array}$ & $\begin{array}{c}\text { 2,4,5-T, } \\
\text { surrogate } \\
\text { (99958) } \\
\text { (percent) }\end{array}$ \\
\hline \multicolumn{6}{|l|}{ [LRL] } \\
\hline \multirow[t]{6}{*}{ Sweetwater Reservoir near pump tower (SWR01) } & $06 / 11 / 2002$ & 0930 & 76.5 & 76.6 & 77.2 \\
\hline & $09 / 17 / 2002$ & 1000 & E174 & E148 & 72.0 \\
\hline & $02 / 14 / 2003$ & 1130 & 145 & 69.4 & 77.7 \\
\hline & $06 / 17 / 2003$ & 1130 & 44.0 & 63.7 & 64.7 \\
\hline & $08 / 19 / 2003$ & 1130 & 102 & 75.7 & 99.1 \\
\hline & $08 / 19 / 2003$ & 1150 & 66.0 & 78.6 & 96.6 \\
\hline \multirow[t]{3}{*}{ Sweetwater Reservoir center of minimum pool (SWR03) } & $06 / 17 / 2003$ & 1210 & 123 & 80.7 & 87.8 \\
\hline & $08 / 19 / 2003$ & 1230 & 79.1 & 70.0 & 95.5 \\
\hline & $08 / 19 / 2003$ & 1250 & 75.8 & 65.5 & 86.8 \\
\hline $\begin{array}{l}\text { Sweetwater Reservoir east end reservoir fill boundary } \\
\quad \text { (SWR06) }\end{array}$ & $08 / 19 / 2003$ & 1320 & 64.7 & 74.8 & 90.6 \\
\hline \multirow[t]{6}{*}{ Loveland reservoir near dam (LLR01) } & $06 / 12 / 2002$ & 1120 & 102 & 74.9 & 95.9 \\
\hline & $09 / 18 / 2002$ & 1110 & 75.4 & 155 & 87.4 \\
\hline & $02 / 12 / 2003$ & 1100 & 128 & 77.3 & 70.8 \\
\hline & $06 / 18 / 2003$ & 1040 & 55.3 & 69.2 & 61.1 \\
\hline & $08 / 20 / 2003$ & 1050 & E45.8 & E89.1 & 85.3 \\
\hline & $08 / 20 / 2003$ & 1100 & 65.0 & 78.3 & 92.9 \\
\hline \multirow[t]{8}{*}{ Sweetwater River at low-flow diversion above SWR (LFDD) } & 03/20/2002 & 1200 & 42.0 & 73.7 & 62.8 \\
\hline & $06 / 11 / 2002$ & 1340 & 101 & 76.9 & 73.9 \\
\hline & $09 / 17 / 2002$ & 1330 & E91.9 & E172 & 73.6 \\
\hline & $12 / 12 / 2002$ & 1020 & 69.5 & 125 & 76.6 \\
\hline & $02 / 11 / 2003$ & 1440 & 96.2 & 82.7 & 61.8 \\
\hline & $04 / 09 / 2003$ & 1530 & 47.5 & 58.9 & 68.9 \\
\hline & $06 / 18 / 2003$ & 1400 & 72.6 & 84.1 & 78.9 \\
\hline & $08 / 20 / 2003$ & 1400 & 61.8 & 99.9 & 92.0 \\
\hline \multirow[t]{7}{*}{ Perdue Treatment Plant-finished water at SWR (SWR08) } & $06 / 11 / 2002$ & 1230 & 94.2 & 71.7 & 84.4 \\
\hline & $09 / 17 / 2002$ & 1130 & E113 & 117 & 97.7 \\
\hline & $12 / 11 / 2002$ & 1400 & 69.0 & 111 & 75.8 \\
\hline & $02 / 11 / 2003$ & 1440 & E200 & 66.9 & 77.2 \\
\hline & $04 / 09 / 2003$ & 0840 & 117 & 48.9 & 76.4 \\
\hline & $06 / 17 / 2003$ & 1550 & 121 & 75.8 & 89.4 \\
\hline & $08 / 20 / 2003$ & 1430 & 111 & 68.0 & 96.3 \\
\hline \multirow[t]{5}{*}{ Perdue Treatment Plant—imported raw water at SWR (SWR09) } & $06 / 11 / 2002$ & 1250 & 113 & 80.3 & 68.3 \\
\hline & 09/17/2002 & 1240 & E74.7 & 116 & 92.5 \\
\hline & $12 / 11 / 2002$ & 1420 & 110 & 110 & 81.6 \\
\hline & $02 / 11 / 2003$ & 1500 & 117 & 59.4 & 78.5 \\
\hline & $04 / 09 / 2003$ & 0820 & 86.8 & 47.8 & 69.4 \\
\hline
\end{tabular}


Table 9A. Analytical results for volatile organic compounds in air with low breakthrough volumes from the Sweetwater Reservoir atmospheric site, San Diego County, California.

[The site identification number is 324141117001601. Concentrations are given in parts per billion by volume (pbbv). mm of $\mathrm{Hg}$, millimeters of mercury; NA, not analyzed; E, estimated value. —, compound was not detected at a concentration above the laboratory reporting level. *, suspect because concentration in laboratory blank was greater than 10 percent of that in environmental sample]

\begin{tabular}{|c|c|c|c|c|c|c|c|c|}
\hline $\begin{array}{c}\text { Date } \\
\text { (mm/dd/yyyy) }\end{array}$ & $\begin{array}{l}\text { Pressure } \\
(\mathrm{mm} \text { of } \mathrm{Hg})\end{array}$ & $\begin{array}{c}\text { Temper- } \\
\text { ature } \\
{ }^{\circ} \mathrm{C}\end{array}$ & $\begin{array}{l}\text { Dichlorodifluoro- } \\
\text { methane } \\
\text { (CFC-12) }\end{array}$ & $\begin{array}{l}\text { Chloro- } \\
\text { methane }\end{array}$ & $\begin{array}{l}\text { Chloro- } \\
\text { ethene } \\
\text { (vinyl } \\
\text { chloride) }\end{array}$ & $\begin{array}{l}\text { Bromo- } \\
\text { methane }\end{array}$ & $\begin{array}{l}\text { Chloro- } \\
\text { ethane }\end{array}$ & $\begin{array}{c}\text { Bromo- } \\
\text { ethene } \\
\text { (vinyl } \\
\text { bromide) }\end{array}$ \\
\hline $10 / 10 / 2001$ & 754 & 18.8 & 0.72 & - & - & - & - & - \\
\hline $10 / 22 / 2001$ & 753 & 17.8 & 0.45 & - & - & - & - & - \\
\hline $11 / 15 / 2001$ & 753 & 15.7 & 0.90 & - & - & - & - & - \\
\hline $11 / 27 / 2001$ & 755 & 11.2 & 0.28 & - & - & - & - & - \\
\hline $12 / 09 / 2001$ & 752 & 12.3 & 0.25 & - & - & - & - & - \\
\hline $12 / 21 / 2001$ & 758 & 11.4 & 0.58 & - & - & - & - & - \\
\hline 01/03/2002 & 757 & 11.9 & NA & NA & NA & NA & NA & NA \\
\hline 01/14/2002 & 753 & 10.6 & NA & NA & NA & NA & NA & NA \\
\hline $01 / 28 / 2002$ & 754 & 10.6 & - & - & - & - & - & - \\
\hline 02/07/2002 & 761 & 10.2 & 0.78 & - & - & - & - & - \\
\hline 02/19/2002 & 760 & 10.9 & 0.84 & - & - & - & - & - \\
\hline 03/03/2002 & 761 & 13.9 & 2.11 & - & - & - & - & - \\
\hline $03 / 27 / 2002$ & 754 & 12.5 & $0.61 *$ & - & - & - & - & - \\
\hline 04/20/2002 & 761 & 13.9 & 0.87 & - & - & - & - & - \\
\hline 05/14/2002 & 751 & 17.0 & 0.29 & - & - & E2.08 & - & - \\
\hline $05 / 26 / 2002$ & 753 & 13.4 & 0.60 & - & - & - & - & - \\
\hline 06/07/2002 & 750 & 16.2 & 0.35 & - & - & - & - & - \\
\hline 06/19/2002 & 750 & 16.2 & 0.74 & - & - & - & - & - \\
\hline 07/01/2002 & 753 & 16.9 & 0.76 & - & - & - & - & - \\
\hline 07/13/2002 & 751 & 18.1 & 0.48 & - & - & - & - & - \\
\hline $07 / 25 / 2002$ & 754 & 18.6 & 0.55 & - & - & - & - & - \\
\hline 08/06/2002 & 753 & 17.2 & 0.72 & - & - & - & - & - \\
\hline 08/18/2002 & 752 & 18.5 & 0.38 & - & - & - & - & - \\
\hline 08/30/2002 & 751 & 20.8 & 0.73 & - & - & - & - & - \\
\hline 09/11/2002 & 750 & 21.8 & 0.74 & - & - & - & - & - \\
\hline 09/23/2002 & 749 & 22.7 & 0.71 & - & - & - & - & - \\
\hline $10 / 05 / 2002$ & 752 & 19.0 & 0.97 & - & - & - & - & - \\
\hline $10 / 17 / 2002$ & 752 & 16.2 & 0.50 & - & - & - & - & - \\
\hline $10 / 29 / 2002$ & 752 & 15.8 & 0.37 & - & - & - & - & - \\
\hline $11 / 10 / 2002$ & 756 & 16.2 & 0.56 & - & - & - & - & - \\
\hline $11 / 22 / 2002$ & 754 & 17.0 & 0.38 & - & - & - & - & - \\
\hline $12 / 04 / 2002$ & 758 & 13.6 & 0.72 & - & - & - & - & - \\
\hline $01 / 20 / 2003$ & 758 & 14.5 & 0.71 & - & - & E0.87 & - & 一 \\
\hline 02/04/2003 & 754 & 13.8 & 0.54 & - & - & - & - & - \\
\hline $02 / 14 / 2003$ & 753 & 14.9 & - & - & - & - & - & - \\
\hline
\end{tabular}


Table 9A. Analytical results for volatile organic compounds in air with low breakthrough volumes from the Sweetwater Reservoir atmospheric site, San Diego County, California.-Continued

[The site identification number is 324141117001601 . Concentrations are given in parts per billion by volume (ppbv). mm of Hg, millimeters of mercury; NA, not analyzed; E, estimated value. - , compound was not detected at a concentration above the laboratory reporting level. *, suspect because concentration in laboratory blank is greater than 10 percent of that in environmental sample]

\begin{tabular}{ccccccccc}
\hline $\begin{array}{c}\text { Date } \\
\text { (mm/dd/yyyy) }\end{array}$ & $\begin{array}{c}\text { Pressure } \\
\text { (mm of Hg) }\end{array}$ & $\begin{array}{c}\text { Temper- } \\
\text { ature } \\
{ }^{\circ} \mathbf{C}\end{array}$ & $\begin{array}{c}\text { Dichlorodifluoro- } \\
\text { methane } \\
\text { (CFC-12) }\end{array}$ & $\begin{array}{c}\text { Chloro- } \\
\text { methane }\end{array}$ & $\begin{array}{c}\text { Chloro- } \\
\text { ethene } \\
\text { (vinyl } \\
\text { chloride) }\end{array}$ & $\begin{array}{c}\text { Bromo- } \\
\text { methane }\end{array}$ & $\begin{array}{c}\text { Chloro- } \\
\text { ethane }\end{array}$ & $\begin{array}{c}\text { Bromo- } \\
\text { ethene } \\
\text { (vinyl } \\
\text { bromide) }\end{array}$ \\
\hline $02 / 26 / 2003$ & 752 & 12.6 & 0.60 & - & - & - & - & - \\
$03 / 10 / 2003$ & 753 & 15.0 & 1.81 & - & - & E0.31 & - & - \\
$03 / 22 / 2003$ & 756 & 15.4 & 0.74 & - & - & - & - & - \\
$04 / 03 / 2003$ & 757 & 12.4 & 0.63 & - & - & - & - & - \\
$04 / 15 / 2003$ & 757 & 12.6 & 0.48 & - & - & - & - & - \\
$05 / 09 / 2003$ & 762 & 13.8 & 0.68 & - & - & - & - & - \\
$05 / 21 / 2003$ & 758 & 20.2 & 0.41 & - & - & - & - & - \\
$06 / 02 / 2003$ & 758 & 16.4 & 0.56 & - & - & - & - & - \\
$06 / 14 / 2003$ & 759 & 19.1 & 0.78 & - & - & E0.40 & - & - \\
$06 / 26 / 2003$ & 759 & 19.2 & 0.72 & - & - & E0.20 & - & - \\
$07 / 09 / 2003$ & 758 & 18.7 & 0.72 & - & - & - & - & - \\
$07 / 20 / 2003$ & 761 & 22.9 & 0.72 & - & - & - & - & - \\
$08 / 01 / 2003$ & 762 & 23.0 & 0.72 & - & - & - & - & - \\
$08 / 13 / 2003$ & 758 & 23.6 & 0.66 & - & - & - & - & - \\
$08 / 25 / 2003$ & 759 & 23.0 & 0.59 & - & - & - & - & - \\
$09 / 06 / 2003$ & 759 & 24.4 & 0.47 & - & - & - & - & - \\
$09 / 18 / 2003$ & 757 & 19.5 & 0.42 & - & - & - & - & - \\
\hline
\end{tabular}


.Table 9B. Analytical results for volatile organic compounds in air with high breakthrough volumes from the Sweetwater Reservoir atmospheric site, San Diego County, California.

[The site identification number is 324141117001601 . Concentrations are given in part per billion by volume (ppbv). E, estimated value; NA, not analyzed; - , compound not detected at a concentration above the laboratory reporting level]

\begin{tabular}{|c|c|c|c|c|c|c|c|c|c|}
\hline $\begin{array}{c}\text { Date } \\
\text { (mm/dd/yyyy) }\end{array}$ & $\begin{array}{l}\text { Dibromo- } \\
\text { methane }\end{array}$ & $\begin{array}{l}\text { Bromo- } \\
\text { dichloro- } \\
\text { methane }\end{array}$ & $\begin{array}{l}\text { Carbon } \\
\text { tetra- } \\
\text { chloride }\end{array}$ & $\begin{array}{l}\text { 1,2-Dichloro- } \\
\text { ethane }\end{array}$ & Bromoform & $\begin{array}{c}\text { Dibromo- } \\
\text { chloro- } \\
\text { methane }\end{array}$ & Chloroform & Toluene & Benzene \\
\hline $10 / 10 / 2001$ & NA & NA & NA & NA & $\mathrm{NA}$ & NA & NA & NA & NA \\
\hline $10 / 22 / 2001$ & NA & NA & NA & NA & NA & NA & NA & NA & NA \\
\hline $11 / 15 / 2001$ & - & - & E0.03 & - & - & - & E0.02 & 0.99 & 0.20 \\
\hline $11 / 27 / 2001$ & - & - & E0.14 & - & - & - & E0.04 & 1.10 & 0.36 \\
\hline $12 / 09 / 2001$ & E0.01 & - & E0.12 & E0.01 & - & - & E0.02 & 0.68 & 0.34 \\
\hline $12 / 21 / 2001$ & - & - & 0.02 & - & - & - & - & 0.27 & 0.18 \\
\hline $01 / 03 / 2002$ & - & - & E0.03 & - & - & - & - & E0.08 & 0.37 \\
\hline 01/14/2002 & - & - & E0.22 & E0.02 & - & - & E0.02 & 2.25 & 0.99 \\
\hline $01 / 28 / 2002$ & - & - & E0.08 & - & - & - & - & 0.37 & - \\
\hline 02/07/2002 & - & - & E0.09 & - & - & - & - & 2.13 & 1.42 \\
\hline $02 / 19 / 2002$ & - & - & E0.11 & - & - & - & E0.03 & 0.87 & 0.42 \\
\hline 03/03/2002 & - & - & E0.03 & - & - & - & - & 0.33 & 0.58 \\
\hline $03 / 27 / 2002$ & - & - & E0.12 & - & - & - & - & 0.43 & - \\
\hline $04 / 20 / 2002$ & - & - & E0.07 & - & - & - & E0.02 & 0.45 & - \\
\hline $05 / 14 / 2002$ & - & - & E0.06 & - & - & - & E0.02 & 0.49 & 0.37 \\
\hline $05 / 26 / 2002$ & - & - & E0.09 & - & - & - & E0.01 & 0.42 & - \\
\hline 06/07/2002 & - & - & E0.23 & - & - & - & E0.05 & 0.63 & 0.33 \\
\hline $06 / 19 / 2002$ & - & - & E0.15 & - & - & - & E0.01 & 0.87 & 0.26 \\
\hline $07 / 01 / 2002$ & - & - & 0.36 & - & - & - & E0.08 & 1.10 & 0.58 \\
\hline $07 / 13 / 2002$ & - & - & E0.20 & - & - & - & E0.02 & 0.53 & 0.33 \\
\hline $07 / 25 / 2002$ & - & - & E0.23 & - & - & - & E0.02 & 0.59 & 0.29 \\
\hline 08/06/2002 & - & - & E0.19 & - & - & - & E0.01 & 0.57 & 0.34 \\
\hline 08/18/2002 & - & - & E0.25 & - & - & - & E0.01 & 0.21 & 0.39 \\
\hline 08/30/2002 & - & - & E0.13 & - & - & - & E0.03 & 1.56 & 0.68 \\
\hline $09 / 11 / 2002$ & NA & NA & NA & NA & NA & NA & NA & NA & NA \\
\hline $09 / 23 / 2002$ & NA & NA & NA & NA & NA & NA & NA & NA & NA \\
\hline $10 / 05 / 2002$ & - & - & E0.15 & - & - & - & E0.04 & 0.60 & 0.44 \\
\hline $10 / 17 / 2002$ & - & - & 0.20 & - & - & - & 0.09 & 0.18 & 0.26 \\
\hline $10 / 29 / 2002$ & - & - & 0.28 & - & - & - & E0.08 & 0.46 & 0.36 \\
\hline $11 / 10 / 2002$ & - & - & E0.16 & - & - & - & 0.16 & 0.97 & 0.51 \\
\hline $11 / 22 / 2002$ & - & - & E0.10 & - & - & - & E0.03 & 2.00 & 0.69 \\
\hline $12 / 04 / 2002$ & - & - & E0.03 & - & - & - & - & 0.47 & E0.12 \\
\hline
\end{tabular}


Table 9B. Analytical results for volatile organic compounds in air with high breakthrough volumes from the Sweetwater Reservoir atmospheric site, San Diego County, California.-Continued

[The site identification number is 324141117001601 . Concentrations are given in part per billion by volume (ppbv). E, estimated value; NA, not analyzed; _, compound not detected at a concentration above the laboratory reporting level]

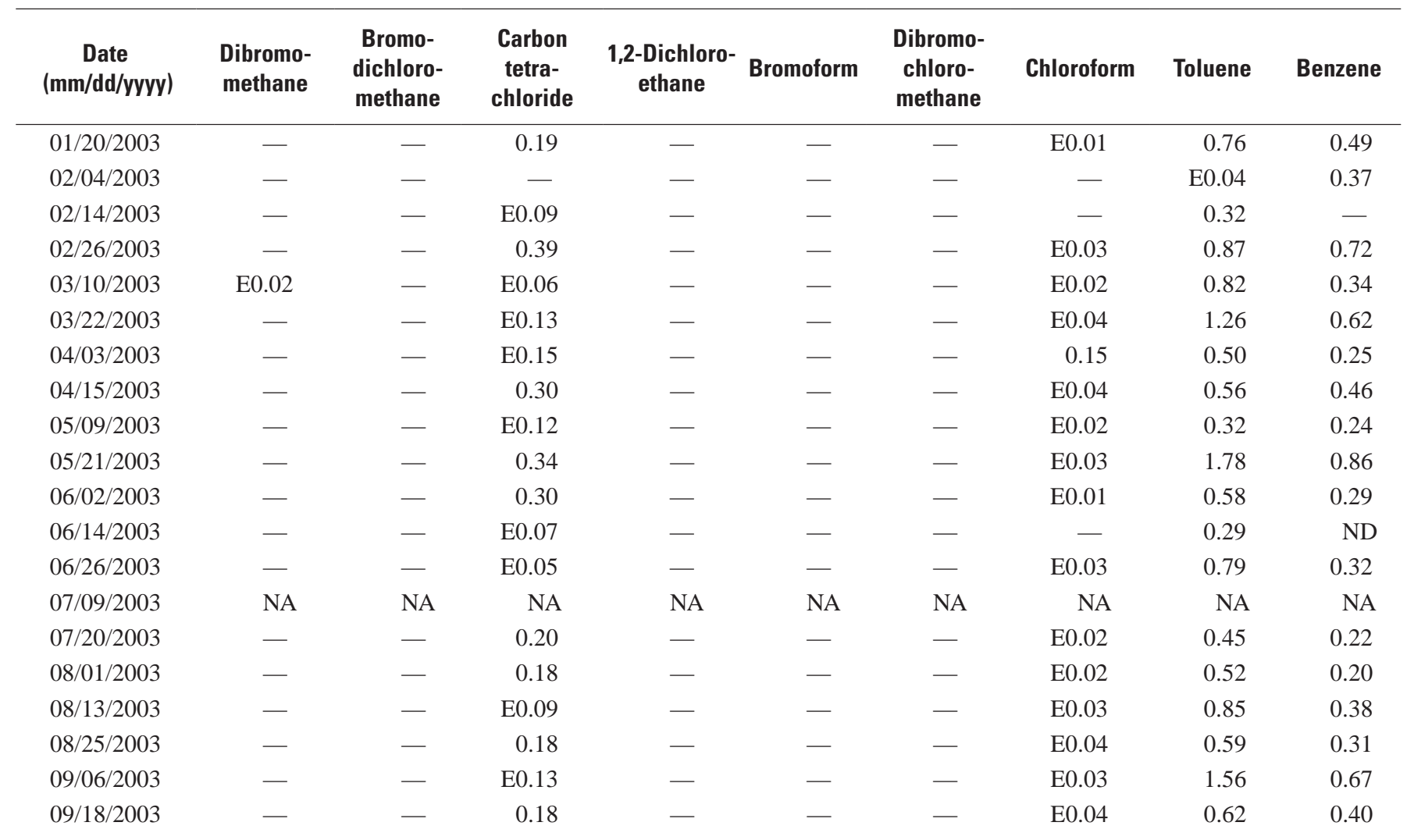


Table 9B. Analytical results for volatile organic compounds in air with high breakthrough volumes from the Sweetwater Reservoir atmospheric site, San Diego County, California.-Continued

[The site identification number is 324141117001601 . Concentrations are given in parts per billion by volume (ppbv). E, estimated value; NA, not analyzed; _, compound not detected at a concentration above the laboratory reporting level]

\begin{tabular}{|c|c|c|c|c|c|c|c|c|c|}
\hline $\begin{array}{c}\text { Date } \\
\text { (mm/dd/yyyy) }\end{array}$ & $\begin{array}{c}\text { 2-Propene- } \\
\text { nitrile } \\
\text { (Acrylonitrile) }\end{array}$ & $\begin{array}{l}\text { Chloro- } \\
\text { benzene }\end{array}$ & $\begin{array}{c}\text { Ethyl- } \\
\text { benzene }\end{array}$ & $\begin{array}{l}\text { Hexachloro- } \\
\text { ethane }\end{array}$ & $\begin{array}{l}\text { Methylene } \\
\text { chloride }\end{array}$ & $\begin{array}{l}\text { Tetrachloro- } \\
\text { ethene } \\
\text { (PCE) }\end{array}$ & $\begin{array}{l}\text { 1,1- Dichloro- } \\
\text { ethane }\end{array}$ & $\begin{array}{l}1,1-\mathrm{Di}- \\
\text { chloro- } \\
\text { ethene }\end{array}$ & $\begin{array}{l}\text { 1,1,1-Tri- } \\
\text { chloro- } \\
\text { ethane }\end{array}$ \\
\hline $10 / 10 / 2001$ & NA & NA & NA & NA & NA & NA & NA & NA & NA \\
\hline $10 / 22 / 2001$ & NA & NA & NA & NA & NA & NA & NA & NA & NA \\
\hline $11 / 15 / 2001$ & - & - & 0.09 & - & E0.07 & E0.03 & - & - & E0.02 \\
\hline $11 / 27 / 2001$ & - & - & 0.12 & - & 0.13 & E0.03 & - & - & E0.05 \\
\hline $12 / 09 / 2001$ & - & - & 0.11 & - & - & - & - & - & E0.04 \\
\hline $12 / 21 / 2001$ & - & E0.01 & E0.02 & - & E0.07 & - & - & - & - \\
\hline $01 / 03 / 2002$ & - & E0.01 & E0.03 & - & E0.04 & - & - & - & E0.01 \\
\hline $01 / 14 / 2002$ & - & - & 0.41 & - & 0.17 & 0.06 & - & - & 0.09 \\
\hline $01 / 28 / 2002$ & - & - & 0.07 & - & - & - & - & - & E0.02 \\
\hline $02 / 07 / 2002$ & - & - & 0.73 & - & E0.10 & E0.06 & - & - & E0.04 \\
\hline 02/19/2002 & - & - & 0.12 & - & E0.08 & E0.03 & - & - & E0.04 \\
\hline 03/03/2002 & E0.04 & E0.01 & E0.03 & - & 0.18 & - & - & - & E0.01 \\
\hline $03 / 27 / 2002$ & - & - & E0.06 & - & - & - & - & - & E0.04 \\
\hline 04/20/2002 & - & - & E0.06 & - & E0.09 & - & - & - & E0.03 \\
\hline $05 / 14 / 2002$ & - & - & E0.05 & - & 0.14 & E0.01 & - & - & E0.03 \\
\hline 05/26/2002 & - & - & E0.05 & - & 0.10 & E0.01 & - & - & E0.04 \\
\hline 06/07/2002 & - & - & 0.13 & - & 0.48 & - & - & - & E0.06 \\
\hline 06/19/2002 & - & - & 0.11 & - & E0.05 & E0.01 & - & - & E0.05 \\
\hline $07 / 01 / 2002$ & - & - & 0.21 & - & 0.24 & E0.04 & - & - & 0.15 \\
\hline $07 / 13 / 2002$ & - & - & 0.14 & - & E0.04 & E0.02 & - & - & E0.06 \\
\hline $07 / 25 / 2002$ & - & - & 0.10 & - & E0.06 & E0.03 & - & - & E0.06 \\
\hline 08/06/2002 & - & - & 0.11 & - & E0.05 & E0.02 & - & - & E0.05 \\
\hline 08/18/2002 & - & - & E0.05 & - & E0.03 & - & - & - & E0.06 \\
\hline 08/30/2002 & - & E0.01 & 0.23 & - & 0.33 & 0.10 & - & - & E0.05 \\
\hline 09/11/2002 & NA & NA & NA & NA & NA & NA & NA & NA & NA \\
\hline 09/23/2002 & NA & NA & NA & NA & NA & NA & NA & NA & NA \\
\hline $10 / 05 / 2002$ & - & - & 0.21 & - & E0.06 & E0.02 & - & - & E0.04 \\
\hline 10/17/2002 & - & - & E0.04 & - & 0.17 & E0.01 & - & - & E0.04 \\
\hline 10/29/2002 & - & - & 0.16 & - & 0.12 & E0.01 & - & - & E0.07 \\
\hline $11 / 10 / 2002$ & - & - & 0.41 & - & 0.26 & - & - & - & 0.08 \\
\hline $11 / 22 / 2002$ & - & - & 0.28 & - & 0.13 & 0.07 & - & - & E0.04 \\
\hline $12 / 04 / 2002$ & - & - & E0.07 & - & E0.06 & E0.02 & - & - & E0.01 \\
\hline 01/20/2003 & - & - & 0.15 & - & E0.03 & E0.03 & - & - & E0.06 \\
\hline $02 / 04 / 2003$ & - & E0.01 & E0.01 & - & E0.02 & E0.01 & - & - & - \\
\hline $02 / 14 / 2003$ & - & - & E0.05 & - & E0.04 & E0.01 & - & - & E0.03 \\
\hline $02 / 26 / 2003$ & - & - & 0.20 & - & E0.07 & E0.02 & - & - & 0.10 \\
\hline $03 / 10 / 2003$ & - & - & 0.11 & - & 0.22 & E0.03 & - & - & E0.02 \\
\hline $03 / 22 / 2003$ & - & - & 0.17 & - & 0.11 & E0.04 & - & - & E0.04 \\
\hline 04/03/2003 & - & E0.01 & 0.08 & - & 0.12 & E0.01 & - & - & E0.06 \\
\hline
\end{tabular}


Table 9B. Analytical results for volatile organic compounds in air with high breakthrough volumes from the Sweetwater Reservoir atmospheric site, San Diego County, California.-Continued

[The site identification number is 324141117001601 . Concentrations are given in parts per billion by volume (ppbv). E, estimated value; NA, not analyzed; _, compound not detected at a concentration above the laboratory reporting level]

\begin{tabular}{|c|c|c|c|c|c|c|c|c|c|}
\hline $\begin{array}{c}\text { Date } \\
\text { (mm/dd/yyyy) }\end{array}$ & $\begin{array}{l}\text { 2-Propene- } \\
\text { nitrile } \\
\text { (Acrylonitrile) }\end{array}$ & $\begin{array}{l}\text { Chloro- } \\
\text { benzene }\end{array}$ & $\begin{array}{c}\text { Ethyl- } \\
\text { benzene }\end{array}$ & $\begin{array}{c}\text { Hexachloro- } \\
\text { ethane }\end{array}$ & $\begin{array}{l}\text { Methylene } \\
\text { chloride }\end{array}$ & $\begin{array}{c}\text { Tetrachloro- } \\
\text { ethene } \\
\text { (PCE) }\end{array}$ & $\begin{array}{l}\text { 1,1- Dichloro- } \\
\text { ethane }\end{array}$ & $\begin{array}{l}\text { 1,1- Di- } \\
\text { chloro- } \\
\text { ethene }\end{array}$ & $\begin{array}{c}\text { 1,1,1-Tri- } \\
\text { chloro- } \\
\text { ethane }\end{array}$ \\
\hline $04 / 15 / 2003$ & - & E0.01 & 0.09 & - & E0.08 & E0.02 & - & - & 0.09 \\
\hline 05/09/2003 & - & E0.01 & E0.04 & - & 0.13 & E0.01 & - & - & E0.03 \\
\hline $05 / 21 / 2003$ & - & E0.01 & 0.31 & - & 0.14 & E0.06 & - & - & 0.10 \\
\hline $06 / 02 / 2003$ & - & - & 0.11 & - & E0.06 & E0.02 & - & - & 0.07 \\
\hline $06 / 14 / 2003$ & - & - & E0.05 & - & E0.09 & E0.01 & - & - & E0.03 \\
\hline $06 / 26 / 2003$ & - & E0.01 & 0.15 & - & E0.10 & E0.02 & - & - & E0.03 \\
\hline 07/09/2003 & NA & NA & NA & NA & $\mathrm{NA}$ & NA & NA & NA & NA \\
\hline 07/20/2003 & - & - & 0.10 & - & E0.04 & E0.01 & - & - & E0.05 \\
\hline 08/01/2003 & - & E0.01 & 0.12 & - & E0.09 & E0.03 & - & 一 & E0.04 \\
\hline 08/13/2003 & - & - & 0.15 & - & E0.06 & E0.02 & - & - & E0.04 \\
\hline $08 / 25 / 2003$ & - & E0.01 & 0.14 & - & E0.06 & E0.03 & - & - & E0.04 \\
\hline 09/06/2003 & - & E0.01 & 0.23 & - & 0.33 & 0.10 & - & - & E0.05 \\
\hline 09/18/2003 & - & - & 0.21 & - & E0.07 & E0.02 & - & - & E0.05 \\
\hline
\end{tabular}


Table 9B. Analytical results for volatile organic compounds in air with high breakthrough volumes from the Sweetwater Reservoir atmospheric site, San Diego County, California.-Continued

[The site identification number is 324141117001601 . Concentrations are given in parts per billion by volume (ppbv). E, estimated value; NA, not analyzed; —, compound not detected at a concentration above the laboratory reporting level]

\begin{tabular}{|c|c|c|c|c|c|c|c|c|c|}
\hline $\begin{array}{c}\text { Date } \\
\text { (mm/dd/yyyy) }\end{array}$ & $\begin{array}{l}\text { 1,1,2-Tri- } \\
\text { chloro- } \\
\text { ethane }\end{array}$ & $\begin{array}{c}1,1,2,2-- \\
\text { Tetra- } \\
\text { chloro- } \\
\text { ethane }\end{array}$ & $\begin{array}{l}\text { 1,2-Dichloro- } \\
\text { benzene }\end{array}$ & $\begin{array}{l}\text { 1,2-Dichloro- } \\
\text { propane }\end{array}$ & $\begin{array}{l}\text { trans-1,2- } \\
\text { Dichloro- } \\
\text { ethene }\end{array}$ & $\begin{array}{c}\text { 1,2,4 -Tri- } \\
\text { chloro- } \\
\text { benzene }\end{array}$ & $\begin{array}{c}\text { 1,3-Di- } \\
\text { chloro- } \\
\text { benzene }\end{array}$ & $\begin{array}{c}\text { 1,4-Di- } \\
\text { chloro- } \\
\text { benzene }\end{array}$ & $\begin{array}{l}\text { Naph- } \\
\text { thalene }\end{array}$ \\
\hline 10/10/2001 & NA & NA & NA & NA & NA & NA & NA & NA & NA \\
\hline $10 / 22 / 2001$ & NA & NA & NA & NA & NA & NA & NA & NA & NA \\
\hline $11 / 15 / 2001$ & - & - & E0.01 & - & - & - & - & E0.01 & E0.01 \\
\hline $11 / 27 / 2001$ & - & - & E0.01 & - & - & - & - & E0.01 & E0.02 \\
\hline $12 / 09 / 2001$ & - & - & - & - & - & - & - & E0.02 & E0.02 \\
\hline $12 / 21 / 2001$ & - & - & - & - & - & - & - & E0.01 & E0.01 \\
\hline $01 / 03 / 2002$ & - & - & - & - & - & - & - & - & E0.03 \\
\hline 01/14/2002 & - & - & - & - & - & - & - & 0.07 & 0.10 \\
\hline 01/28/2002 & - & - & - & - & - & - & - & E0.01 & E0.02 \\
\hline $02 / 07 / 2002$ & - & - & - & - & - & - & - & 0.07 & 0.20 \\
\hline $02 / 19 / 2002$ & - & - & - & - & - & - & - & E0.02 & E0.02 \\
\hline $03 / 03 / 2002$ & - & - & - & - & - & - & - & E0.01 & E0.01 \\
\hline $03 / 27 / 2002$ & - & - & - & - & - & - & - & E0.02 & E0.01 \\
\hline $04 / 20 / 2002$ & - & - & - & - & - & - & - & E0.01 & E0.02 \\
\hline $05 / 14 / 2002$ & - & - & - & - & - & - & - & E0.01 & E0.01 \\
\hline $05 / 26 / 2002$ & - & - & - & - & - & - & - & E0.01 & E0.01 \\
\hline 06/07/2002 & - & - & - & - & - & - & - & - & E0.03 \\
\hline 06/19/2002 & - & - & - & - & - & - & - & E0.02 & E0.02 \\
\hline $07 / 01 / 2002$ & - & - & - & - & - & - & - & 0.07 & 0.10 \\
\hline $07 / 13 / 2002$ & - & - & - & - & - & - & - & E0.05 & 0.07 \\
\hline $07 / 25 / 2002$ & - & - & - & - & - & - & - & E0.04 & E0.04 \\
\hline 08/06/2002 & - & - & - & - & - & - & - & E0.03 & E0.05 \\
\hline $08 / 18 / 2002$ & - & - & - & - & - & - & - & E0.01 & E0.02 \\
\hline 08/30/2002 & - & - & - & - & - & - & - & 0.07 & 0.07 \\
\hline $09 / 11 / 2002$ & NA & NA & NA & NA & NA & NA & NA & NA & NA \\
\hline 09/23/2002 & NA & NA & NA & NA & NA & NA & NA & NA & NA \\
\hline $10 / 05 / 2002$ & - & - & - & - & - & - & - & E0.05 & E0.04 \\
\hline $10 / 17 / 2002$ & - & - & - & - & - & - & - & - & E0.02 \\
\hline $10 / 29 / 2002$ & - & - & - & - & - & - & - & E0.02 & E0.06 \\
\hline $11 / 10 / 2002$ & - & - & - & - & - & - & - & 0.08 & 0.10 \\
\hline $11 / 22 / 2002$ & - & - & - & - & - & - & - & E0.05 & E0.05 \\
\hline $12 / 04 / 2002$ & - & - & - & - & - & - & - & E0.02 & E0.01 \\
\hline $01 / 20 / 2003$ & - & - & - & - & - & - & - & E0.03 & E0.04 \\
\hline $02 / 04 / 2003$ & - & - & - & - & - & - & - & - & E0.01 \\
\hline $02 / 14 / 2003$ & - & - & - & - & - & - & - & E0.01 & E0.01 \\
\hline $02 / 26 / 2003$ & - & - & - & - & - & - & - & E0.03 & E0.04 \\
\hline 03/10/2003 & - & - & - & - & - & - & - & E0.02 & E0.02 \\
\hline $03 / 22 / 2003$ & - & - & - & E0.07 & - & - & - & E0.02 & E0.02 \\
\hline $04 / 03 / 2003$ & - & - & - & - & - & - & - & E0.01 & E0.01 \\
\hline
\end{tabular}


Table 9B. Analytical results for volatile organic compounds in air with high breakthrough volumes from the Sweetwater Reservoir atmospheric site, San Diego County, California.-Continued

[The site identification number is 324141117001601 . Concentrations are given in parts per billion by volume (ppbv). E, estimated value; NA, not analyzed; _, compound not detected at a concentration above the laboratory reporting level]

\begin{tabular}{|c|c|c|c|c|c|c|c|c|c|}
\hline $\begin{array}{c}\text { Date } \\
\text { (mm/dd/yyyy) }\end{array}$ & $\begin{array}{l}\text { 1,1,2-Tri- } \\
\text { chloro- } \\
\text { ethane }\end{array}$ & $\begin{array}{l}1,1,2,2-- \\
\text { Tetra- } \\
\text { chloro- } \\
\text { ethane }\end{array}$ & $\begin{array}{l}\text { 1,2-Dichloro- } \\
\text { benzene }\end{array}$ & $\begin{array}{l}\text { 1,2-Dichloro- } \\
\text { propane }\end{array}$ & $\begin{array}{l}\text { trans-1,2- } \\
\text { Dichloro- } \\
\text { ethene }\end{array}$ & $\begin{array}{c}\text { 1,2,4 -Tri- } \\
\text { chloro- } \\
\text { benzene }\end{array}$ & $\begin{array}{c}\text { 1,3-Di- } \\
\text { chloro- } \\
\text { benzene }\end{array}$ & $\begin{array}{c}\text { 1,4-Di- } \\
\text { chloro- } \\
\text { benzene }\end{array}$ & $\begin{array}{l}\text { Naph- } \\
\text { thalene }\end{array}$ \\
\hline $04 / 15 / 2003$ & - & - & - & - & - & - & - & E0.03 & E0.02 \\
\hline 05/09/2003 & - & - & - & - & - & - & - & E0.01 & E0.01 \\
\hline $05 / 21 / 2003$ & - & - & - & - & - & - & - & 0.09 & E0.05 \\
\hline $06 / 02 / 2003$ & - & - & - & - & - & - & - & E0.02 & E0.02 \\
\hline $06 / 14 / 2003$ & - & - & - & 0.09 & - & - & - & E0.01 & E0.01 \\
\hline $06 / 26 / 2003$ & - & - & - & - & - & - & - & E0.05 & E0.02 \\
\hline 07/09/2003 & NA & NA & NA & NA & NA & NA & NA & NA & NA \\
\hline 07/20/2003 & - & - & - & - & - & - & - & E0.02 & E0.02 \\
\hline 08/01/2003 & - & - & - & - & - & - & - & E0.04 & E0.05 \\
\hline 08/13/2003 & - & - & - & - & - & - & - & E0.06 & E0.05 \\
\hline $08 / 25 / 2003$ & - & - & - & - & - & - & - & E0.06 & 0.07 \\
\hline 09/06/2003 & - & - & - & - & - & - & - & 0.07 & 0.07 \\
\hline 09/18/2003 & - & - & - & - & - & - & - & E0.05 & E0.04 \\
\hline
\end{tabular}


Table 9B. Analytical results for volatile organic compounds in air with high breakthrough volumes from the Sweetwater Reservoir aatmospheric site, San Diego County, California.-Continued

[The site identification number is 324141117001601 . Concentrations are given in parts per billion by volume (ppbv). E, estimated value; NA, not analyzed; _, compound not detected at a concentration above the laboratory reporting level]

\begin{tabular}{|c|c|c|c|c|c|c|c|c|c|}
\hline $\begin{array}{c}\text { Date } \\
(\mathrm{mm} / \mathrm{dd} / \mathrm{yyyy})\end{array}$ & $\begin{array}{l}\text { trans-1,3- } \\
\text { Dichloro- } \\
\text { propene }\end{array}$ & $\begin{array}{c}\text { cis-1,3- } \\
\text { Dichloro- } \\
\text { propene }\end{array}$ & $\begin{array}{l}\text { Trichloro- } \\
\text { ethene } \\
\text { (TCE) }\end{array}$ & $\begin{array}{c}\text { Hexa- } \\
\text { chloro- } \\
\text { butadiene }\end{array}$ & $\begin{array}{l}\text { Methyl } \\
\text { acrylate }\end{array}$ & $\begin{array}{c}\text { 1,2,3,4-Tetra- } \\
\text { methyl- } \\
\text { benzene }\end{array}$ & $\begin{array}{l}\text { Ethyl tert- } \\
\text { butyl ether } \\
\text { (ETBE) }\end{array}$ & $\begin{array}{l}\text { tert-Amyl } \\
\text { methyl ether } \\
\text { (TAME) }\end{array}$ & $\begin{array}{c}\text { trans-1,4- } \\
\text { Dichloro-2- } \\
\text { butene }\end{array}$ \\
\hline $10 / 10 / 2001$ & NA & NA & NA & NA & NA & NA & NA & NA & NA \\
\hline $10 / 22 / 2001$ & NA & NA & NA & NA & NA & NA & NA & NA & NA \\
\hline $11 / 15 / 2001$ & - & - & E0.02 & - & E0.01 & E0.01 & - & - & - \\
\hline $11 / 27 / 2001$ & - & - & E0.01 & - & E0.05 & E0.01 & - & - & - \\
\hline $12 / 09 / 2001$ & - & - & - & - & E0.05 & E0.01 & - & - & - \\
\hline $12 / 21 / 2001$ & - & - & - & - & - & E0.01 & - & - & - \\
\hline $01 / 03 / 2002$ & - & - & E0.01 & - & - & - & E0.05 & - & - \\
\hline $01 / 14 / 2002$ & - & - & 0.07 & - & - & E0.01 & - & E0.04 & - \\
\hline $01 / 28 / 2002$ & - & - & E0.01 & - & - & E0.01 & - & - & - \\
\hline $02 / 07 / 2002$ & - & - & E0.01 & - & - & E0.01 & - & - & - \\
\hline $02 / 19 / 2002$ & - & - & E0.01 & - & - & E0.01 & - & - & - \\
\hline 03/03/2002 & - & - & E0.01 & - & - & E0.01 & - & - & - \\
\hline $03 / 27 / 2002$ & - & - & E0.01 & - & - & E0.01 & - & - & - \\
\hline $04 / 20 / 2002$ & - & - & E0.01 & - & - & E0.01 & E0.01 & - & - \\
\hline $05 / 14 / 2002$ & - & - & E0.01 & - & - & E0.01 & - & - & - \\
\hline 05/26/2002 & - & - & - & - & - & - & - & - & - \\
\hline 06/07/2002 & - & - & - & - & - & E0.01 & - & - & - \\
\hline 06/19/2002 & - & - & E0.03 & - & - & E0.01 & - & - & - \\
\hline 07/01/2002 & - & - & - & - & - & E0.01 & - & - & - \\
\hline $07 / 13 / 2002$ & - & - & E0.01 & - & - & E0.01 & E0.01 & - & - \\
\hline $07 / 25 / 2002$ & - & - & E0.02 & - & E0.04 & E0.01 & E0.01 & - & - \\
\hline 08/06/2002 & - & - & E0.03 & - & - & E0.01 & E0.01 & - & - \\
\hline $08 / 18 / 2002$ & - & - & - & - & - & - & - & - & - \\
\hline $08 / 30 / 2002$ & - & - & E0.02 & - & E0.02 & E0.01 & - & E0.03 & - \\
\hline 09/11/2002 & NA & NA & NA & NA & NA & NA & NA & NA & NA \\
\hline $09 / 23 / 2002$ & NA & NA & NA & NA & NA & NA & NA & NA & NA \\
\hline $10 / 05 / 2002$ & - & - & E0.02 & - & E0.02 & E0.01 & E0.01 & - & - \\
\hline $10 / 17 / 2002$ & - & - & - & - & - & - & E0.04 & - & - \\
\hline $10 / 29 / 2002$ & - & - & E0.07 & - & - & E0.01 & E0.03 & - & - \\
\hline $11 / 10 / 2002$ & - & - & - & - & - & E0.02 & E0.01 & - & - \\
\hline $11 / 22 / 2002$ & - & - & 0.06 & - & - & E0.01 & E0.01 & - & - \\
\hline $12 / 04 / 2002$ & - & - & E0.01 & - & - & E0.01 & - & - & - \\
\hline $01 / 20 / 2003$ & - & - & E0.02 & - & - & E0.01 & - & - & - \\
\hline $02 / 04 / 2003$ & - & - & E0.01 & - & - & E0.01 & E0.03 & - & - \\
\hline $02 / 14 / 2003$ & - & - & E0.01 & - & - & E0.01 & - & - & - \\
\hline $02 / 26 / 2003$ & - & - & 0.25 & - & - & E0.01 & - & - & - \\
\hline 03/10/2003 & - & - & - & - & - & E0.01 & - & - & - \\
\hline $03 / 22 / 2003$ & - & - & - & - & - & E0.01 & - & - & - \\
\hline $04 / 03 / 2003$ & - & - & E0.01 & - & - & E0.01 & - & - & - \\
\hline $04 / 15 / 2003$ & - & - & E0.01 & - & - & E0.01 & - & - & - \\
\hline
\end{tabular}


Table 9B. Analytical results for volatile organic compounds in air with high breakthrough volumes from the Sweetwater Reservoir aatmospheric site, San Diego County, California.-Continued

[The site identification number is 324141117001601 . Concentrations are given in parts per billion by volume (ppbv). E, estimated value; NA, not analyzed; _, compound not detected at a concentration above the laboratory reporting level]

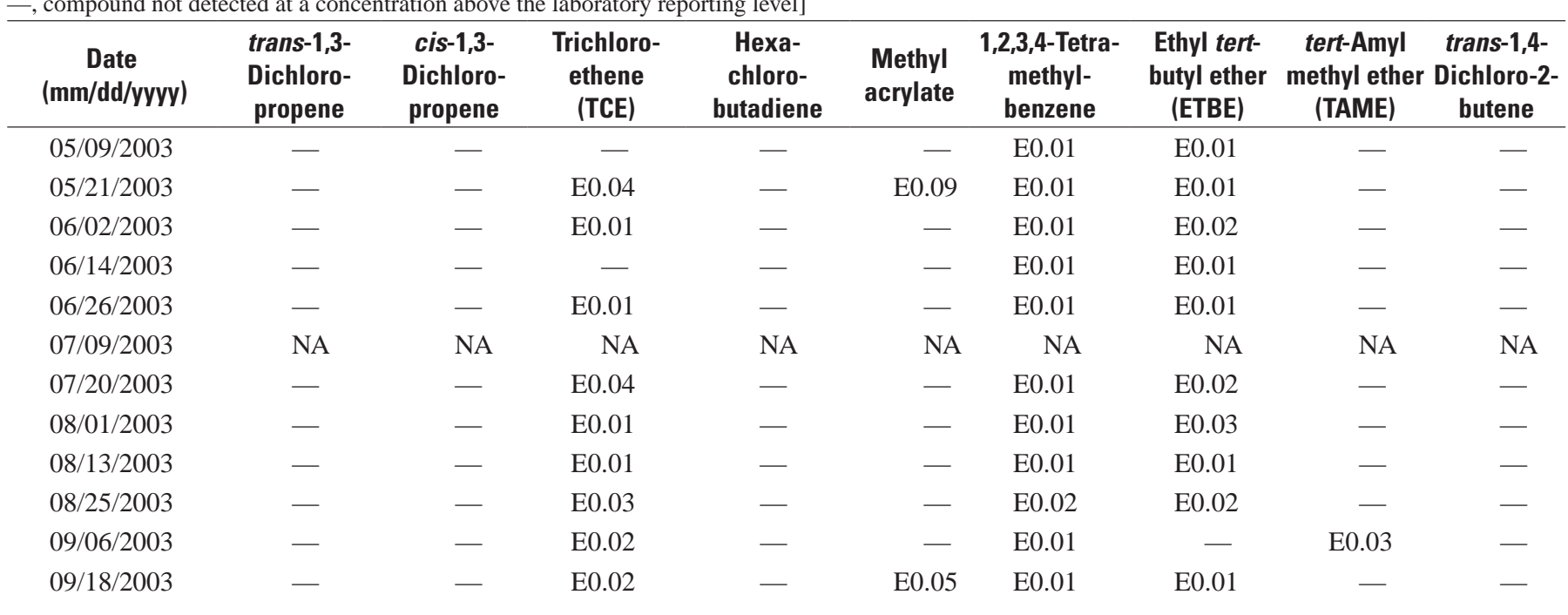


Table 9B. Analytical results for volatile organic compounds in air with high breakthrough volumes from the Sweetwater Reservoir atmospheric site, San Diego County, California.-Continued

[The site identification number is 324141117001601 . Concentrations are given in parts per billion by volume (ppbv). E, estimated value; NA, not analyzed; _, compound not detected at a concentration above the laboratory reporting level]

\begin{tabular}{|c|c|c|c|c|c|c|c|c|c|}
\hline $\begin{array}{c}\text { Date } \\
\text { (mm/dd/yyyy) }\end{array}$ & $\begin{array}{l}\text { Ethylmeth- } \\
\text { acrylate }\end{array}$ & $\begin{array}{l}\text { Carbon } \\
\text { disulfide }\end{array}$ & $\begin{array}{c}\text { cis-1,2- } \\
\text { Dichloro- } \\
\text { ethene }\end{array}$ & $\begin{array}{c}\text { 2-Hexanone } \\
\text { (MBK) }\end{array}$ & $\begin{array}{l}\text { Ethenyl- } \\
\text { benzene } \\
\text { (Styrene) }\end{array}$ & o-Xylene & $\begin{array}{c}\text { 1,1-Di- } \\
\text { chloro- } \\
\text { propene }\end{array}$ & $\begin{array}{l}\text { 2,2-Di- } \\
\text { chloro- } \\
\text { propene }\end{array}$ & $\begin{array}{c}\text { 1,3-Di- } \\
\text { chloro- } \\
\text { propene }\end{array}$ \\
\hline $10 / 10 / 2001$ & NA & NA & NA & NA & NA & NA & NA & NA & NA \\
\hline $10 / 22 / 2001$ & NA & NA & NA & NA & NA & NA & NA & NA & NA \\
\hline $11 / 15 / 2001$ & - & E0.02 & - & - & - & 0.11 & - & - & - \\
\hline $11 / 27 / 2001$ & - & - & - & - & - & 0.15 & - & - & - \\
\hline $12 / 21 / 2001$ & - & - & - & E0.01 & E0.02 & E0.03 & - & - & - \\
\hline 01/03/2002 & - & E0.03 & - & E0.03 & E0.04 & E0.03 & - & - & - \\
\hline $01 / 14 / 2002$ & - & E0.02 & - & E0.03 & E0.08 & 0.47 & - & - & - \\
\hline $01 / 14 / 2002$ & - & E0.02 & - & E0.03 & 0.09 & 0.42 & - & - & - \\
\hline $01 / 28 / 2002$ & - & - & - & E0.01 & E0.03 & 0.10 & - & - & - \\
\hline $03 / 27 / 2002$ & - & - & - & E0.01 & - & E0.07 & 0.14 & - & - \\
\hline $04 / 20 / 2002$ & - & - & - & E0.01 & - & E0.07 & - & - & - \\
\hline $05 / 14 / 2002$ & - & E0.05 & - & E0.01 & - & E0.07 & - & - & - \\
\hline $05 / 26 / 2002$ & - & E0.06 & - & E0.01 & - & E0.05 & - & - & - \\
\hline $06 / 07 / 2002$ & - & E0.03 & - & 0.20 & E0.02 & 0.16 & - & - & - \\
\hline $06 / 19 / 2002$ & - & - & - & E0.01 & E0.04 & 0.15 & - & - & - \\
\hline 07/01/2002 & - & - & - & E0.07 & E0.06 & 0.26 & - & - & - \\
\hline $07 / 13 / 2002$ & - & - & - & E0.02 & E0.03 & 0.19 & - & - & - \\
\hline $07 / 25 / 2002$ & - & - & - & E0.02 & E0.02 & 0.12 & - & - & - \\
\hline 08/06/2002 & - & - & - & E0.02 & E0.04 & 0.15 & - & - & - \\
\hline $10 / 29 / 2002$ & - & - & - & E0.03 & E0.05 & 0.23 & - & - & - \\
\hline $11 / 10 / 2002$ & - & - & - & E0.03 & 0.11 & 0.63 & - & - & - \\
\hline $11 / 22 / 2002$ & - & - & - & E0.02 & E0.07 & 0.32 & - & - & - \\
\hline $12 / 04 / 2002$ & - & - & - & E0.02 & E0.02 & 0.09 & - & - & - \\
\hline $01 / 20 / 2003$ & - & - & - & E0.01 & E0.03 & 0.18 & - & - & - \\
\hline $02 / 04 / 2003$ & - & - & - & E0.01 & E0.01 & E0.01 & - & - & - \\
\hline $02 / 14 / 2003$ & - & - & - & E0.01 & E0.01 & E0.07 & - & - & - \\
\hline $02 / 26 / 2003$ & - & E0.03 & - & 0.13 & 0.11 & 0.24 & - & - & - \\
\hline 03/10/2003 & - & - & - & E0.06 & E0.02 & 0.13 & - & - & - \\
\hline $03 / 22 / 2003$ & - & - & - & E0.07 & E0.02 & 0.19 & - & - & - \\
\hline $04 / 03 / 2003$ & - & - & - & E0.02 & E0.02 & 0.10 & - & - & - \\
\hline
\end{tabular}


Table 9B. Analytical results for volatile organic compounds in air with high breakthrough volumes from the Sweetwater Reservoir atmospheric site, San Diego County, California.-Continued

[The site identification number is 324141117001601 . Concentrations are given in parts per billion by volume (ppbv). E, estimated value; NA, not analyzed; _, compound not detected at a concentration above the laboratory reporting level]

\begin{tabular}{|c|c|c|c|c|c|c|c|c|c|}
\hline $\begin{array}{c}\text { Date } \\
\text { (mm/dd/yyyy) }\end{array}$ & $\begin{array}{l}\text { Ethylmeth- } \\
\text { acrylate }\end{array}$ & $\begin{array}{l}\text { Carbon } \\
\text { disulfide }\end{array}$ & $\begin{array}{c}\text { cis-1,2- } \\
\text { Dichloro- } \\
\text { ethene }\end{array}$ & $\begin{array}{c}\text { 2-Hexanone } \\
\text { (MBK) }\end{array}$ & $\begin{array}{l}\text { Ethenyl- } \\
\text { benzene } \\
\text { (Styrene) }\end{array}$ & o-Xylene & $\begin{array}{l}\text { 1,1-Di- } \\
\text { chloro- } \\
\text { propene }\end{array}$ & $\begin{array}{c}\text { 2,2-Di- } \\
\text { chloro- } \\
\text { propene }\end{array}$ & $\begin{array}{c}\text { 1,3-Di- } \\
\text { chloro- } \\
\text { propene }\end{array}$ \\
\hline $04 / 15 / 2003$ & - & E0.03 & - & E0.03 & E0.01 & 0.10 & - & - & - \\
\hline $05 / 21 / 2003$ & - & - & - & E0.03 & E0.05 & 0.36 & - & - & - \\
\hline 06/02/2003 & - & - & - & E0.02 & E0.02 & 0.13 & - & - & - \\
\hline $06 / 26 / 2003$ & - & E0.02 & - & E0.06 & E0.03 & 0.19 & - & - & - \\
\hline 07/09/2003 & NA & NA & NA & NA & NA & NA & NA & NA & NA \\
\hline 07/20/2003 & - & - & - & E0.03 & E0.02 & 0.11 & - & - & - \\
\hline 08/01/2003 & - & E0.03 & - & E0.01 & E0.02 & 0.13 & - & - & - \\
\hline $08 / 13 / 2003$ & - & E0.02 & - & E0.02 & E0.03 & 0.20 & - & - & - \\
\hline
\end{tabular}


Table 9B. Analytical results for volatile organic compounds in air with high breakthrough volumes from the Sweetwater Reservoir atmospheric site, San Diego County, California.-Continued

[The site identification number is 324141117001601 . Concentrations are given in parts per billion by volume (ppbv). E, estimated value; NA, not analyzed; _, compound not detected at a concentration above the laboratory reporting level]

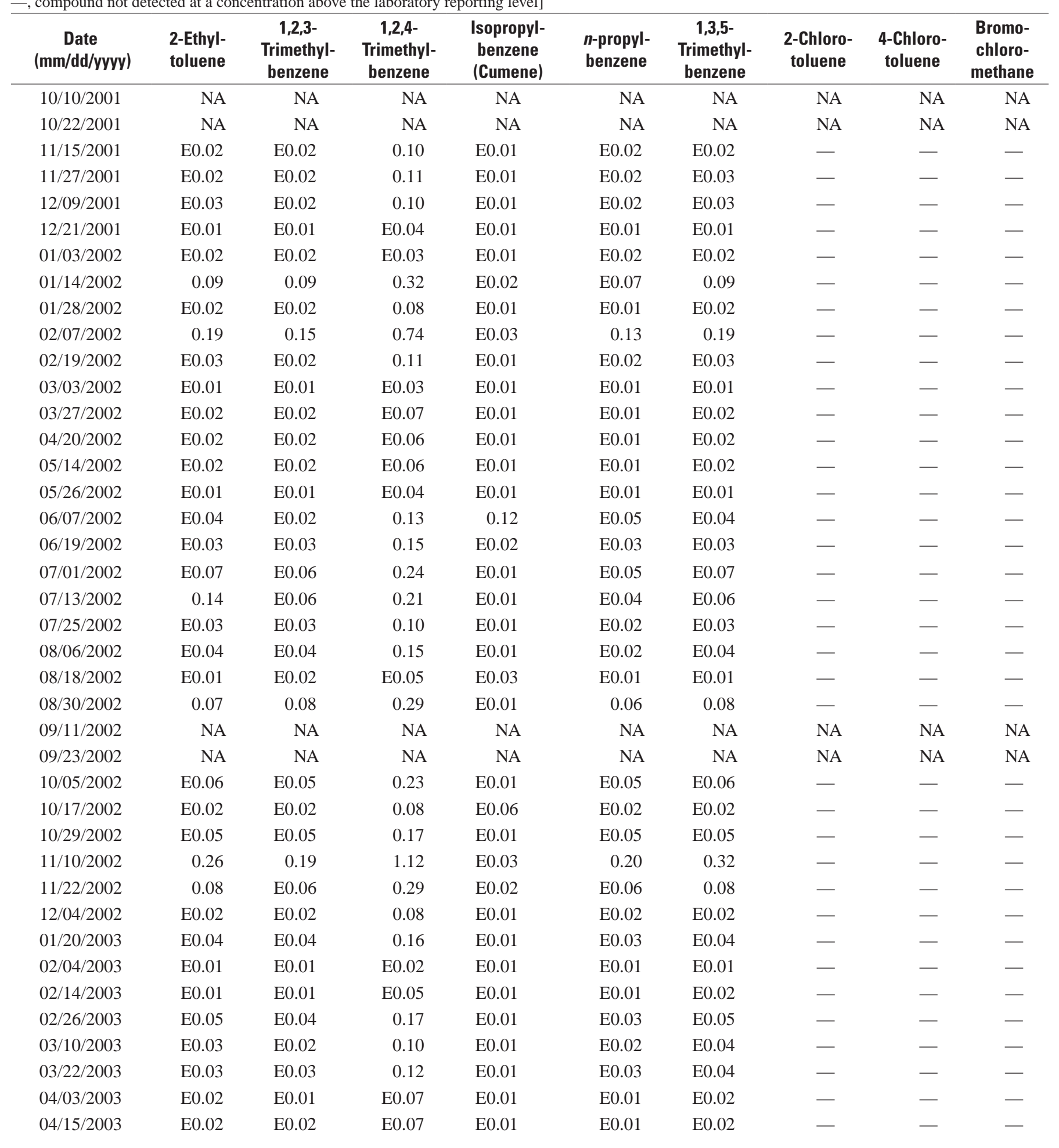


Table 9B. Analytical results for volatile organic compounds in air with high breakthrough volumes from the Sweetwater Reservoir atmospheric site, San Diego County, California.-Continued

[The site identification number is 324141117001601 . Concentrations are given in parts per billion by volume (ppbv). E, estimated value; NA, not analyzed; —, compound not detected at a concentration above the laboratory reporting level]

\begin{tabular}{|c|c|c|c|c|c|c|c|c|c|}
\hline $\begin{array}{c}\text { Date } \\
\text { (mm/dd/yyyy) }\end{array}$ & $\begin{array}{l}\text { 2-Ethyl- } \\
\text { toluene }\end{array}$ & $\begin{array}{c}\text { 1,2,3- } \\
\text { Trimethyl- } \\
\text { benzene }\end{array}$ & $\begin{array}{c}\text { 1,2,4- } \\
\text { Trimethyl- } \\
\text { benzene }\end{array}$ & $\begin{array}{c}\text { Isopropyl- } \\
\text { benzene } \\
\text { (Cumene) }\end{array}$ & $\begin{array}{l}n \text {-propyl- } \\
\text { benzene }\end{array}$ & $\begin{array}{c}\text { 1,3,5- } \\
\text { Trimethyl- } \\
\text { benzene }\end{array}$ & $\begin{array}{l}\text { 2-Chloro- } \\
\text { toluene }\end{array}$ & $\begin{array}{l}\text { 4-Chloro- } \\
\text { toluene }\end{array}$ & $\begin{array}{l}\text { Bromo- } \\
\text { chloro- } \\
\text { methane }\end{array}$ \\
\hline 05/09/2003 & E0.01 & E0.01 & E0.04 & E0.01 & E0.01 & E0.01 & - & - & - \\
\hline 06/02/2003 & E0.03 & E0.02 & 0.09 & E0.01 & E0.02 & E0.03 & - & - & - \\
\hline $06 / 14 / 2003$ & E0.01 & E0.01 & 0.05 & E0.01 & E0.01 & E0.02 & - & - & - \\
\hline $07 / 09 / 2003$ & NA & NA & NA & NA & NA & NA & NA & NA & NA \\
\hline $07 / 20 / 2003$ & E0.03 & E0.03 & 0.09 & E0.01 & E0.02 & E0.02 & - & - & 一 \\
\hline 08/01/2003 & E0.03 & E0.04 & 0.10 & E0.01 & E0.02 & E0.03 & - & - & - \\
\hline $08 / 13 / 2003$ & E0.05 & E0.05 & 0.17 & E0.01 & E0.03 & E0.05 & - & - & - \\
\hline $08 / 25 / 2003$ & E0.04 & E0.05 & 0.12 & E0.01 & E0.03 & E0.04 & - & - & - \\
\hline
\end{tabular}


Table 9B. Analytical results for volatile organic compounds in air with high breakthrough volumes from the Sweetwater Reservoir atmospheric site, San Diego County, California.-Continued

[The site identification number is 324141117001601 . Concentrations are given in parts per billion by volume (ppbv). E, estimated value; NA, not analyzed; _, compound not detected at a concentration above the laboratory reporting level]

\begin{tabular}{|c|c|c|c|c|c|c|c|c|c|}
\hline $\begin{array}{c}\text { Date } \\
\text { (mm/dd/yyyy) }\end{array}$ & $\begin{array}{l}n \text {-Butyl- } \\
\text { benzene }\end{array}$ & $\begin{array}{c}\text { sec-Butyl- } \\
\text { benzene }\end{array}$ & $\begin{array}{l}\text { tert-Butyl- } \\
\text { benzene }\end{array}$ & $\begin{array}{l}\text { 1-Isopropyl- } \\
\text { 4-methyl- } \\
\text { benzene }\end{array}$ & $\begin{array}{c}\text { 1,2,3-- } \\
\text { Trichloro- } \\
\text { propene }\end{array}$ & $\begin{array}{c}1,1,1,2- \\
\text { Tetrachloro- } \\
\text { ethane }\end{array}$ & $\begin{array}{c}1,2,3- \\
\text { Trichloro- } \\
\text { benzene }\end{array}$ & $\begin{array}{c}1,2- \\
\text { Dibromo- } \\
\text { ethane }\end{array}$ & $\begin{array}{l}\text { Methyl tert- } \\
\text { butyl ether } \\
\text { (MTBE) }\end{array}$ \\
\hline $10 / 10 / 2001$ & NA & NA & NA & NA & NA & NA & NA & NA & NA \\
\hline $10 / 22 / 2001$ & NA & NA & NA & NA & NA & NA & NA & NA & NA \\
\hline $11 / 15 / 2001$ & E0.01 & E0.01 & - & E0.03 & - & - & - & - & 0.88 \\
\hline $11 / 27 / 2001$ & E0.01 & E0.01 & - & E0.01 & - & - & - & - & 0.98 \\
\hline $12 / 09 / 2001$ & E0.01 & E0.01 & - & E0.02 & - & - & - & - & 0.82 \\
\hline $12 / 21 / 2001$ & E0.01 & - & - & E0.01 & - & - & - & - & E0.11 \\
\hline $01 / 03 / 2002$ & - & - & - & E0.01 & - & - & - & - & E0.05 \\
\hline $01 / 14 / 2002$ & - & E0.01 & - & E0.04 & - & - & - & - & 3.43 \\
\hline $01 / 28 / 2002$ & E0.01 & - & - & E0.01 & - & - & - & - & 0.21 \\
\hline $02 / 07 / 2002$ & - & - & - & E0.04 & - & - & - & - & 2.62 \\
\hline $02 / 19 / 2002$ & E0.01 & E0.01 & - & E0.01 & - & - & - & - & 0.78 \\
\hline $03 / 03 / 2002$ & E0.01 & - & - & E0.01 & - & - & - & - & 0.26 \\
\hline 03/27/2002 & E0.01 & E0.01 & - & E0.01 & - & - & - & - & 0.54 \\
\hline $04 / 20 / 2002$ & E0.01 & E0.01 & - & E0.01 & - & - & - & - & 0.36 \\
\hline $05 / 14 / 2002$ & E0.01 & - & - & E0.01 & - & - & - & - & 0.34 \\
\hline $05 / 26 / 2002$ & E0.01 & - & - & E0.01 & - & - & - & - & 0.25 \\
\hline 06/07/2002 & E0.01 & 0.06 & - & E0.01 & - & - & - & - & 0.74 \\
\hline $06 / 19 / 2002$ & E0.01 & E0.01 & - & E0.01 & - & - & - & - & 0.35 \\
\hline $07 / 01 / 2002$ & E0.03 & E0.01 & - & E0.05 & - & - & - & - & 2.00 \\
\hline 07/13/2002 & E0.01 & E0.01 & - & E0.05 & - & - & - & - & 0.80 \\
\hline $07 / 25 / 2002$ & E0.01 & E0.01 & - & E0.03 & - & - & - & - & 0.87 \\
\hline 08/06/2002 & E0.01 & - & - & E0.03 & - & - & - & - & 0.76 \\
\hline $08 / 18 / 2002$ & E0.01 & - & - & E0.01 & - & - & - & - & 0.29 \\
\hline 08/30/2002 & E0.02 & E0.01 & - & E0.05 & - & - & - & - & 1.51 \\
\hline $09 / 11 / 2002$ & NA & NA & NA & NA & NA & NA & NA & NA & NA \\
\hline 09/23/2002 & NA & NA & NA & NA & NA & NA & NA & NA & NA \\
\hline $10 / 05 / 2002$ & E0.01 & - & - & E0.03 & - & - & - & - & 1.86 \\
\hline 10/17/2002 & E0.01 & - & - & E0.04 & - & - & - & - & 0.56 \\
\hline $10 / 29 / 2002$ & E0.02 & - & - & E0.04 & - & E0.02 & - & - & 0.68 \\
\hline $11 / 10 / 2002$ & E0.04 & E0.02 & - & 0.81 & - & - & - & - & 1.92 \\
\hline $11 / 22 / 2002$ & E0.02 & E0.01 & - & E0.02 & - & - & - & - & 2.17 \\
\hline $12 / 04 / 2002$ & E0.01 & - & - & E0.01 & - & - & - & - & 0.54 \\
\hline $01 / 20 / 2003$ & E0.01 & - & - & E0.02 & - & - & - & - & 0.46 \\
\hline $02 / 04 / 2003$ & E0.01 & - & - & E0.01 & - & - & - & - & E0.01 \\
\hline $02 / 14 / 2003$ & E0.01 & E0.01 & - & E0.02 & - & - & - & - & 0.19 \\
\hline $02 / 26 / 2003$ & E0.01 & E0.01 & - & E0.02 & - & - & - & - & 0.46 \\
\hline $03 / 10 / 2003$ & E0.01 & - & - & E0.01 & - & - & - & - & 0.54 \\
\hline $03 / 22 / 2003$ & E0.01 & - & - & E0.01 & - & - & - & - & 0.68 \\
\hline $04 / 03 / 2003$ & E0.01 & - & - & E0.01 & - & - & - & - & 0.30 \\
\hline $04 / 15 / 2003$ & E0.01 & - & - & E0.01 & - & - & - & - & 0.39 \\
\hline
\end{tabular}


Table 9B. Analytical results for volatile organic compounds in air with high breakthrough volumes from the Sweetwater Reservoir atmospheric site, San Diego County, California.-Continued

[The site identification number is 324141117001601 . Concentrations are given in parts per billion by volume (ppbv). E, estimated value; NA, not analyzed; _, compound not detected at a concentration above the laboratory reporting level]

\begin{tabular}{|c|c|c|c|c|c|c|c|c|c|}
\hline $\begin{array}{c}\text { Date } \\
\text { (mm/dd/yyyy) }\end{array}$ & $\begin{array}{l}n \text {-Butyl- } \\
\text { benzene }\end{array}$ & $\begin{array}{l}\text { sec-Butyl- } \\
\text { benzene }\end{array}$ & $\begin{array}{l}\text { tert-Butyl- } \\
\text { benzene }\end{array}$ & $\begin{array}{c}\text { 1-Isopropyl- } \\
\text { 4-methyl- } \\
\text { benzene }\end{array}$ & $\begin{array}{c}\text { 1,2,3-- } \\
\text { Trichloro- } \\
\text { propene }\end{array}$ & $\begin{array}{c}\text { 1,1,1,2- } \\
\text { Tetrachloro- } \\
\text { ethane }\end{array}$ & $\begin{array}{c}\text { 1,2,3- } \\
\text { Trichloro- } \\
\text { benzene }\end{array}$ & $\begin{array}{c}\text { 1,2- } \\
\text { Dibromo- } \\
\text { ethane }\end{array}$ & $\begin{array}{c}\text { Methyl tert- } \\
\text { butyl ether } \\
\text { (MTBE) }\end{array}$ \\
\hline $05 / 09 / 2003$ & E0.01 & - & - & E0.01 & - & - & - & - & 0.19 \\
\hline $05 / 21 / 2003$ & E0.01 & E0.01 & 0.05 & E0.03 & - & - & - & - & 1.40 \\
\hline 06/02/2003 & E0.01 & E0.01 & - & E0.01 & - & - & - & - & 0.33 \\
\hline $06 / 14 / 2003$ & E0.01 & - & - & E0.01 & - & - & - & - & 0.16 \\
\hline $06 / 26 / 2003$ & E0.01 & E0.01 & - & E0.04 & - & - & - & - & 0.52 \\
\hline 07/09/2003 & NA & NA & NA & NA & NA & NA & NA & NA & NA \\
\hline $07 / 20 / 2003$ & E0.01 & E0.01 & - & E0.03 & - & - & - & - & 0.47 \\
\hline 08/01/2003 & E0.01 & E0.01 & - & E0.03 & - & - & - & - & 0.40 \\
\hline $08 / 13 / 2003$ & E0.01 & E0.01 & - & 0.07 & - & E0.03 & - & - & 0.51 \\
\hline $08 / 25 / 2003$ & E0.01 & - & - & 0.07 & - & - & - & - & 0.55 \\
\hline $09 / 6 / 2003$ & E0.02 & E0.01 & - & E0.05 & - & - & - & - & 1.51 \\
\hline $09 / 18 / 2003$ & E0.01 & - & - & E0.03 & - & - & - & - & 1.84 \\
\hline
\end{tabular}


Table 9B. Analytical results for volatile organic compounds in air with high breakthrough volumes from the Sweetwater Reservoir atmospheric site, San Diego County, California.-Continued

[The site identification number is 324141117001601 . Concentrations are given in parts per billion by volume (ppbv). E, estimated value; NA, not analyzed; —, compound not detected at a concentration above the laboratory reporting level]

\begin{tabular}{|c|c|c|c|c|c|c|c|c|c|}
\hline $\begin{array}{c}\text { Date } \\
\text { (mm/dd/yyyy) }\end{array}$ & $\begin{array}{l}\text { 3-Chloro- } \\
\text { 1-propene }\end{array}$ & $\begin{array}{c}\text { 4-Methyl-2- } \\
\text { pentanone } \\
\text { (MIBK) }\end{array}$ & Acetone & $\begin{array}{l}\text { Bromo- } \\
\text { benzene }\end{array}$ & $\begin{array}{l}\text { Diethyl } \\
\text { ether }\end{array}$ & $\begin{array}{c}\text { Diisopropyl } \\
\text { ether } \\
\text { (DIPE) }\end{array}$ & $\begin{array}{c}\text { Methyl- } \\
\text { acrylonitrile }\end{array}$ & $\begin{array}{c}\text { 2-Butanone } \\
\text { (Methyl } \\
\text { ethyl } \\
\text { ketone) }\end{array}$ & $\begin{array}{c}\text { Methyl- } \\
\text { meth- } \\
\text { acrylate }\end{array}$ \\
\hline $10 / 10 / 2001$ & NA & NA & NA & NA & NA & NA & NA & NA & NA \\
\hline $10 / 22 / 2001$ & NA & NA & NA & NA & NA & NA & NA & NA & NA \\
\hline $11 / 15 / 2001$ & - & - & - & - & - & - & - & 0.18 & - \\
\hline $11 / 27 / 2001$ & - & - & 1.08 & - & - & - & - & 0.18 & - \\
\hline $12 / 09 / 2001$ & - & E0.01 & - & - & - & - & - & 0.23 & - \\
\hline $12 / 21 / 2001$ & - & E0.01 & 0.47 & - & - & - & - & E0.08 & - \\
\hline $01 / 03 / 2002$ & - & - & 2.30 & - & - & - & - & 0.22 & - \\
\hline $01 / 14 / 2002$ & - & E0.06 & 3.58 & - & - & - & - & 1.12 & - \\
\hline $01 / 28 / 2002$ & - & - & 1.50 & - & - & - & - & E0.09 & - \\
\hline 02/07/2002 & - & - & 1.03 & - & - & - & - & 0.81 & - \\
\hline $02 / 19 / 2002$ & - & E0.02 & 1.02 & - & - & - & - & 0.22 & - \\
\hline 03/03/2002 & - & E0.03 & - & - & - & - & - & E0.08 & - \\
\hline $03 / 27 / 2002$ & - & E0.01 & - & - & - & - & - & 0.21 & - \\
\hline $04 / 20 / 2002$ & - & E0.01 & 1.42 & - & E0.02 & - & - & 0.19 & - \\
\hline $05 / 14 / 2002$ & - & E0.02 & 0.46 & - & - & - & - & 0.31 & - \\
\hline $05 / 26 / 2002$ & - & E0.01 & 1.25 & - & - & - & - & 0.36 & - \\
\hline 06/07/2002 & - & E0.09 & 3.78 & - & - & - & - & 0.32 & - \\
\hline 06/19/2002 & - & E0.02 & 0.54 & - & - & - & - & 0.30 & - \\
\hline 07/01/2002 & - & E0.07 & 3.59 & - & - & - & - & 0.78 & - \\
\hline $07 / 13 / 2002$ & - & E0.05 & 2.57 & - & - & - & - & 0.59 & - \\
\hline $07 / 25 / 2002$ & - & E0.03 & 1.10 & - & - & - & - & 0.45 & - \\
\hline 08/06/2002 & - & E0.02 & 1.31 & - & - & - & - & 0.45 & - \\
\hline 08/18/2002 & - & - & 1.56 & - & - & E0.02 & - & 0.31 & - \\
\hline 08/30/2002 & - & E0.06 & 2.24 & - & - & - & - & 0.32 & - \\
\hline 09/11/2002 & NA & NA & NA & NA & NA & NA & NA & NA & NA \\
\hline $09 / 23 / 2002$ & NA & NA & NA & NA & NA & NA & NA & NA & NA \\
\hline $10 / 05 / 2002$ & - & E0.06 & 1.52 & - & - & - & - & 0.59 & - \\
\hline $10 / 17 / 2002$ & - & E0.01 & 2.57 & - & - & - & - & 0.46 & - \\
\hline $10 / 29 / 2002$ & - & E0.04 & 2.45 & - & - & - & - & 0.63 & - \\
\hline $11 / 10 / 2002$ & - & E0.04 & 1.61 & - & - & - & - & 0.40 & - \\
\hline $11 / 22 / 2002$ & - & E0.08 & 1.05 & - & - & - & - & 0.66 & - \\
\hline $12 / 04 / 2002$ & - & E0.01 & - & - & - & - & - & E0.12 & - \\
\hline $01 / 20 / 2003$ & - & E0.02 & 0.21 & - & - & - & - & 0.48 & - \\
\hline $02 / 04 / 2003$ & - & E0.09 & 0.60 & - & - & - & - & E0.14 & - \\
\hline $02 / 14 / 2003$ & - & E0.01 & 0.51 & - & - & - & - & E0.09 & - \\
\hline $02 / 26 / 2003$ & - & E0.04 & 1.15 & - & - & - & - & 0.48 & - \\
\hline 03/10/2003 & - & 0.10 & 2.67 & - & - & - & - & 0.34 & - \\
\hline $03 / 22 / 2003$ & - & 0.16 & 0.79 & - & - & - & - & 0.42 & - \\
\hline 04/03/2003 & - & E0.03 & 1.22 & - & - & - & - & 0.27 & - \\
\hline
\end{tabular}


Table 9B. Analytical results for volatile organic compounds in air with high breakthrough volumes from the Sweetwater Reservoir atmospheric site, San Diego County, California.-Continued

[The site identification number is 324141117001601 . Concentrations are given in parts per billion by volume (ppbv). E, estimated value; NA, not analyzed; _, compound not detected at a concentration above the laboratory reporting level]

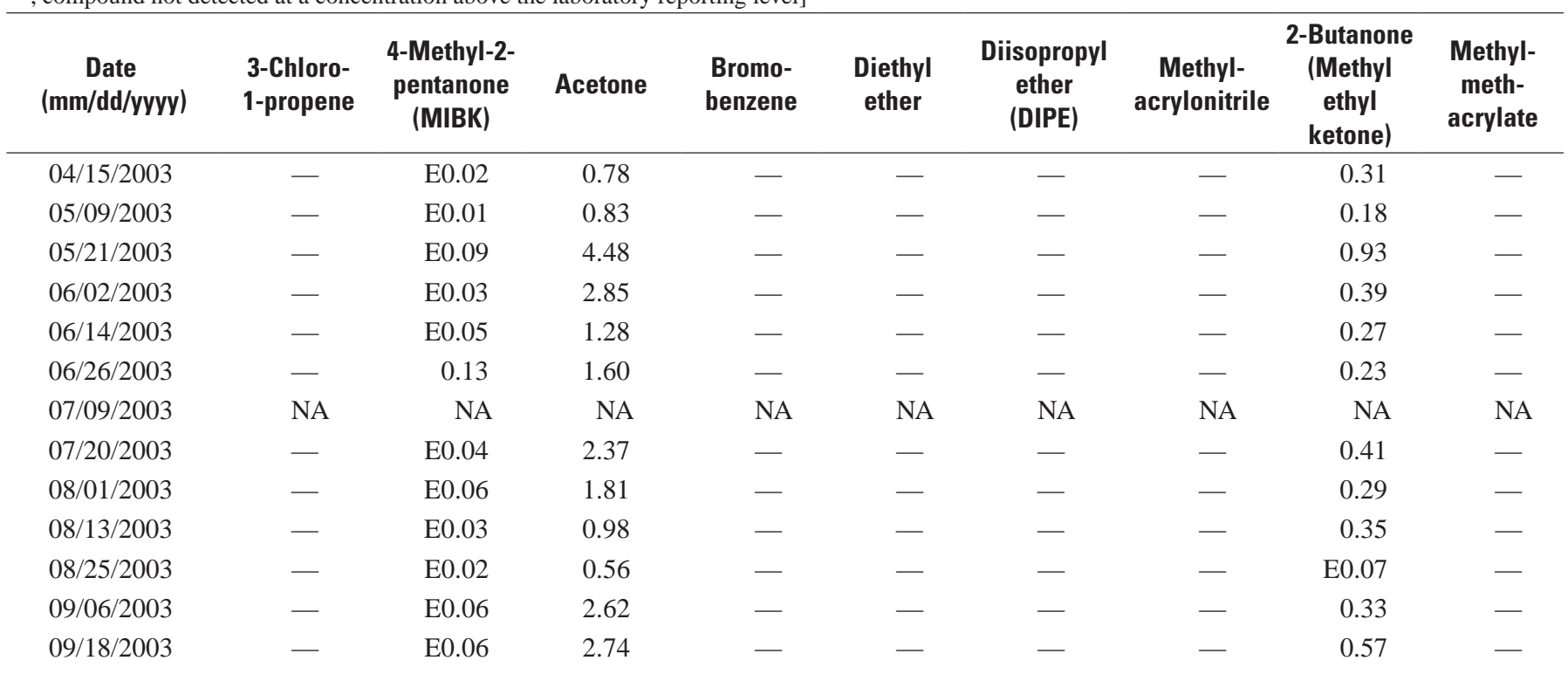


Table 9B. Analytical results for volatile organic compounds in air with high breakthrough volumes from the Sweetwater Reservoir atmospheric site, San Diego County, California.-Continued

[The site identification number is 324141117001601 . Concentrations are given in parts per billion by volume (ppbv). E, estimated value; NA, not analyzed; —, compound not detected at a concentration above the laboratory reporting level]

\begin{tabular}{|c|c|c|c|c|c|c|c|c|}
\hline $\begin{array}{c}\text { Date } \\
\text { (mm/dd/yyyy) }\end{array}$ & $\begin{array}{c}\text { Tetra- } \\
\text { hydrofuran }\end{array}$ & $\begin{array}{c}\text { 1,2,-Dibromo- } \\
\text { 3-chloro- } \\
\text { propene } \\
\text { (DBCP) }\end{array}$ & $\begin{array}{c}m \text { - and } p-- \\
\text { Xylene }\end{array}$ & $\begin{array}{c}1,2,3,5- \\
\text { Tetra- } \\
\text { methyl- } \\
\text { benzene }\end{array}$ & $\begin{array}{c}1,2,4,5- \\
\text { Tetra- } \\
\text { methyl- } \\
\text { benzene }\end{array}$ & $\begin{array}{l}\text { Methyl- } \\
\text { acetate }\end{array}$ & $\begin{array}{l}\text { tert-Amyl } \\
\text { alcohol }\end{array}$ & $\begin{array}{l}\text { tert-Butyl } \\
\text { alcohol }\end{array}$ \\
\hline $10 / 10 / 2001$ & NA & NA & NA & NA & NA & NA & NA & NA \\
\hline $10 / 22 / 2001$ & NA & NA & NA & NA & NA & NA & NA & NA \\
\hline $11 / 15 / 2001$ & - & - & 0.34 & E0.01 & E0.01 & - & - & E0.05 \\
\hline $11 / 27 / 2001$ & - & - & 0.52 & E0.01 & E0.01 & - & - & E0.06 \\
\hline $12 / 09 / 2001$ & - & - & 0.38 & E0.01 & E0.01 & - & E0.01 & E0.09 \\
\hline $12 / 21 / 2001$ & - & - & E0.08 & E0.01 & E0.01 & - & - & E0.05 \\
\hline $01 / 03 / 2002$ & - & - & E0.08 & - & - & - & E0.04 & 0.24 \\
\hline $01 / 14 / 2002$ & E0.03 & - & 1.19 & E0.04 & E0.03 & - & E0.08 & 0.29 \\
\hline $01 / 28 / 2002$ & - & - & 0.26 & E0.01 & E0.01 & - & E0.01 & - \\
\hline $02 / 07 / 2002$ & - & - & 2.33 & E0.03 & E0.03 & - & - & E0.10 \\
\hline $02 / 19 / 2002$ & - & - & 0.42 & E0.01 & E0.01 & - & E0.01 & E0.08 \\
\hline 03/03/2002 & - & - & 0.13 & E0.01 & E0.01 & - & - & - \\
\hline 03/27/2002 & - & - & 0.20 & E0.01 & E0.01 & - & E0.01 & E0.08 \\
\hline $04 / 20 / 2002$ & - & - & 0.20 & E0.01 & E0.01 & - & E0.01 & 0.15 \\
\hline $05 / 14 / 2002$ & - & - & 0.20 & E0.01 & E0.01 & - & - & E0.02 \\
\hline $05 / 26 / 2002$ & - & - & 0.16 & - & - & - & - & E0.01 \\
\hline 06/07/2002 & - & - & 0.46 & E0.01 & E0.01 & - & - & 0.20 \\
\hline 06/19/2002 & - & - & 0.44 & E0.01 & E0.01 & - & - & E0.08 \\
\hline $07 / 01 / 2002$ & - & - & 0.71 & E0.04 & E0.03 & - & - & 0.28 \\
\hline $07 / 13 / 2002$ & - & - & 0.49 & E0.03 & E0.02 & - & E0.02 & E0.12 \\
\hline $07 / 25 / 2002$ & - & - & 0.32 & E0.01 & E0.01 & - & E0.03 & E0.12 \\
\hline 08/06/2002 & - & - & 0.39 & E0.02 & E0.01 & - & E0.03 & E0.11 \\
\hline $08 / 18 / 2002$ & - & - & 0.14 & E0.01 & E0.01 & - & E0.02 & E0.03 \\
\hline 08/30/2002 & E0.01 & - & 0.83 & E0.03 & E0.02 & - & E0.01 & E0.09 \\
\hline $09 / 11 / 2002$ & NA & NA & NA & NA & NA & NA & NA & NA \\
\hline $09 / 23 / 2002$ & NA & NA & NA & NA & NA & NA & NA & NA \\
\hline $10 / 05 / 2002$ & - & - & 0.76 & E0.02 & E0.01 & - & E0.05 & 0.15 \\
\hline $10 / 17 / 2002$ & - & - & 0.17 & E0.01 & E0.01 & - & - & 0.42 \\
\hline $10 / 29 / 2002$ & - & - & 0.63 & E0.02 & E0.01 & - & E0.08 & 0.37 \\
\hline $11 / 10 / 2002$ & - & - & 1.76 & 0.06 & E0.05 & - & - & E0.10 \\
\hline $11 / 22 / 2002$ & - & - & 0.91 & E0.03 & E0.02 & - & E0.02 & E0.12 \\
\hline $12 / 04 / 2002$ & - & - & 0.25 & E0.01 & E0.01 & - & - & E0.05 \\
\hline $01 / 20 / 2003$ & - & - & 0.53 & E0.02 & E0.01 & - & E0.03 & E0.03 \\
\hline $02 / 04 / 2003$ & - & - & E0.03 & E0.01 & E0.01 & - & E0.02 & E0.05 \\
\hline $02 / 14 / 2003$ & - & - & 0.19 & E0.01 & E0.01 & - & E0.01 & E0.05 \\
\hline $02 / 26 / 2003$ & - & - & 0.68 & E0.02 & E0.01 & - & E0.02 & E0.04 \\
\hline 03/10/2003 & - & - & 0.35 & E0.01 & E0.01 & - & E0.02 & E0.07 \\
\hline $03 / 22 / 2003$ & - & - & 0.53 & E0.01 & E0.01 & - & E0.01 & - \\
\hline $04 / 03 / 2003$ & - & - & 0.27 & E0.01 & E0.01 & - & E0.02 & 0.14 \\
\hline
\end{tabular}


Table 9B. Analytical results for volatile organic compounds in air with high breakthrough volumes from the Sweetwater Reservoir atmospheric site, San Diego County, California.-Continued

[The site identification number is 324141117001601 . Concentrations are given in parts per billion by volume (ppbv). E, estimated value; NA, not analyzed; - , compound not detected at a concentration above the laboratory reporting level]

\begin{tabular}{|c|c|c|c|c|c|c|c|c|}
\hline $\begin{array}{c}\text { Date } \\
\text { (mm/dd/yyyy) }\end{array}$ & $\begin{array}{c}\text { Tetra- } \\
\text { hydrofuran }\end{array}$ & $\begin{array}{l}\text { 1,2,-Dibromo- } \\
\text { 3-chloro- } \\
\text { propene } \\
\text { (DBCP) }\end{array}$ & $\begin{array}{c}m \text { - and } p-- \\
\text { Xylene }\end{array}$ & $\begin{array}{c}\text { 1,2,3,5- } \\
\text { Tetra- } \\
\text { methyl- } \\
\text { benzene }\end{array}$ & $\begin{array}{c}\text { 1,2,4,5- } \\
\text { Tetra- } \\
\text { methyl- } \\
\text { benzene }\end{array}$ & $\begin{array}{l}\text { Methyl- } \\
\text { acetate }\end{array}$ & $\begin{array}{l}\text { tert-Amyl } \\
\text { alcohol }\end{array}$ & $\begin{array}{l}\text { tert-Butyl } \\
\text { alcohol }\end{array}$ \\
\hline $04 / 15 / 2003$ & - & - & 0.28 & E0.01 & E0.01 & - & E0.02 & E0.05 \\
\hline 05/09/2003 & - & - & 0.15 & E0.01 & E0.01 & - & E0.01 & E0.08 \\
\hline $05 / 21 / 2003$ & - & - & 1.03 & E0.02 & E0.01 & - & 0.16 & 0.14 \\
\hline 06/02/2003 & - & - & 0.33 & E0.01 & E0.01 & - & E0.02 & 0.33 \\
\hline $06 / 14 / 2003$ & - & - & 0.16 & E0.01 & E0.01 & - & E0.02 & E0.13 \\
\hline $06 / 26 / 2003$ & - & - & 0.53 & E0.01 & E0.01 & - & E0.02 & E0.10 \\
\hline 07/09/2003 & NA & NA & NA & NA & NA & NA & NA & NA \\
\hline $07 / 20 / 2003$ & - & - & 0.29 & E0.02 & E0.02 & - & E0.07 & 0.27 \\
\hline 08/01/2003 & - & - & 0.36 & E0.02 & E0.01 & - & E0.03 & 0.29 \\
\hline 08/13/2003 & - & - & 0.52 & E0.02 & E0.01 & - & E0.03 & E0.09 \\
\hline $08 / 25 / 2003$ & - & - & 0.41 & E0.03 & E0.02 & - & E0.05 & E0.09 \\
\hline 09/06/2003 & E0.01 & - & 0.83 & E0.03 & E0.02 & - & E0.02 & E0.10 \\
\hline $09 / 18 / 2003$ & - & - & 0.79 & E0.02 & E0.01 & - & E0.05 & 0.14 \\
\hline
\end{tabular}


Table 10A. Polycyclic aromatic hydrocarbon (PAH) and alkylated PAH compounds analyzed in air samples collected from the Sweetwater Reservoir atmospheric site, San Diego County, California.

[The site identification number is 324141117001601 . Estimated reporting level for split extract analyzed in full scan mode assumes 315 cubic meters as the average air volume. Alkylated PAH homolog concentrations are estimated. $\mathrm{ng} / \mathrm{m}^{3}$, nanogram per cubic meter]

\begin{tabular}{|c|c|c|}
\hline Compound & $\begin{array}{c}\text { Estimated } \\
\text { reporting } \\
\text { level }^{1} \\
\left(\mathrm{ng} / \mathrm{m}^{3}\right)\end{array}$ & $\begin{array}{c}\text { Ion } \\
\text { (mass-to- } \\
\text { charge ratio) } \\
\text { monitored for } \\
\text { alkylated PAH } \\
\text { homologs }\end{array}$ \\
\hline Anthracene & 0.5 & \\
\hline $\operatorname{Benz}[a]$ anthracene & 0.5 & \\
\hline Benzo $[g h i]$ perylene & 0.5 & \\
\hline Benzo $[a]$ pyrene & 0.5 & \\
\hline Benzo $[b]$ fluoranthene & 0.5 & \\
\hline Benzo $[e]$ pyrene & 0.5 & \\
\hline Benzo $[k]$ fluoranthene & 0.5 & \\
\hline Chrysene & 0.5 & \\
\hline Coronene & 0.5 & \\
\hline Dibenzo $[a, h]$ anthracene & 0.5 & \\
\hline Fluoranthene & 0.5 & \\
\hline Indeno $[1,2,3-c d]$ pyrene & 0.5 & \\
\hline 2-Methylanthracene & 0.5 & \\
\hline 4,5-Methylenephenanthrene & 0.5 & \\
\hline 1-Methylphenanthrene & 0.5 & \\
\hline 1-Methylpyrene & 0.5 & \\
\hline Perylene & 0.5 & \\
\hline Phenanthrene & 0.5 & \\
\hline Pyrene & 0.5 & \\
\hline \multicolumn{3}{|c|}{ Alkylated PAH homologs } \\
\hline C1-178 Isomers, methylated phenanthrenes/anthracenes & 0.5 & 178 \\
\hline C1-202 Isomers, methylated fluoranthenes/pyrenes & 0.5 & 202 \\
\hline C1-228 Isomers, methylated benzo $[a]$ anthracenes/chrysenes & 0.5 & 228 \\
\hline C1-252 Isomers, methylated benzopyrenes/perylenes & 0.5 & 252 \\
\hline C2-178 Isomers, alkylated phenanthrenes/anthracenes & 0.5 & 178 \\
\hline C2-202 Isomers, alkylated fluoranthenes/pyrenes & 0.5 & 202 \\
\hline C2-228 Isomers, alkylated benzo[ $a]$ anthracenes/chrysenes & 0.5 & 228 \\
\hline C2-252 Isomers, alkylated benzopyrenes/perylenes & 0.5 & 252 \\
\hline C3-178 Isomers, alkylated phenanthrenes/anthracenes & 0.5 & 178 \\
\hline C3-202 Isomers, alkylated fluoranthenes/pyrenes & 0.5 & 202 \\
\hline C3-228 Isomers, alkylated benzo[ $a$ ]anthracenes/chrysenes & 0.5 & 228 \\
\hline C3-252 Isomers, alkylated benzopyrenes/perylenes & 0.5 & 252 \\
\hline C4-178 Isomers, alkylated phenanthrenes/anthracenes & 0.5 & 178 \\
\hline C4-202 Isomers, alkylated fluoranthenes/pyrenes & 0.5 & 202 \\
\hline C4-228 Isomers, alkylated benzo[ $a]$ anthracenes/chrysenes & 0.5 & 228 \\
\hline C4-252 Isomers, alkylated benzopyrenes/perylenes & 0.5 & 252 \\
\hline C5-178 Isomers, alkylated phenanthrenes/anthracenes & 0.5 & 178 \\
\hline C5-202 Isomers, alkylated fluoranthenes/pyrenes & 0.5 & 202 \\
\hline C5-228 Isomers, alkylated benzo[ $a]$ anthracenes/chrysenes & 0.5 & 228 \\
\hline C5-252 Isomers, alkylated benzopyrenes/perylenes & 0.5 & 252 \\
\hline 2-Fluorobiphenyl, surrogate & percent & \\
\hline Nitrobenzene- $d 5$, surrogate & percent & \\
\hline Terphenyl- $d 14$, surrogate & percent & \\
\hline
\end{tabular}


Table 10B. Analytical results for polycyclic aromatic hydrocarbon (PAH) and alkylated PAH concentrations in air from the Sweetwater Reservoir atmospheric site, San Diego County, California.

[The site identification number is 324141117001601 . Values are given in nanograms per cubic meter $\left(\mathrm{ng} / \mathrm{m}^{3}\right)$ unless noted. Concentrations of individual PAH that are less than $0.5 \mathrm{ng} / \mathrm{m}^{3}$ (the reporting level) are estimated. $\mu \mathrm{g} / \mathrm{m}^{3}$, microgram per cubic meter; E, estimated concentration; GCMS, gas chromatography mass spectrometry; GFF, glass fiber filter; $\mathrm{m}^{3}$, cubic meter; PSE, pressurized solvent extraction; PUF, polyurethane foam; Scan, full scan ion monitoring mode; SIM, selected ion monitoring mode; PSE, pressurized solvent extraction; - , compound not detected at a concentration above the laboratory reporting level]

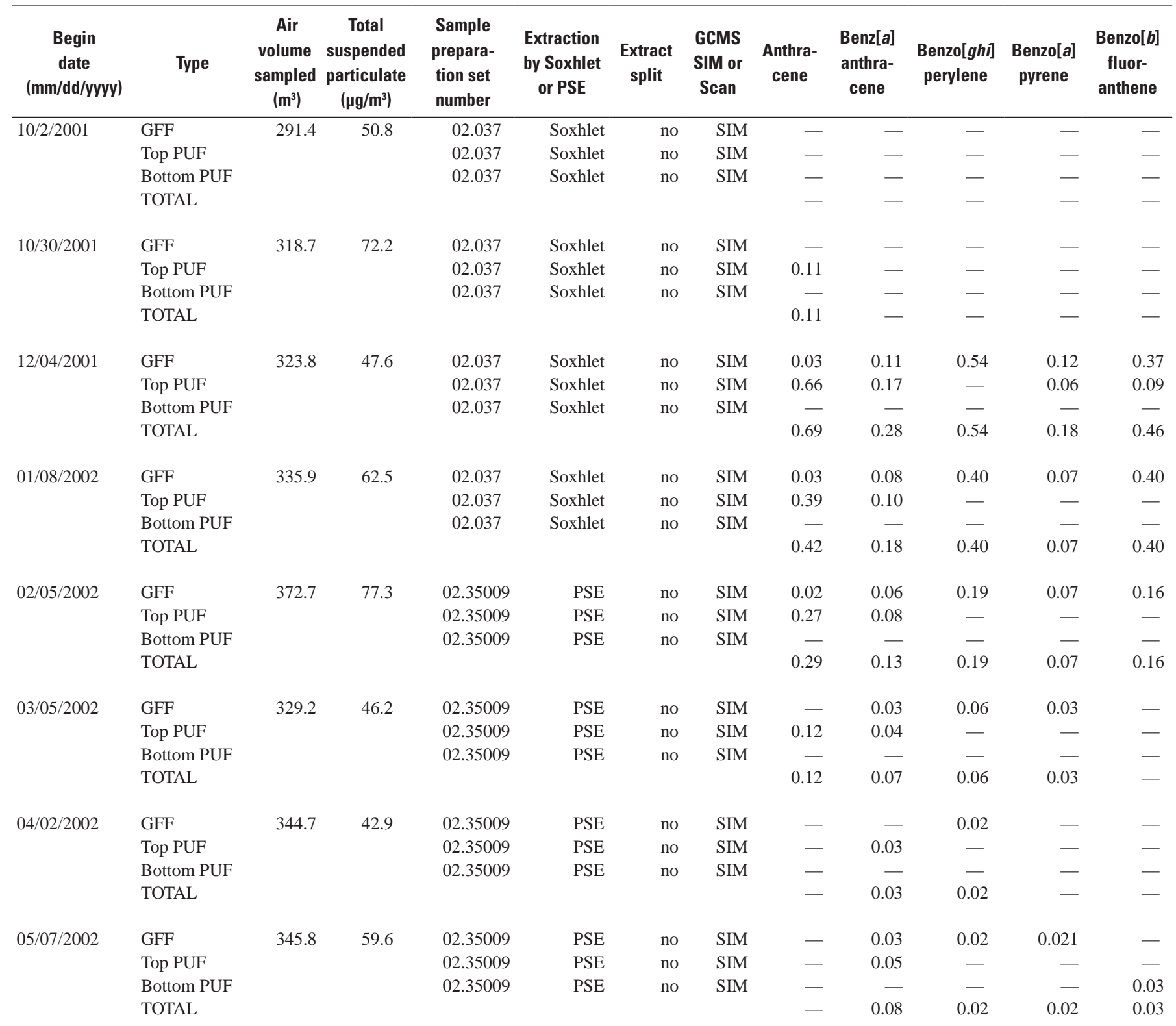


Table 10B. Analytical results for polycyclic aromatic hydrocarbon (PAH) and alkylated PAH concentrations in air from the Sweetwater Reservoir atmospheric site, San Diego County, California.-Continued

[The site identification number is 324141117001601 . Values are given in nanograms per cubic meter $\left(\mathrm{ng} / \mathrm{m}^{3}\right)$, unless noted. Concentrations of individual PAH that are less than $0.5 \mathrm{ng} / \mathrm{m}^{3}$ (the reporting level) are estimated. $\mu \mathrm{g} / \mathrm{m}^{3}$, microgram per cubic meter; E, estimated concentration; GCMS, gas chromatography mass spectrometry; GFF, glass fiber filter; $\mathrm{m}^{3}$, cubic meter; PSE, pressurized solvent extraction; PUF, polyurethane foam; Scan, full scan ion monitoring mode; SIM, selected ion monitoring mode; PSE, pressurized solvent extraction; - , compound not detected at a concentration above the laboratory reporting level]

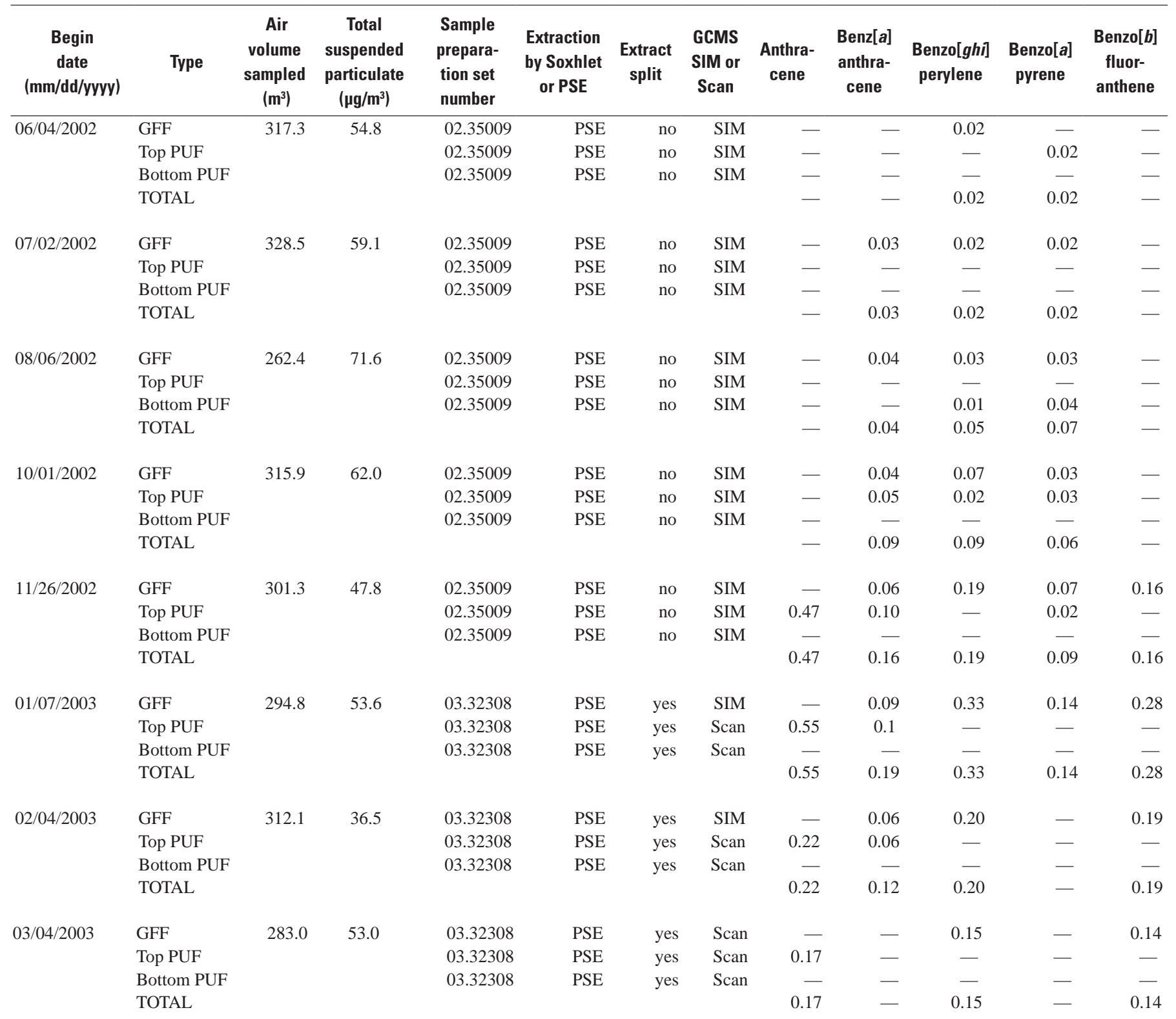


Table 10B. Analytical results for polycyclic aromatic hydrocarbon (PAH) and alkylated PAH concentrations in air from the Sweetwater Reservoir atmospheric site, San Diego County, California.-Continued

[The site identification number is 324141117001601 . Values are given in nanograms per cubic meter $\left(\mathrm{ng} / \mathrm{m}^{3}\right)$ unless noted. Concentrations of individual PAH that are less than $0.5 \mathrm{ng} / \mathrm{m}^{3}$ (the reporting level) are estimated. $\mu \mathrm{g} / \mathrm{m}^{3}$, microgram per cubic meter; E, estimated concentration; GCMS, gas chromatography mass spectrometry; GFF, glass fiber filter; $\mathrm{m}^{3}$, cubic meter; PSE, pressurized solvent extraction; PUF, polyurethane foam; Scan, full scan ion monitoring mode; SIM, selected ion monitoring mode; PSE, pressurized solvent extraction; - , compound not detected at a concentration above the laboratory reporting level]

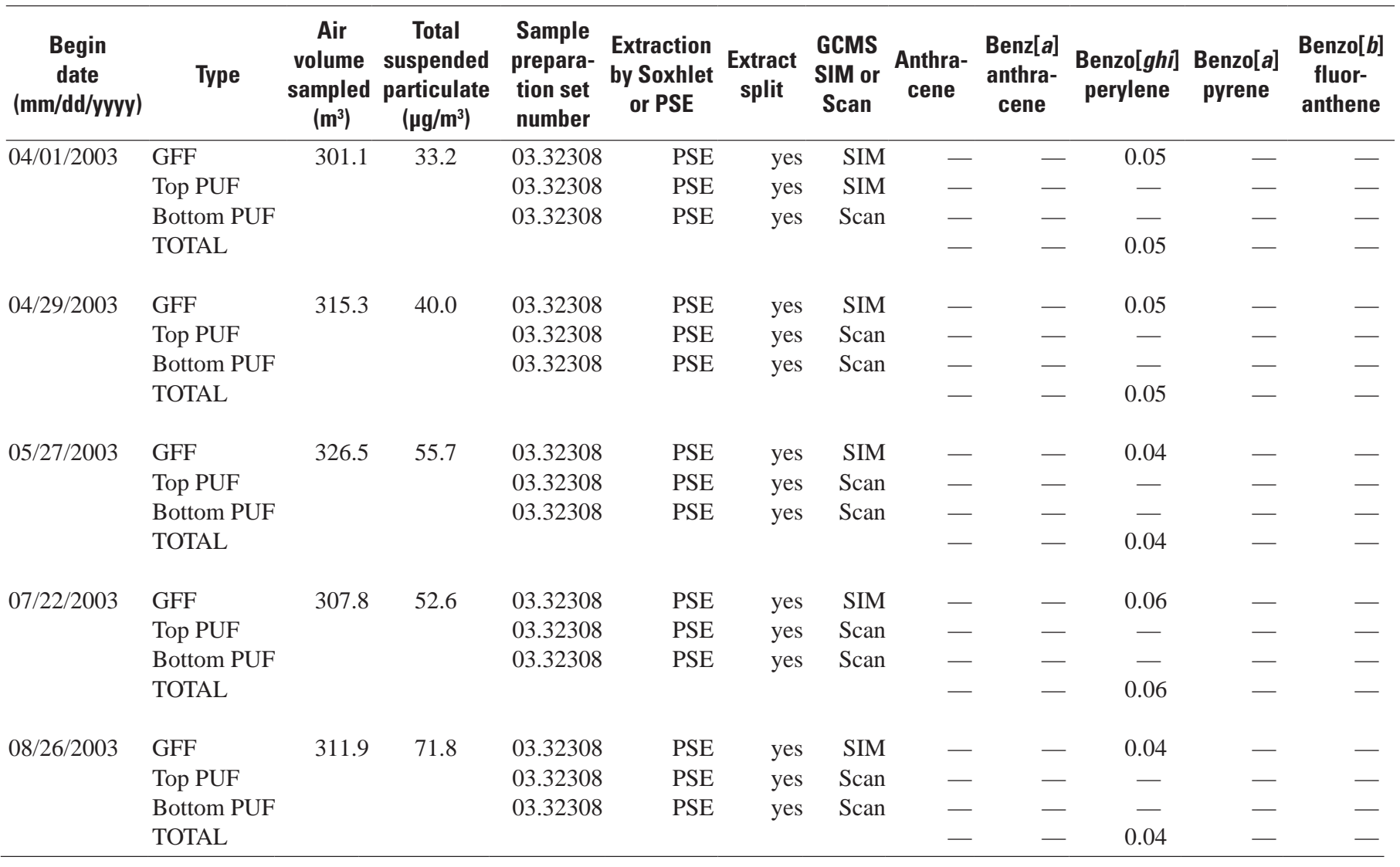


Table 10B. Analytical results for polycyclic aromatic hydrocarbon (PAH) and alkylated PAH concentrations in air from the Sweetwater Reservoir atmospheric site, San Diego County, California.-Continued

[The site identification number is 324141117001601 . Values are given in nanograms per cubic meter $\left(\mathrm{ng} / \mathrm{m}^{3}\right)$, unless noted. Concentrations of individual PAH that are less than $0.5 \mathrm{ng} / \mathrm{m}^{3}$ (the reporting level) are estimated. $\mu \mathrm{g} / \mathrm{m}^{3}$, microgram per cubic meter; E, estimated concentration; GCMS,

gas chromatography mass spectrometry; GFF, glass fiber filter; $\mathrm{m}^{3}$, cubic meter; PSE, pressurized solvent extraction; PUF, polyurethane foam; Scan, full scan ion monitoring mode; SIM, selected ion monitoring mode; PSE, pressurized solvent extraction; - , compound not detected at a concentration above the laboratory reporting level]

\begin{tabular}{|c|c|c|c|c|c|c|c|c|c|c|c|c|}
\hline $\begin{array}{c}\text { Begin } \\
\text { date } \\
\text { (mm/dd/yyyy) }\end{array}$ & Type & $\begin{array}{c}\text { Air } \\
\text { volume } \\
\text { sampled } \\
\left(\mathbf{m}^{3}\right)\end{array}$ & $\begin{array}{c}\text { Total } \\
\text { suspended } \\
\text { particulate } \\
\left(\mu \mathrm{g} / \mathrm{m}^{3}\right)\end{array}$ & $\begin{array}{c}\text { Sample } \\
\text { prepara- } \\
\text { tion set } \\
\text { number }\end{array}$ & $\begin{array}{l}\text { Extraction } \\
\text { by Soxhlet } \\
\text { or PSE }\end{array}$ & $\begin{array}{l}\text { Extract } \\
\text { split }\end{array}$ & $\begin{array}{l}\text { GCMS } \\
\text { SIM or } \\
\text { Scan }\end{array}$ & $\begin{array}{c}\text { Benzo[e] } \\
\text { pyrene }\end{array}$ & $\begin{array}{l}\text { Benzo[k] } \\
\text { fluor- } \\
\text { anthene }\end{array}$ & $\begin{array}{l}\text { Chry- } \\
\text { sene }\end{array}$ & Coronene & $\begin{array}{c}\text { Dibenzo } \\
{[a, h]} \\
\text { anthra- } \\
\text { cene }\end{array}$ \\
\hline \multirow[t]{4}{*}{$10 / 2 / 2001$} & GFF & 291.4 & 50.8 & 02.037 & Soxhlet & no & SIM & - & - & 0.02 & - & - \\
\hline & Top PUF & & & 02.037 & Soxhlet & no & SIM & - & - & 0.03 & - & - \\
\hline & Bottom PUF & & & 02.037 & Soxhlet & no & SIM & - & - & - & - & - \\
\hline & TOTAL & & & & & & & - & - & 0.05 & - & - \\
\hline \multirow[t]{4}{*}{$10 / 30 / 2001$} & GFF & 318.7 & 72.2 & 02.037 & Soxhlet & no & SIM & - & - & 0.02 & - & - \\
\hline & Top PUF & & & 02.037 & Soxhlet & no & SIM & - & - & 0.16 & - & - \\
\hline & Bottom PUF & & & 02.037 & Soxhlet & no & SIM & - & - & - & - & - \\
\hline & TOTAL & & & & & & & - & - & 0.18 & - & - \\
\hline \multirow[t]{4}{*}{$12 / 04 / 2001$} & GFF & 323.8 & 47.6 & 02.037 & Soxhlet & no & SIM & 0.34 & 0.40 & 0.18 & 0.35 & - \\
\hline & Top PUF & & & 02.037 & Soxhlet & no & SIM & 0.04 & 0.08 & 0.35 & - & - \\
\hline & Bottom PUF & & & 02.037 & Soxhlet & no & SIM & - & - & - & - & - \\
\hline & TOTAL & & & & & & & 0.38 & 0.48 & 0.53 & 0.35 & - \\
\hline \multirow[t]{4}{*}{ 01/08/2002 } & GFF & 335.9 & 62.5 & 02.037 & Soxhlet & no & SIM & 0.29 & 0.36 & 0.17 & 0.28 & - \\
\hline & Top PUF & & & 02.037 & Soxhlet & no & SIM & - & - & 0.27 & - & - \\
\hline & Bottom PUF & & & 02.037 & Soxhlet & no & SIM & - & - & - & - & - \\
\hline & TOTAL & & & & & & & 0.29 & 0.36 & 0.44 & 0.28 & - \\
\hline \multirow[t]{4}{*}{$02 / 05 / 2002$} & GFF & 372.7 & 77.3 & 02.35009 & PSE & no & SIM & 0.11 & 0.11 & 0.07 & 0.14 & 0.02 \\
\hline & Top PUF & & & 02.35009 & PSE & no & SIM & 0.03 & - & 0.19 & - & - \\
\hline & Bottom PUF & & & 02.35009 & PSE & no & SIM & - & - & - & - & - \\
\hline & TOTAL & & & & & & & 0.14 & 0.11 & 0.26 & 0.14 & 0.02 \\
\hline \multirow[t]{4}{*}{ 03/05/2002 } & GFF & 329.2 & 46.2 & 02.35009 & PSE & no & SIM & 0.05 & - & 0.04 & 0.05 & - \\
\hline & Top PUF & & & 02.35009 & PSE & no & SIM & - & - & 0.10 & - & - \\
\hline & Bottom PUF & & & 02.35009 & PSE & no & SIM & - & - & - & - & - \\
\hline & TOTAL & & & & & & & 0.05 & - & 0.14 & 0.05 & - \\
\hline \multirow[t]{4}{*}{ 04/02/2002 } & GFF & 344.7 & 42.9 & 02.35009 & PSE & no & SIM & - & - & - & 0.02 & - \\
\hline & Top PUF & & & 02.35009 & PSE & no & SIM & 0.01 & - & 0.04 & - & - \\
\hline & Bottom PUF & & & 02.35009 & PSE & no & SIM & - & - & - & - & - \\
\hline & TOTAL & & & & & & & 0.01 & - & 0.04 & 0.02 & - \\
\hline \multirow[t]{4}{*}{ 05/07/2002 } & GFF & 345.8 & 59.6 & 02.35009 & PSE & no & SIM & 0.02 & - & 0.02 & 0.02 & - \\
\hline & Top PUF & & & 02.35009 & PSE & no & SIM & - & - & 0.04 & - & - \\
\hline & Bottom PUF & & & 02.35009 & PSE & no & SIM & - & - & - & - & - \\
\hline & TOTAL & & & & & & & 0.02 & - & 0.06 & 0.02 & - \\
\hline
\end{tabular}


Table 10B. Analytical results for polycyclic aromatic hydrocarbon (PAH) and alkylated PAH concentrations in air from the Sweetwater Reservoir atmospheric site, San Diego County, California.-Continued

[The site identification number is 324141117001601 . Values are given in nanograms per cubic meter $\left(\mathrm{ng} / \mathrm{m}^{3}\right)$ unless noted. Concentrations of individual PAH that are less than $0.5 \mathrm{ng} / \mathrm{m}^{3}$ (the reporting level) are estimated. $\mu \mathrm{g} / \mathrm{m}^{3}$, microgram per cubic meter; E, estimated concentration; GCMS, gas chromatography mass spectrometry; GFF, glass fiber filter; $\mathrm{m}^{3}$, cubic meter; PSE, pressurized solvent extraction; PUF, polyurethane foam; Scan, full scan ion monitoring mode; SIM, selected ion monitoring mode; PSE, pressurized solvent extraction; - , compound not detected at a concentration above the laboratory reporting level]

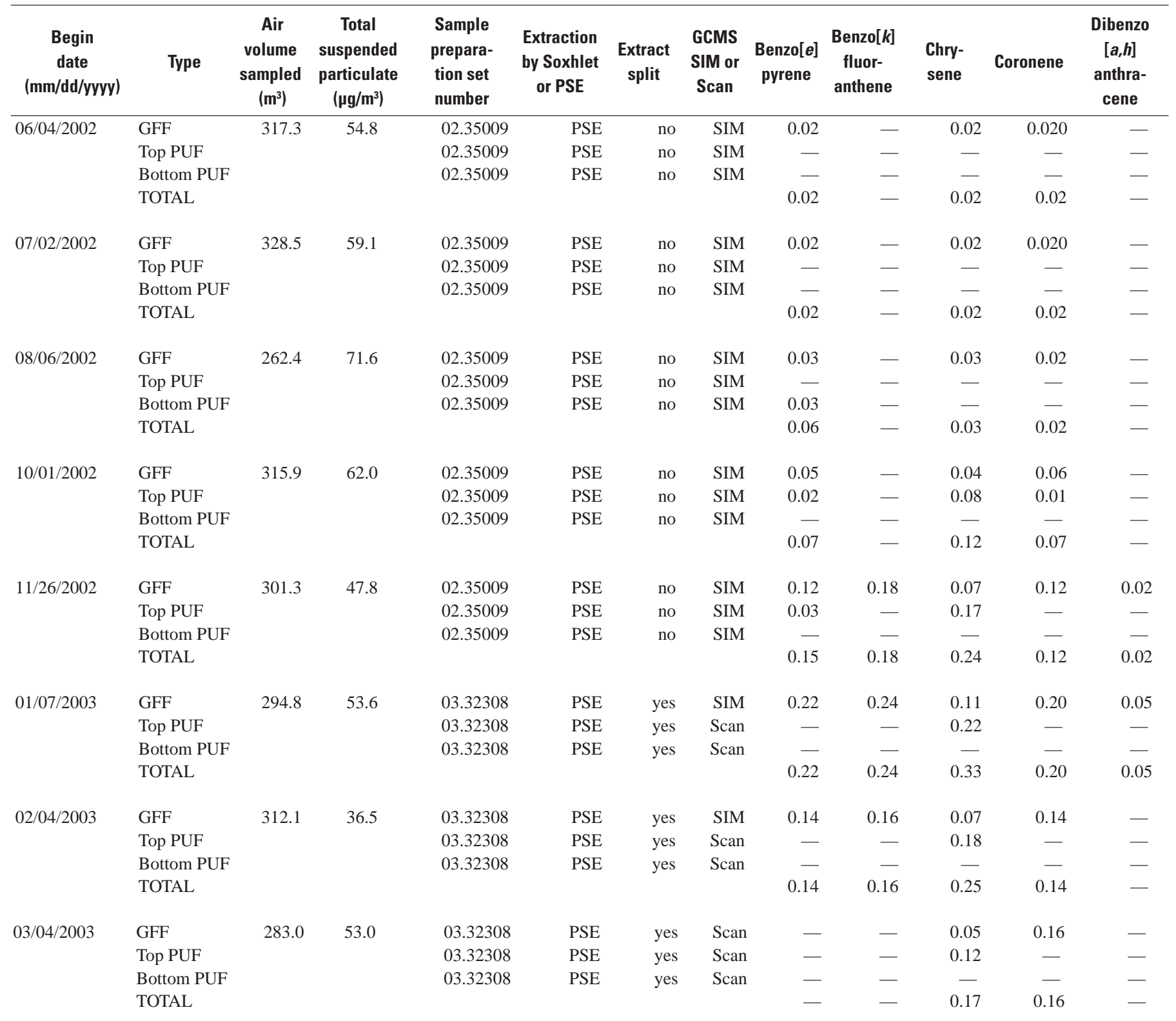


Table 10B. Analytical results for polycyclic aromatic hydrocarbon (PAH) and alkylated PAH concentrations in air from the Sweetwater Reservoir atmospheric site, San Diego County, California.-Continued

[The site identification number is 324141117001601 . Values are given in nanograms per cubic meter $\left(\mathrm{ng} / \mathrm{m}^{3}\right)$ unless noted. Concentrations of individual PAH that are less than $0.5 \mathrm{ng} / \mathrm{m}^{3}$ (the reporting level) are estimated. $\mu \mathrm{g} / \mathrm{m}^{3}$, microgram per cubic meter; E, estimated concentration; GCMS, gas chromatography mass spectrometry; GFF, glass fiber filter; $\mathrm{m}^{3}$, cubic meter; PSE, pressurized solvent extraction; PUF, polyurethane foam; Scan, full scan ion monitoring mode; SIM, selected ion monitoring mode; PSE, pressurized solvent extraction; - , compound not detected at a concentration above the laboratory reporting level]

\begin{tabular}{|c|c|c|c|c|c|c|c|c|c|c|c|c|}
\hline $\begin{array}{c}\text { Begin } \\
\text { date } \\
\text { (mm/dd/yyyy) }\end{array}$ & Type & $\begin{array}{c}\text { Air } \\
\text { volume } \\
\text { sampled } \\
\left(\mathbf{m}^{3}\right)\end{array}$ & $\begin{array}{c}\text { Total } \\
\text { suspended } \\
\text { particulate } \\
\left(\mu \mathrm{g} / \mathrm{m}^{3}\right)\end{array}$ & $\begin{array}{c}\text { Sample } \\
\text { prepara- } \\
\text { tion set } \\
\text { number }\end{array}$ & $\begin{array}{c}\text { Extraction by } \\
\text { Soxhlet } \\
\text { or PSE }\end{array}$ & $\begin{array}{c}\text { Extract } \\
\text { split }\end{array}$ & $\begin{array}{l}\text { GCMS } \\
\text { SIM or } \\
\text { Scan }\end{array}$ & $\begin{array}{c}\text { Benzo[e] } \\
\text { pyrene }\end{array}$ & $\begin{array}{l}\text { Benzo[ } k] \\
\text { fluor- } \\
\text { anthene }\end{array}$ & $\begin{array}{l}\text { Chry- } \\
\text { sene }\end{array}$ & Coronene & $\begin{array}{c}\text { Dibenzo } \\
{[a, h]} \\
\text { anthra- } \\
\text { cene }\end{array}$ \\
\hline \multirow[t]{3}{*}{$04 / 01 / 2003$} & GFF & 301.1 & 33.2 & 03.32308 & PSE & yes & SIM & - & - & - & 0.04 & - \\
\hline & Top PUF & & & 03.32308 & PSE & yes & SIM & - & - & 0.02 & - & - \\
\hline & $\begin{array}{c}\text { Bottom } \\
\text { PUF }\end{array}$ & & & 03.32308 & PSE & yes & Scan & - & - & - & - & - \\
\hline \multirow[t]{4}{*}{$04 / 29 / 2003$} & GFF & 315.3 & 40.0 & 03.32308 & PSE & yes & SIM & - & - & - & 0.04 & - \\
\hline & Top PUF & & & 03.32308 & PSE & yes & Scan & - & - & - & - & - \\
\hline & $\begin{array}{r}\text { Bottom } \\
\text { PUF }\end{array}$ & & & 03.32308 & PSE & yes & Scan & - & - & - & - & - \\
\hline & TOTAL & & & & & & & - & - & - & 0.04 & - \\
\hline $05 / 27 / 2003$ & TOTAL & & & & & & & - & - & - & 0.04 & - \\
\hline \multirow[t]{5}{*}{$07 / 22 / 2003$} & GFF & 307.8 & 52.6 & 03.32308 & PSE & yes & SIM & - & - & - & 0.06 & - \\
\hline & Top PUF & & & 03.32308 & PSE & yes & Scan & - & - & - & - & - \\
\hline & Bottom & & & 03.32308 & PSE & yes & Scan & - & - & - & - & - \\
\hline & PUF & & & & & & & & & & & \\
\hline & TOTAL & & & & & & & - & - & - & 0.06 & - \\
\hline \multirow[t]{3}{*}{$08 / 26 / 2003$} & GFF & 311.9 & 71.8 & 03.32308 & PSE & yes & SIM & - & - & - & 0.04 & - \\
\hline & Top PUF & & & 03.32308 & PSE & yes & Scan & - & - & - & - & - \\
\hline & Bottom & & & 03.32308 & PSE & yes & Scan & - & - & - & - & - \\
\hline
\end{tabular}


Table 10B. Analytical results for polycyclic aromatic hydrocarbon (PAH) and alkylated PAH concentrations in air from the Sweetwater Reservoir atmospheric site, San Diego County, California.-Continued

[The site identification number is 324141117001601 . Values are given in nanograms per cubic meter $\left(\mathrm{ng} / \mathrm{m}^{3}\right)$ unless noted. Concentrations of individual PAH that are less than $0.5 \mathrm{ng} / \mathrm{m}^{3}$ (the reporting level) are estimated. $\mu \mathrm{g} / \mathrm{m}^{3}$, microgram per cubic meter; E, estimated concentration; GCMS, gas chromatography mass spectrometry; GFF, glass fiber filter; $\mathrm{m}^{3}$, cubic meter; PSE, pressurized solvent extraction; PUF, polyurethane foam; Scan, full scan ion monitoring mode; SIM, selected ion monitoring mode; PSE, pressurized solvent extraction; - , compound not detected at a concentration above the laboratory reporting level]

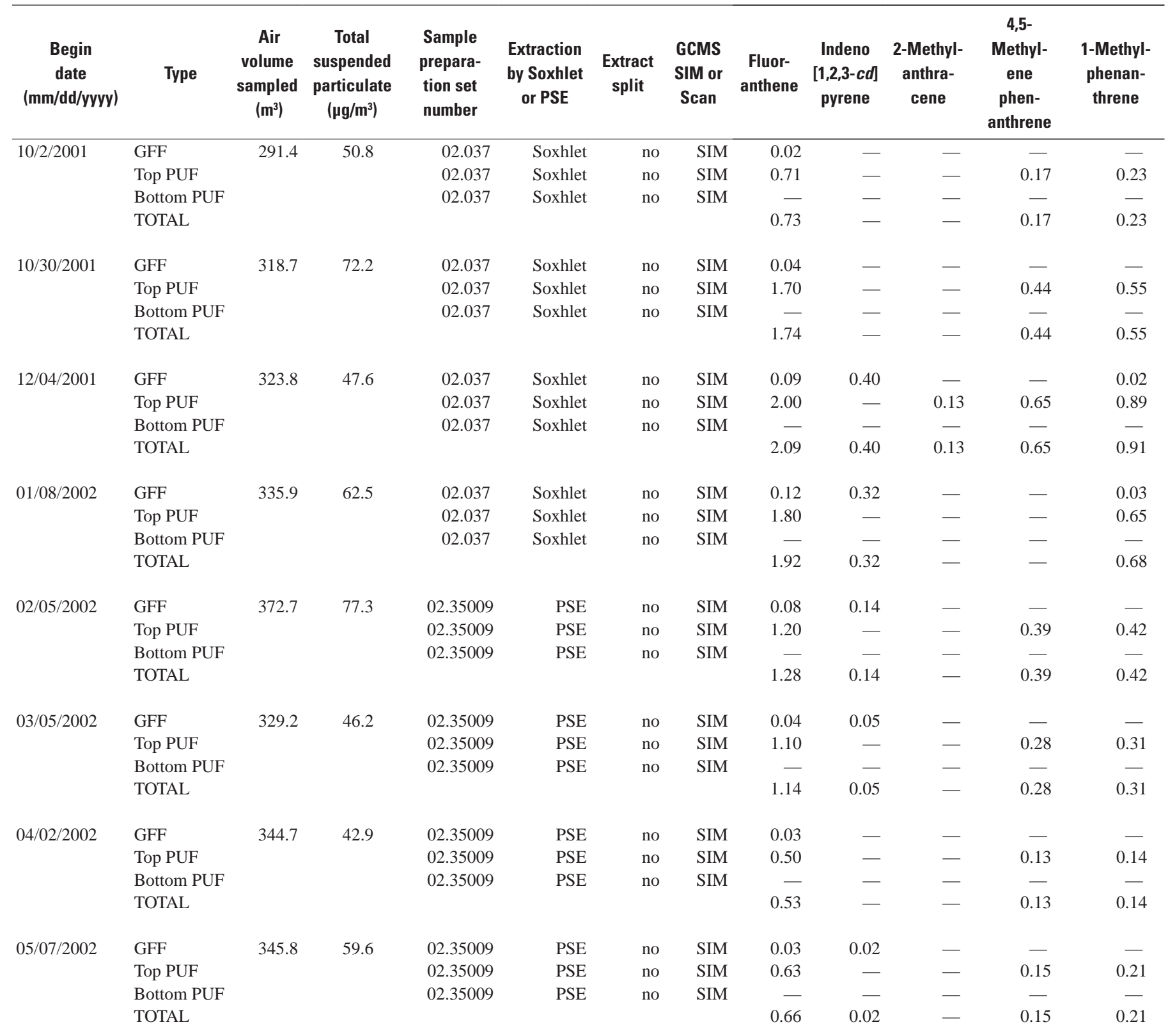


Table 10B. Analytical results for polycyclic aromatic hydrocarbon (PAH) and alkylated PAH concentrations in air from the Sweetwater Reservoir atmospheric site, San Diego County, California.-Continued

[The site identification number is 324141117001601 . Values are given in nanograms per cubic meter $\left(\mathrm{ng} / \mathrm{m}^{3}\right)$ unless noted. Concentrations of individual PAH that are less than $0.5 \mathrm{ng} / \mathrm{m}^{3}$ (the reporting level) are estimated. $\mu \mathrm{g} / \mathrm{m}^{3}$, microgram per cubic meter; E, estimated concentration; GCMS, gas chromatography mass spectrometry; GFF, glass fiber filter; $\mathrm{m}^{3}$, cubic meter; PSE, pressurized solvent extraction; PUF, polyurethane foam; Scan, full scan ion monitoring mode; SIM, selected ion monitoring mode; PSE, pressurized solvent extraction; - , compound not detected at a concentration above the laboratory reporting level]

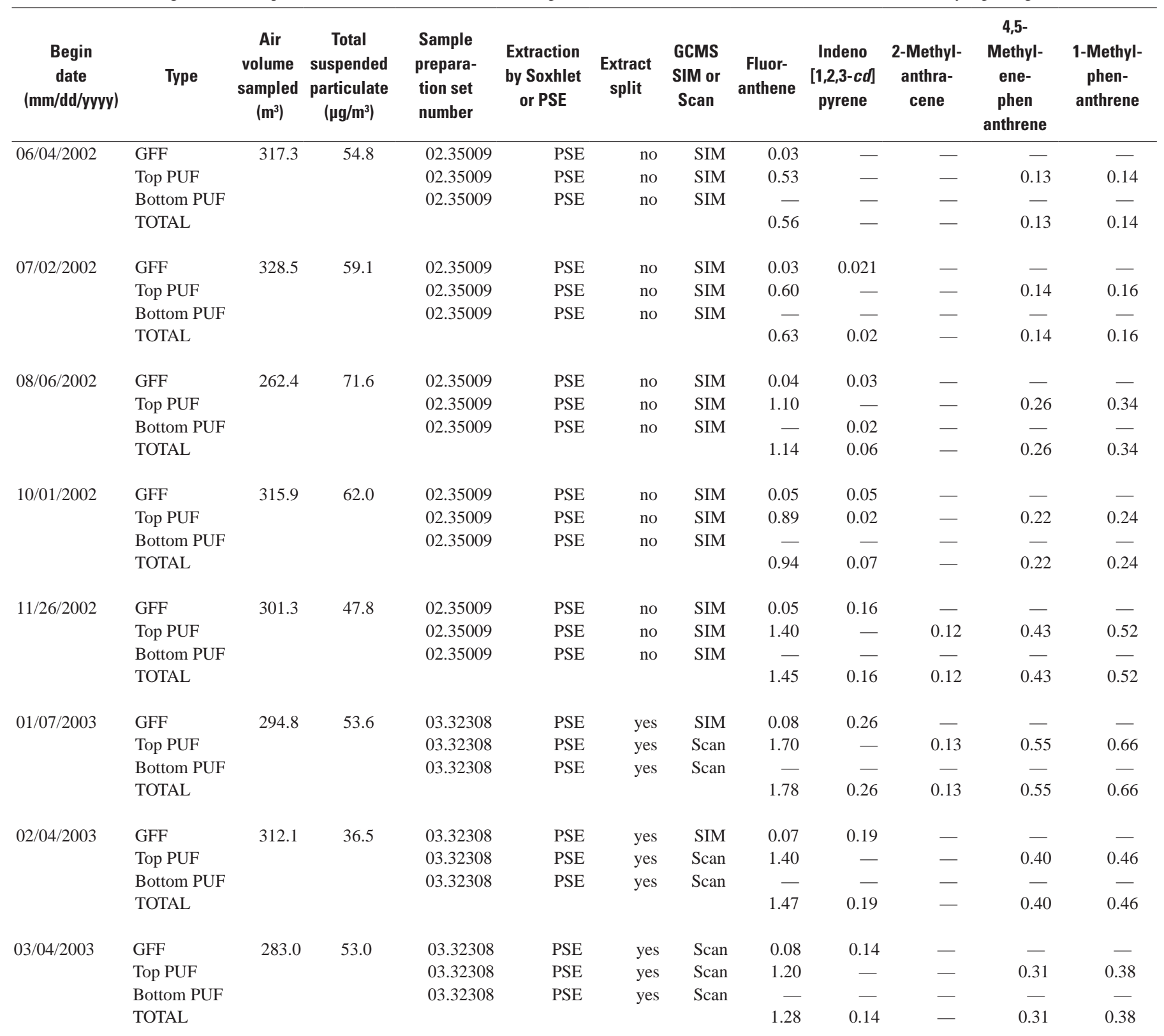


Table 10B. Analytical results for polycyclic aromatic hydrocarbon (PAH) and alkylated PAH concentrations in air from the Sweetwater Reservoir atmospheric site, San Diego County, California.-Continued

[The site identification number is 324141117001601 . Values are given in nanograms per cubic meter $\left(\mathrm{ng} / \mathrm{m}^{3}\right)$ unless noted. Concentrations of individual PAH that are less than $0.5 \mathrm{ng} / \mathrm{m}^{3}$ (the reporting level) are estimated. $\mu \mathrm{g} / \mathrm{m}^{3}$, microgram per cubic meter; E, estimated concentration; GCMS, gas chromatography mass spectrometry; GFF, glass fiber filter; $\mathrm{m}^{3}$, cubic meter; PSE, pressurized solvent extraction; PUF, polyurethane foam; Scan, full scan ion monitoring mode; SIM, selected ion monitoring mode; PSE, pressurized solvent extraction; - , compound not detected at a concentration above the laboratory reporting level]

\begin{tabular}{|c|c|c|c|c|c|c|c|c|c|c|c|c|}
\hline $\begin{array}{c}\text { Begin } \\
\text { date } \\
\text { (mm/dd/yyyy) }\end{array}$ & Type & $\begin{array}{c}\text { Air } \\
\text { volume } \\
\text { sampled } \\
\left(\mathbf{m}^{3}\right)\end{array}$ & $\begin{array}{c}\text { Total } \\
\text { suspended } \\
\text { particulate } \\
\left(\mu \mathrm{g} / \mathrm{m}^{3}\right)\end{array}$ & $\begin{array}{c}\text { Sample } \\
\text { prepara- } \\
\text { tion set } \\
\text { number }\end{array}$ & $\begin{array}{l}\text { Extraction } \\
\text { by Soxhlet } \\
\text { or PSE }\end{array}$ & $\begin{array}{c}\text { Extract } \\
\text { split }\end{array}$ & $\begin{array}{l}\text { GCMS } \\
\text { SIM or } \\
\text { Scan }\end{array}$ & $\begin{array}{c}\text { Fluor- } \\
\text { anthene }\end{array}$ & $\begin{array}{c}\text { Indeno } \\
{[1,2,3-c d]} \\
\text { pyrene }\end{array}$ & $\begin{array}{c}\text { 2-Methyl- } \\
\text { anthra- } \\
\text { cene }\end{array}$ & $\begin{array}{c}\text { 4,5- } \\
\text { Methyl- } \\
\text { ene- } \\
\text { phen } \\
\text { anthrene }\end{array}$ & $\begin{array}{c}\text { 1-Methyl- } \\
\text { phenan- } \\
\text { threne }\end{array}$ \\
\hline \multirow[t]{3}{*}{$04 / 01 / 2003$} & GFF & 301.1 & 33.2 & 03.32308 & PSE & yes & SIM & - & - & - & - & - \\
\hline & Top PUF & & & 03.32308 & PSE & yes & SIM & 0.22 & - & - & - & 0.11 \\
\hline & Bottom PUF & & & 03.32308 & PSE & yes & Scan & - & - & - & - & - \\
\hline \multirow[t]{4}{*}{ 04/29/2003 } & GFF & 315.3 & 40.0 & 03.32308 & PSE & yes & SIM & - & - & - & - & - \\
\hline & Top PUF & & & 03.32308 & PSE & yes & Scan & 0.64 & - & - & 0.16 & 0.17 \\
\hline & Bottom PUF & & & 03.32308 & PSE & yes & Scan & - & - & - & - & - \\
\hline & TOTAL & & & & & & & 0.64 & - & - & 0.16 & 0.17 \\
\hline $05 / 27 / 2003$ & GFF & 326.5 & 55.7 & 03.32308 & PSE & yes & SIM & 0.05 & - & - & - & - \\
\hline \multirow[t]{4}{*}{$07 / 22 / 2003$} & GFF & 307.8 & 52.6 & 03.32308 & PSE & yes & SIM & 0.05 & - & - & - & 0.04 \\
\hline & Top PUF & & & 03.32308 & PSE & yes & Scan & 0.92 & - & - & 0.20 & 0.30 \\
\hline & Bottom PUF & & & 03.32308 & PSE & yes & Scan & - & - & - & - & - \\
\hline & TOTAL & & & & & & & 0.97 & - & - & 0.20 & 0.34 \\
\hline \multirow[t]{4}{*}{$08 / 26 / 2003$} & GFF & 311.9 & 71.8 & 03.32308 & PSE & yes & SIM & 0.05 & - & - & - & - \\
\hline & Top PUF & & & 03.32308 & PSE & yes & Scan & 1.20 & - & - & 0.22 & 0.33 \\
\hline & Bottom PUF & & & 03.32308 & PSE & yes & Scan & - & - & - & - & - \\
\hline & TOTAL & & & & & & & 1.25 & - & - & 0.22 & 0.33 \\
\hline
\end{tabular}


Table 10B. Analytical results for polycyclic aromatic hydrocarbon (PAH) and alkylated PAH concentrations in air from the Sweetwater Reservoir atmospheric site, San Diego County, California.-Continued

[The site identification number is 324141117001601 . Values are given in nanograms per cubic meter $\left(\mathrm{ng} / \mathrm{m}^{3}\right)$ unless noted. Concentrations of individual PAH that are less than $0.5 \mathrm{ng} / \mathrm{m}^{3}$ (the reporting level) are estimated. $\mu \mathrm{g} / \mathrm{m}^{3}$, microgram per cubic meter; E, estimated concentration; GCMS, gas chromatography mass spectrometry; GFF, glass fiber filter; $\mathrm{m}^{3}$, cubic meter; PSE, pressurized solvent extraction; PUF, polyurethane foam; Scan, full scan ion monitoring mode; SIM, selected ion monitoring mode; PSE, pressurized solvent extraction; - , compound not detected at a concentration above the laboratory reporting level]

\begin{tabular}{|c|c|c|c|c|c|c|c|c|c|c|c|c|}
\hline $\begin{array}{c}\text { Begin } \\
\text { date } \\
\text { (mm/dd/yyyy) }\end{array}$ & Type & $\begin{array}{c}\text { Air } \\
\text { volume } \\
\text { sampled } \\
\left(\mathbf{m}^{3}\right)\end{array}$ & $\begin{array}{c}\text { Total } \\
\text { suspended } \\
\text { particulate } \\
\left(\mu \mathrm{g} / \mathrm{m}^{3}\right)\end{array}$ & $\begin{array}{c}\text { Sample } \\
\text { preparat- } \\
\text { ion set } \\
\text { number }\end{array}$ & $\begin{array}{l}\text { Extraction } \\
\text { by Soxhlet } \\
\text { or PSE }\end{array}$ & $\begin{array}{l}\text { Extract } \\
\text { split }\end{array}$ & $\begin{array}{l}\text { GCMS } \\
\text { SIM or } \\
\text { Scan }\end{array}$ & $\begin{array}{c}1- \\
\text { Methyl- } \\
\text { pyrene }\end{array}$ & Perylene & $\begin{array}{c}\text { Phen- } \\
\text { anthrene }\end{array}$ & Pyrene & $\begin{array}{c}\text { C1-178 } \\
\text { Isomers, } \\
\text { methylated } \\
\text { phen- } \\
\text { anthrenes/ } \\
\text { anthracenes } \\
\text { (E) }\end{array}$ \\
\hline \multirow{2}{*}{$10 / 2 / 2001$} & Bottom PUF & & & 02.037 & Soxhlet & no & SIM & - & - & 0.96 & - & - \\
\hline & TOTAL & & & & & & & 0.02 & - & 3.80 & 0.40 & 2.80 \\
\hline \multirow[t]{3}{*}{ 10/30/2001 } & GFF & 318.7 & 72.2 & 02.037 & Soxhlet & no & SIM & - & - & 0.03 & 0.04 & - \\
\hline & Top PUF & & & 02.037 & Soxhlet & no & SIM & 0.06 & - & 5.80 & 1.10 & 7.30 \\
\hline & Bottom PUF & & & 02.037 & Soxhlet & no & SIM & - & - & 2.50 & - & - \\
\hline \multirow{2}{*}{$12 / 04 / 2001$} & Bottom PUF & & & 02.037 & Soxhlet & no & SIM & 0.01 & - & 0.30 & - & - \\
\hline & TOTAL & & & & & & & 0.18 & - & 8.85 & 1.81 & 7.10 \\
\hline \multirow[t]{4}{*}{ 01/08/2002 } & GFF & 335.9 & 62.5 & 02.037 & Soxhlet & no & SIM & - & - & 0.06 & 0.12 & 0.08 \\
\hline & Top PUF & & & 02.037 & Soxhlet & no & SIM & 0.09 & - & 7.30 & 1.50 & 13.0 \\
\hline & Bottom PUF & & & 02.037 & Soxhlet & no & SIM & - & - & 0.51 & - & - \\
\hline & TOTAL & & & & & & & 0.09 & - & 7.87 & 1.62 & 13.1 \\
\hline \multirow[t]{4}{*}{$02 / 05 / 2002$} & GFF & 372.7 & 77.3 & 02.35009 & PSE & no & SIM & 0.02 & 0.02 & 0.04 & 0.09 & 0.06 \\
\hline & Top PUF & & & 02.35009 & PSE & no & SIM & 0.07 & - & 4.80 & 1.10 & 5.00 \\
\hline & Bottom PUF & & & 02.35009 & PSE & no & SIM & - & - & 0.37 & - & 1.30 \\
\hline & TOTAL & & & & & & & 0.09 & 0.02 & 5.21 & 1.19 & 6.36 \\
\hline 04/02/2002 & TOTAL & & & & & & & 0.03 & - & 2.33 & 0.36 & 3.54 \\
\hline \multirow[t]{4}{*}{ 05/07/2002 } & GFF & 345.8 & 59.6 & 02.35009 & PSE & no & SIM & - & - & - & 0.03 & - \\
\hline & Top PUF & & & 02.35009 & PSE & no & SIM & - & - & 3.10 & 0.31 & 5.10 \\
\hline & Bottom PUF & & & 02.35009 & PSE & no & SIM & - & - & 0.29 & - & 0.15 \\
\hline & TOTAL & & & & & & & - & - & 3.39 & 0.34 & 5.25 \\
\hline
\end{tabular}


Table 10B. Analytical results for polycyclic aromatic hydrocarbon (PAH) and alkylated PAH concentrations in air from the Sweetwater Reservoir atmospheric site, San Diego County, California.-Continued

[The site identification number is 324141117001601 . Values are given in nanograms per cubic meter $\left(\mathrm{ng} / \mathrm{m}^{3}\right)$ unless noted. Concentrations of individual PAH that are less than $0.5 \mathrm{ng} / \mathrm{m}^{3}$ (the reporting level) are estimated. $\mu \mathrm{g} / \mathrm{m}^{3}$, microgram per cubic meter; E, estimated concentration; GCMS, gas chromatography mass spectrometry; GFF, glass fiber filter; $\mathrm{m}^{3}$, cubic meter; PSE, pressurized solvent extraction; PUF, polyurethane foam; Scan, full scan ion monitoring mode; SIM, selected ion monitoring mode; PSE, pressurized solvent extraction; - , compound not detected at a concentration above the laboratory reporting level]

\begin{tabular}{|c|c|c|c|c|c|c|c|c|c|c|c|c|}
\hline $\begin{array}{c}\text { Begin } \\
\text { date } \\
\text { (mm/dd/yyyy) }\end{array}$ & Type & $\begin{array}{c}\text { Air } \\
\text { volume } \\
\text { sampled } \\
\left(\mathbf{m}^{3}\right)\end{array}$ & $\begin{array}{c}\text { Total } \\
\text { suspended } \\
\text { particulate } \\
\left(\mu \mathrm{g} / \mathrm{m}^{3}\right)\end{array}$ & $\begin{array}{c}\text { Sample } \\
\text { prepara- } \\
\text { tion set } \\
\text { number }\end{array}$ & $\begin{array}{l}\text { Extraction } \\
\text { by Soxhlet } \\
\text { or PSE }\end{array}$ & $\begin{array}{l}\text { Extract } \\
\text { split }\end{array}$ & $\begin{array}{l}\text { GCMS } \\
\text { SIM or } \\
\text { Scan }\end{array}$ & $\begin{array}{c}\text { 1- } \\
\text { Methyl- } \\
\text { pyrene }\end{array}$ & Perylene & $\begin{array}{c}\text { Phen- } \\
\text { anthrene }\end{array}$ & Pyrene & $\begin{array}{c}\text { C1-178 } \\
\text { Isomers, } \\
\text { methylated } \\
\text { phen- } \\
\text { anthrenes/ } \\
\text { anthracenes } \\
\text { (E) }\end{array}$ \\
\hline \multirow{2}{*}{ 06/04/2002 } & Bottom PUF & & & 02.35009 & PSE & no & SIM & - & - & 0.33 & - & - \\
\hline & TOTAL & & & & & & & - & - & 2.43 & 0.32 & 1.90 \\
\hline \multirow[t]{3}{*}{ 07/02/2002 } & GFF & 328.5 & 59.1 & 02.35009 & PSE & no & SIM & - & - & 0.02 & 0.03 & - \\
\hline & Top PUF & & & 02.35009 & PSE & no & SIM & - & - & 2.41 & 0.31 & 3.48 \\
\hline & Bottom PUF & & & 02.35009 & PSE & no & SIM & - & - & 0.64 & - & - \\
\hline \multirow{2}{*}{ 08/06/2002 } & Bottom PUF & & & 02.35009 & PSE & no & SIM & - & - & 0.91 & - & - \\
\hline & TOTAL & & & & & & & - & - & 6.14 & 0.61 & 2.50 \\
\hline \multirow[t]{4}{*}{$10 / 01 / 2002$} & GFF & 315.9 & 62.0 & 02.35009 & PSE & no & SIM & - & - & 0.03 & 0.06 & - \\
\hline & Top PUF & & & 02.35009 & PSE & no & SIM & 0.04 & - & 4.30 & 0.56 & 2.80 \\
\hline & Bottom PUF & & & 02.35009 & PSE & no & SIM & - & - & 0.50 & - & - \\
\hline & TOTAL & & & & & & & 0.04 & - & 4.83 & 0.62 & 2.80 \\
\hline \multirow[t]{4}{*}{$11 / 26 / 2002$} & GFF & 301.3 & 47.8 & 02.35009 & PSE & no & SIM & 0.03 & 0.02 & 0.03 & 0.05 & - \\
\hline & Top PUF & & & 02.35009 & PSE & no & SIM & 0.09 & - & 5.90 & 1.10 & 2.80 \\
\hline & Bottom PUF & & & 02.35009 & PSE & no & SIM & 0.02 & - & 0.04 & - & - \\
\hline & TOTAL & & & & & & & 0.14 & 0.02 & 5.97 & 1.15 & 2.80 \\
\hline $02 / 04 / 2003$ & TOTAL & & & & & & & - & - & 6.26 & 1.18 & 2.10 \\
\hline \multirow[t]{4}{*}{ 03/04/2003 } & GFF & 283.0 & 53.0 & 03.32308 & PSE & yes & Scan & - & - & 0.05 & 0.09 & - \\
\hline & Top PUF & & & 03.32308 & PSE & yes & Scan & - & - & 6.00 & 0.81 & 1.90 \\
\hline & Bottom PUF & & & 03.32308 & PSE & yes & Scan & - & - & 0.37 & - & - \\
\hline & TOTAL & & & & & & & - & - & 6.42 & 0.90 & 1.90 \\
\hline
\end{tabular}


Table 10B. Analytical results for polycyclic aromatic hydrocarbon (PAH) and alkylated PAH concentrations in air from the Sweetwater Reservoir atmospheric site, San Diego County, California.-Continued

[The site identification number is 324141117001601 . Values are given in nanograms per cubic meter $\left(\mathrm{ng} / \mathrm{m}^{3}\right)$ unless noted. Concentrations of individual PAH that are less than $0.5 \mathrm{ng} / \mathrm{m}^{3}$ (the reporting level) are estimated. $\mu \mathrm{g} / \mathrm{m}^{3}$, microgram per cubic meter; E, estimated concentration; GCMS, gas chromatography mass spectrometry; GFF, glass fiber filter; $\mathrm{m}^{3}$, cubic meter; PSE, pressurized solvent extraction; PUF, polyurethane foam; Scan, full scan ion monitoring mode; SIM, selected ion monitoring mode; PSE, pressurized solvent extraction; - , compound not detected at a concentration above the laboratory reporting level]

\begin{tabular}{|c|c|c|c|c|c|c|c|c|c|c|c|c|}
\hline $\begin{array}{c}\text { Begin } \\
\text { date } \\
\text { (mm/dd/yyyy) }\end{array}$ & Type & $\begin{array}{c}\text { Air } \\
\text { volume } \\
\text { sampled } \\
\left(\mathrm{m}^{3}\right)\end{array}$ & $\begin{array}{c}\text { Total } \\
\text { suspended } \\
\text { particulate } \\
\left(\mu \mathrm{g} / \mathrm{m}^{3}\right)\end{array}$ & $\begin{array}{c}\text { Sample } \\
\text { prepara- } \\
\text { tion set } \\
\text { number }\end{array}$ & $\begin{array}{c}\text { Extraction } \\
\text { by Soxhlet } \\
\text { or PSE }\end{array}$ & $\begin{array}{c}\text { Extract } \\
\text { split }\end{array}$ & $\begin{array}{l}\text { GCMS } \\
\text { SIM or } \\
\text { Scan }\end{array}$ & $\begin{array}{c}\text { 1- } \\
\text { Methyl- } \\
\text { pyrene }\end{array}$ & Perylene & $\begin{array}{c}\text { Phen- } \\
\text { anthrene }\end{array}$ & Pyrene & $\begin{array}{c}\text { C1-178 } \\
\text { Isomers, } \\
\text { methylated } \\
\text { phen- } \\
\text { anthrenes/ } \\
\text { anthracenes } \\
\text { (E) }\end{array}$ \\
\hline \multirow{2}{*}{$04 / 01 / 2003$} & Bottom PUF & & & 03.32308 & PSE & yes & Scan & - & - & 0.17 & - & - \\
\hline & TOTAL & & & & & & & - & - & 1.37 & 0.14 & 0.59 \\
\hline \multirow[t]{3}{*}{$04 / 29 / 2003$} & GFF & 315.3 & 40.0 & 03.32308 & PSE & yes & SIM & - & - & - & - & - \\
\hline & Top PUF & & & 03.32308 & PSE & yes & Scan & - & - & 2.90 & 0.42 & 0.98 \\
\hline & Bottom PUF & & & 03.32308 & PSE & yes & Scan & - & - & 0.14 & - & - \\
\hline $05 / 27 / 2003$ & TOTAL & & & & & & & - & - & 3.60 & 0.45 & 1.10 \\
\hline \multirow[t]{4}{*}{$07 / 22 / 2003$} & GFF & 307.8 & 52.6 & 03.32308 & PSE & yes & SIM & - & - & 0.05 & 0.05 & - \\
\hline & Top PUF & & & 03.32308 & PSE & yes & Scan & - & - & 3.30 & 0.57 & 1.70 \\
\hline & Bottom PUF & & & 03.32308 & PSE & yes & Scan & - & - & 1.90 & - & - \\
\hline & TOTAL & & & & & & & - & - & 5.25 & 0.62 & 1.70 \\
\hline \multirow[t]{4}{*}{$08 / 26 / 2003$} & GFF & 311.9 & 71.8 & 03.32308 & PSE & yes & SIM & - & - & 0.04 & 0.05 & - \\
\hline & Top PUF & & & 03.32308 & PSE & yes & Scan & - & - & 2.90 & 0.60 & 1.80 \\
\hline & Bottom PUF & & & 03.32308 & PSE & yes & Scan & - & - & 3.30 & - & - \\
\hline & TOTAL & & & & & & & - & - & 6.24 & 0.65 & 1.80 \\
\hline
\end{tabular}


Table 10B. Analytical results for polycyclic aromatic hydrocarbon (PAH) and alkylated PAH concentrations in air from the Sweetwater Reservoir atmospheric site, San Diego County, California.-Continued

[The site identification number is 324141117001601 . Values are given in nanograms per cubic meter $\left(\mathrm{ng} / \mathrm{m}^{3}\right)$ unless noted. Concentrations of individual PAH that are less than $0.5 \mathrm{ng} / \mathrm{m}^{3}$ (the reporting level) are estimated. $\mu \mathrm{g} / \mathrm{m}^{3}$, microgram per cubic meter; E, estimated concentration; GCMS, gas chromatography mass spectrometry; GFF, glass fiber filter; $\mathrm{m}^{3}$, cubic meter; PSE, pressurized solvent extraction; PUF, polyurethane foam; Scan, full scan ion monitoring mode; SIM, selected ion monitoring mode; PSE, pressurized solvent extraction; —, compound not detected at a concentration above the laboratory reporting level]

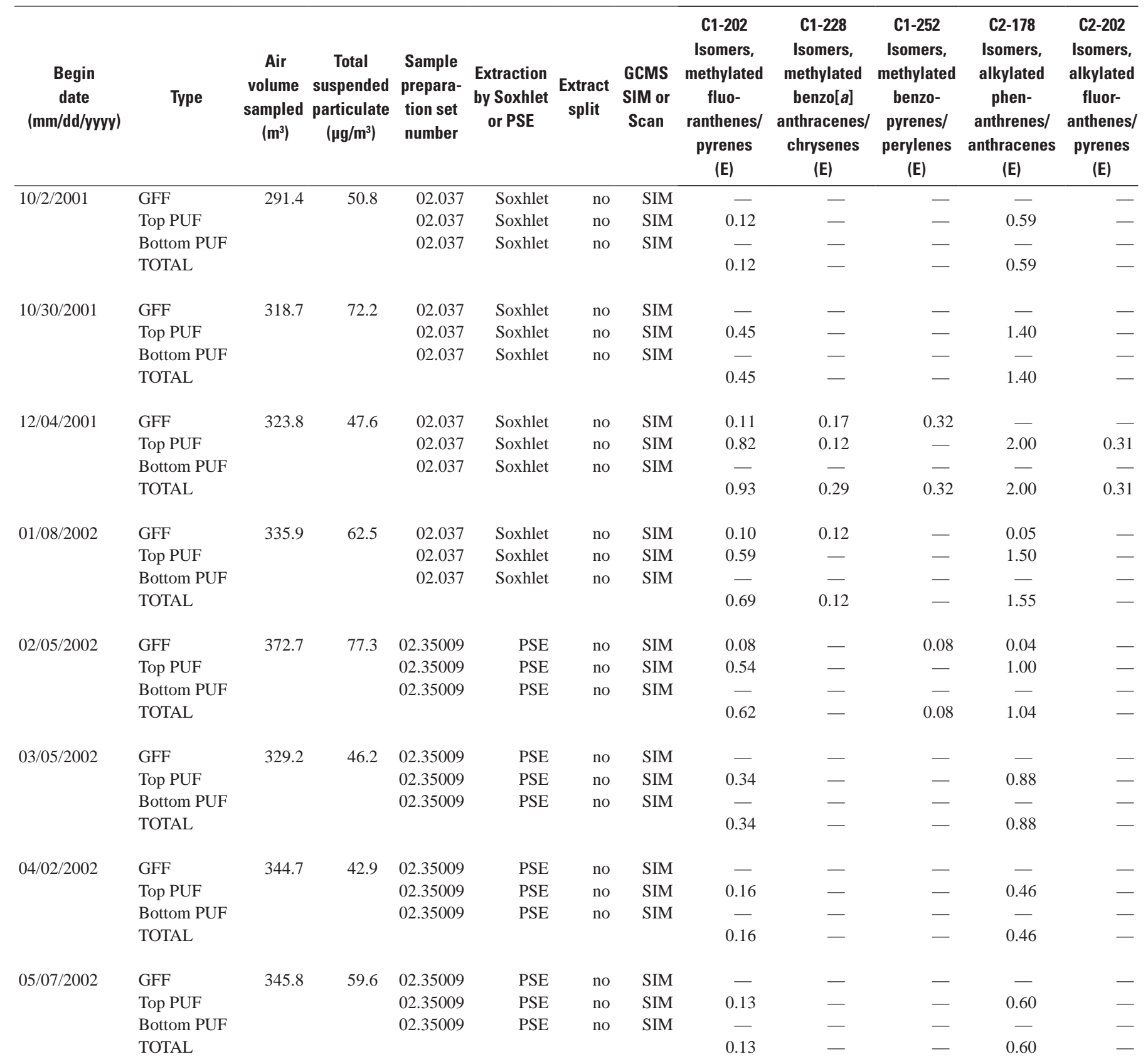


Table 10B. Analytical results for polycyclic aromatic hydrocarbon (PAH) and alkylated PAH concentrations in air from the Sweetwater Reservoir atmospheric site, San Diego County, California.-Continued

[The site identification number is 324141117001601 . Values are given in nanograms per cubic meter $\left(\mathrm{ng} / \mathrm{m}^{3}\right)$ unless noted. Concentrations of individual PAH that are less than $0.5 \mathrm{ng} / \mathrm{m}^{3}$ (the reporting level) are estimated. $\mu \mathrm{g} / \mathrm{m}^{3}$, microgram per cubic meter; E, estimated concentration; GCMS, gas chromatography mass spectrometry; GFF, glass fiber filter; $\mathrm{m}^{3}$, cubic meter; PSE, pressurized solvent extraction; PUF, polyurethane foam; Scan, full scan ion monitoring mode; SIM, selected ion monitoring mode; PSE, pressurized solvent extraction; - , compound was not detected at a concentration above the laboratory reporting level]

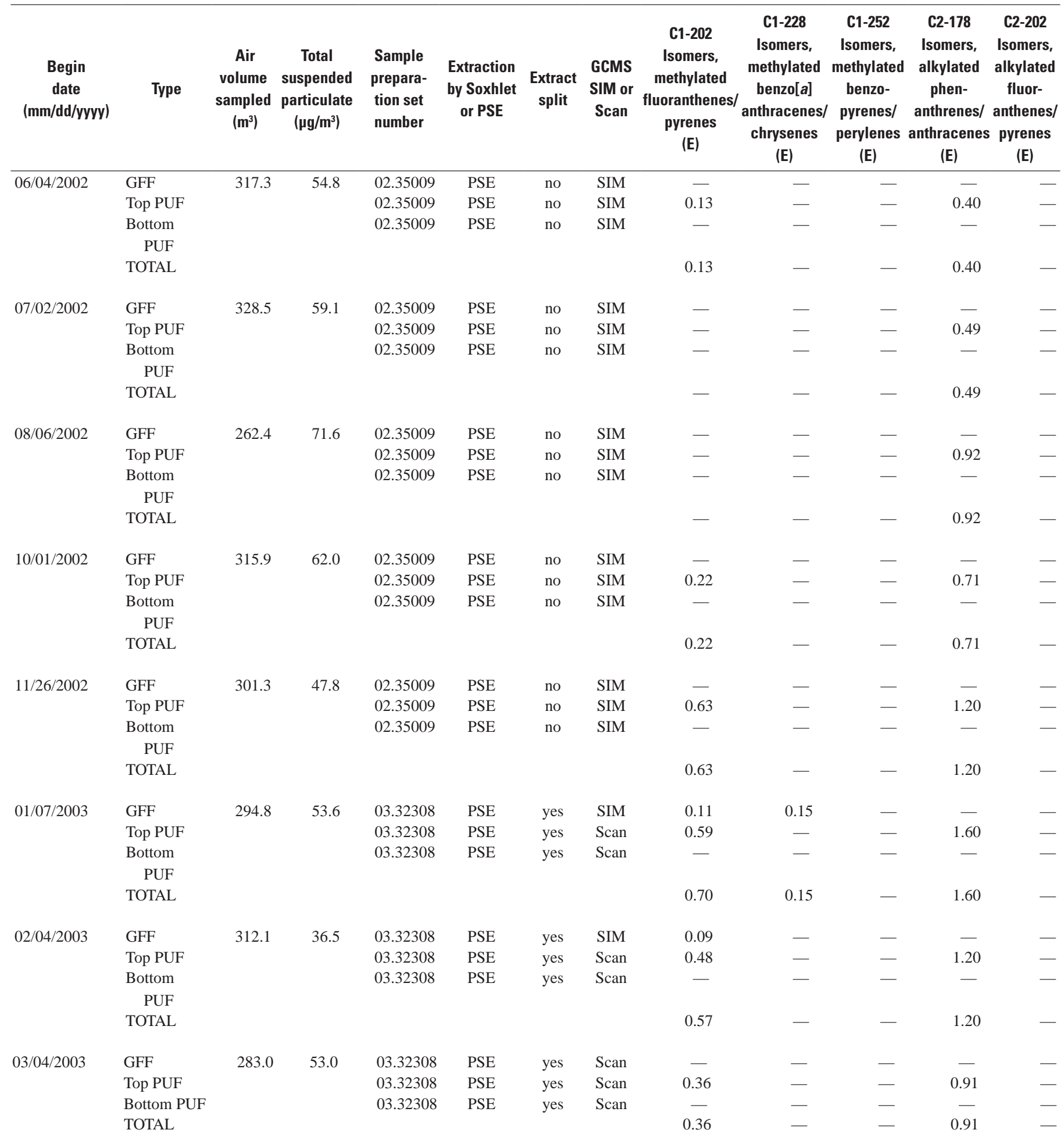


Table 10B. Analytical results for polycyclic aromatic hydrocarbon (PAH) and alkylated PAH concentrations in air from the Sweetwater Reservoir atmospheric site, San Diego County, California.-Continued

[The site identification number is 324141117001601 . Values are given in nanograms per cubic meter $\left(\mathrm{ng} / \mathrm{m}^{3}\right)$ unless noted. Concentrations of individual PAH that are less than $0.5 \mathrm{ng} / \mathrm{m}^{3}$ (the reporting level) are estimated. $\mu \mathrm{g} / \mathrm{m}^{3}$, microgram per cubic meter; E, estimated concentration; GCMS, gas chromatography mass spectrometry; GFF, glass fiber filter; $\mathrm{m}^{3}$, cubic meter; PSE, pressurized solvent extraction; PUF, polyurethane foam; Scan, full scan ion monitoring mode; SIM, selected ion monitoring mode; PSE, pressurized solvent extraction; —, compound was not detected at a concentration above the laboratory reporting level]

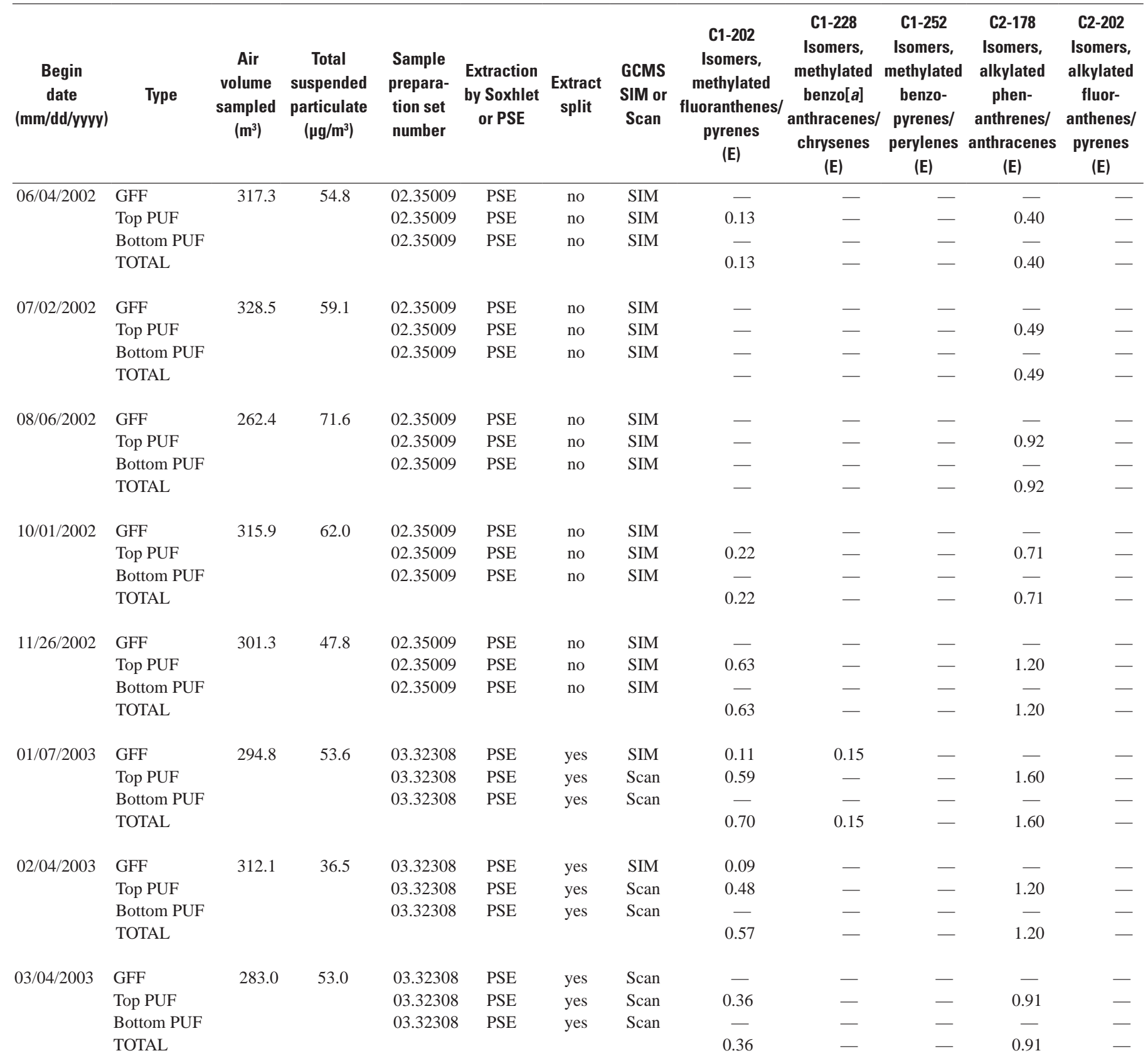


Table 10B. Analytical results for polycyclic aromatic hydrocarbon (PAH) and alkylated PAH concentrations in air from the Sweetwater Reservoir atmospheric site, San Diego County, California.-Continued

[The site identification number is 324141117001601 . Values are given in nanograms per cubic meter $\left(\mathrm{ng} / \mathrm{m}^{3}\right)$ unless noted. Concentrations of individual PAH that are less than $0.5 \mathrm{ng} / \mathrm{m}^{3}$ (the reporting level) are estimated. $\mu \mathrm{g} / \mathrm{m}^{3}$, microgram per cubic meter; E, estimated concentration; GCMS, gas chromatography mass spectrometry; GFF, glass fiber filter; $\mathrm{m}^{3}$, cubic meter; PSE, pressurized solvent extraction; PUF, polyurethane foam; Scan, full scan ion monitoring mode; SIM, selected ion monitoring mode; PSE, pressurized solvent extraction; - , compound not detected at a concentration above the laboratory reporting level]

\begin{tabular}{|c|c|c|c|c|c|c|c|c|c|c|c|c|}
\hline $\begin{array}{c}\text { Begin } \\
\text { date } \\
\text { (mm/dd/yyyy) }\end{array}$ & Type & $\begin{array}{c}\text { Air } \\
\text { volume } \\
\text { sampled } \\
\left(\mathbf{m}^{3}\right)\end{array}$ & $\begin{array}{c}\text { Total } \\
\text { suspended } \\
\text { particulate } \\
\left(\mu \mathrm{g} / \mathrm{m}^{3}\right)\end{array}$ & $\begin{array}{c}\text { Sample } \\
\text { prepara- } \\
\text { tion set } \\
\text { number }\end{array}$ & $\begin{array}{c}\text { Extraction } \\
\text { by Soxhlet } \\
\text { or PSE }\end{array}$ & $\begin{array}{c}\text { Extract } \\
\text { split }\end{array}$ & $\begin{array}{l}\text { GCMS } \\
\text { SIM or } \\
\text { Scan }\end{array}$ & $\begin{array}{l}\text { C1-202 } \\
\text { Isomers, } \\
\text { methylated } \\
\text { fluoranthenes/ } \\
\text { pyrenes } \\
\text { (E) }\end{array}$ & $\begin{array}{c}\text { C1-228 } \\
\text { Isomers, } \\
\text { methylated } \\
\text { benzo[a] } \\
\text { anthracenes/ } \\
\text { chrysenes } \\
\text { (E) }\end{array}$ & $\begin{array}{c}\text { C1-252 } \\
\text { Isomers, } \\
\text { methylated } \\
\text { benzo- } \\
\text { / pyrenes/ } \\
\text { perylenes } \\
\text { (E) }\end{array}$ & $\begin{array}{l}\text { C2-178 } \\
\text { Isomers, } \\
\text { alkylated } \\
\text { phen- } \\
\text { anthrenes/ } \\
\text { anthracenes } \\
\text { (E) }\end{array}$ & $\begin{array}{c}\text { C2-202 } \\
\text { Isomers, } \\
\text { alkylated } \\
\text { fluor- } \\
\text { anthenes/ } \\
\text { s pyrenes } \\
\text { (E) }\end{array}$ \\
\hline \multirow[t]{4}{*}{$04 / 01 / 2003$} & GFF & 301.1 & 33.2 & 03.32308 & PSE & yes & SIM & - & - & - & - & - \\
\hline & Top PUF & & & 03.32308 & PSE & yes & SIM & 0.09 & - & - & 0.23 & - \\
\hline & $\begin{array}{c}\text { Bottom } \\
\text { PUF }\end{array}$ & & & 03.32308 & PSE & yes & Scan & - & - & - & - & - \\
\hline & TOTAL & & & & & & & 0.09 & - & - & 0.23 & - \\
\hline \multirow[t]{4}{*}{$04 / 29 / 2003$} & GFF & 315.3 & 40.0 & 03.32308 & PSE & yes & SIM & - & - & - & - & - \\
\hline & Top PUF & & & 03.32308 & PSE & yes & Scan & - & - & - & 0.47 & - \\
\hline & $\begin{array}{c}\text { Bottom } \\
\text { PUF }\end{array}$ & & & 03.32308 & PSE & yes & Scan & - & - & - & - & - \\
\hline & TOTAL & & & & & & & - & - & - & 0.47 & - \\
\hline \multirow[t]{4}{*}{$05 / 27 / 2003$} & GFF & 326.5 & 55.7 & 03.32308 & PSE & yes & SIM & - & - & - & - & - \\
\hline & Top PUF & & & 03.32308 & PSE & yes & Scan & - & - & - & 0.53 & - \\
\hline & $\begin{array}{c}\text { Bottom } \\
\text { PUF }\end{array}$ & & & 03.32308 & PSE & yes & Scan & - & - & - & - & - \\
\hline & TOTAL & & & & & & & - & - & - & 0.53 & - \\
\hline \multirow[t]{4}{*}{$07 / 22 / 2003$} & GFF & 307.8 & 52.6 & 03.32308 & PSE & yes & SIM & - & - & - & - & - \\
\hline & Top PUF & & & 03.32308 & PSE & yes & Scan & - & - & - & 0.94 & - \\
\hline & $\begin{array}{c}\text { Bottom } \\
\text { PUF }\end{array}$ & & & 03.32308 & PSE & yes & Scan & - & - & - & - & - \\
\hline & TOTAL & & & & & & & - & - & - & 0.94 & - \\
\hline \multirow[t]{4}{*}{$08 / 26 / 2003$} & GFF & 311.9 & 71.8 & 03.32308 & PSE & yes & SIM & - & - & - & - & - \\
\hline & Top PUF & & & 03.32308 & PSE & yes & Scan & - & - & - & 0.97 & - \\
\hline & $\begin{array}{c}\text { Bottom } \\
\text { PUF }\end{array}$ & & & 03.32308 & PSE & yes & Scan & - & - & - & - & - \\
\hline & TOTAL & & & & & & & - & - & - & 0.97 & - \\
\hline
\end{tabular}


Table 10B. Analytical results for polycyclic aromatic hydrocarbon (PAH) and alkylated PAH concentrations in air from the Sweetwater Reservoir atmospheric site, San Diego County, California.-Continued

[The site identification number is 324141117001601 . Values are given in nanograms per cubic meter $\left(\mathrm{ng} / \mathrm{m}^{3}\right)$ unless noted. Concentrations of individual PAH that are less than $0.5 \mathrm{ng} / \mathrm{m}^{3}$ (the reporting level) are estimated. $\mu \mathrm{g} / \mathrm{m}^{3}$, microgram per cubic meter; E, estimated concentration; GCMS, gas chromatography mass spectrometry; GFF, glass fiber filter; $\mathrm{m}^{3}$, cubic meter; PSE, pressurized solvent extraction; PUF, polyurethane foam; Scan, full scan ion monitoring mode; SIM, selected ion monitoring mode; PSE, pressurized solvent extraction; - , compound not detected at a concentration above the laboratory reporting level]

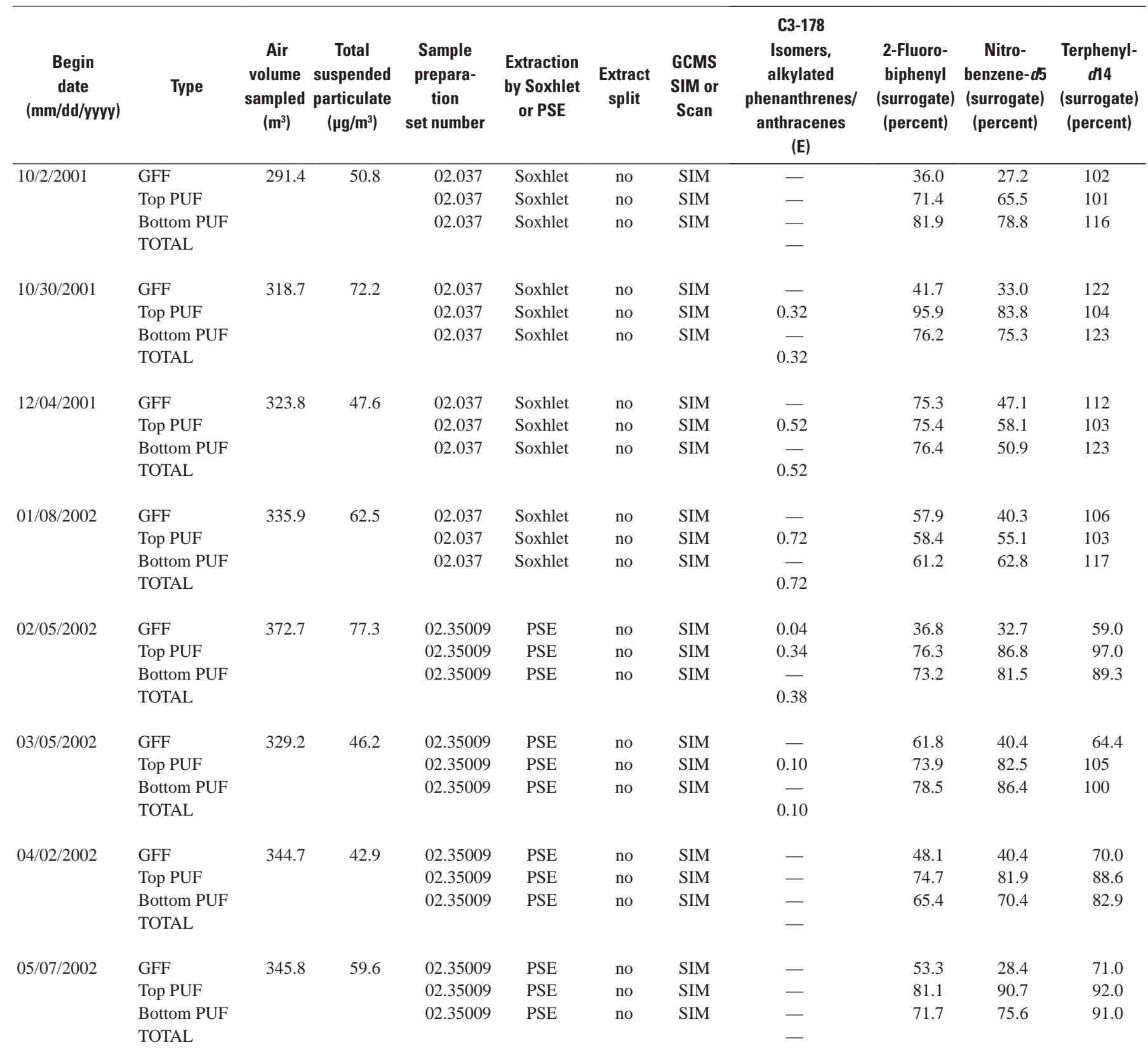


Table 10B. Analytical results for polycyclic aromatic hydrocarbon (PAH) and alkylated PAH concentrations in air from the Sweetwater Reservoir atmospheric site, San Diego County, California.-Continued

[The site identification number is 324141117001601 . Values are given in nanograms per cubic meter $\left(\mathrm{ng} / \mathrm{m}^{3}\right)$ unless noted. Concentrations of individual PAH that are less than $0.5 \mathrm{ng} / \mathrm{m}^{3}$ (the reporting level) are estimated. $\mu \mathrm{g} / \mathrm{m}^{3}$, microgram per cubic meter; E, estimated concentration; GCMS, gas chromatography mass spectrometry; GFF, glass fiber filter; $\mathrm{m}^{3}$, cubic meter; PSE, pressurized solvent extraction; PUF, polyurethane foam; Scan, full scan ion monitoring mode; SIM, selected ion monitoring mode; PSE, pressurized solvent extraction; - , compound not detected at a concentration above the laboratory reporting level]

\begin{tabular}{|c|c|c|c|c|c|c|c|c|c|c|c|}
\hline $\begin{array}{c}\text { Begin } \\
\text { date } \\
\text { (mm/dd/yyyy) }\end{array}$ & Type & $\begin{array}{c}\text { Air } \\
\text { volume } \\
\text { sampled } \\
\left(\mathbf{m}^{3}\right)\end{array}$ & $\begin{array}{c}\text { Total } \\
\text { suspended } \\
\text { particulate } \\
\left(\mu \mathrm{g} / \mathrm{m}^{3}\right)\end{array}$ & $\begin{array}{c}\text { Sample } \\
\text { prepara- } \\
\text { tion set } \\
\text { number }\end{array}$ & $\begin{array}{l}\text { Extraction } \\
\text { by Soxhlet } \\
\text { or PSE }\end{array}$ & $\begin{array}{c}\text { Extract } \\
\text { split }\end{array}$ & $\begin{array}{l}\text { GCMS } \\
\text { SIM or } \\
\text { Scan }\end{array}$ & $\begin{array}{l}\text { C3-178 Isomers, } \\
\text { alkylated } \\
\text { phenanthrenes/ } \\
\text { anthracenes } \\
\text { (E) }\end{array}$ & $\begin{array}{l}\text { 2-Fluoro- } \\
\text { biphenyl } \\
\text { (surrogate) } \\
\text { (percent) }\end{array}$ & $\begin{array}{c}\text { Nitro- } \\
\text { benzene-d5 } \\
\text { (surrogate) } \\
\text { (percent) }\end{array}$ & $\begin{array}{c}\text { Terphenyl- } \\
\quad \text { d44 } \\
\text { (surrogate) } \\
\text { (percent) }\end{array}$ \\
\hline \multirow[t]{4}{*}{ 06/04/2002 } & GFF & 317.3 & 54.8 & 02.35009 & PSE & no & SIM & - & 59.6 & 44.3 & 71.8 \\
\hline & Top PUF & & & 02.35009 & PSE & no & SIM & - & 63.9 & 62.6 & 83.7 \\
\hline & Bottom PUF & & & 02.35009 & PSE & no & SIM & - & 82.0 & 85.8 & 100 \\
\hline & TOTAL & & & & & & & - & & & \\
\hline \multirow[t]{4}{*}{ 07/02/2002 } & GFF & 328.5 & 59.1 & 02.35009 & PSE & no & SIM & - & 68.7 & 53.7 & 76.9 \\
\hline & Top PUF & & & 02.35009 & PSE & no & SIM & 0.16 & 70.2 & 71.4 & 82.0 \\
\hline & Bottom PUF & & & 02.35009 & PSE & no & SIM & - & 60.6 & 61.9 & 84.6 \\
\hline & TOTAL & & & & & & & 0.16 & & & \\
\hline \multirow[t]{4}{*}{ 08/06/2002 } & GFF & 262.4 & 71.6 & 02.35009 & PSE & no & SIM & - & 58.6 & 43.5 & 66.1 \\
\hline & Top PUF & & & 02.35009 & PSE & no & SIM & 0.27 & 69.0 & 72.9 & 91.3 \\
\hline & Bottom PUF & & & 02.35009 & PSE & no & SIM & - & 70.2 & 74.2 & 90.6 \\
\hline & TOTAL & & & & & & & 0.27 & & & \\
\hline \multirow[t]{4}{*}{ 10/01/2002 } & GFF & 315.9 & 62.0 & 02.35009 & PSE & no & SIM & - & 44.6 & 35.0 & 62.5 \\
\hline & Top PUF & & & 02.35009 & PSE & no & SIM & 0.22 & 65.6 & 64.9 & 91.9 \\
\hline & Bottom PUF & & & 02.35009 & PSE & no & SIM & - & 59.4 & 59.1 & 87.3 \\
\hline & TOTAL & & & & & & & 0.22 & & & \\
\hline \multirow[t]{4}{*}{$11 / 26 / 2002$} & GFF & 301.3 & 47.8 & 02.35009 & PSE & no & SIM & - & 50.8 & 40.1 & 72.0 \\
\hline & Top PUF & & & 02.35009 & PSE & no & SIM & 0.33 & 67.0 & 67.6 & 88.2 \\
\hline & Bottom PUF & & & 02.35009 & PSE & no & SIM & - & 69.8 & 74.7 & 90.1 \\
\hline & TOTAL & & & & & & & 0.33 & & & \\
\hline \multirow[t]{4}{*}{ 01/07/2003 } & GFF & 294.8 & 53.6 & 03.32308 & PSE & yes & SIM & - & 76.5 & 76.5 & 88.2 \\
\hline & Top PUF & & & 03.32308 & PSE & yes & Scan & 0.69 & 94.1 & 129 & 106 \\
\hline & Bottom PUF & & & 03.32308 & PSE & yes & Scan & - & 100 & 124 & 112 \\
\hline & TOTAL & & & & & & & 0.69 & & & \\
\hline \multirow[t]{4}{*}{ 02/04/2003 } & GFF & 312.1 & 36.5 & 03.32308 & PSE & yes & SIM & - & 75.0 & 75.0 & 93.8 \\
\hline & Top PUF & & & 03.32308 & PSE & yes & Scan & - & 113 & 125 & 119 \\
\hline & Bottom PUF & & & 03.32308 & PSE & yes & Scan & - & 100 & 131 & 113 \\
\hline & TOTAL & & & & & & & - & & & \\
\hline \multirow[t]{4}{*}{ 03/04/2003 } & GFF & 283.0 & 53.0 & 03.32308 & PSE & yes & Scan & - & 82.2 & 102 & 102 \\
\hline & Top PUF & & & 03.32308 & PSE & yes & Scan & - & 88.9 & 122 & 100 \\
\hline & Bottom PUF & & & 03.32308 & PSE & yes & Scan & - & 94.4 & 106 & 106 \\
\hline & TOTAL & & & & & & & - & & & \\
\hline
\end{tabular}


Table 10B. Analytical results for polycyclic aromatic hydrocarbon (PAH) and alkylated PAH concentrations in air from the Sweetwater Reservoir atmospheric site, San Diego County, California.-Continued

[The site identification number is 324141117001601 . Values are given in nanograms per cubic meter $\left(\mathrm{ng} / \mathrm{m}^{3}\right)$ unless noted. Concentrations of individual PAHs that are less than $0.5 \mathrm{ng} / \mathrm{m}^{3}$ (the reporting level) are estimated. $\mu \mathrm{g} / \mathrm{m}^{3}$, microgram per cubic meter; E, estimated concentration; GCMS, gas chromatography mass spectrometry; GFF, glass fiber filter; $\mathrm{m}^{3}$, cubic meter; PSE, pressurized solvent extraction; PUF, polyurethane foam; Scan, full scan ion monitoring mode; SIM, selected ion monitoring mode; PSE, pressurized solvent extraction; - , compound not detected at a concentration above the laboratory reporting level]

\begin{tabular}{|c|c|c|c|c|c|c|c|c|c|c|c|}
\hline $\begin{array}{c}\text { Begin } \\
\text { date } \\
\text { (mm/dd/yyyy) }\end{array}$ & Type & $\begin{array}{c}\text { Air } \\
\text { volume } \\
\text { sampled } \\
\left(\mathbf{m}^{3}\right)\end{array}$ & $\begin{array}{c}\text { Total } \\
\text { suspended } \\
\text { particulate } \\
\left(\mu \mathrm{g} / \mathrm{m}^{3}\right)\end{array}$ & $\begin{array}{c}\text { Sample } \\
\text { prepara- } \\
\text { tion set } \\
\text { number }\end{array}$ & $\begin{array}{c}\text { Extraction } \\
\text { by Soxhlet } \\
\text { or PSE }\end{array}$ & $\begin{array}{c}\text { Extract } \\
\text { split }\end{array}$ & $\begin{array}{l}\text { GCMS } \\
\text { SIM or } \\
\text { Scan }\end{array}$ & $\begin{array}{l}\text { C3-178 Isomers, } \\
\text { alkylated } \\
\text { phenanthrenes/ } \\
\text { anthracenes } \\
\text { (E) }\end{array}$ & $\begin{array}{l}\text { 2-Fluoro- } \\
\text { biphenyl, } \\
\text { surrogate } \\
\text { (percent) }\end{array}$ & $\begin{array}{c}\text { Nitro- } \\
\text { benzene- } 45, \\
\text { surrogate } \\
\text { (percent) }\end{array}$ & $\begin{array}{c}\text { Terphenyl- } \\
\text { d14, } \\
\text { surrogate } \\
\text { (percent) }\end{array}$ \\
\hline \multirow[t]{4}{*}{$04 / 01 / 2003$} & GFF & 301.1 & 33.2 & 03.32308 & PSE & yes & SIM & - & 76.5 & 76.5 & 94.1 \\
\hline & Top PUF & & & 03.32308 & PSE & yes & SIM & - & 70.6 & 88.2 & 88.0 \\
\hline & Bottom PUF & & & 03.32308 & PSE & yes & Scan & - & 94.1 & 118 & 100 \\
\hline & TOTAL & & & & & & & - & & & \\
\hline \multirow[t]{4}{*}{$04 / 29 / 2003$} & GFF & 315.3 & 40.0 & 03.32308 & PSE & yes & SIM & - & 75.0 & 68.8 & 87.5 \\
\hline & Top PUF & & & 03.32308 & PSE & yes & Scan & - & 100 & 125 & 113 \\
\hline & Bottom PUF & & & 03.32308 & PSE & yes & Scan & - & 93.8 & 87.5 & 106 \\
\hline & TOTAL & & & & & & & - & & & \\
\hline \multirow[t]{4}{*}{$05 / 27 / 2003$} & GFF & 326.5 & 55.7 & 03.32308 & PSE & yes & SIM & - & 73.3 & 66.7 & 93.3 \\
\hline & Top PUF & & & 03.32308 & PSE & yes & Scan & - & 107 & 153 & 113 \\
\hline & Bottom PUF & & & 03.32308 & PSE & yes & Scan & - & 107 & 127 & 107 \\
\hline & TOTAL & & & & & & & - & & & \\
\hline \multirow[t]{4}{*}{$07 / 22 / 2003$} & GFF & 307.8 & 52.6 & 03.32308 & PSE & yes & SIM & - & 68.8 & 58.1 & 93.8 \\
\hline & Top PUF & & & 03.32308 & PSE & yes & Scan & - & 106 & 125 & 106 \\
\hline & Bottom PUF & & & 03.32308 & PSE & yes & Scan & - & 100 & 113 & 100 \\
\hline & TOTAL & & & & & & & - & & & \\
\hline \multirow[t]{4}{*}{$08 / 26 / 2003$} & GFF & 311.9 & 71.8 & 03.32308 & PSE & yes & SIM & - & 61.9 & 48.1 & 87.5 \\
\hline & Top PUF & & & 03.32308 & PSE & yes & Scan & - & 93.8 & 125 & 100 \\
\hline & Bottom PUF & & & 03.32308 & PSE & yes & Scan & - & 106 & 156 & 100 \\
\hline & TOTAL & & & & & & & - & & & \\
\hline
\end{tabular}


Table 11A. Pesticide compounds analyzed using method Airpest001 for air samples collected from the Sweetwater Reservoir atmospheric site, San Diego County, California.

[The site identification number is 324141117001601 . Estimated reporting level assumes 315 cubic meters as the average air volume. $\mathrm{ng} / \mathrm{m}^{3}$, nanogram per cubic meter]

\begin{tabular}{|c|c|}
\hline Compound & $\begin{array}{c}\text { Estimated } \\
\text { reporting level } \\
\left(\mathrm{ng} / \mathrm{m}^{3}\right)\end{array}$ \\
\hline Acetochlor & 0.03 \\
\hline Alachlor & 0.03 \\
\hline Atrazine & 0.03 \\
\hline Azinphos-methyl $^{1}$ & 0.02 \\
\hline Benfluralin $^{1}$ & 0.30 \\
\hline Carbaryl $^{1}$ & 0.05 \\
\hline Carbofuran & 0.05 \\
\hline Chlorpyrifos & 0.06 \\
\hline Cyanazine & 0.06 \\
\hline Dacthal (DCPA) & 0.03 \\
\hline$p, p^{\prime}-\mathrm{DDE}$ & 0.10 \\
\hline Deethylatrazine & 0.05 \\
\hline Diazinon & 0.03 \\
\hline Dieldrin & 0.02 \\
\hline Disulfoton $^{1}$ & 0.27 \\
\hline Ethalfluralin $^{1}$ & 0.30 \\
\hline Ethion & 0.03 \\
\hline Ethoprophos & 0.05 \\
\hline Fonofos & 0.05 \\
\hline$\alpha-\mathrm{HCH}$ & 0.03 \\
\hline Lindane & 0.06 \\
\hline Linuron $^{1}$ & 0.03 \\
\hline Malathion & 0.08 \\
\hline Metolachlor & 0.03 \\
\hline Metribuzin & 0.06 \\
\hline Molinate $^{1}$ & 0.50 \\
\hline Napropamide & 0.05 \\
\hline Parathion & 0.06 \\
\hline Parathion-methyl & 0.10 \\
\hline Pendimethalin & 0.06 \\
\hline cis-Permethrin & 0.08 \\
\hline Phorate & 0.03 \\
\hline Prometon $^{1}$ & 0.29 \\
\hline Propyzamide & 0.05 \\
\hline Propachlor & 0.11 \\
\hline Propanil & 0.06 \\
\hline Propargite I \& II, & 0.21 \\
\hline Simazine & 0.08 \\
\hline Tebuthiuron $^{1}$ & 0.45 \\
\hline Terbufos ${ }^{1}$ & 0.21 \\
\hline Thiobencarb & 0.03 \\
\hline Tri-allate & 0.02 \\
\hline Trifluralin $^{1}$ & 0.03 \\
\hline Diazinon- $d 10$ (surrogate) & percent \\
\hline$\alpha-\mathrm{HCH}-d 6$ (surrogate) & percent \\
\hline
\end{tabular}

${ }^{1}$ Concentration estimated. 


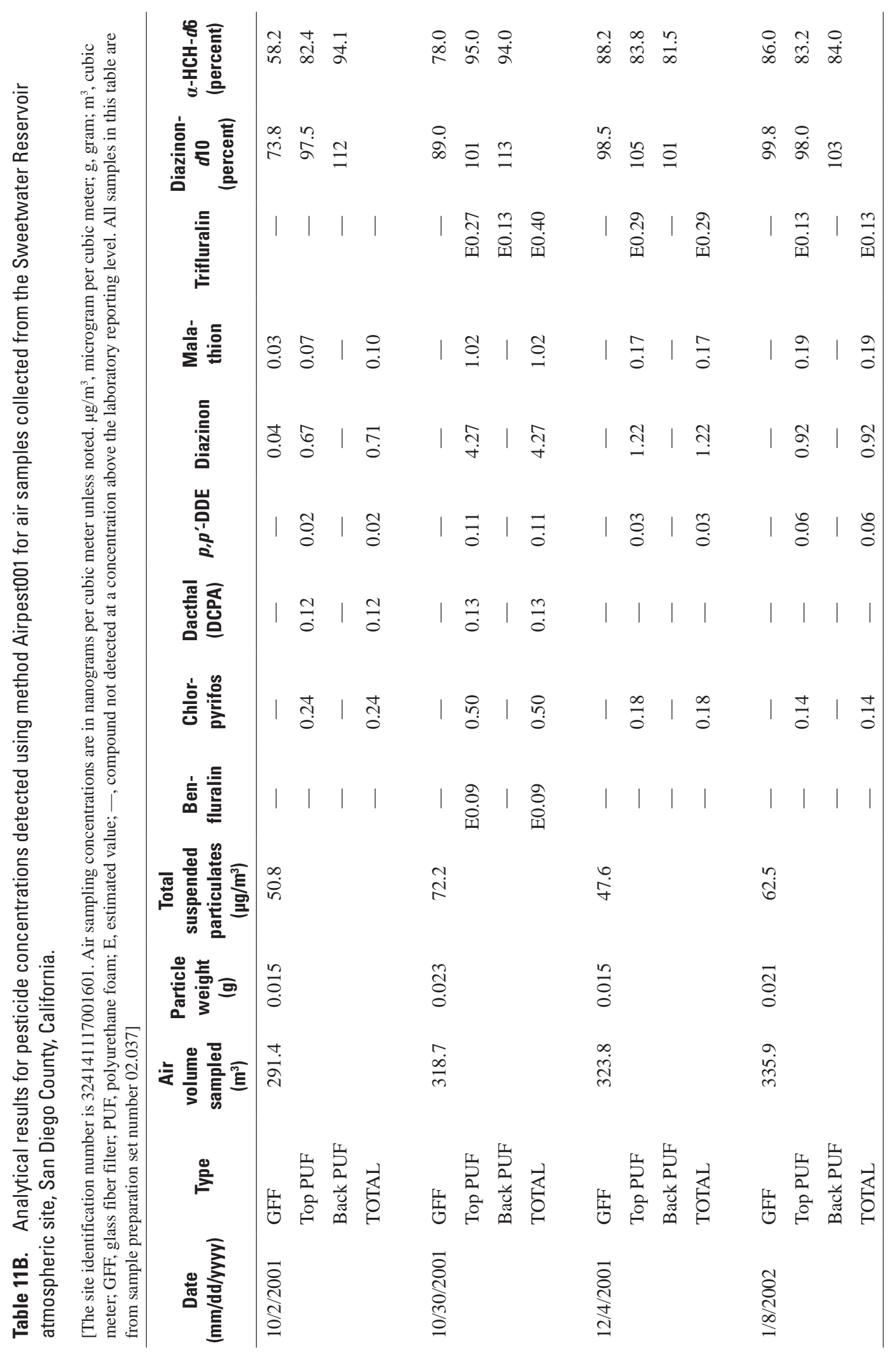


Table 12A. Pesticide compounds analyzed using method Airpest002 for air samples collected from the Sweetwater Reservoir atmospheric site, California.

[The site identification number is 324141117001601 . Estimated reporting level assumes 315 cubic meters as the average air volume. ng/ $\mathrm{m}^{3}$, nanogram per cubic meter]

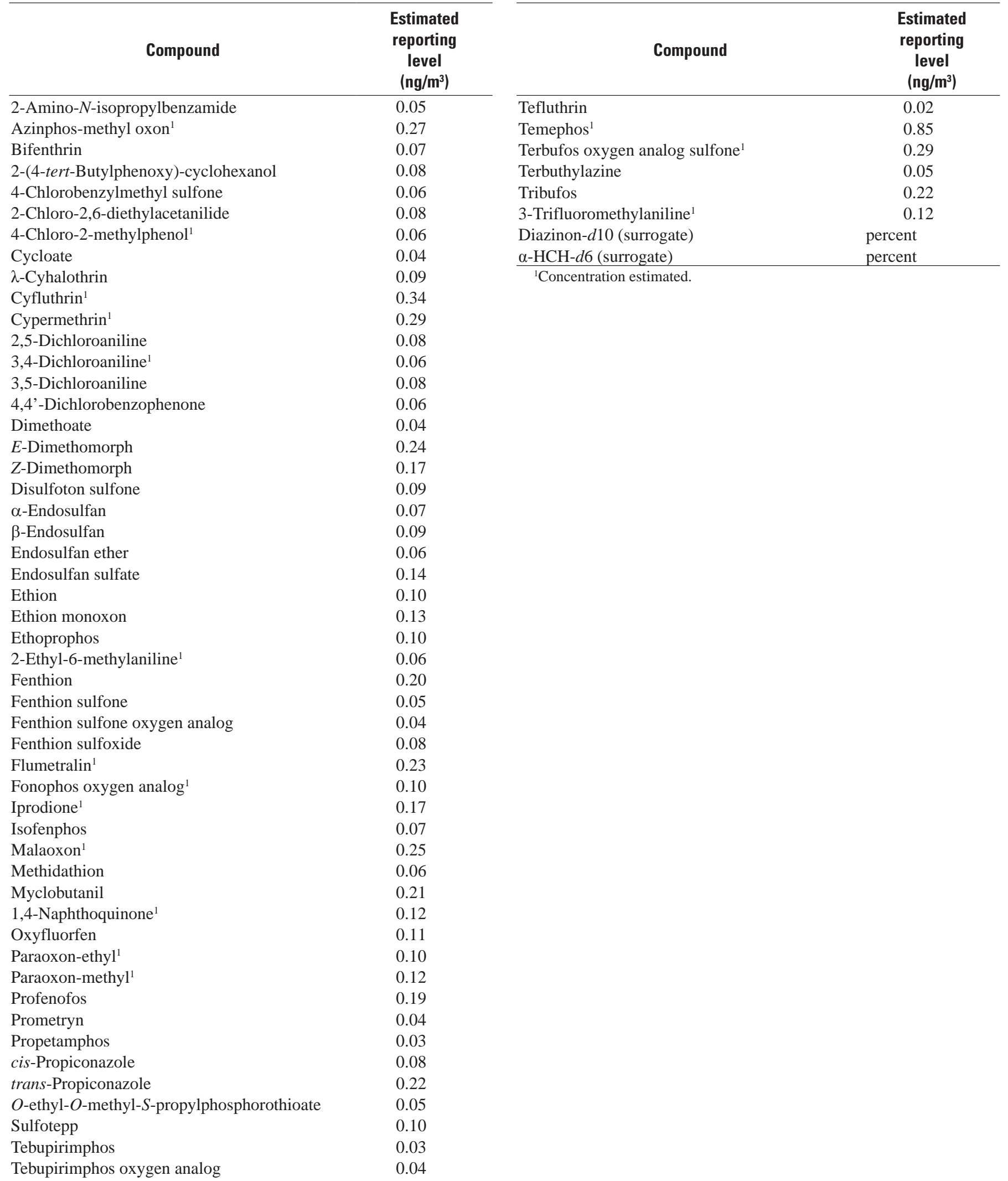


Table 12B. Analytical results for pesticide concentrations detected using method Airpest002 for air samples collected from the Sweetwater Reservoir atmospheric site, San diego County, California.

[The site identification number is 324141117001601. All samples in this table are from sample preparation set number 02.037. Air sampling concentrations are in nanograms per cubic meter unless noted. $\mu \mathrm{g} / \mathrm{m}^{3}$, microgram per cubic meter; g, gram; $\mathrm{m}^{3}$, cubic meter; GFF, glass fiber filter; PUF, polyurethane foam; E, estimated value; - , compound not detected at a concentration above the laboratory reporting level]

\begin{tabular}{|c|c|c|c|c|c|c|c|c|c|}
\hline $\begin{array}{c}\text { Date } \\
\text { (mm/dd/yyyy) }\end{array}$ & Type & $\begin{array}{c}\text { Air volume } \\
\text { sampled } \\
\left(\mathrm{m}^{3}\right)\end{array}$ & $\begin{array}{c}\text { Particle } \\
\text { weight } \\
\text { (g) }\end{array}$ & $\begin{array}{c}\text { Total } \\
\text { suspended } \\
\text { particulate } \\
\left(\mathbf{m g} / \mathbf{m}^{3}\right)\end{array}$ & Bifenthrin & Malaoxon & $\begin{array}{c}\text { 1,4- } \\
\text { Naphtho- } \\
\text { quinone }\end{array}$ & $\begin{array}{c}\text { Diazinon- } \\
\text { d10 } \\
\text { (percent) }\end{array}$ & $\begin{array}{c}\alpha-\mathrm{HCH}-d 6 \\
\text { (percent) }\end{array}$ \\
\hline \multirow[t]{4}{*}{$10 / 02 / 2001$} & GFF & 291.4 & 0.015 & 50.8 & E0.05 & E0.30 & - & 79.7 & 57.1 \\
\hline & Top PUF & & & & - & - & - & 94.0 & 78.0 \\
\hline & Bottom PUF & & & & - & - & - & 92.2 & 84.4 \\
\hline & TOTAL & & & & E0.05 & E0.30 & - & & \\
\hline \multirow[t]{4}{*}{$10 / 30 / 2001$} & GFF & 318.7 & 0.023 & 72.2 & E0.05 & E0.24 & - & 100 & 76.8 \\
\hline & Top PUF & & & & - & - & - & 96.6 & 81.9 \\
\hline & Bottom PUF & & & & - & - & - & 81.7 & 75.4 \\
\hline & TOTAL & & & & E0.05 & E0.24 & - & & \\
\hline \multirow[t]{4}{*}{$12 / 04 / 2001$} & GFF & 323.8 & 0.015 & 47.6 & E0.06 & E0.09 & - & 99.9 & 78.0 \\
\hline & Top PUF & & & & - & - & E0.22 & 90.0 & 83.9 \\
\hline & Bottom PUF & & & & 一 & - & E0.07 & 91.1 & 78.8 \\
\hline & TOTAL & & & & E0.06 & E0.09 & E0.29 & & \\
\hline \multirow[t]{4}{*}{$01 / 08 / 2002$} & GFF & 335.9 & 0.021 & 62.5 & - & - & - & 94.4 & 79.3 \\
\hline & Top PUF & & & & - & - & - & 84.6 & 84.1 \\
\hline & Bottom PUF & & & & - & - & - & 78.4 & 67.0 \\
\hline & TOTAL & & & & - & - & - & & \\
\hline
\end{tabular}


Table 13A. Pesticide compounds analyzed using method Airpest003 for air samples collected from the Sweetwater Reservoir atmospheric site, San Diego County, California.

[The site identification number is 324141117001601 . Estimated reporting level assumes 315 cubic meters as the average air volume. ng/m³ , nanogram per cubic meter]

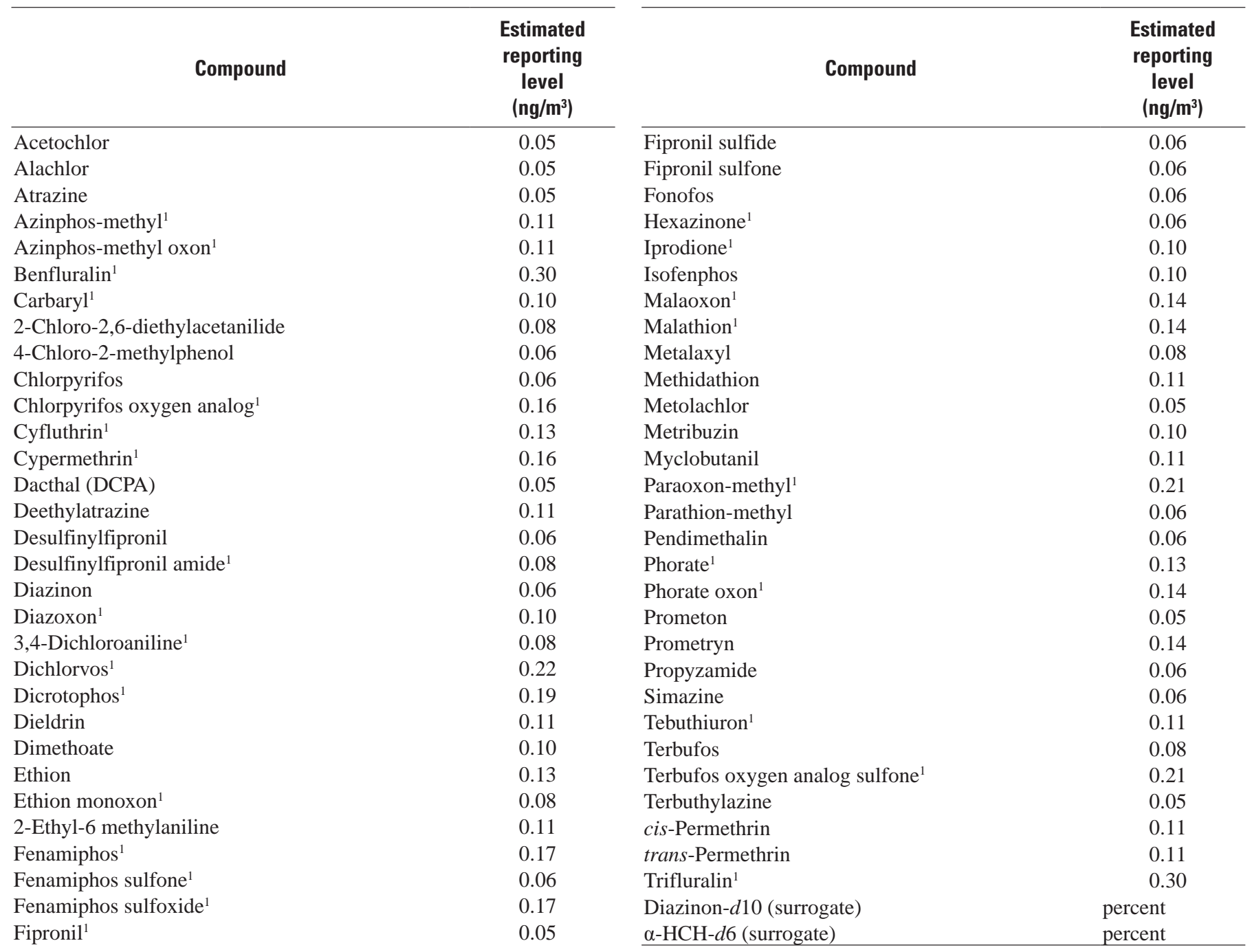

${ }^{1}$ Concentration estimated. 
Table 13B. Analytical results for pesticide concentrations detected using method Airpest003 for air samples collected from the Sweetwater Reservoir atmospheric site, San Diego County, California.

[The site identification number is 324141117001601 . Air sampling concentrations are in nanograms per cubic meter unless noted. $\mu \mathrm{g} / \mathrm{m}^{3}, \mathrm{microgram}$ per cubic meter; g, gram; $\mathrm{m}^{3}$, cubic meter; GFF, glass fiber filter; PUF, polyurethane foam; E, estimated value; —, compound not detected at a concentration above the laboratory reporting level]

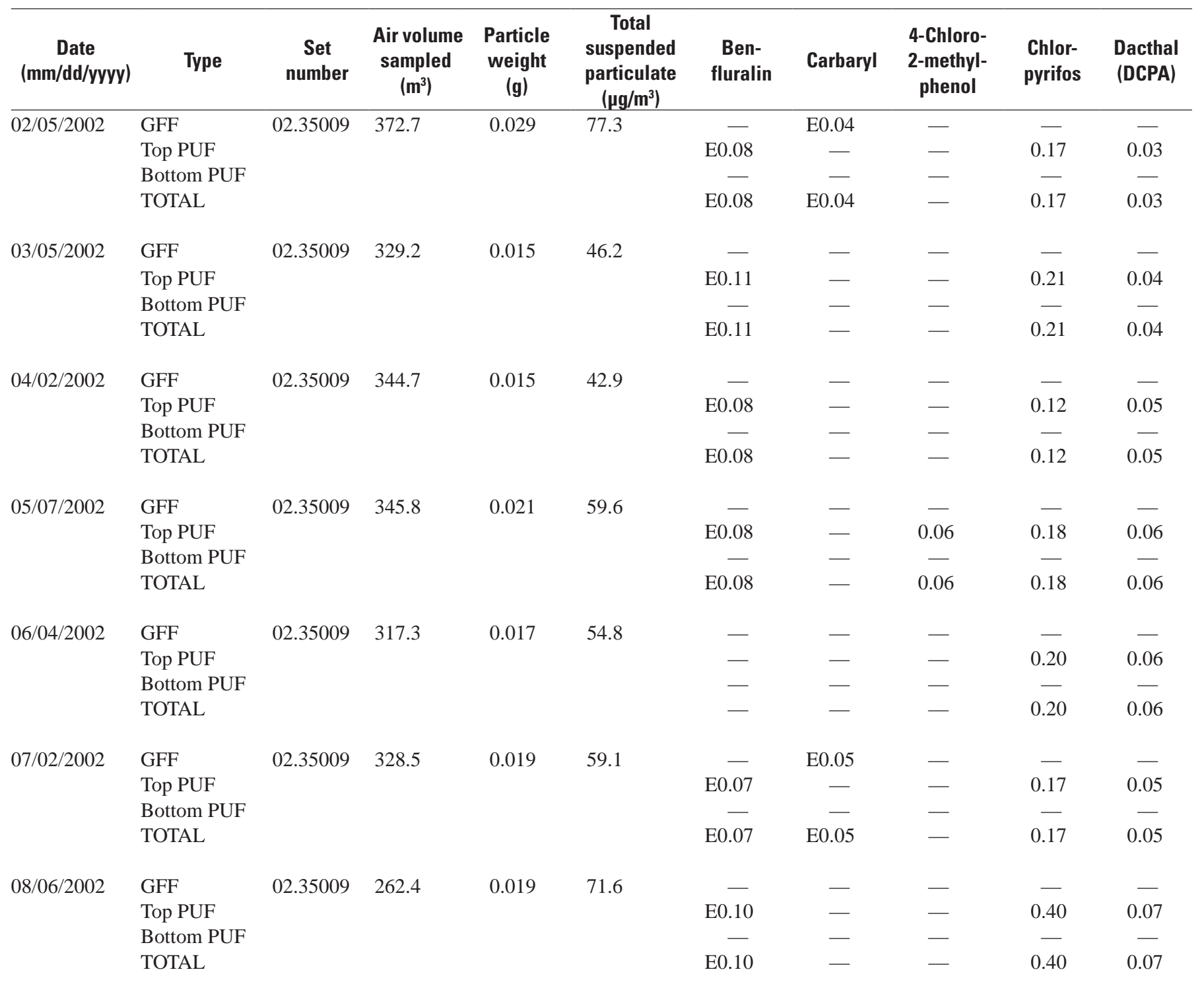


Table 13B. Analytical results for pesticide concentrations detected using method Airpest003 for air samples collected from the Sweetwater Reservoir atmospheric site, San Diego County, California.-Continued

[The site identification number is 324141117001601 . Air sampling concentrations in nanograms per cubic meter unless noted. $\mu \mathrm{g} / \mathrm{m}^{3}, \mathrm{microgram}$ per cubic meter; $\mathrm{g}$, gram; $\mathrm{m}^{3}$, cubic meter; GFF, glass fiber filter; PUF, polyurethane foam; E, estimated value; —, compound not detected at a concentration above the laboratory reporting level]

\begin{tabular}{|c|c|c|c|c|c|c|c|c|c|c|c|}
\hline $\begin{array}{l}\text { Deethyl- } \\
\text { atrazine }\end{array}$ & Diazinon & Diazoxon & $\begin{array}{l}\text { Ipro- } \\
\text { dione }\end{array}$ & $\begin{array}{l}\text { Mala- } \\
\text { oxon }\end{array}$ & Malathion & Simazine & $\begin{array}{c}\text { cis- } \\
\text { Per- } \\
\text { methrin }\end{array}$ & $\begin{array}{c}\text { trans- } \\
\text { Per- } \\
\text { methrin }\end{array}$ & $\begin{array}{c}\text { Tri- } \\
\text { fluralin }\end{array}$ & $\begin{array}{c}\text { Diazinon-d10 } \\
\text { (surrogate) } \\
\text { (percent) }\end{array}$ & $\begin{array}{c}\alpha-\mathrm{HCH}-\mathrm{d} 6 \\
\text { (surrogate) } \\
\text { (percent) }\end{array}$ \\
\hline- & - & - & - & - & - & 0.02 & - & - & - & 59.6 & 44.7 \\
\hline- & 0.65 & E0.08 & - & - & E0.12 & - & - & - & E0.21 & 85.7 & 67.1 \\
\hline- & - & - & - & - & - & - & - & - & E0.07 & 78.3 & 59.6 \\
\hline- & 0.65 & E0.08 & - & - & E0.12 & 0.02 & - & - & Е0.29 & & \\
\hline- & - & - & - & E0.09 & - & - & - & - & - & 79.0 & 59.3 \\
\hline- & 0.99 & E0.07 & - & - & E0.16 & - & - & - & E0.60 & 92.2 & 62.5 \\
\hline- & - & - & - & - & - & - & - & - & E0.07 & 79.0 & 62.5 \\
\hline- & 0.99 & E0.07 & - & E0.09 & E0.16 & - & - & - & E0.67 & & \\
\hline- & 0.03 & - & - & E0.10 & - & - & - & - & - & 65.5 & 48.3 \\
\hline- & 0.17 & - & - & - & - & - & - & - & E0.18 & 75.8 & 58.6 \\
\hline- & - & - & - & - & - & - & - & - & E0.06 & 72.4 & 55.2 \\
\hline- & 0.20 & - & - & E0.10 & - & - & - & - & E0.24 & & \\
\hline- & 0.04 & - & - & E0.14 & E0.08 & - & 0.07 & 0.07 & - & 69.2 & 45.0 \\
\hline- & 0.63 & E0.06 & - & - & E0.14 & - & - & - & E0.28 & 79.5 & 62.2 \\
\hline- & - & - & - & - & - & - & - & - & E0.08 & 79.5 & 62.2 \\
\hline- & 0.68 & E0.06 & - & E0.14 & E0.22 & - & 0.07 & 0.07 & E0.36 & & \\
\hline- & - & - & - & E0.16 & - & - & 0.07 & 0.07 & - & 69.8 & 50.8 \\
\hline- & 0.27 & - & - & - & E0.09 & - & - & - & E0.15 & 82.5 & 60.3 \\
\hline- & - & - & - & - & - & - & - & - & E0.11 & 83.3 & 65.5 \\
\hline- & 0.27 & - & - & E0.16 & E0.09 & - & 0.07 & 0.07 & E0.26 & & \\
\hline- & - & - & - & E0.19 & E0.06 & - & 0.07 & 0.08 & - & 75.6 & 55.8 \\
\hline- & 0.55 & - & - & - & E0.12 & - & - & - & E0.15 & 74.5 & 55.0 \\
\hline- & - & - & - & - & - & - & - & - & E0.08 & 82.1 & 59.1 \\
\hline- & 0.55 & - & - & E0.19 & E0.18 & - & 0.07 & 0.08 & E0.23 & & \\
\hline 0.07 & - & - & - & E0.14 & - & - & 0.10 & 0.11 & - & 65.6 & 47.2 \\
\hline- & 1.73 & E0.12 & - & E0.42 & E0.42 & - & - & - & E0.36 & 86.6 & 60.4 \\
\hline- & - & - & - & - & - & - & - & - & E0.19 & 76.1 & 60.4 \\
\hline 0.07 & 1.73 & E0.12 & - & E0.56 & E0.42 & - & 0.10 & 0.11 & E0.55 & & \\
\hline
\end{tabular}


Table 13B. Analytical results for pesticide concentrations detected using method Airpest003 for air samples collected from the Sweetwater Reservoir atmospheric site, San Diego County, California.-Continued

[The site identification number is 324141117001601 . Air sampling concentrations are in nanograms per cubic meter unless noted. $\mu \mathrm{g} / \mathrm{m}^{3}, \mathrm{microgram}$ per cubic meter; g, gram; $\mathrm{m}^{3}$, cubic meter; GFF, glass fiber filter; PUF, polyurethane foam; E, estimated value; - , compound not detected at a concentration above the laboratory reporting level]

\begin{tabular}{|c|c|c|c|c|c|c|c|c|c|c|}
\hline $\begin{array}{c}\text { Date } \\
\text { (mm/dd/yyyv) }\end{array}$ & Type & $\begin{array}{c}\text { Set } \\
\text { number }\end{array}$ & $\begin{array}{l}\text { Air volume } \\
\text { sampled } \\
\left(\mathrm{m}^{3}\right)\end{array}$ & $\begin{array}{l}\text { Particle } \\
\text { weight } \\
\text { (g) }\end{array}$ & $\begin{array}{c}\text { Total } \\
\text { suspended } \\
\text { particulate } \\
\left(\mu \mathrm{g} / \mathrm{m}^{3}\right)\end{array}$ & $\begin{array}{l}\text { Ben- } \\
\text { fluralin }\end{array}$ & Carbaryl & $\begin{array}{l}\text { 4-Chloro- } \\
\text { 2-methyl- } \\
\text { phenol }\end{array}$ & $\begin{array}{l}\text { Chlor- } \\
\text { pyrifos }\end{array}$ & $\begin{array}{l}\text { Dacthal } \\
\text { (DCPA) }\end{array}$ \\
\hline \multirow[t]{5}{*}{$10 / 1 / 2002$} & GFF & 02.35009 & 315.9 & 0.020 & 62.0 & - & - & - & - & - \\
\hline & Top PUF & & & & & E0.09 & - & - & 0.33 & 0.06 \\
\hline & Bottom PUF & & & & & - & - & - & - & - \\
\hline & TOTAL & & & & & & - & - & 0.33 & 0.06 \\
\hline & & & & & & E0.09 & & & & \\
\hline \multirow[t]{4}{*}{$11 / 26 / 2002$} & GFF & 02.35009 & 301.3 & 0.014 & 47.8 & - & - & - & - & - \\
\hline & Top PUF & & & & & E0.10 & - & - & 0.19 & 0.07 \\
\hline & Bottom PUF & & & & & - & - & - & - & - \\
\hline & TOTAL & & & & & E0.10 & - & - & 0.19 & 0.07 \\
\hline \multirow[t]{4}{*}{$1 / 7 / 2003$} & GFF & 03.32308 & 294.8 & 0.016 & 53.6 & - & - & - & - & - \\
\hline & Top PUF & & & & & E0.07 & - & - & 0.15 & 0.05 \\
\hline & Bottom PUF & & & & & - & - & - & - & - \\
\hline & TOTAL & & & & & E0.07 & - & - & 0.15 & 0.05 \\
\hline \multirow[t]{4}{*}{$2 / 4 / 2003$} & GFF & 03.32308 & 312.1 & 0.011 & 36.5 & - & - & - & - & - \\
\hline & Top PUF & & & & & E0.03 & - & - & 0.06 & 0.03 \\
\hline & Bottom PUF & & & & & - & - & - & - & - \\
\hline & TOTAL & & & & & E0.03 & - & - & 0.06 & 0.03 \\
\hline \multirow[t]{4}{*}{$3 / 4 / 2003$} & GFF & 03.32308 & 283.0 & 0.015 & 53.0 & - & - & - & - & - \\
\hline & Top PUF & & & & & E0.07 & - & - & 0.12 & 0.06 \\
\hline & Bottom PUF & & & & & E0.03 & - & - & - & - \\
\hline & TOTAL & & & & & E0.10 & - & - & 0.12 & 0.06 \\
\hline \multirow[t]{4}{*}{ 4/1/2003 } & GFF & 03.32308 & 301.1 & 0.01 & 33.2 & - & - & - & - & - \\
\hline & Top PUF & & & & & E0.03 & - & - & 0.04 & 0.03 \\
\hline & Bottom PUF & & & & & - & - & - & - & - \\
\hline & TOTAL & & & & & E0.03 & - & - & 0.04 & 0.03 \\
\hline \multirow[t]{4}{*}{ 4/29/2003 } & GFF & 03.32308 & 315.3 & 0.013 & 40.0 & - & - & - & - & - \\
\hline & Top PUF & & & & & E0.03 & - & - & 0.06 & 0.05 \\
\hline & Bottom PUF & & & & & - & - & - & - & - \\
\hline & TOTAL & & & & & E0.03 & - & - & 0.06 & 0.05 \\
\hline
\end{tabular}


Table 13B. Analytical results for pesticide concentrations detected using method Airpest003 for air samples collected from the Sweetwater Reservoir atmospheric site, San Diego County, California.-Continued

[The site identification number is 324141117001601 . Air sampling concentrations in nanograms per cubic meter unless noted. $\mu \mathrm{g} / \mathrm{m}^{3}$, microgram per cubic meter; $\mathrm{g}$, gram; $\mathrm{m}^{3}$, cubic meter; GFF, glass fiber filter; PUF, polyurethane foam; E, estimated value; - , compound not detected at a concentration above the laboratory reporting level]

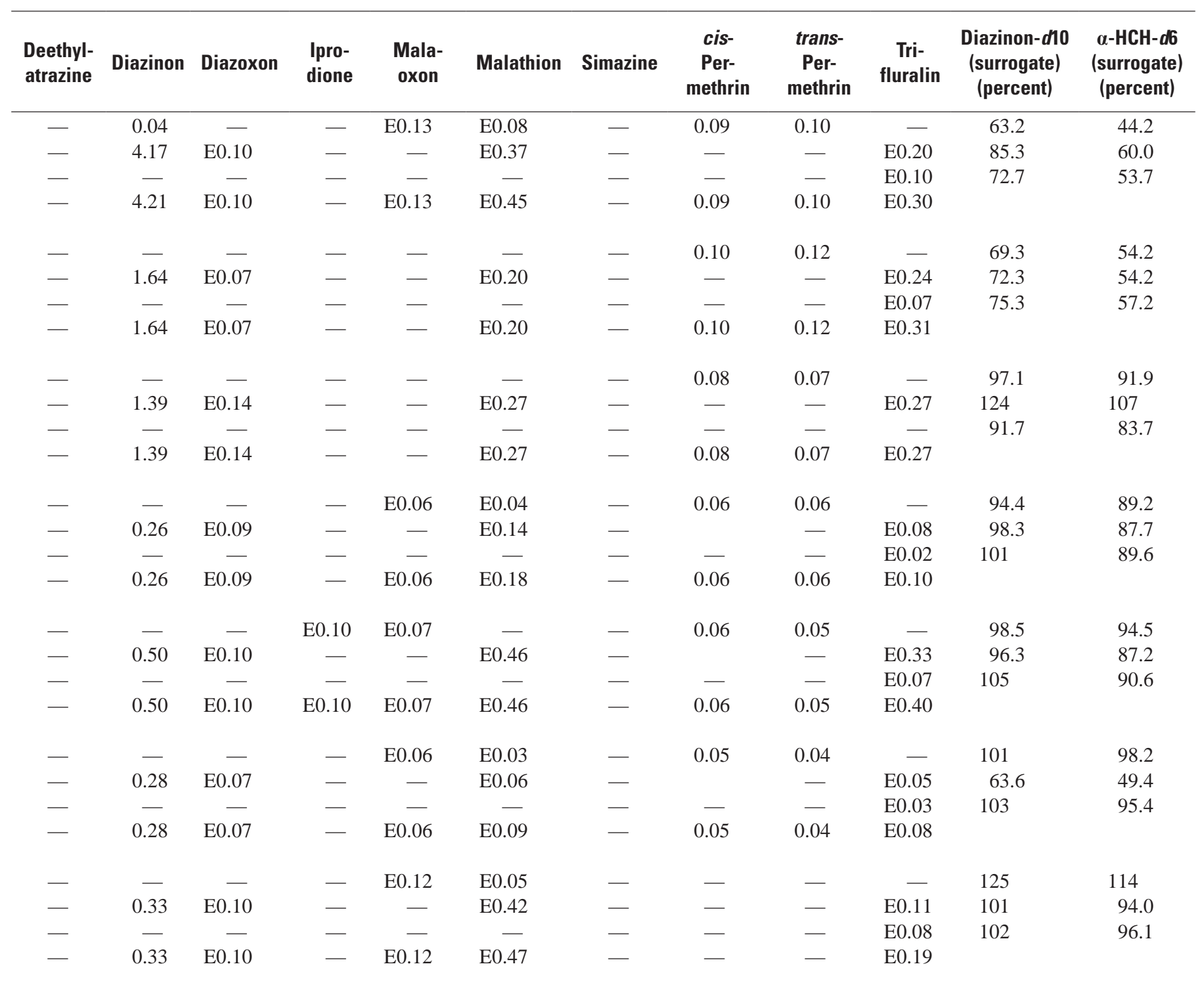


Table 13B. Analytical results for pesticide concentrations detected using method Airpest003 for air samples collected from the Sweetwater Reservoir atmospheric site, San Diego County, California.-Continued

[The site identification number is 324141117001601 . Air sampling concentrations are in nanograms per cubic meter unless noted. $\mu \mathrm{g} / \mathrm{m}^{3}$, microgram per cubic meter; g, gram; $\mathrm{m}^{3}$, cubic meter; GFF, glass fiber filter; PUF, polyurethane foam; E, estimated value; - , compound not detected at a concentration above the laboratory reporting level]

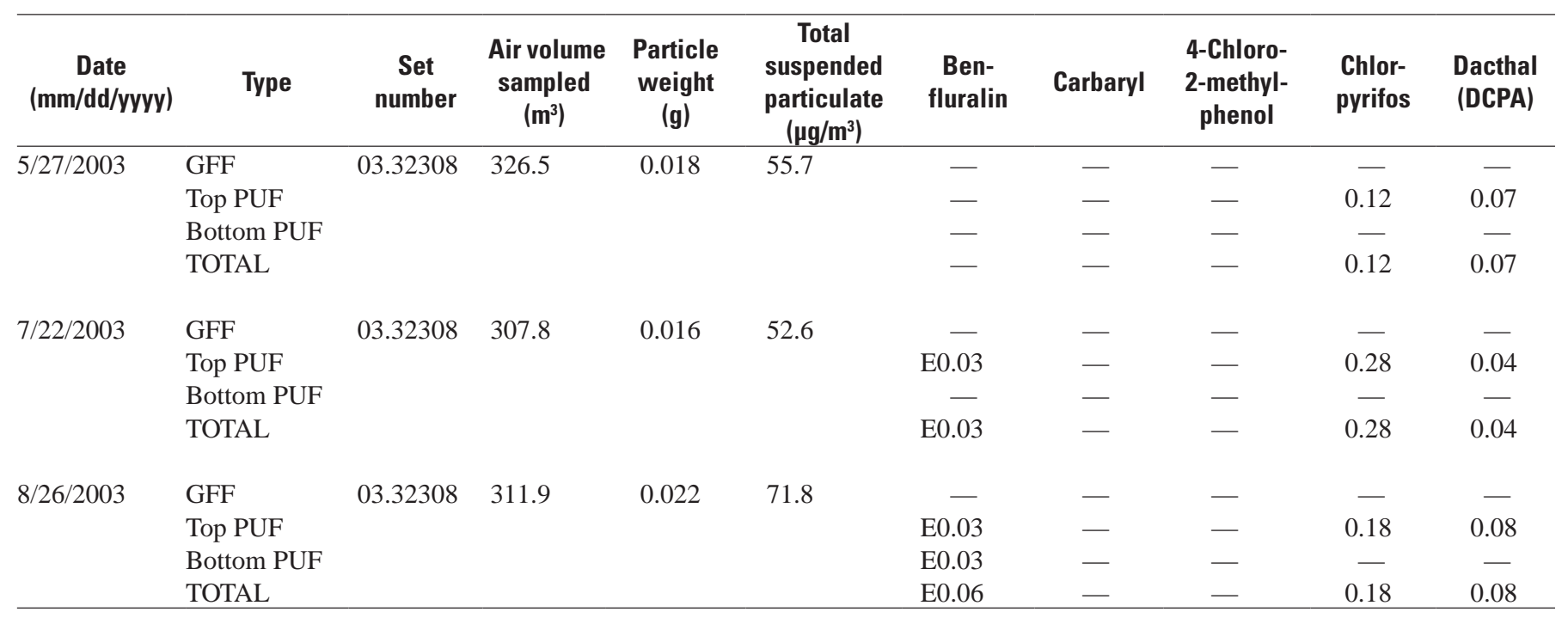


Table 13B. Analytical results for pesticide concentrations detected using method Airpest003 for air samples collected from the Sweetwater Reservoir atmospheric site, San Diego County, California.-Continued

[The site identification number is 324141117001601 . Air sampling concentrations in nanograms per cubic meter unless noted. $\mu \mathrm{g} / \mathrm{m}^{3}$, microgram per cubic meter; g, gram; $\mathrm{m}^{3}$, cubic meter; GFF, glass fiber filter; PUF, polyurethane foam; E, estimated value; - , compound not detected at a concentration above the laboratory reporting level]

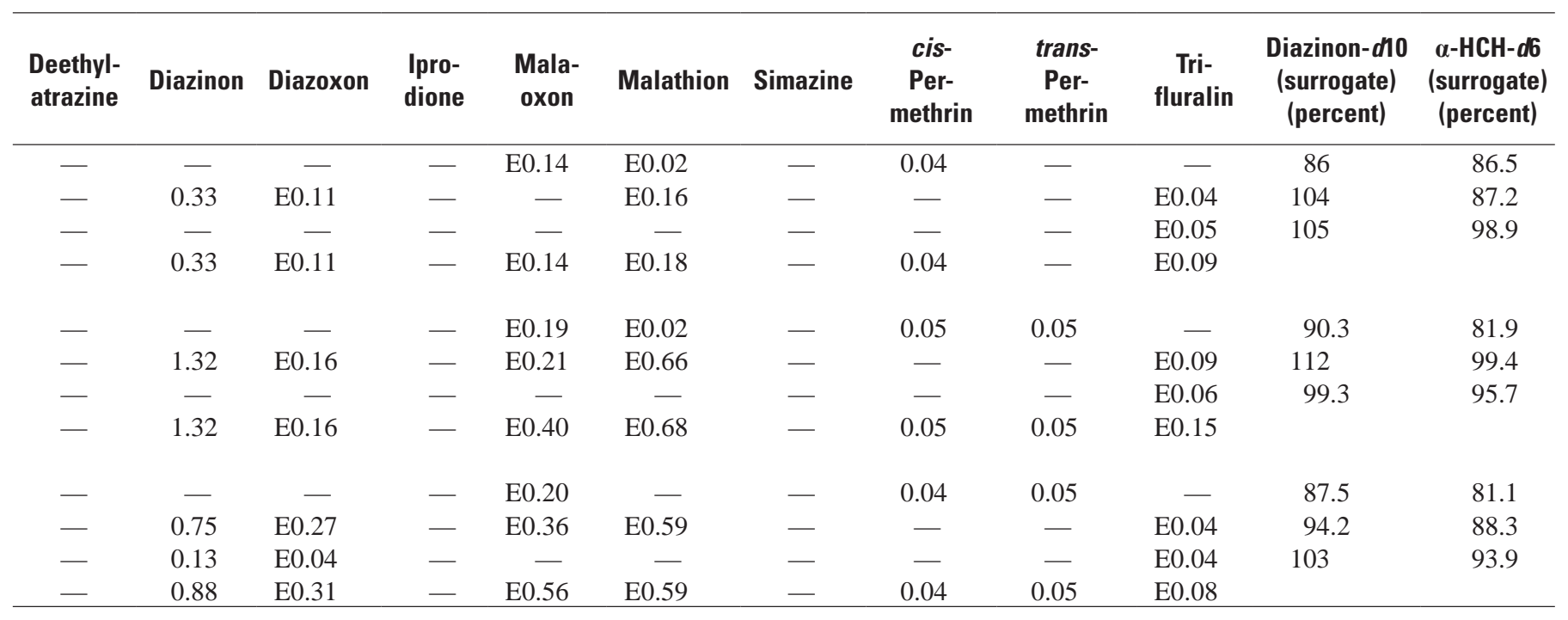


Table 14A. Analytical results for special study of trace metals in whole-water samples collected from the Sweetwater Reservoir watershed, San Diego County, California.

[See table 1 for site identification number. Time is denoted in 24-hour scale. The five digit number in parentheses below each compound name, the parameter code, is used in the U.S. Geological Survey computerized system (National Water Information System) to uniquely identify a specific constituent or property. LRL, laboratory reporting level; E, estimated value; mm/dd/yyyy, month/day/year. All values are reported as micrograms per liter unless noted. <, compound not detected at a concentration above laboratory reporting level]

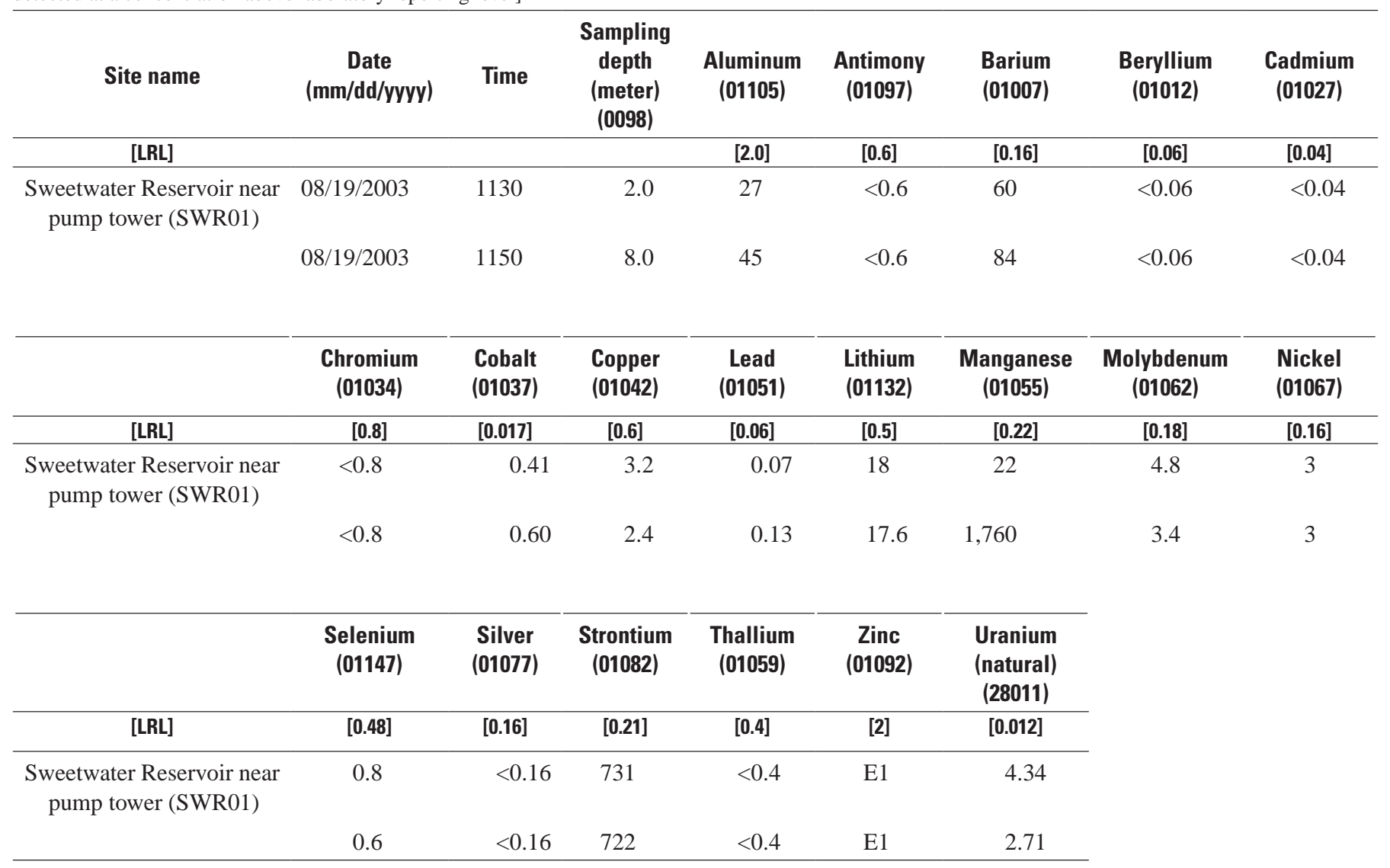


Table 14B. Analytical results for special study of trace metals in filtered-water samples collected from the Sweetwater Reservoir watershed, San Diego County, California.

[See table 1 for site identification number. Time is denoted in 24-hour scale. The five digit number in parentheses below each compound name, the parameter code, is used in the U.S. Geological Survey computerized system (National Water Information System) to uniquely identify a specific constituent or property. All values are reported as micrograms per liter unless noted. LRL, Laboratory reporting level; E, estimated value; mm/dd/yyyy, month/day/year. <, compound not detected at a concentration above laboratory reporting level]

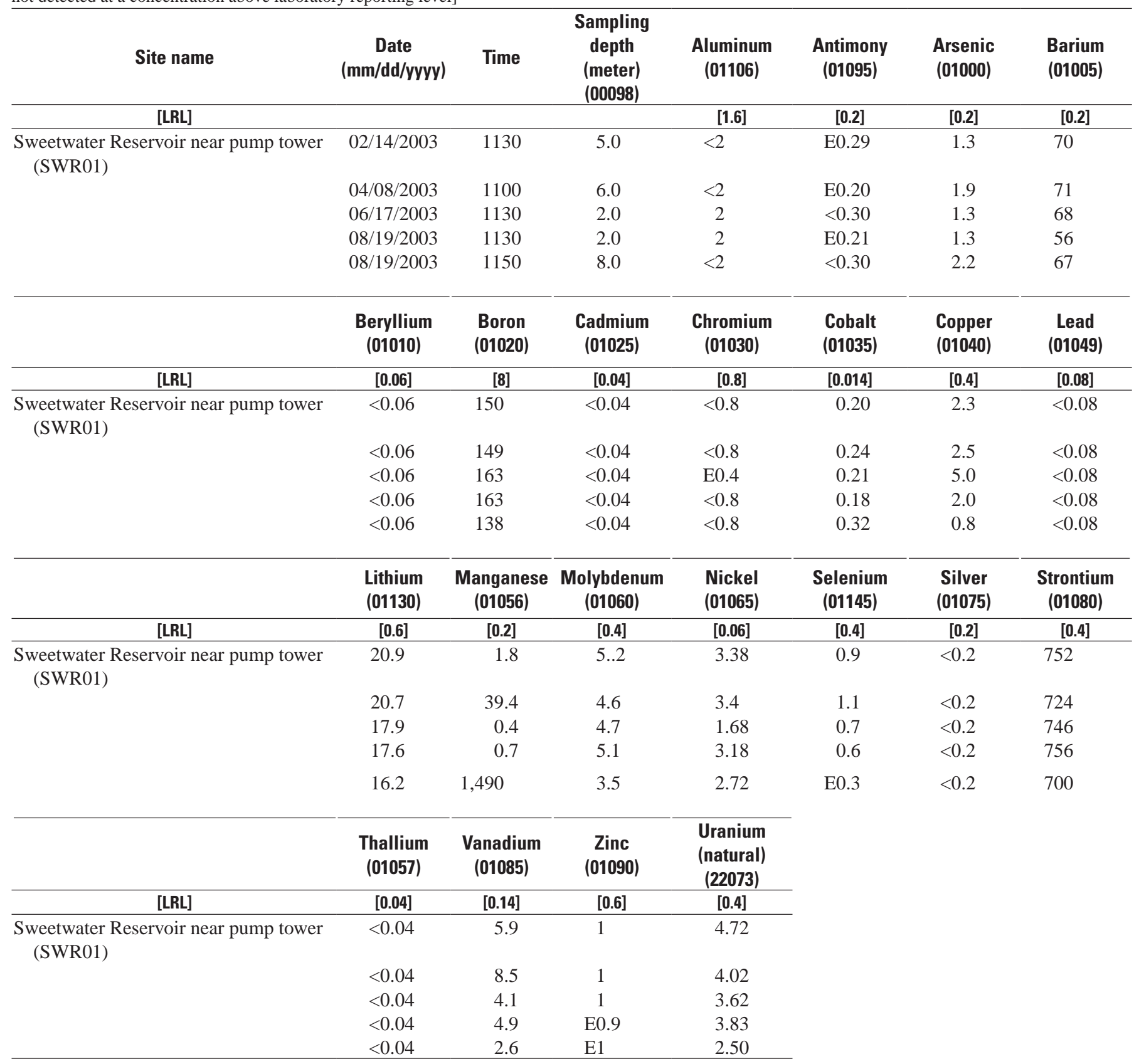


Table 15A. Anthropogenic indicator compounds analyzed in filtered-water samples collected from the Sweetwater Reservoir watershed, San Diego County, California.

[The parameter code is used in the U.S. Geological Survey computerized data system (National Water Information System) to uniquely identify a specific constituent or property. LRL, laboratory reporting level; LRL values are reported as micrograms per liter unless noted]

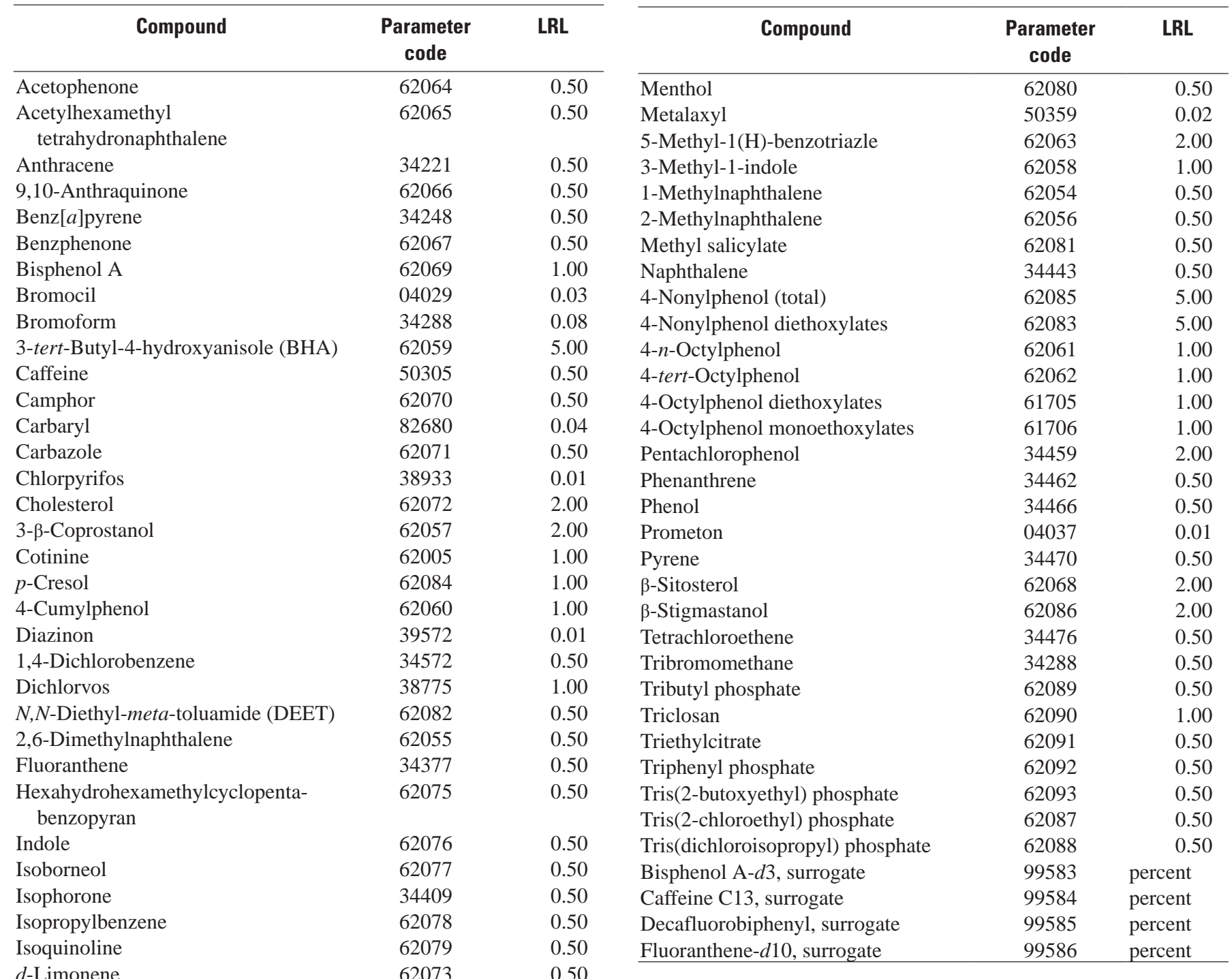


Table 15B. Analytical results from the special study of anthropogenic indicator compounds detected in filtered-water samples collected from the Sweetwater Reservoir watershed, San Diego County, California.

[The five digit number in parentheses below each compound name, the parameter code, is used in the U.S. Geological Survey computerized data system (National Water Information System) to uniquely identify a specific constituent or property. LRL, laboratory reporting level; E, estimated value; na, not applicable; - , compound not detected at a concentration above laboratory reporting level. All values are reported in micrograms per liter unless noted]

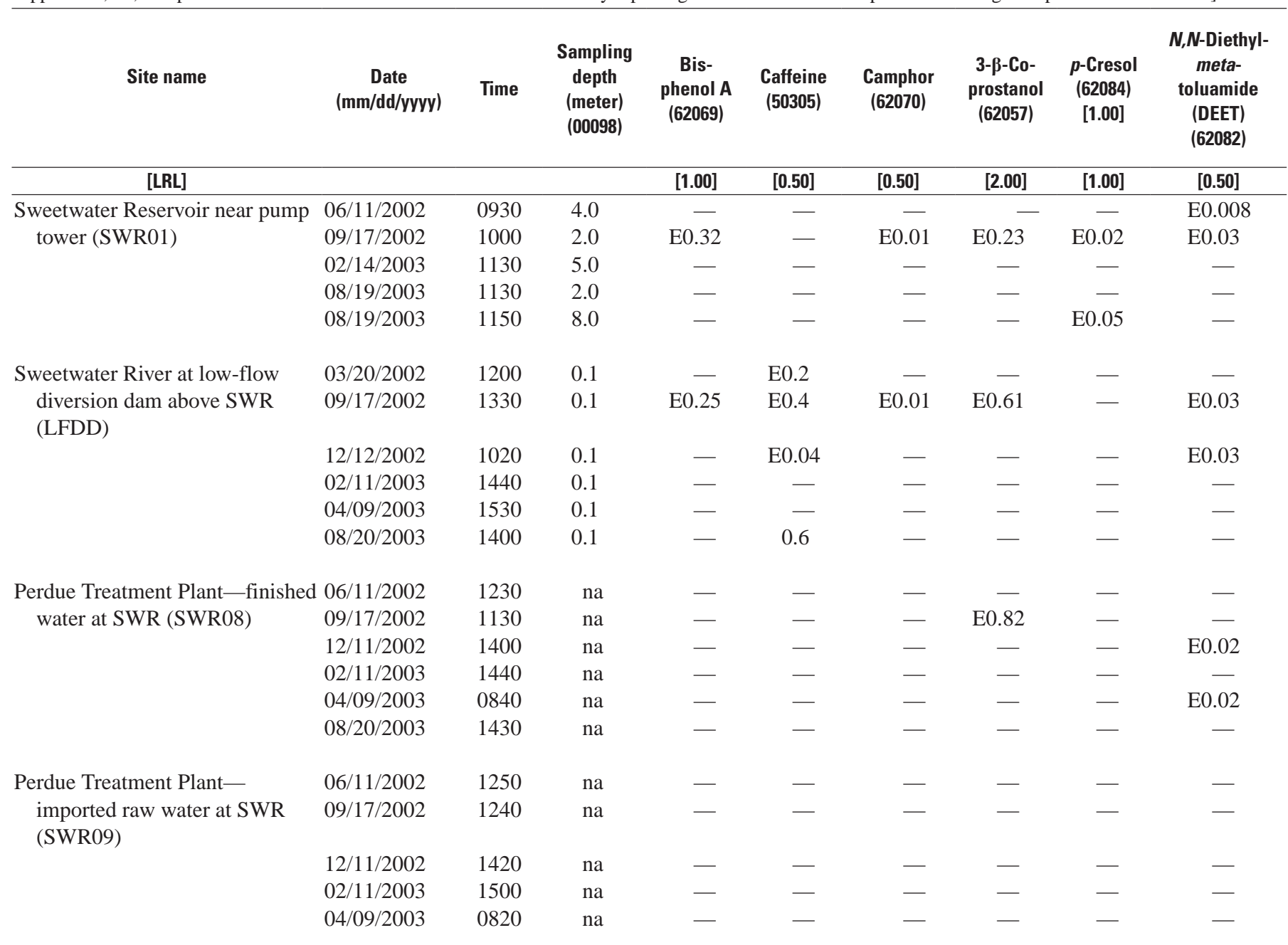


Table 15B. Analytical results from the special study of anthropogenic indicator compounds detected in filtered-water samples collected from the Sweetwater Reservoir watershed, San Diego County, California.-Continued

[The five digit number in parentheses below each compound name, the parameter code, is used in the U.S. Geological Survey computerized data system (National Water Information System) to uniquely identify a specific constituent or property. LRL, laboratory reporting level; E, estimated value; na, not applicable; - , compound not detected at a concentration above laboratory reporting level. All values are reported in micrograms per liter unless noted]

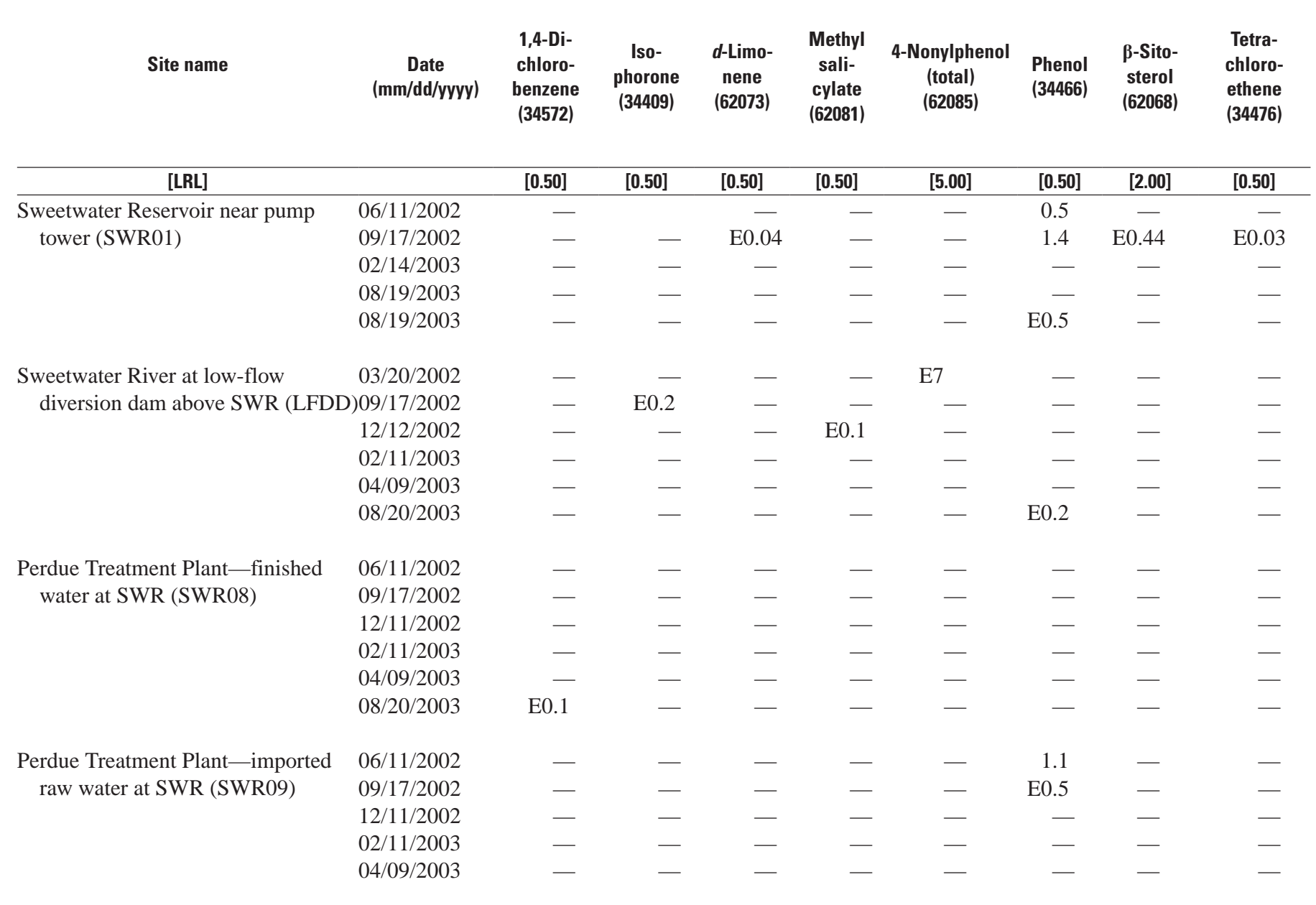


Table 15B. Analytical results from the special study of anthropogenic indicator compounds detected in filtered-water samples collected from the Sweetwater Reservoir watershed, San Diego County, California.-Continued

[The five digit number in parentheses below each compound name, the parameter code, is used in the U.S. Geological Survey computerized data system (National Water Information System) to uniquely identify a specific constituent or property. LRL, laboratory reporting level; E, estimated value; na, not applicable; - , compound not detected at a concentration above laboratory reporting level. All values are reported in micrograms per liter unless noted]

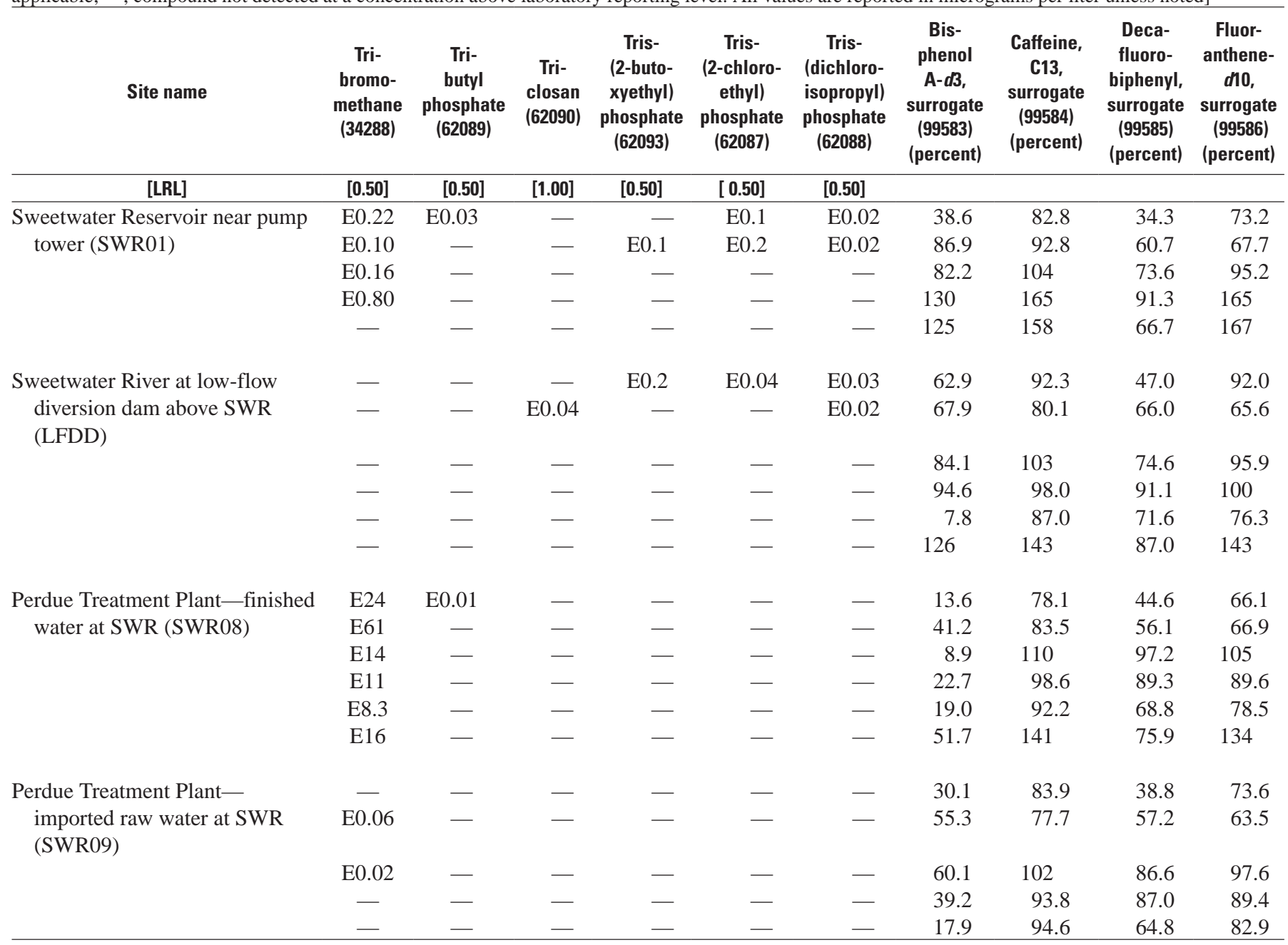




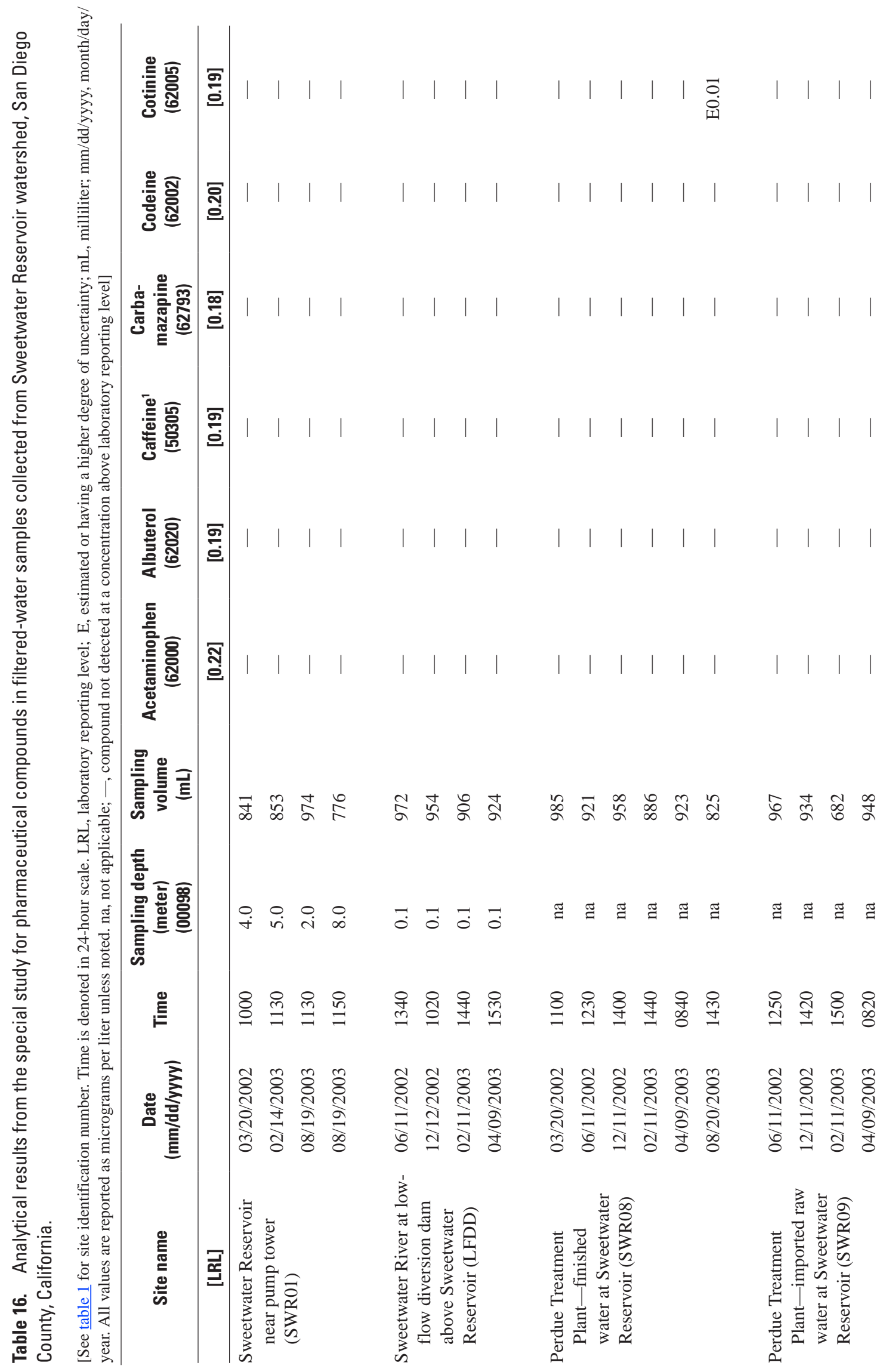




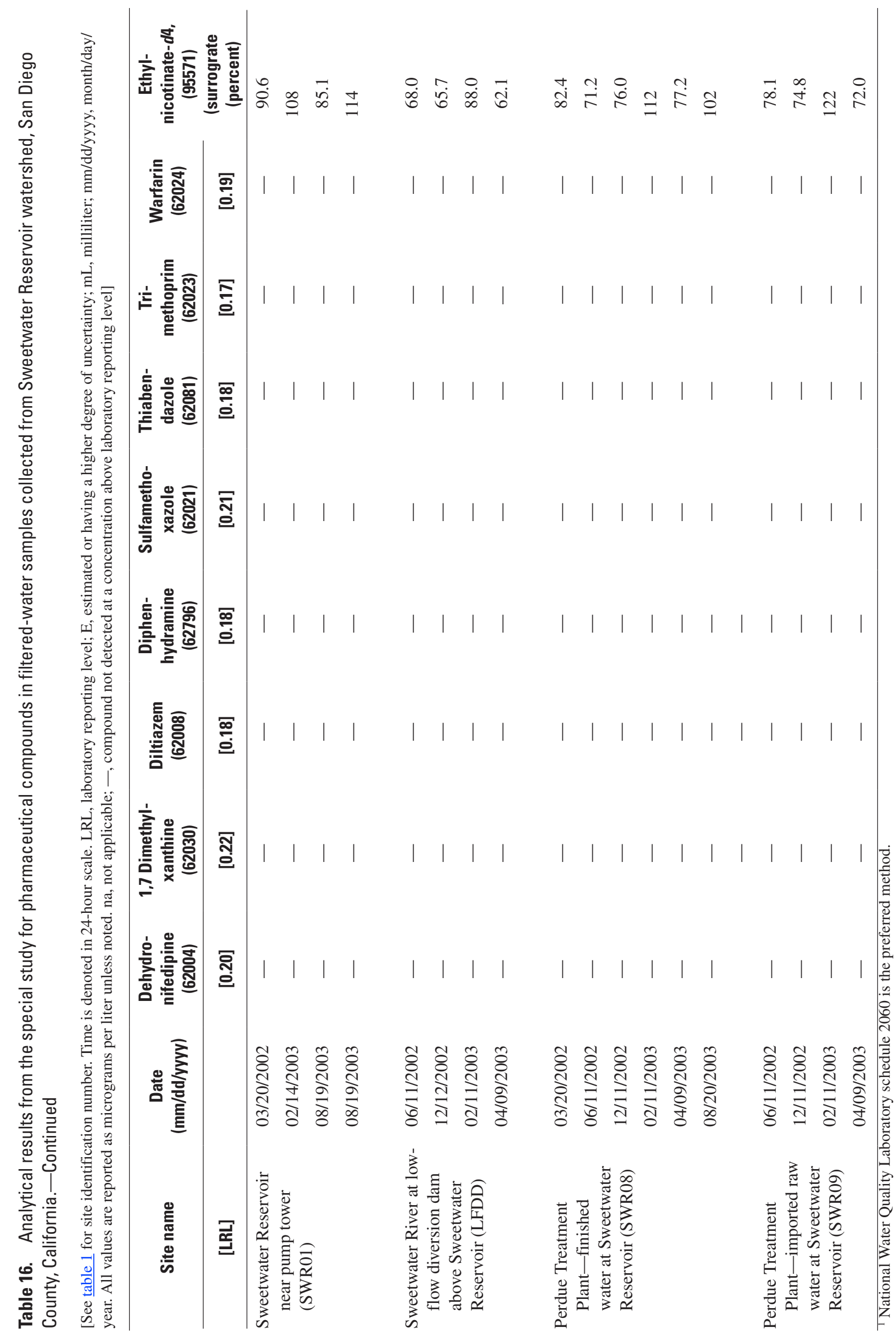




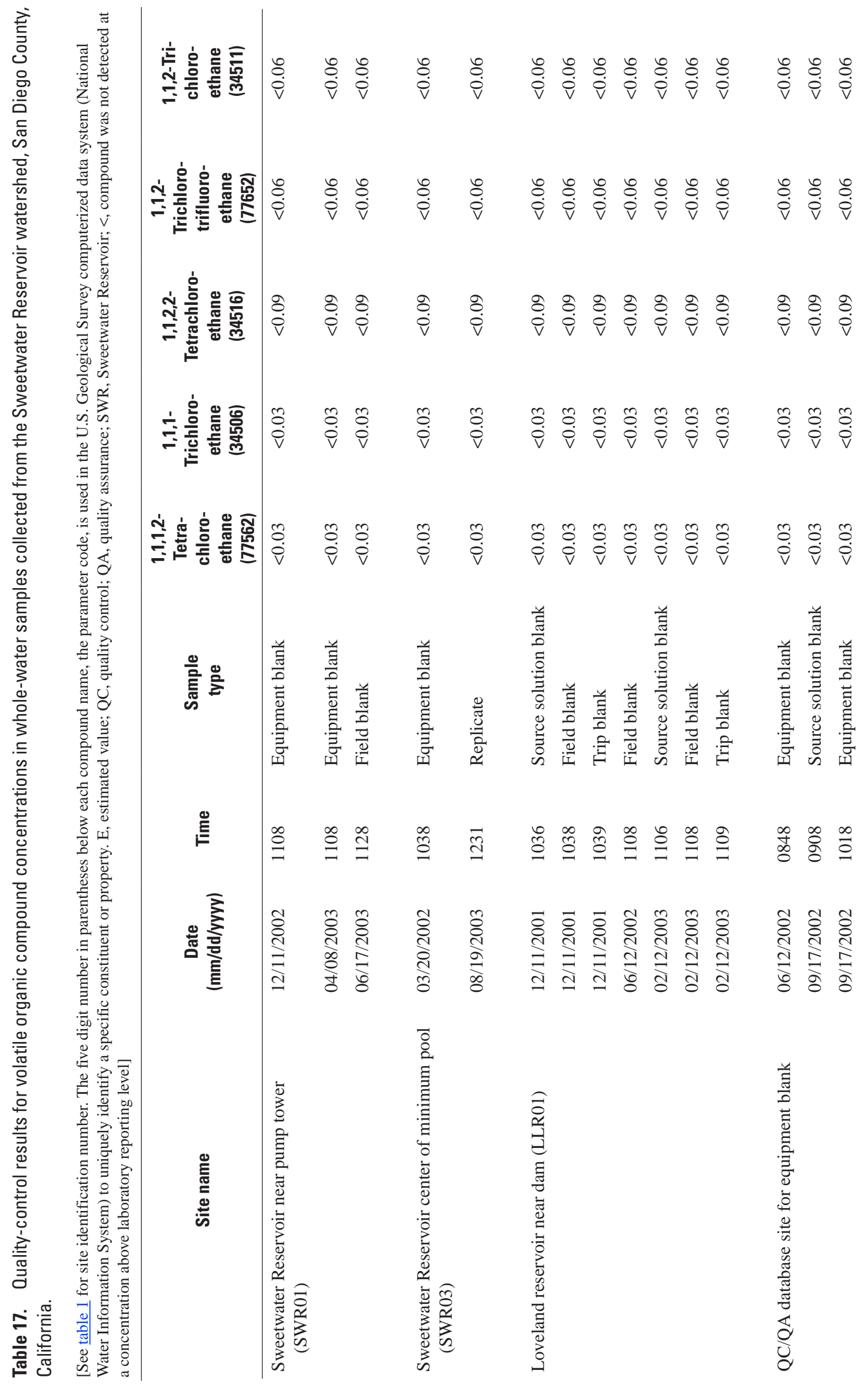




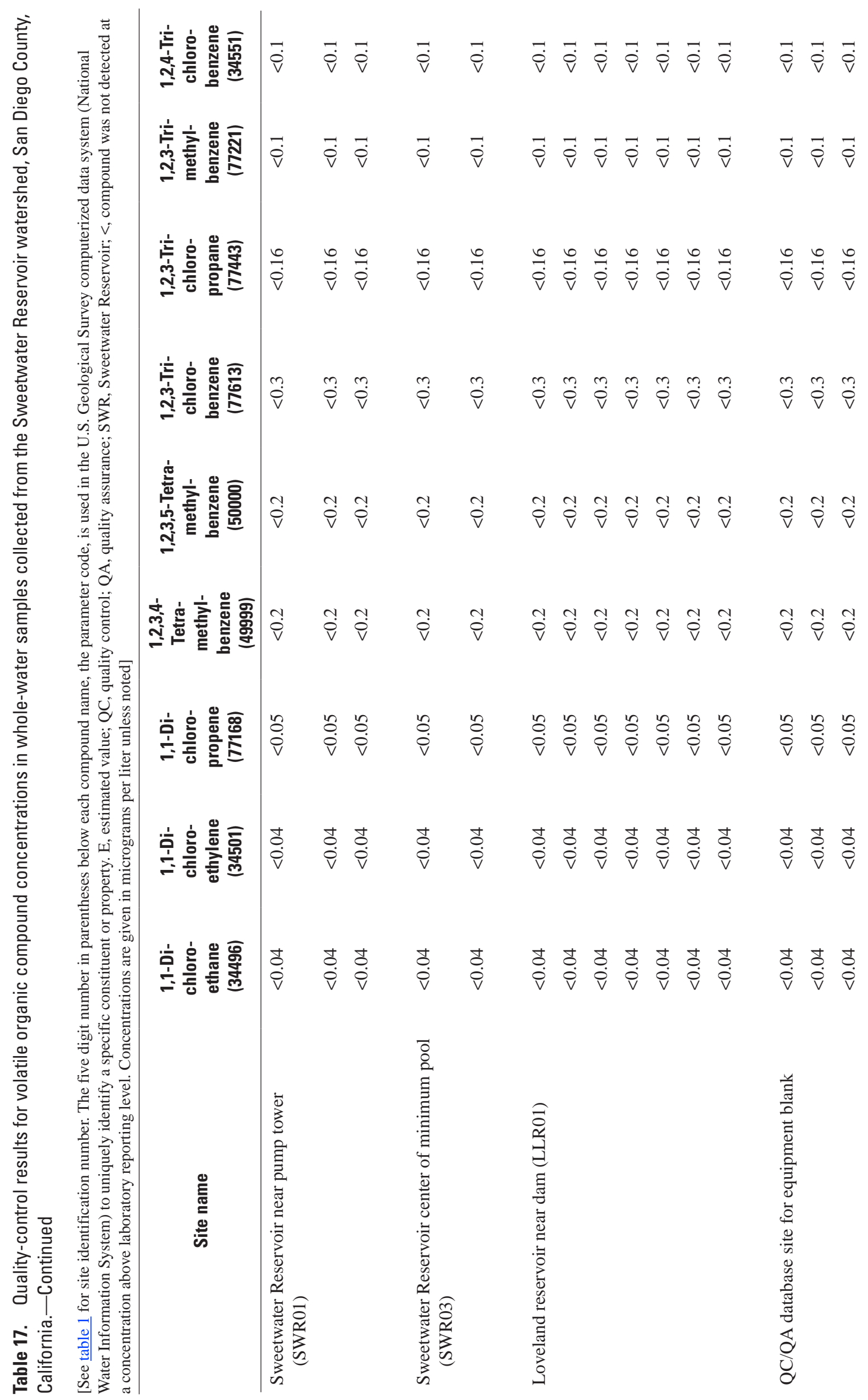




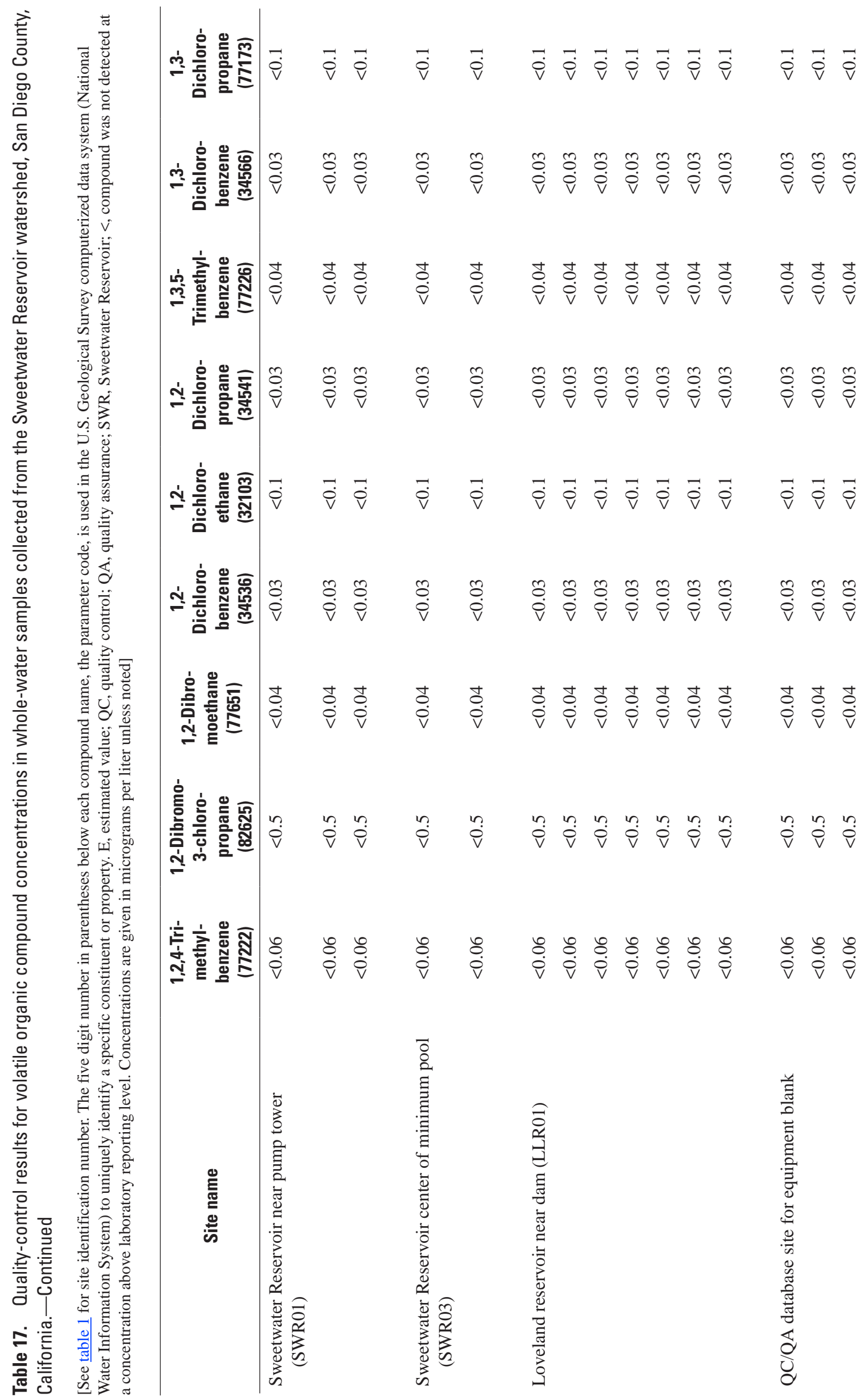




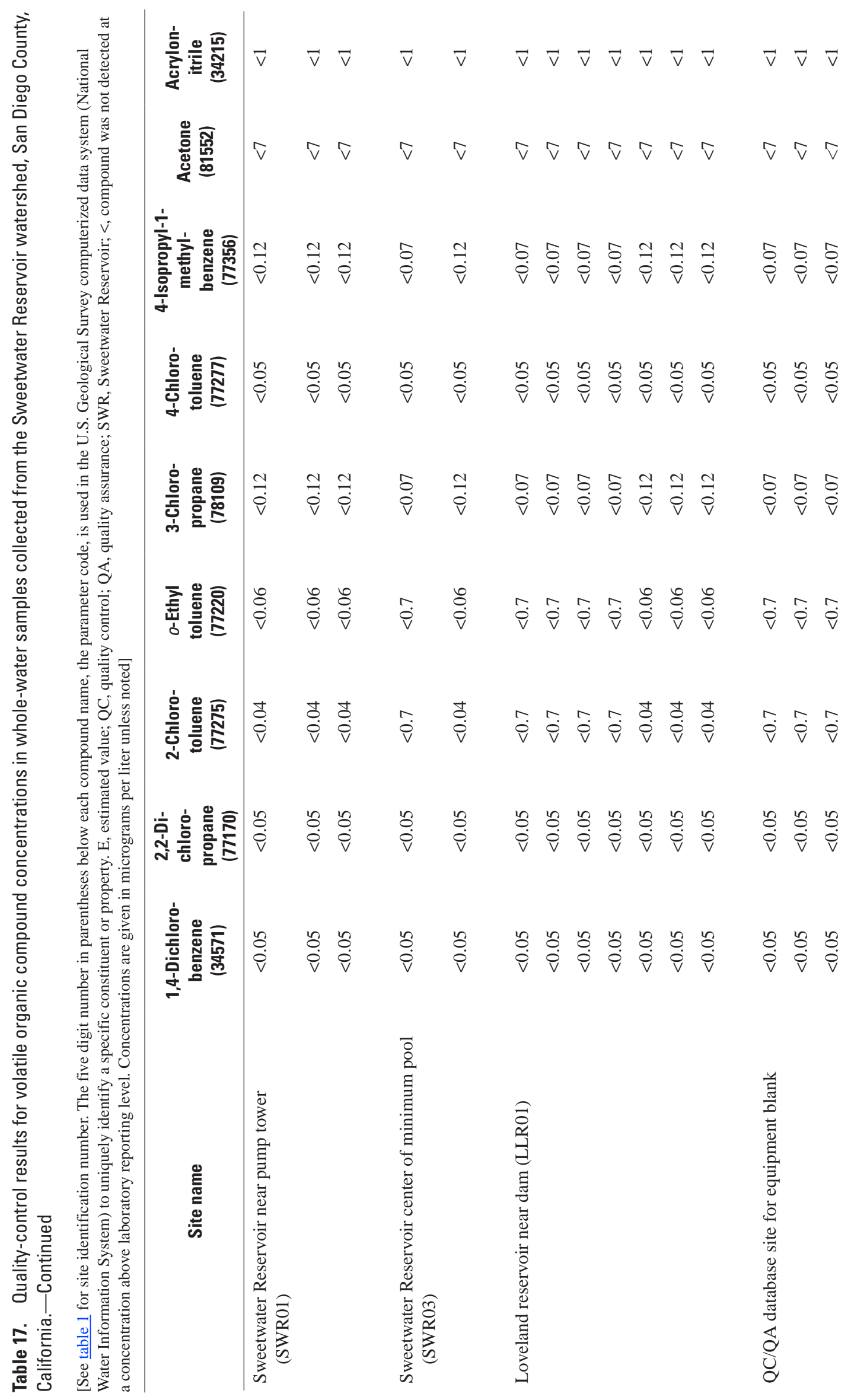




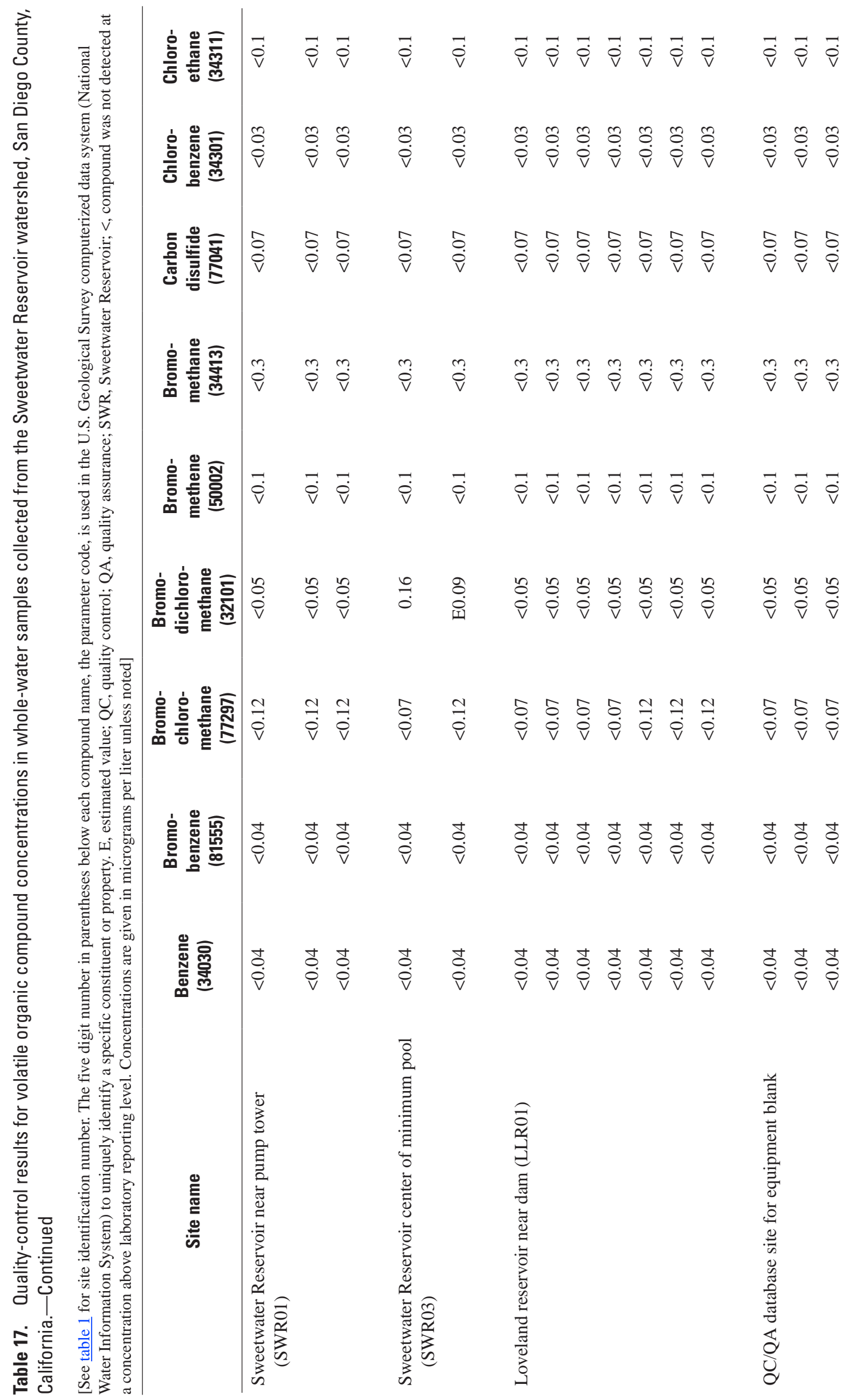




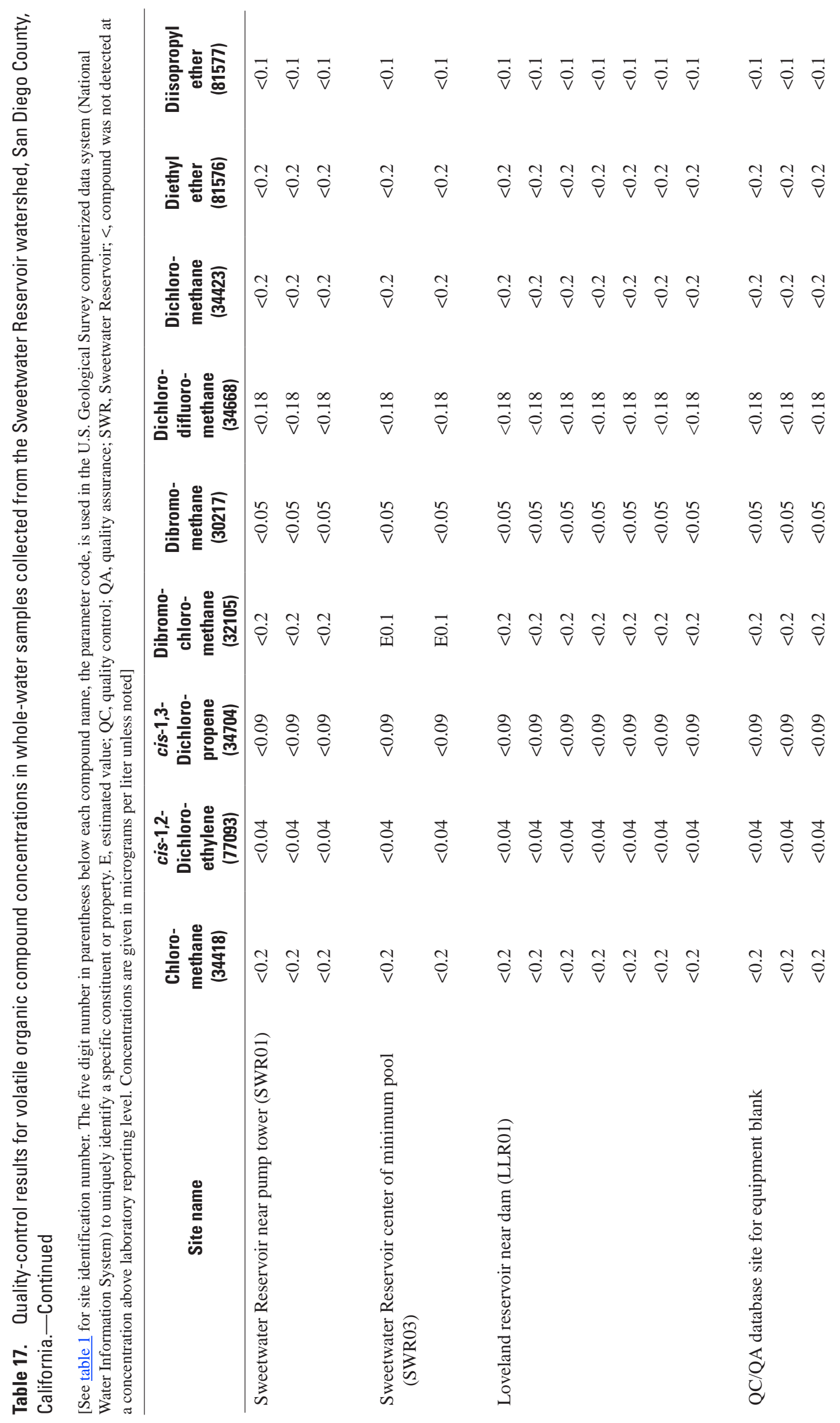




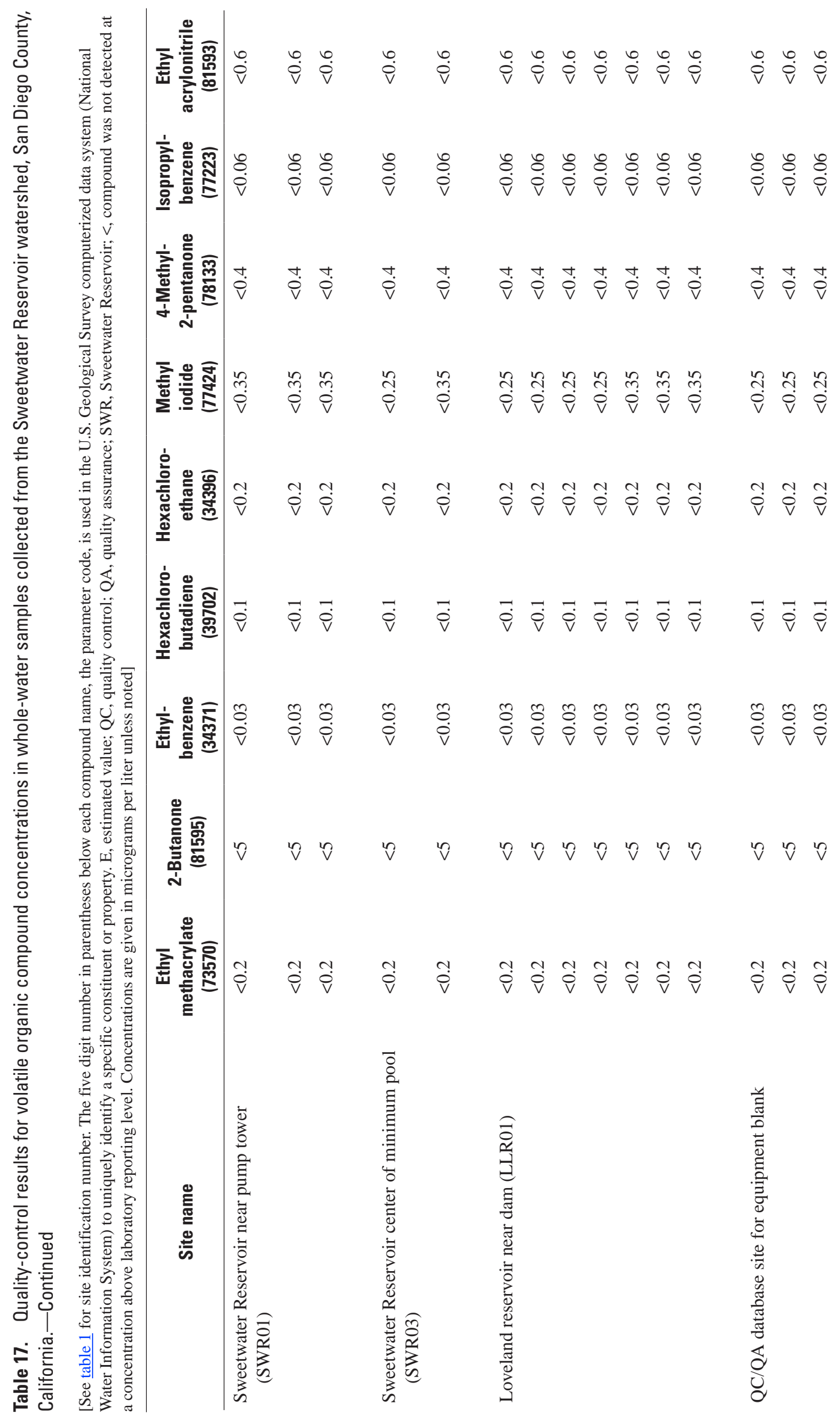




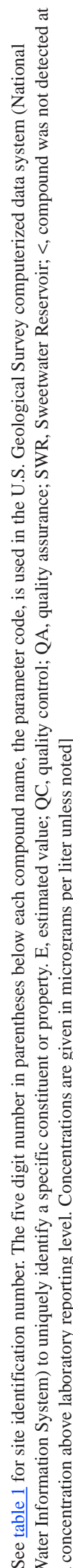

\begin{tabular}{|c|c|c|c|c|c|c|c|c|c|c|c|c|c|}
\hline 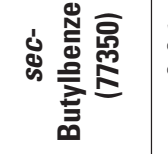 & 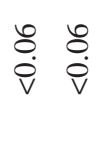 & 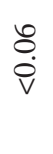 & $\stackrel{\tilde{Q}}{\dot{\varphi}}$ & $\begin{array}{l}\stackrel{\leftrightarrow}{0} \\
\dot{\theta}\end{array}$ & $\stackrel{n}{\hat{\theta}}$ & $\begin{array}{l}\hat{\sigma} \\
\hat{\theta} \\
\dot{v}\end{array}$ & $\vec{V}$ & $\begin{array}{l}0 \\
\dot{\hat{v}} \\
\dot{v}\end{array}$ & $\begin{array}{l}\stackrel{8}{\circ} \\
\dot{\vec{v}}\end{array}$ & $\begin{array}{l}\stackrel{8}{\dot{\theta}} \\
\dot{\vec{v}}\end{array}$ & $\stackrel{8}{\stackrel{\leftrightarrow}{0}}$ & $\begin{array}{l}\tilde{\theta} \\
\dot{\vec{v}}\end{array}$ & $\begin{array}{l}\hat{e} \\
\dot{\vec{v}} \\
\dot{v}\end{array}$ \\
\hline 䇋点 & $\begin{array}{l}\hat{o} \\
\dot{\dot{v}} \\
\dot{v}\end{array}$ & $\stackrel{5}{0}$ & $\stackrel{5}{0}$ & o̊. & $\stackrel{0}{0}$ & ô. & s. & ڤ్ & ¿. & o̊. & ¿̊. & $\stackrel{5}{0}$ & 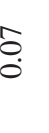 \\
\hline 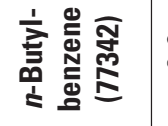 & $\begin{array}{c}\tilde{V} \\
\stackrel{\leftrightarrow}{v}\end{array}$ & $\stackrel{\sim}{\stackrel{v}{v}}$ & $\stackrel{\stackrel{\vartheta}{v}}{v}$ & $\stackrel{\tilde{Q}}{\mathrm{v}}$ & $\stackrel{\tilde{Q}}{\mathrm{v}}$ & $\begin{array}{l}\tilde{O} \\
\stackrel{\mathrm{V}}{\mathrm{V}}\end{array}$ & $\begin{array}{l}\text { Tै } \\
\stackrel{\mathrm{V}}{\mathrm{V}}\end{array}$ & 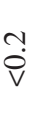 & $\stackrel{\sim}{\stackrel{v}{v}}$ & $\begin{array}{l}\tilde{Q} \\
\stackrel{\nabla}{v}\end{array}$ & $\stackrel{\tilde{v}}{\mathrm{v}}$ & $\stackrel{\stackrel{\vartheta}{v}}{\mathrm{v}}$ & $\begin{array}{l}\tilde{O} \\
\stackrel{\mathrm{V}}{\mathrm{V}}\end{array}$ \\
\hline 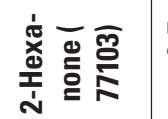 & $\hat{\dot{\theta}} \hat{\dot{v}}$ & $\hat{\dot{v}}$ & $\hat{\dot{v}}$ & $\hat{\dot{v}}$ & $\hat{\dot{v}}$ & $\hat{\dot{v}}$ & $\hat{\dot{\theta}}$ & $\hat{\dot{\theta}}$ & $\ddot{\dot{v}}$ & $\ddot{\dot{v}}$ & $\hat{\dot{v}}$ & $\hat{\dot{v}}$ & $\hat{\dot{v}}$ \\
\hline 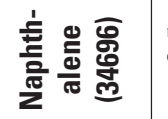 & $\begin{array}{ll}n & n \\
\dot{v} & \stackrel{v}{v}\end{array}$ & $\ddot{q}$ & $\tilde{i}$ & $\ddot{q}$ & $\ddot{q}$ & 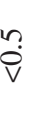 & $\ddot{q}$ & ? & $\stackrel{n}{v}$ & ? & $\ddot{q}$ & $\tilde{q}$ & $?$ \\
\hline 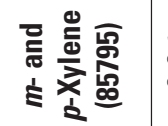 & 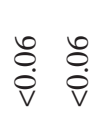 & $\stackrel{\leftrightarrow}{\dot{\varphi}}$ & $\stackrel{8}{\dot{v}}$ & $\stackrel{8}{\stackrel{8}{v}}$ & $\stackrel{8}{\dot{\varphi}}$ & $\begin{array}{l}\stackrel{8}{0} \\
\dot{\theta}\end{array}$ & $\begin{array}{l}\stackrel{8}{0} \\
\dot{\theta}\end{array}$ & 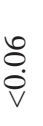 & $\begin{array}{l}\stackrel{8}{\circ} \\
\dot{0}\end{array}$ & 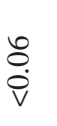 & 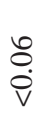 & $\stackrel{8}{\dot{\varphi}}$ & $\begin{array}{l}\stackrel{8}{0} \\
\dot{\hat{v}}\end{array}$ \\
\hline 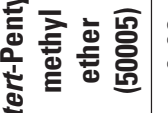 & $\begin{array}{ll}\infty & \infty \\
\stackrel{\dot{\varphi}}{0} & \stackrel{\dot{\theta}}{v}\end{array}$ & $\stackrel{\infty}{\stackrel{\infty}{\dot{P}}}$ & $\stackrel{\infty}{\stackrel{\leftrightarrow}{\dot{v}}}$ & $\stackrel{\infty}{\stackrel{0}{0}}$ & $\stackrel{\infty}{\stackrel{0}{V}}$ & $\begin{array}{l}\infty \\
\stackrel{\infty}{\circ} \\
\dot{0}\end{array}$ & $\begin{array}{l}\infty \\
\stackrel{\dot{v}}{v}\end{array}$ & $\stackrel{\infty}{\stackrel{0}{\dot{v}}}$ & $\stackrel{\infty}{\stackrel{0}{\dot{v}}}$ & $\begin{array}{l}\infty \\
\stackrel{\vec{v}}{v}\end{array}$ & $\stackrel{\infty}{\dot{\varphi}}$ & $\stackrel{\infty}{\stackrel{\leftrightarrow}{\dot{v}}}$ & $\begin{array}{l}\infty \\
\stackrel{0}{0} \\
\dot{\vec{v}}\end{array}$ \\
\hline 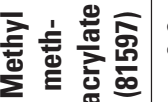 & $\stackrel{m}{\dot{v}} \stackrel{m}{\hat{v}}$ & $\stackrel{n}{v}$ & $\stackrel{m}{\mathrm{~V}}$ & $\stackrel{n}{v}$ & $\stackrel{n}{\dot{v}}$ & $\tilde{\ddot{v}}$ & $\stackrel{m}{\hat{v}}$ & $\stackrel{m}{\nabla}$ & $\stackrel{m}{v}$ & $\stackrel{n}{\vartheta}$ & $\stackrel{n}{\hat{v}}$ & $\stackrel{n}{v}$ & $\stackrel{m}{\hat{v}}$ \\
\hline
\end{tabular}

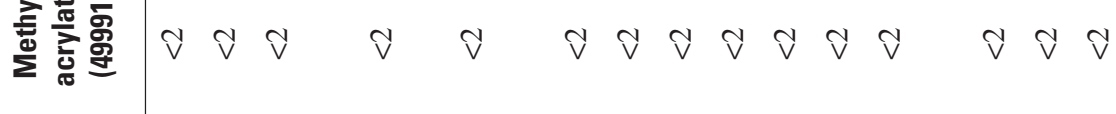
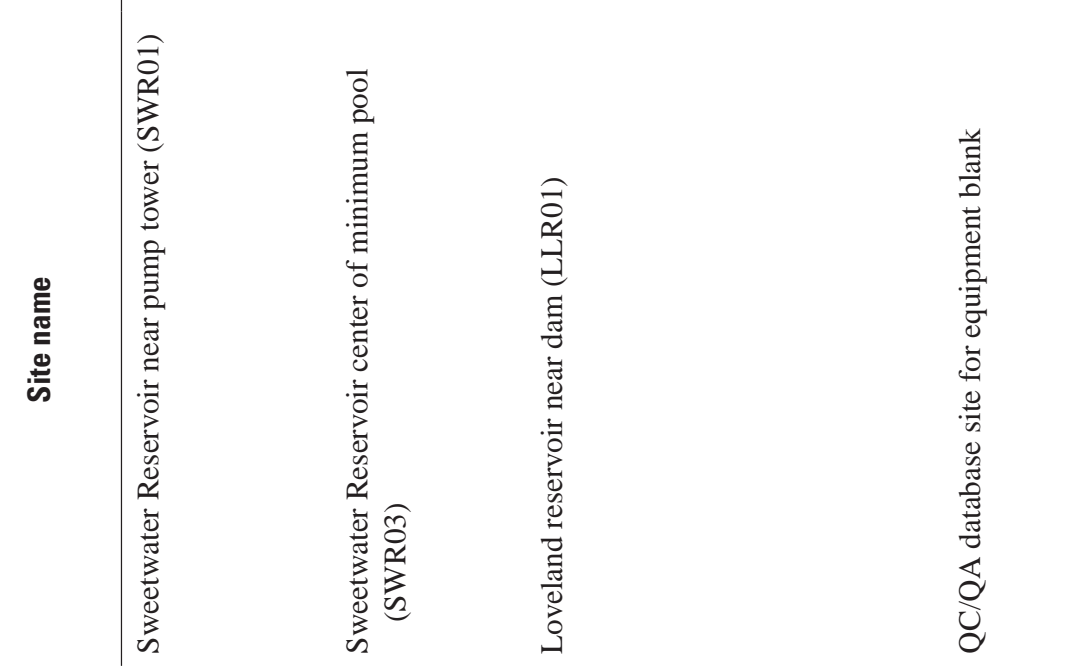


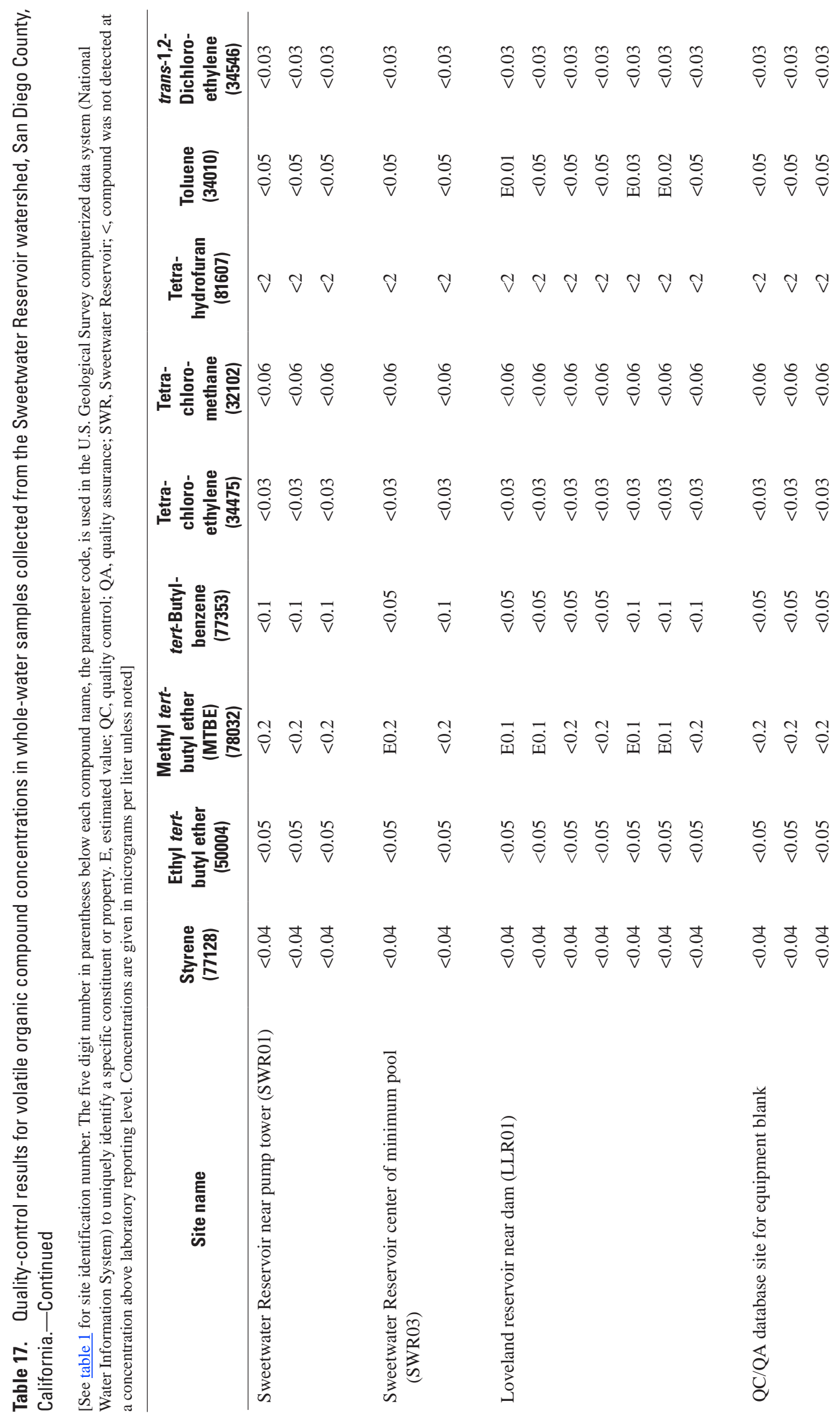




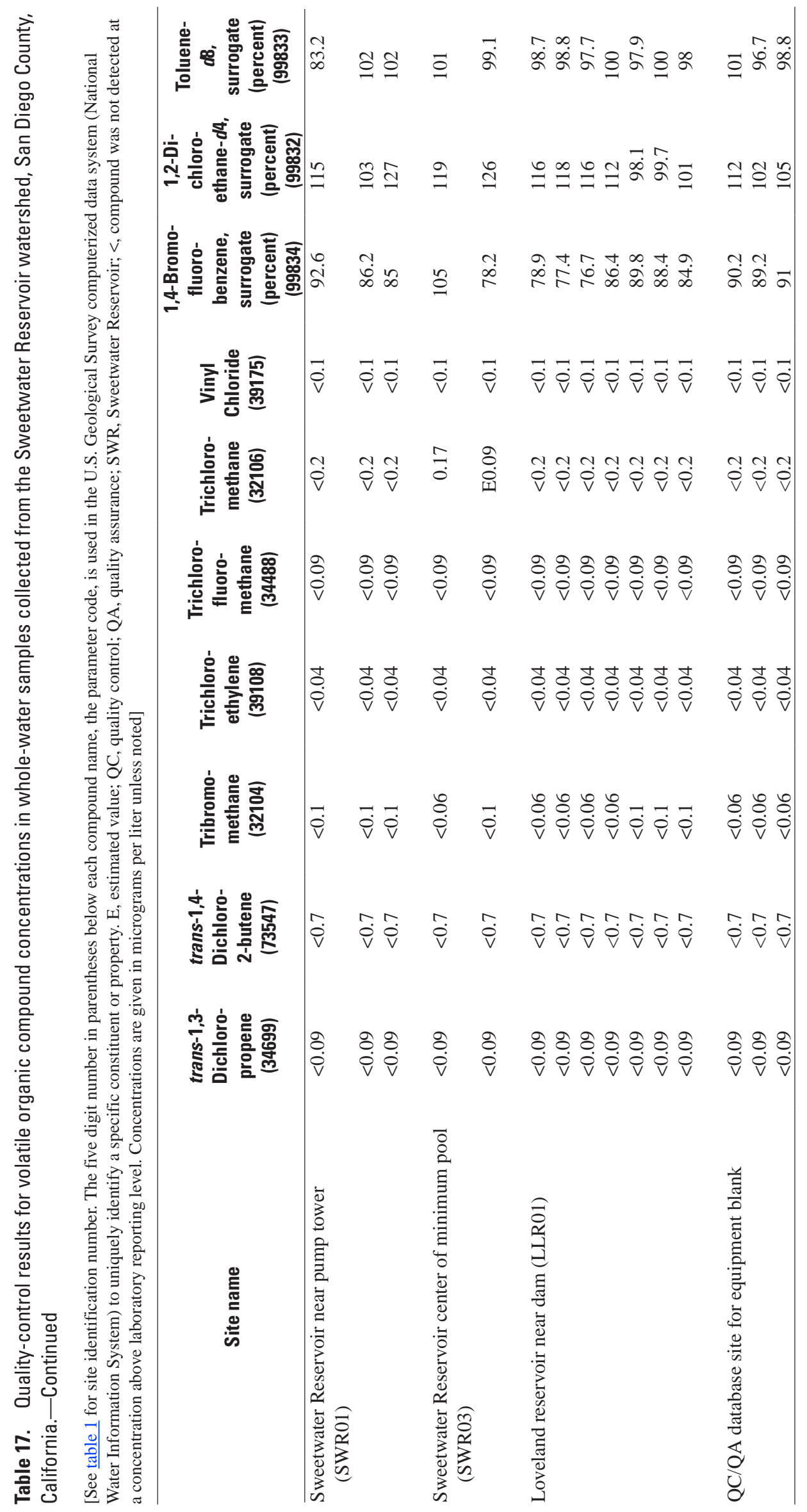


Table 18. Quality-control results for pesticide concentrations in filtered-water samples, analyzed using U.S. Geological Survey's National Water Quality Laboratory schedule 2001, from Loveland Reservoir, San Diego County, California.

[See table 1 for site identification number. Time is denoted in 24-hour scale. The five digit number in parentheses below the compound name, the parameter code, is used in the U.S. Geological Survey's computerized data system (National Water Information System) to uniquely identify a specific constituent or property. <, compound not detected at a concentration above laboratory reporting limit; na, not applicable. Concentrations are given in micrograms per liter unless noted]

\begin{tabular}{|c|c|c|c|c|c|c|c|}
\hline Site name & $\begin{array}{c}\text { Date } \\
\text { (mm/dd/yyyy) }\end{array}$ & Time & $\begin{array}{l}\text { Sample } \\
\text { type }\end{array}$ & $\begin{array}{c}\text { Sampling } \\
\text { depth } \\
\text { (00098) } \\
\text { (meter) }\end{array}$ & $\begin{array}{c}\text { Acetochlor } \\
\text { (49260) }\end{array}$ & $\begin{array}{c}\text { Alachlor } \\
\text { (46342) }\end{array}$ & $\begin{array}{c}\text { Atrazine } \\
\text { (39632) }\end{array}$ \\
\hline Loveland Reservoir near dam (LLR01) & $06 / 12 / 2002$ & 1121 & Replicate & 2.0 & $<0.006$ & $<0.004$ & $<0.007$ \\
\hline QA/QC database site for equipment blank & $02 / 11 / 2003$ & 1538 & Equipment blank & na & $<0.006$ & $<0.004$ & $<0.007$ \\
\hline Site name & $\begin{array}{c}\text { Azinphos-methyl } \\
\text { (82686) }\end{array}$ & $\begin{array}{l}\text { Benfluralin } \\
(\mathbf{8 2 6 7 3 )}\end{array}$ & $\begin{array}{c}\text { Butylate } \\
\text { (04028) }\end{array}$ & $\begin{array}{l}\text { Carbaryl } \\
(\mathbf{8 2 6 8 0})\end{array}$ & $\begin{array}{l}\text { Carbofuran } \\
\text { (82674) }\end{array}$ & $\begin{array}{l}\text { Chlor- } \\
\text { pyrifos } \\
\text { (38933) }\end{array}$ & $\begin{array}{l}\text { Cyanazine } \\
\text { (04041) }\end{array}$ \\
\hline \multirow[t]{2}{*}{ Loveland reservoir near dam (LLR01) } & $<0.05$ & $<0.01$ & $<0.002$ & $<0.041$ & $<0.02$ & $<0.005$ & $<0.018$ \\
\hline & $<0.05$ & $<0.01$ & $<0.002$ & $<0.041$ & $<0.02$ & $<0.005$ & $<0.018$ \\
\hline QA/QC database site for equipment blank & $<0.05$ & $<0.01$ & $<0.002$ & $<0.041$ & $<0.02$ & $<0.005$ & $<0.018$ \\
\hline Site name & $\begin{array}{l}\text { Dacthal } \\
\text { (DCPA) } \\
\text { (82682) } \\
\end{array}$ & $\begin{array}{c}p, p^{\prime}-\mathrm{DDE} \\
(34653)\end{array}$ & $\begin{array}{l}\text { Deethyl- } \\
\text { atrazine } \\
\text { (04040) } \\
\end{array}$ & $\begin{array}{c}\text { Diazinon } \\
\text { (39572) }\end{array}$ & $\begin{array}{l}\text { Dieldrin } \\
\text { (39381) }\end{array}$ & $\begin{array}{c}\text { 2,6-Di- } \\
\text { ethylaniline } \\
(82660)\end{array}$ & $\begin{array}{l}\text { Disulfoton } \\
\text { (82677) }\end{array}$ \\
\hline Site name & $\begin{array}{l}\text { EPTC } \\
\text { (82668) }\end{array}$ & $\begin{array}{l}\text { Ethal- } \\
\text { fluralin } \\
\text { (82663) }\end{array}$ & $\begin{array}{l}\text { Ethoprophos } \\
\text { (82672) }\end{array}$ & $\begin{array}{l}\text { Fonofos } \\
\text { (04095) }\end{array}$ & $\begin{array}{l}\alpha-\mathrm{HCH} \\
(34253)\end{array}$ & $\begin{array}{l}\text { Lindane } \\
\text { (39341) }\end{array}$ & $\begin{array}{l}\text { Linuron } \\
(82666)\end{array}$ \\
\hline \multirow[t]{2}{*}{ Loveland reservoir near dam (LLR01) } & $<0.005$ & $<0.009$ & $<0.005$ & $<0.003$ & $<0.005$ & $<0.004$ & $<0.035$ \\
\hline & $<0.002$ & $<0.009$ & $<0.005$ & $<0.003$ & $<0.005$ & $<0.004$ & $<0.035$ \\
\hline QA/QC database site for equipment blank & $<0.002$ & $<0.009$ & $<0.005$ & $<0.003$ & $<0.005$ & $<0.004$ & $<0.035$ \\
\hline Site name & $\begin{array}{c}\text { Malathion } \\
\text { (39532) }\end{array}$ & $\begin{array}{l}\text { Metolachlor } \\
\text { (39415) }\end{array}$ & $\begin{array}{l}\text { Metribuzin } \\
\text { (82630) }\end{array}$ & $\begin{array}{l}\text { Molinate } \\
\text { (82671) }\end{array}$ & $\begin{array}{c}\text { Napropamide } \\
\text { (82684) }\end{array}$ & $\begin{array}{l}\text { Parathion } \\
\text { (39542) }\end{array}$ & $\begin{array}{l}\text { Para- } \\
\text { thion- } \\
\text { methyl } \\
(82667) \\
\end{array}$ \\
\hline Loveland reservoir near dam (LLR01) & $<0.027$ & $<0.013$ & $<0.006$ & $<0.002$ & $<0.007$ & $<0.007$ & $<0.006$ \\
\hline Site name & $\begin{array}{l}\text { Propargite } \\
\text { (82685) }\end{array}$ & $\begin{array}{c}\text { Propy- } \\
\text { zamide (82676) } \\
\end{array}$ & $\begin{array}{c}\text { Simazine } \\
(04035)\end{array}$ & $\begin{array}{c}\text { Tebuthiuron } \\
(82670)\end{array}$ & $\begin{array}{c}\text { Terbacil } \\
(82665)\end{array}$ & $\begin{array}{c}\text { Terbufos } \\
\text { (82675) }\end{array}$ & $\begin{array}{c}\text { Thiobencarb } \\
\text { (82681) }\end{array}$ \\
\hline \multirow[t]{2}{*}{ Loveland reservoir near dam (LLR01) } & $<0.02$ & $<0.004$ & $<0.011$ & $<0.02$ & $<0.034$ & $<0.02$ & $<0.005$ \\
\hline & $<0.02$ & $<0.004$ & $<0.009$ & $<0.02$ & $<0.034$ & $<0.02$ & $<0.005$ \\
\hline QA/QC database site for equipment blank & $<0.02$ & $<0.004$ & $<0.005$ & $<0.02$ & $<0.034$ & $<0.02$ & $<0.005$ \\
\hline Site name & $\begin{array}{l}\text { Triallate } \\
\text { (82678) }\end{array}$ & $\begin{array}{l}\text { Trifluralin } \\
\text { (82661) }\end{array}$ & $\begin{array}{c}\text { Diazinon-d10, } \\
\text { surrogate } \\
\text { (91063) } \\
\text { (percent) }\end{array}$ & $\begin{array}{c}\alpha-\mathrm{HCH}-d 6, \\
\text { surrogate } \\
\text { (91065) } \\
\text { (percent) }\end{array}$ & & & \\
\hline \multirow[t]{2}{*}{ Loveland reservoir near dam (LLR01) } & $<0.002$ & $<0.009$ & 90.1 & 82.3 & & & \\
\hline & $<0.002$ & $<0.009$ & 103 & 87.0 & & & \\
\hline QA/QC database site for equipment blank & $<0.002$ & $<0.009$ & 104 & 103 & & & \\
\hline
\end{tabular}


Table 19. Quality-control results for pesticide concentrations in filtered-water samples, analyzed using U.S. Geological Survey's National Water Quality Laboratory schedule 2060, from Sweetwater Reservoir, San Diego County, California.

[See table 1 for site identification numbers. Time is denoted in 24 hour scale. The number below each compound is the parameter code used in the U.S. Geological Survey computerized data system (National Water Information system) to uniquely identify a specific constituent or property. QC, quality control; QA, quality assurance; na, not applicable; <, compound not detected at a concentration above laboratory reporting level. Concentrations are given in micrograms per liter unless noted]

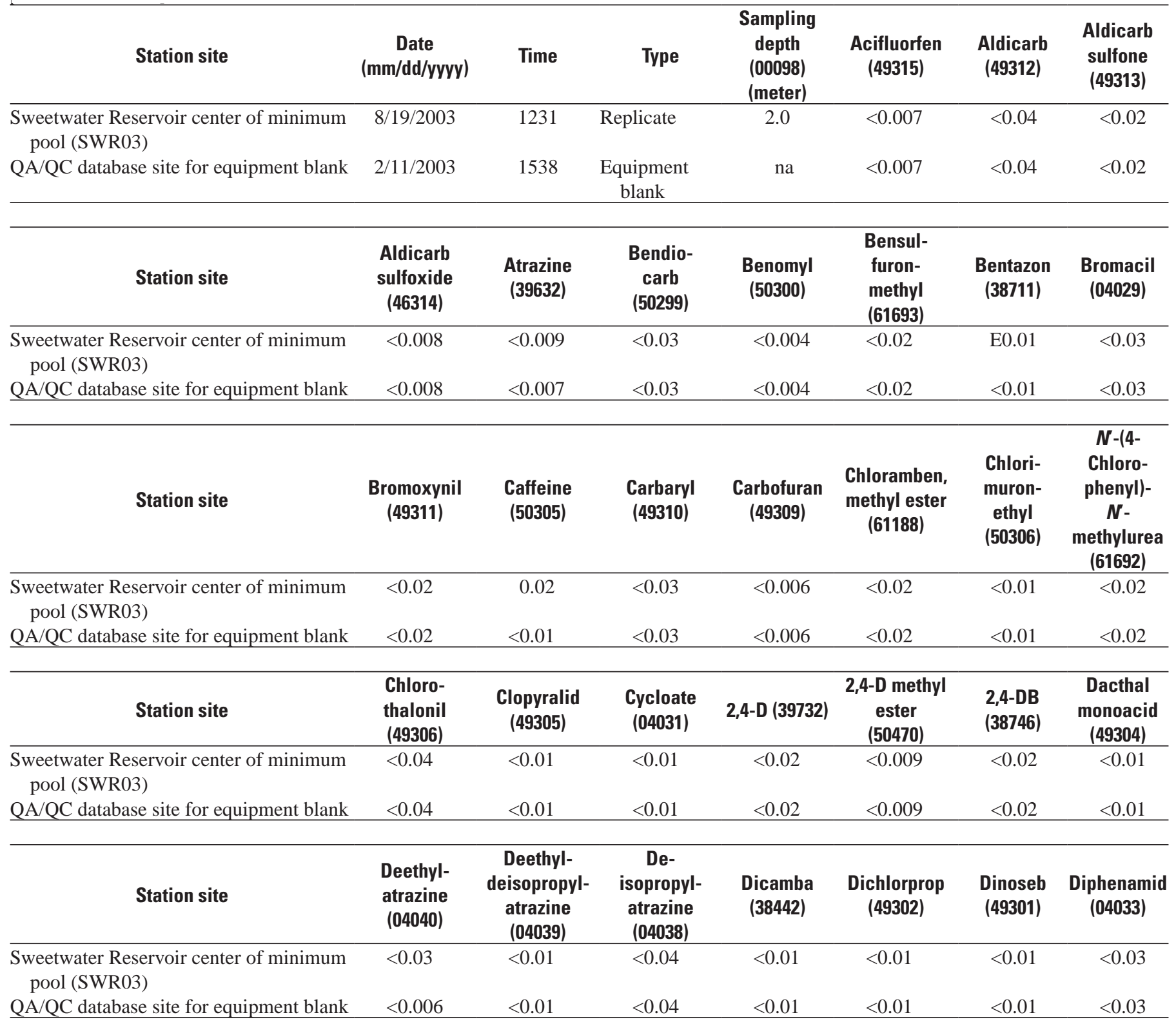


Table 19. Quality-control results for pesticide concentrations in filtered-water samples, analyzed using U.S. Geological Survey's National Water Quality Laboratory schedule 2060, from Sweetwater Reservoir, San Diego County, California._-Continued

[See table 1 for site identification numbers. Time is denoted in 24 hour scale. The number below each compound is the parameter code used in the U.S. Geological Survey computerized data system (National Water Information system) to uniquely identify a specific constituent or property. QC, quality control; QA, quality assurance; na, not applicable; <, compound not detected at a concentration above laboratory reporting level. Concentrations are given in micrograms per liter unless noted]

\begin{tabular}{|c|c|c|c|c|c|c|c|}
\hline Station site & $\begin{array}{l}\text { Diuron } \\
(49300)\end{array}$ & $\begin{array}{c}\text { Fenuron } \\
\text { (49297) }\end{array}$ & $\begin{array}{l}\text { Flumetsulam } \\
\qquad(61694)\end{array}$ & $\begin{array}{c}\text { Fluo- } \\
\text { meturon } \\
\text { (38811) }\end{array}$ & $\begin{array}{l}\text { Hydroxy- } \\
\text { atrazine } \\
(50355) \\
\end{array}$ & $\begin{array}{c}\text { 3-Hydroxy } \\
\text { carbofuran } \\
(49308)\end{array}$ & $\begin{array}{c}\text { Imazaquin } \\
(50356)\end{array}$ \\
\hline $\begin{array}{l}\text { Sweetwater Reservoir center of minimum } \\
\text { pool (SWR03) }\end{array}$ & 0.03 & $<0.03$ & $<0.01$ & $<0.03$ & $<0.008$ & $<0.006$ & $<0.02$ \\
\hline \multirow[t]{2}{*}{ QA/QC database site for equipment blank } & $<0.01$ & $<0.03$ & $<0.01$ & $<0.03$ & $<0.008$ & $<0.006$ & $<0.02$ \\
\hline & $\begin{array}{c}\text { Imazethapyr } \\
\text { (50407) }\end{array}$ & $\begin{array}{c}\text { Imidacloprid } \\
(61695)\end{array}$ & $\begin{array}{c}3-K e t o- \\
\text { carbofuran } \\
(50295) \\
\end{array}$ & $\begin{array}{l}\text { Linuron } \\
\text { (38478) }\end{array}$ & $\begin{array}{l}\text { MCPA } \\
(38482)\end{array}$ & $\begin{array}{l}\text { МCPB } \\
(38487)\end{array}$ & $\begin{array}{c}\text { Metalaxyl } \\
\text { (50359) }\end{array}$ \\
\hline $\begin{array}{l}\text { Sweetwater Reservoir center of minimum } \\
\text { pool (SWR03) }\end{array}$ & $<0.02$ & $<0.007$ & $<1.5$ & $<0.01$ & $<0.02$ & $<0.01$ & $<0.02$ \\
\hline \multirow[t]{2}{*}{ QA/QC database site for equipment blank } & $<0.02$ & $<0.007$ & $<1.5$ & $<0.01$ & $<0.02$ & $<0.01$ & $<0.02$ \\
\hline & $\begin{array}{c}\text { Methiocarb } \\
\text { (38501) }\end{array}$ & $\begin{array}{l}\text { Methomyl } \\
\text { (49296) }\end{array}$ & $\begin{array}{c}\text { Met- } \\
\text { sulfuron } \\
\text { methyl } \\
\text { (61697) }\end{array}$ & $\begin{array}{l}\text { Neburon } \\
\text { (49294) }\end{array}$ & $\begin{array}{l}\text { Nicosulfuron } \\
\text { (50364) }\end{array}$ & $\begin{array}{c}\text { Nor- } \\
\text { flurazon } \\
\text { (49293) }\end{array}$ & $\begin{array}{l}\text { Oryzalin } \\
\text { (49292) }\end{array}$ \\
\hline \multirow[t]{2}{*}{ QA/QC database site for equipment blank } & $<0.008$ & $<0.004$ & $<0.03$ & $<0.01$ & $<0.01$ & $<0.02$ & $<0.02$ \\
\hline & $\begin{array}{l}\text { Oxamyl } \\
\text { (38866) }\end{array}$ & $\begin{array}{l}\text { Picloram } \\
\text { (49291) }\end{array}$ & $\begin{array}{l}\text { Propham } \\
\text { (49236) }\end{array}$ & $\begin{array}{l}\text { Propi- } \\
\text { conazole } \\
(50471)\end{array}$ & $\begin{array}{l}\text { Propoxur } \\
(38538)\end{array}$ & $\begin{array}{l}\text { Siduron } \\
\text { (38548) }\end{array}$ & $\begin{array}{c}\text { Sulfo- } \\
\text { meturon- } \\
\text { methyl } \\
\text { (50337) }\end{array}$ \\
\hline $\begin{array}{l}\text { Sweetwater Reservoir center of minimum } \\
\text { pool (SWR03) }\end{array}$ & $<0.01$ & $<0.02$ & $<0.01$ & $<0.02$ & $<0.008$ & $<0.02$ & $<0.009$ \\
\hline QA/QC database site for equipment blank & $<0.01$ & $<0.02$ & $<0.01$ & $<0.02$ & $<0.008$ & $<0.02$ & $<0.009$ \\
\hline
\end{tabular}


Table 20A. Quality-control replicate results for volatile organic compounds with low breakthrough volumes from the Sweetwater Reservoir atmospheric site, San Diego County, California.

[The site identification number is 324141117001601 . Concentrations are given in parts per billion by volume (ppbv). Time is denoted in 24 -hour scale. ${ }^{\circ} \mathrm{C}$, degree Celsius; $\mathrm{mm}$ of $\mathrm{Hg}$, millimeters of mercury; —, compound not detected at a concentration above the laboratory reporting level]

\begin{tabular}{|c|c|c|c|c|c|c|c|c|}
\hline $\begin{array}{c}\text { Date } \\
\text { (mm/dd/yyyy) }\end{array}$ & $\begin{array}{l}\text { Pressure } \\
(\mathrm{mm} \text { of } \mathrm{Hg})\end{array}$ & $\begin{array}{c}\text { Temperature } \\
\left({ }^{\circ} \mathrm{C}\right)\end{array}$ & $\begin{array}{l}\text { Dichloro- } \\
\text { difluoro- } \\
\text { methane } \\
\text { (CFC-12) }\end{array}$ & $\begin{array}{c}\text { Chloro- } \\
\text { methane }\end{array}$ & $\begin{array}{l}\text { Chloro- } \\
\text { ethene } \\
\text { (vinyl } \\
\text { chloride) }\end{array}$ & $\begin{array}{l}\text { Bromo- } \\
\text { methane }\end{array}$ & $\begin{array}{l}\text { Chloro- } \\
\text { ethane }\end{array}$ & $\begin{array}{c}\text { Bromo- } \\
\text { ethene } \\
\text { (vinyl } \\
\text { bromide) }\end{array}$ \\
\hline $10 / 22 / 2001$ & 753 & 17.8 & 0.89 & - & - & - & - & - \\
\hline $11 / 15 / 2001$ & 753 & 15.7 & 0.99 & - & - & - & - & - \\
\hline $11 / 27 / 2001$ & 755 & 11.2 & 0.28 & - & - & - & - & - \\
\hline $12 / 09 / 2001$ & 758 & 11.4 & 0.25 & - & - & - & - & - \\
\hline $12 / 21 / 2001$ & 753 & 10.6 & 0.77 & - & - & - & - & - \\
\hline $04 / 20 / 2002$ & 761 & 13.9 & 0.88 & - & - & - & - & - \\
\hline $02 / 14 / 2003$ & 753 & 14.9 & - & - & - & - & - & - \\
\hline $09 / 18 / 2003$ & 760 & 19.6 & 0.70 & - & - & - & - & - \\
\hline
\end{tabular}


Table 20B. Quality-control replicate results for volatile organic compounds with high breakthrough volumes from the Sweetwater Reservoir atmospheric site, San Diego County, California.

[The site identification number is 324141117001601 . Concentrations are given in parts per billion by volume (ppbv). Time is denoted in 24 -hour scale. ${ }^{\circ} \mathrm{C}$, degree Celsius; $\mathrm{mm}$ of $\mathrm{Hg}$, millimeters of mercury; E, estimated value; _, compound not detected at a concentration above the laboratory reporting level]

\begin{tabular}{|c|c|c|c|c|c|c|c|c|c|c|c|}
\hline $\begin{array}{c}\text { Date } \\
\text { (mm/dd/yyyy) }\end{array}$ & $\begin{array}{l}\text { Pressure } \\
(\mathrm{mm} \text { of } \mathrm{Hg})\end{array}$ & $\begin{array}{c}\text { Temper- } \\
\text { ature } \\
\left({ }^{\circ} \mathrm{C}\right)\end{array}$ & $\begin{array}{l}\text { Dibromo- } \\
\text { methane }\end{array}$ & $\begin{array}{l}\text { Bromo- } \\
\text { dichloro- } \\
\text { methane }\end{array}$ & $\begin{array}{c}\text { Carbon } \\
\text { tetra- } \\
\text { chloride }\end{array}$ & $\begin{array}{c}\text { 1,2- } \\
\text { Dichloro- } \\
\text { ethane }\end{array}$ & $\begin{array}{c}\text { Bromo- } \\
\text { form }\end{array}$ & $\begin{array}{c}\text { Dibromo- } \\
\text { chloro- } \\
\text { methane }\end{array}$ & $\begin{array}{l}\text { Chloro- } \\
\text { form }\end{array}$ & Toluene & Benzene \\
\hline $11 / 15 / 2001$ & 753 & 15.7 & - & - & E0.03 & - & - & - & E0.01 & 0.84 & 0.25 \\
\hline $11 / 27 / 2001$ & 755 & 11.2 & - & - & E0.14 & - & - & - & E0.04 & 1.09 & 0.45 \\
\hline $12 / 21 / 2001$ & 758 & 11.4 & E0.02 & - & - & - & - & - & - & 0.36 & 0.20 \\
\hline $01 / 14 / 2002$ & 753 & 10.6 & - & - & E0.23 & E0.02 & - & - & E0.04 & 1.51 & 1.04 \\
\hline $04 / 20 / 2002$ & 761 & 13.9 & - & - & E0.10 & - & - & - & E0.02 & 0.44 & - \\
\hline $02 / 14 / 2003$ & 753 & 14.9 & - & - & E0.09 & - & - & - & - & 0.32 & - \\
\hline 09/18/2003 & 760 & 19.6 & - & - & E0.01 & - & - & - & E0.01 & E0.07 & - \\
\hline $\begin{array}{c}\text { Date } \\
\text { (mm/dd/yyyy) }\end{array}$ & $\begin{array}{l}\text { Pressure } \\
(\mathrm{mm} \text { of } \mathrm{Hg})\end{array}$ & $\begin{array}{c}\text { Temper- } \\
\text { ature } \\
\left({ }^{\circ} \mathrm{C}\right)\end{array}$ & $\begin{array}{c}\text { 2-Propene- } \\
\text { nitrile } \\
\text { (Acrylo- } \\
\text { nitrile) }\end{array}$ & $\begin{array}{l}\text { Chloro- } \\
\text { benzene }\end{array}$ & $\begin{array}{c}\text { Ethyl- } \\
\text { benzene }\end{array}$ & $\begin{array}{l}\text { Hexa- } \\
\text { chloro- } \\
\text { ethane }\end{array}$ & $\begin{array}{c}\text { Methylene } \\
\text { chloride }\end{array}$ & $\begin{array}{l}\text { Tetra- } \\
\text { chloro- } \\
\text { ethene } \\
\text { (PCE) }\end{array}$ & $\begin{array}{c}\text { 1,1- } \\
\text { Dichloro- } \\
\text { ethane }\end{array}$ & $\begin{array}{c}\text { 1,1- } \\
\text { Dichloro- } \\
\text { ethene }\end{array}$ & $\begin{array}{c}\text { 1,1,1- } \\
\text { Trichloro- } \\
\text { ethane }\end{array}$ \\
\hline $11 / 15 / 2001$ & 753 & 15.7 & - & - & 0.08 & - & 0.12 & E0.02 & - & - & E0.01 \\
\hline $11 / 27 / 2001$ & 755 & 11.2 & - & - & 0.13 & - & 0.21 & E0.03 & - & - & E0.05 \\
\hline $12 / 21 / 2001$ & 758 & 11.4 & - & E0.01 & E0.02 & - & E0.08 & - & - & - & - \\
\hline $01 / 14 / 2002$ & 753 & 10.6 & - & - & 0.37 & - & 0.25 & E0.04 & - & - & 0.08 \\
\hline $04 / 20 / 2002$ & 761 & 13.9 & - & - & E0.06 & - & E0.08 & - & - & - & E0.03 \\
\hline $02 / 14 / 2003$ & 753 & 14.9 & - & - & E0.05 & - & E0.04 & E0.01 & - & - & E0.03 \\
\hline 09/18/2003 & 760 & 19.6 & - & E0.03 & E0.01 & - & E0.03 & E0.02 & - & - & - \\
\hline $\begin{array}{c}\text { Date } \\
\text { (mm/dd/yyyy) }\end{array}$ & $\begin{array}{c}\text { Pressure } \\
\text { (mm of } \mathrm{Hg})\end{array}$ & $\begin{array}{c}\text { Temper- } \\
\text { ature } \\
\left({ }^{\circ} \mathrm{C}\right)\end{array}$ & $\begin{array}{c}1,1,2- \\
\text { Trichloro- } \\
\text { ethane }\end{array}$ & $\begin{array}{l}\text { 1,1,2,2- } \\
\text { Tetra- } \\
\text { chloro- } \\
\text { ethane }\end{array}$ & $\begin{array}{c}1,2- \\
\text { Dichloro- } \\
\text { benzene }\end{array}$ & $\begin{array}{c}\text { 1,2- } \\
\text { Dichloro- } \\
\text { propane }\end{array}$ & $\begin{array}{c}\text { trans-1,2- } \\
\text { Dichloro- } \\
\text { ethene }\end{array}$ & $\begin{array}{c}\text { 1,2,4- } \\
\text { Trichloro- } \\
\text { benzene }\end{array}$ & $\begin{array}{c}\text { 1,3- } \\
\text { Dichloro- } \\
\text { benzene }\end{array}$ & $\begin{array}{c}\text { 1,4- } \\
\text { Dichloro- } \\
\text { benzene }\end{array}$ & $\begin{array}{l}\text { Naph- } \\
\text { thalene }\end{array}$ \\
\hline $11 / 15 / 2001$ & 753 & 15.7 & - & - & E0.01 & - & - & - & - & E0.01 & E0.01 \\
\hline $11 / 27 / 2001$ & 755 & 11.2 & - & - & E0.01 & - & - & - & - & E0.01 & E0.02 \\
\hline $12 / 21 / 2001$ & 758 & 11.4 & - & - & - & - & - & - & - & E0.01 & E0.01 \\
\hline $01 / 14 / 2002$ & 753 & 10.6 & - & - & - & - & - & - & - & E0.06 & 0.11 \\
\hline $04 / 20 / 2002$ & 761 & 13.9 & - & - & - & - & - & - & - & E0.01 & E0.02 \\
\hline $02 / 14 / 2003$ & 753 & 14.9 & - & - & - & - & - & - & - & E0.01 & E0.01 \\
\hline 09/18/2003 & 760 & 19.6 & - & - & - & - & - & - & - & - & E0.01 \\
\hline $\begin{array}{c}\text { Date } \\
\text { (mm/dd/yyyy) }\end{array}$ & $\begin{array}{l}\text { Pressure } \\
(\mathrm{mm} \text { of } \mathrm{Hg})\end{array}$ & $\begin{array}{c}\text { Temper- } \\
\text { ature } \\
\left({ }^{\circ} \mathrm{C}\right)\end{array}$ & $\begin{array}{l}\text { trans-1,3- } \\
\text { Dichloro- } \\
\text { propene }\end{array}$ & $\begin{array}{c}\text { cis-1,3- } \\
\text { Dichloro- } \\
\text { propene }\end{array}$ & $\begin{array}{c}\text { Trichloro- } \\
\text { ethene } \\
\text { (TCE) }\end{array}$ & $\begin{array}{c}\text { Hexa- } \\
\text { chloro- } \\
\text { butadiene }\end{array}$ & $\begin{array}{l}\text { Methyl } \\
\text { acrylate }\end{array}$ & $\begin{array}{c}\text { 1,2,3,4- } \\
\text { Tetra- } \\
\text { methyl- } \\
\text { benzene }\end{array}$ & $\begin{array}{c}\text { Ethyl } \\
\text { tert-butyl } \\
\text { ether } \\
\text { (ETBE) } \\
\end{array}$ & $\begin{array}{l}\text { tert-Amyl } \\
\text { methyl ether } \\
\text { (TAME) }\end{array}$ & $\begin{array}{l}\text { trans-1,4- } \\
\text { Dichloro- } \\
\text { 2-butene }\end{array}$ \\
\hline $11 / 15 / 2001$ & 753 & 15.7 & - & - & E0.02 & - & E0.01 & E0.01 & - & - & - \\
\hline $11 / 27 / 2001$ & 755 & 11.2 & - & - & E0.01 & - & E0.07 & E0.01 & - & - & - \\
\hline $12 / 21 / 2001$ & 758 & 11.4 & - & - & - & - & - & E0.01 & - & - & - \\
\hline 01/14/2002 & 753 & 10.6 & - & - & 0.08 & - & E0.13 & E0.02 & - & E0.03 & - \\
\hline 04/20/2002 & 761 & 13.9 & - & - & E0.01 & - & - & E0.01 & E0.01 & - & - \\
\hline $02 / 14 / 2003$ & 753 & 14.9 & - & - & E0.01 & - & - & E0.01 & - & - & - \\
\hline 09/18/2003 & 760 & 19.6 & - & - & - & - & - & E0.01 & E0.05 & - & - \\
\hline $\begin{array}{c}\text { Date } \\
\text { (mm/dd/yyyy) }\end{array}$ & $\begin{array}{l}\text { Pressure } \\
(\mathrm{mm} \text { of } \mathrm{Hg})\end{array}$ & $\begin{array}{c}\text { Temper- } \\
\text { ature } \\
\left({ }^{\circ} \mathrm{C}\right)\end{array}$ & $\begin{array}{l}\text { Ethyl- } \\
\text { meth- } \\
\text { acrylate }\end{array}$ & $\begin{array}{l}\text { Carbon } \\
\text { disulfide }\end{array}$ & $\begin{array}{c}\text { cis-1,2- } \\
\text { Dichloro- } \\
\text { ethene }\end{array}$ & $\begin{array}{c}\text { 2-Hexa- } \\
\text { none } \\
\text { (MBK) }\end{array}$ & $\begin{array}{l}\text { Ethenyl- } \\
\text { benzene } \\
\text { (Styrene) }\end{array}$ & o-Xylene & $\begin{array}{c}\text { 1,1- } \\
\text { Dichloro- } \\
\text { propene }\end{array}$ & $\begin{array}{c}\text { 2,2- } \\
\text { Dichloro- } \\
\text { propane }\end{array}$ & $\begin{array}{c}\text { 1,3- } \\
\text { Dichloro- } \\
\text { propane }\end{array}$ \\
\hline $11 / 15 / 2001$ & 753 & 15.7 & - & E0.02 & - & - & - & 0.09 & - & - & - \\
\hline $11 / 27 / 2001$ & 755 & 11.2 & - & - & - & - & E0.02 & 0.16 & - & - & - \\
\hline $12 / 21 / 2001$ & 758 & 11.4 & - & - & - & E0.01 & E0.01 & E0.02 & - & - & - \\
\hline $01 / 14 / 2002$ & 753 & 10.6 & - & E0.02 & - & E0.03 & 0.09 & 0.42 & - & - & - \\
\hline $04 / 20 / 2002$ & 761 & 13.9 & - & - & - & E0.01 & - & E0.06 & - & - & - \\
\hline $02 / 14 / 2003$ & 753 & 14.9 & - & - & - & E0.01 & E0.01 & E0.07 & - & - & - \\
\hline 09/18/2003 & 760 & 19.6 & - & E0.04 & - & E0.03 & E0.03 & E0.01 & - & - & - \\
\hline
\end{tabular}


Table 20B. Quality-control replicate results for volatile organic compounds with high breakthrough volumes from the Sweetwater Reservoir atmospheric site, San Diego County, California-Continued

[The site identification number is 324141117001601 . Concentrations are given in parts per billion by volume (ppbv). Time is denoted in 24 -hour scale. ${ }^{\circ} \mathrm{C}$, degree Celsius; $\mathrm{mm}$ of $\mathrm{Hg}$, millimeters of mercury; E, estimated value; _, compound not detected at a concentration above the laboratory reporting level]

\begin{tabular}{|c|c|c|c|c|c|c|c|c|c|c|c|}
\hline $\begin{array}{c}\text { Date } \\
\text { (mm/dd/yyyy) }\end{array}$ & $\begin{array}{l}\text { Pressure } \\
(\mathbf{m m} \text { of } \mathrm{Hg})\end{array}$ & $\begin{array}{c}\text { Temper- } \\
\text { ature } \\
\left({ }^{\circ} \mathrm{C}\right)\end{array}$ & $\begin{array}{c}2- \\
\text { Ethyl- } \\
\text { toluene }\end{array}$ & $\begin{array}{c}1,2,3- \\
\text { Trimethyl- } \\
\text { benzene }\end{array}$ & $\begin{array}{c}1,2,4- \\
\text { Trimethyl- } \\
\text { benzene }\end{array}$ & $\begin{array}{c}\text { Isopropyl- } \\
\text { benzene } \\
\text { (Cumene) }\end{array}$ & $\begin{array}{l}n \text {-propyl- } \\
\text { benzene }\end{array}$ & $\begin{array}{c}1,3,5- \\
\text { Trimethyl- } \\
\text { benzene }\end{array}$ & $\begin{array}{l}\text { 2-Chloro- } \\
\text { toluene }\end{array}$ & $\begin{array}{l}\text { 4-Chloro- } \\
\text { toluene }\end{array}$ & $\begin{array}{l}\text { Bromo- } \\
\text { chloro- } \\
\text { methane }\end{array}$ \\
\hline $11 / 15 / 2001$ & 753 & 15.7 & E0.02 & E0.01 & 0.08 & E0.01 & E0.02 & E0.02 & - & - & - \\
\hline $12 / 21 / 2001$ & 758 & 11.4 & E0.01 & E0.01 & E0.03 & E0.01 & E0.01 & E0.01 & - & - & - \\
\hline 01/14/2002 & 753 & 10.6 & 0.09 & 0.08 & 0.32 & E0.02 & E0.07 & 0.08 & - & - & - \\
\hline $04 / 20 / 2002$ & 761 & 13.9 & E0.01 & E0.01 & E0.05 & E0.01 & E0.01 & E0.01 & - & - & - \\
\hline $\begin{array}{c}\text { Date } \\
\text { (mm/dd/yyyy) }\end{array}$ & $\begin{array}{l}\text { Pressure } \\
(\mathrm{mm} \text { of } \mathrm{Hg})\end{array}$ & $\begin{array}{c}\text { Temper- } \\
\text { ature } \\
\left({ }^{\circ} \mathrm{C}\right)\end{array}$ & $\begin{array}{l}n \text {-Butyl- } \\
\text { benzene }\end{array}$ & $\begin{array}{c}\text { sec- } \\
\text { Butyl- } \\
\text { benzene }\end{array}$ & $\begin{array}{c}\text { tert- } \\
\text { Butyl } \\
\text { benzene }\end{array}$ & $\begin{array}{c}\text { 1- } \\
\text { Isopropyl- } \\
\text { 4-methyl } \\
\text { benzene }\end{array}$ & $\begin{array}{c}\text { 1,2,3- } \\
\text { Trichloro- } \\
\text { propene }\end{array}$ & $\begin{array}{l}1,1,1,2- \\
\text { Tetra- } \\
\text { chloro- } \\
\text { ethane }\end{array}$ & $\begin{array}{c}\text { 1,2,3- } \\
\text { Trichloro- } \\
\text { benzene }\end{array}$ & $\begin{array}{c}1,2- \\
\text { Dibromo- } \\
\text { ethane }\end{array}$ & $\begin{array}{c}\text { Methyl } \\
\text { tert-butyl } \\
\text { ether } \\
\text { (MTBE) }\end{array}$ \\
\hline $11 / 15 / 2001$ & 753 & 15.7 & E0.01 & E0.01 & - & E0.02 & - & - & - & - & 0.76 \\
\hline $02 / 14 / 2003$ & 753 & 14.9 & E0.01 & E0.01 & - & E0.02 & - & - & - & - & 0.19 \\
\hline 09/18/2003 & 760 & 19.6 & E0.01 & - & - & E0.01 & - & - & - & - & E0.02 \\
\hline $\begin{array}{c}\text { Date } \\
\text { (mm/dd/yyyy) }\end{array}$ & $\begin{array}{l}\text { Pressure } \\
(\mathbf{m m} \text { of } \mathrm{Hg})\end{array}$ & $\begin{array}{c}\text { Temper- } \\
\text { ature } \\
\left({ }^{\circ} \mathrm{C}\right)\end{array}$ & $\begin{array}{l}\text { 3-Chloro- } \\
\text { 1-propene }\end{array}$ & $\begin{array}{l}\text { 4-Methyl-2- } \\
\text { pentanone } \\
\text { (MIBK) }\end{array}$ & Acetone & $\begin{array}{l}\text { Bromo- } \\
\text { benzene }\end{array}$ & $\begin{array}{l}\text { Diethyl } \\
\text { ether }\end{array}$ & $\begin{array}{l}\text { Diiso- } \\
\text { propyl } \\
\text { ether } \\
\text { (DIPE) }\end{array}$ & $\begin{array}{l}\text { Methyl- } \\
\text { acrylo- } \\
\text { nitrile }\end{array}$ & $\begin{array}{c}\text { 2-Butanone } \\
\text { (Methyl ethyl } \\
\text { ketone) }\end{array}$ & $\begin{array}{c}\text { Methyl- } \\
\text { meth- } \\
\text { acrylate }\end{array}$ \\
\hline $11 / 15 / 2001$ & 753 & 15.7 & - & - & - & - & - & - & - & 0.18 & - \\
\hline $11 / 27 / 2001$ & 755 & 11.2 & - & - & 0.95 & - & - & - & - & 0.24 & - \\
\hline $12 / 21 / 2001$ & 758 & 11.4 & - & E0.01 & 0.58 & - & - & - & - & E0.12 & - \\
\hline 01/14/2002 & 753 & 10.6 & - & E0.06 & 3.91 & - & - & - & - & 1.21 & - \\
\hline 04/20/2002 & 761 & 13.9 & - & E0.01 & 1.25 & - & E0.01 & - & - & 0.20 & - \\
\hline $11 / 27 / 2001$ & 755 & 11.2 & - & - & 0.55 & E0.01 & E0.01 & - & - & E0.04 & \\
\hline $12 / 21 / 2001$ & 758 & 11.4 & - & - & E0.05 & E0.01 & E0.01 & - & - & E0.06 & \\
\hline 01/14/2002 & 753 & 10.6 & E0.06 & - & 1.09 & E0.05 & E0.03 & - & E0.11 & 0.25 & \\
\hline 04/20/2002 & 761 & 13.9 & - & - & 0.18 & E0.01 & E0.01 & - & E0.02 & E0.12 & \\
\hline $02 / 14 / 2003$ & 753 & 14.9 & - & - & 0.19 & E0.01 & E0.01 & - & E0.01 & E0.05 & \\
\hline 09/18/2003 & 760 & 19.6 & - & - & E0.03 & E0.01 & E0.01 & - & E0.09 & E0.11 & \\
\hline
\end{tabular}


Table 21A. Quality-control results for volatile organic compounds with low breakthrough volumes in laboratory spikes for air samples collected at the Sweetwater Reservoir atmospheric site, San Diego County, California.

[Values are given in percent recovery. Sample volume for quality assurance spike is 1.5 liters. Samples were processed at 20.0 degrees Celsius and 760 millimeters of mercury. Dates are in month/day/year format. NA, not analyzed]

\begin{tabular}{|c|c|c|c|c|c|c|c|}
\hline $\begin{array}{c}\text { Sampling } \\
\text { date }\end{array}$ & $\begin{array}{c}\text { Analyzed } \\
\text { date }\end{array}$ & $\begin{array}{l}\text { Dichloro- } \\
\text { difluoro- } \\
\text { methane } \\
\text { (CFC-12) }\end{array}$ & $\begin{array}{l}\text { Chloro- } \\
\text { methane }\end{array}$ & $\begin{array}{l}\text { Chloro- } \\
\text { ethene } \\
\text { (vinyl } \\
\text { chloride) }\end{array}$ & $\begin{array}{l}\text { Bromo- } \\
\text { methane }\end{array}$ & $\begin{array}{l}\text { Chloro- } \\
\text { ethane }\end{array}$ & $\begin{array}{c}\text { Bromo- } \\
\text { ethene } \\
\text { (vinyl } \\
\text { bromide) }\end{array}$ \\
\hline $12 / 09 / 2001$ & $12 / 28 / 2001$ & 122 & 128 & 93.2 & 53.2 & 82.3 & 114 \\
\hline 06/07/2002 & $06 / 28 / 2002$ & 127 & 93.8 & 94.7 & NA & 112 & 108 \\
\hline $07 / 25 / 2002$ & 08/08/2002 & 91.9 & 73.8 & 70.2 & NA & 79.7 & 71.6 \\
\hline $02 / 14 / 2003$ & 03/07/2003 & 106 & 107 & 94.0 & NA & 61.4 & 96.6 \\
\hline
\end{tabular}


Table 21B. Quality-control results for volatile organic compounds with low breakthrough volumes in travel blanks for air samples collected at the Sweetwater Reservoir atmospheric site, San Diego County, California.

[Concentrations are given in parts per billion by volume (ppbv). Sample volume for quality assurance travel blank is 1.5 liters. Dates are in month/day/year format. Pairs of samples analyzed on the same date had been frozen and stored at the laboratory before analysis. Samples were processed at 20.0 degrees Celsius and 760 millimeters of mercury. $\mathrm{mm}$ of $\mathrm{Hg}$, millimeters of mercury; - , compound not detected at a concentration above laboratory reporting level; ${ }^{\circ} \mathrm{C}$, degree Celsius; E, estimated value; NA, not analyzed]

\begin{tabular}{|c|c|c|c|c|c|c|c|c|c|}
\hline $\begin{array}{l}\text { Sampling } \\
\text { date }\end{array}$ & $\begin{array}{l}\text { Analyzed } \\
\text { date }\end{array}$ & $\begin{array}{l}\text { Pressure } \\
(\mathrm{mm} \text { of } \mathrm{Hg})\end{array}$ & $\begin{array}{c}\text { Temper- } \\
\text { ature } \\
\left({ }^{\circ} \mathrm{C}\right)\end{array}$ & $\begin{array}{l}\text { Dichloro- } \\
\text { difluoro- } \\
\text { methane } \\
\text { (CFC-12) }\end{array}$ & $\begin{array}{l}\text { Chloro- } \\
\text { methane }\end{array}$ & $\begin{array}{l}\text { Chloro- } \\
\text { ethene } \\
\text { (vinyl } \\
\text { chloride) }\end{array}$ & $\begin{array}{l}\text { Bromo- } \\
\text { methane }\end{array}$ & $\begin{array}{l}\text { Chloro- } \\
\text { ethane }\end{array}$ & $\begin{array}{c}\text { Bromo- } \\
\text { ethene } \\
\text { (vinyl } \\
\text { bromide) }\end{array}$ \\
\hline $10 / 10 / 2001$ & $10 / 26 / 2001$ & 754 & 18.8 & E0.018 & E0.070 & - & - & - & - \\
\hline $10 / 22 / 2001$ & $10 / 26 / 2001$ & 753 & 17.8 & E0.010 & - & - & - & - & - \\
\hline $11 / 15 / 2001$ & $11 / 30 / 2001$ & 753 & 15.7 & E0.023 & - & - & - & - & - \\
\hline $12 / 21 / 2001$ & $12 / 28 / 2001$ & 758 & 11.4 & E0.016 & - & - & - & - & - \\
\hline 01/03/2002 & $01 / 22 / 2002$ & 757 & 11.9 & NA & NA & NA & NA & NA & NA \\
\hline $01 / 14 / 2002$ & $01 / 22 / 2002$ & 753 & 10.6 & NA & NA & NA & NA & NA & NA \\
\hline $01 / 28 / 2002$ & $02 / 15 / 2002$ & 754 & 10.6 & 0.421 & - & - & 0.924 & - & - \\
\hline $02 / 07 / 2002$ & $02 / 15 / 2002$ & 761 & 10.2 & E0.008 & - & - & E0.465 & - & E0.002 \\
\hline $02 / 19 / 2002$ & 03/08/2002 & 760 & 10.9 & E0.025 & - & - & E0.413 & - & - \\
\hline $05 / 14 / 2002$ & $05 / 31 / 2002$ & 751 & 17.0 & - & 1.82 & - & - & - & - \\
\hline $05 / 26 / 2002$ & $05 / 31 / 2002$ & 753 & 13.4 & - & 1.46 & - & - & - & - \\
\hline 06/07/2002 & $06 / 28 / 2002$ & 750 & 16.2 & E0.008 & - & - & - & - & - \\
\hline 06/19/2002 & $06 / 28 / 2002$ & 749 & 16.3 & E0.015 & - & - & - & - & - \\
\hline 07/01/2002 & 07/19/2002 & 753 & 16.9 & E0.022 & - & - & - & E0.101 & - \\
\hline $07 / 13 / 2002$ & 07/19/2002 & 751 & 18.1 & E0.022 & - & - & - & - & - \\
\hline $07 / 25 / 2002$ & 08/08/2002 & 754 & 18.7 & E0.009 & - & - & - & - & - \\
\hline 08/06/2002 & 08/08/2002 & 753 & 17.2 & E0.022 & - & - & - & - & - \\
\hline 08/18/2002 & 09/06/2002 & 752 & 18.5 & E0.006 & - & - & - & - & - \\
\hline 08/30/2002 & 09/06/2002 & 751 & 20.8 & E0.019 & - & - & - & - & - \\
\hline 09/11/2002 & 09/27/2002 & 750 & 21.8 & E0.013 & - & - & - & - & - \\
\hline $09 / 23 / 2002$ & 09/27/2002 & 749 & 22.7 & E0.010 & - & - & - & - & - \\
\hline $02 / 04 / 2003$ & 02/07/2003 & 754 & 13.8 & E0.008 & - & - & - & - & - \\
\hline $02 / 14 / 2003$ & 03/07/2003 & 753 & 14.9 & E0.024 & - & - & - & - & - \\
\hline $02 / 14 / 2003$ & 03/07/2003 & 753 & 14.9 & E0.255 & - & 0.126 & - & 0.044 & - \\
\hline $02 / 26 / 2003$ & 03/07/2003 & 752 & 12.6 & E0.013 & - & - & - & - & - \\
\hline 03/10/2003 & 03/28/2003 & 753 & 15.0 & E0.006 & - & - & - & - & - \\
\hline $03 / 22 / 2003$ & $03 / 28 / 2003$ & 756 & 15.4 & E0.010 & - & - & - & - & - \\
\hline 04/03/2003 & $04 / 18 / 2003$ & 757 & 12.4 & E0.019 & - & - & - & - & - \\
\hline $04 / 15 / 2003$ & $04 / 18 / 2003$ & 757 & 12.6 & E0.024 & - & - & - & - & - \\
\hline 05/09/2003 & $05 / 15 / 2003$ & 762 & 13.8 & E0.041 & - & - & - & - & - \\
\hline $05 / 21 / 2003$ & 06/06/2003 & 758 & 20.2 & E0.036 & - & - & - & - & - \\
\hline $06 / 02 / 2003$ & 06/06/2003 & 758 & 16.4 & E0.034 & - & - & - & - & - \\
\hline $06 / 14 / 2003$ & $06 / 30 / 2003$ & 759 & 19.1 & E0.010 & - & - & - & - & - \\
\hline $06 / 26 / 2003$ & 06/30/2003 & 759 & 19.2 & E0.014 & - & - & - & - & - \\
\hline
\end{tabular}


Table 21B. Quality-control results for volatile organic compounds with low breakthrough volumes in travel blanks for air samples collected at the Sweetwater Reservoir Atmospheric site, San Diego County, California._-Continued

[Concentrations are given in parts per billion by volume (ppbv). Sample volume for quality assurance travel blank is 1.5 liters. Dates are in month/day/year format. Pairs of samples analyzed on the same date had been frozen and stored at the laboratory before analysis. Samples were processed at 20.0 degrees Celsius and 760 millimeters of mercury. $\mathrm{mm}$ of $\mathrm{Hg}$, millimeters of mercury; - , compound not detected at a concentration above laboratory reporting level; ${ }^{\circ} \mathrm{C}$, degree Celsius; E, estimated value; NA, not analyzed]

\begin{tabular}{|c|c|c|c|c|c|c|c|c|c|}
\hline $\begin{array}{l}\text { Sampling } \\
\text { date }\end{array}$ & $\begin{array}{c}\text { Analyzed } \\
\text { date }\end{array}$ & $\begin{array}{l}\text { Pressure } \\
(\mathrm{mm} \text { of } \mathrm{Hg})\end{array}$ & $\begin{array}{c}\text { Temper- } \\
\text { ature } \\
\left({ }^{\circ} \mathrm{C}\right)\end{array}$ & $\begin{array}{l}\text { Dichloro- } \\
\text { difluoro- } \\
\text { methane } \\
\text { (CFC-12) }\end{array}$ & $\begin{array}{l}\text { Chloro- } \\
\text { methane }\end{array}$ & $\begin{array}{l}\text { Chloro- } \\
\text { ethene } \\
\text { (vinyl } \\
\text { chloride) }\end{array}$ & $\begin{array}{l}\text { Bromo- } \\
\text { methane }\end{array}$ & $\begin{array}{l}\text { Chloro- } \\
\text { ethane }\end{array}$ & $\begin{array}{c}\text { Bromo- } \\
\text { ethene } \\
\text { (vinyl } \\
\text { bromide) }\end{array}$ \\
\hline $07 / 09 / 2003$ & $07 / 25 / 2003$ & 758 & 18.8 & E0.040 & - & - & - & - & - \\
\hline $07 / 20 / 2003$ & $07 / 25 / 2003$ & 761 & 22.9 & E0.017 & - & - & - & - & - \\
\hline 08/01/2003 & $08 / 15 / 2003$ & 762 & 23.0 & E0.047 & - & - & - & - & - \\
\hline $09 / 06 / 2003$ & $09 / 29 / 2003$ & 759 & 24.4 & E0.018 & - & - & - & - & - \\
\hline 09/18/2003 & $09 / 29 / 2003$ & 757 & 19.5 & E0.013 & - & - & - & - & - \\
\hline $09 / 18 / 2003$ & $10 / 16 / 2003$ & 760 & 19.6 & E0.015 & - & - & - & - & - \\
\hline
\end{tabular}


Table 21C. Quality-control results for volatile organic compounds with low breakthrough volumes in lot blanks processed with air samples collected at the Sweetwater Reservoir atmospheric site, San Diego County, California.

[Concentrations are given in parts per billion by volume (ppbv). Sample volume for the quality assurance lot blank is 1.5 liters. Dates are in month/day/year format. Pairs of samples analyzed on the same date had been frozen and stored at the laboratory before analysis. Samples were processed at 20.0 degrees Celsius and 760 millimeters of mercury. —, compound not detected at a concentration above laboratory reporting level; E, estimated value; NA, not analyzed]

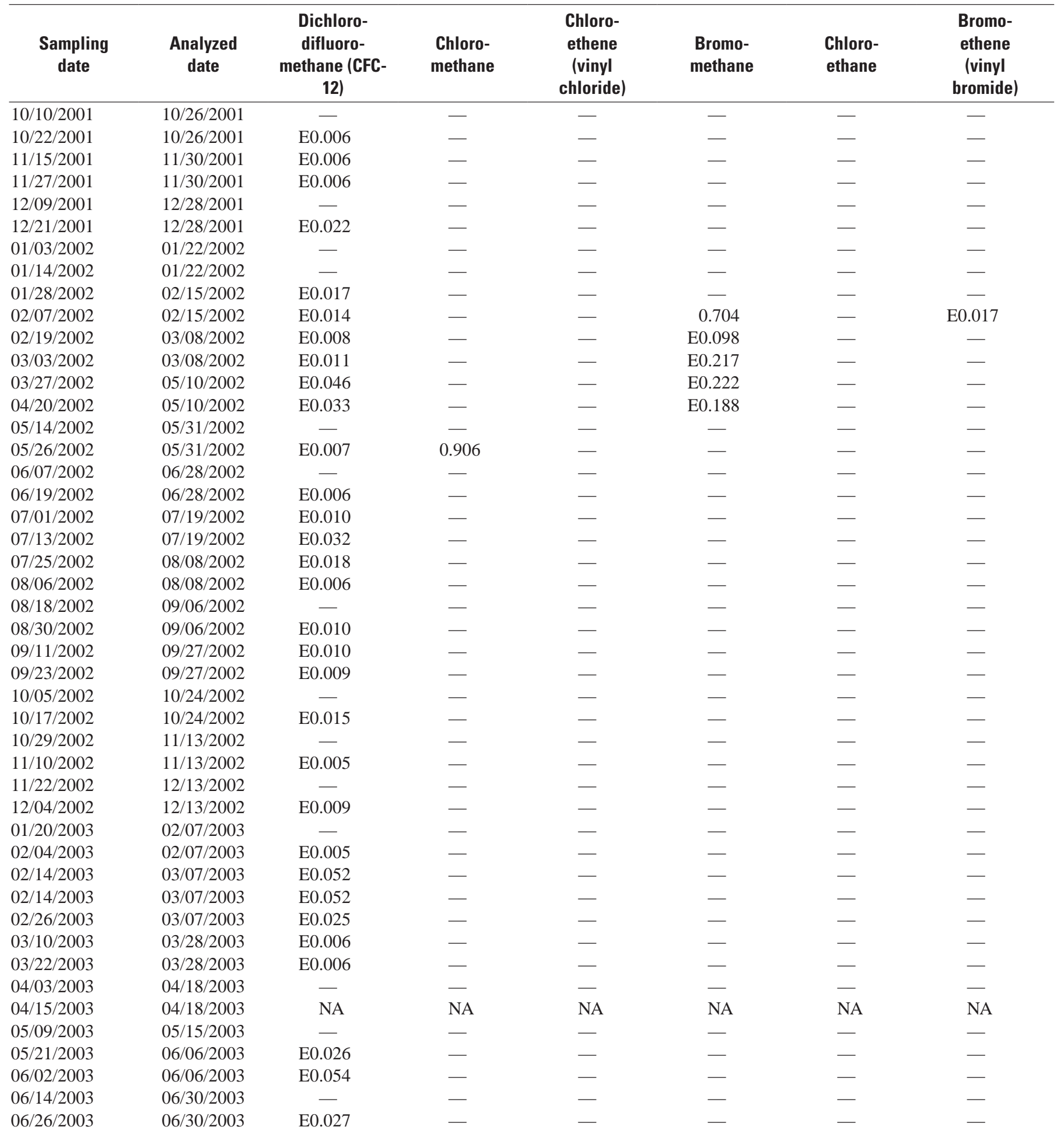


Table 21C. Quality-control results for volatile organic compounds with low breakthrough volumes in lot blanks processed with air samples collected at the Sweetwater Reservoir atmospheric site, San Diego County, California.—Continued

[Concentrations are given in parts per billion by volume (ppbv). Sample volume for the quality assurance lot blank is 1.5 liters. Dates are in month/day/year format. Pairs of samples analyzed on the same date had been frozen and stored at the laboratory before analysis. Samples were processed at 20.0 degrees Celsius and 760 millimeters of mercury. —, compound not detected at a concentration above laboratory reporting level; E, estimated value; NA, not analyzed]

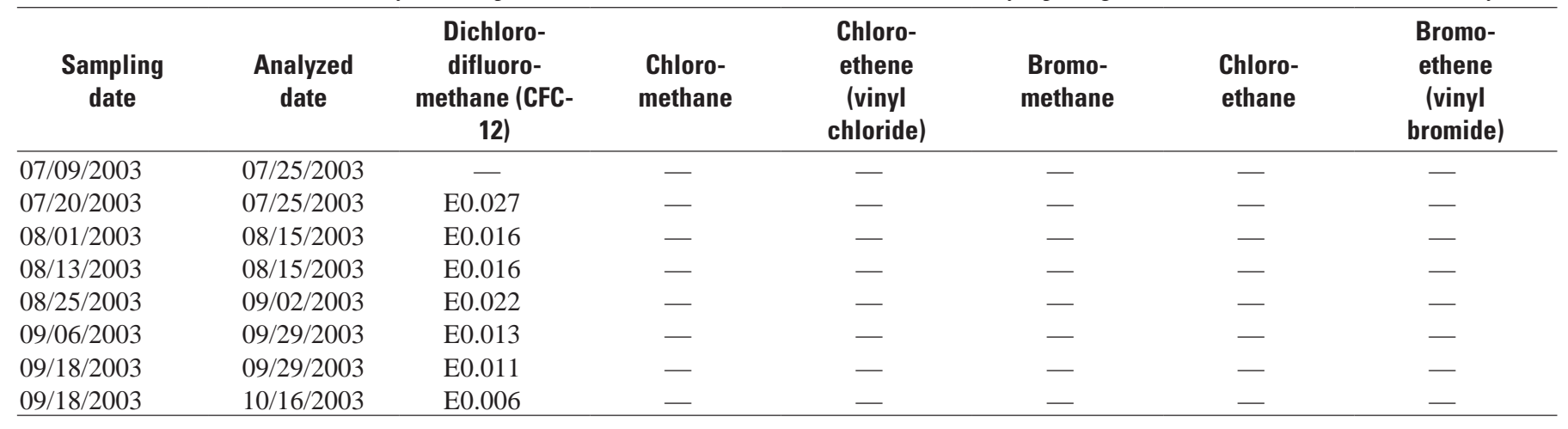


Table 21D. Quality-control results for volatile organic compounds with low breakthrough volumes in laboratory blanks for air samples collected at the Sweetwater Reservoir atmospheric site, San Diego County, California.

[Concentrations are given in parts per billion by volume (ppbv). Sample volume for quality assurance laboratory blank is 1.5 liters. Dates are in month/day/year format. Pairs of samples analyzed on the same date had been frozen and stored at the laboratory before analysis. Samples were processed at 20.0 degrees Celsius and 760 millimeters of mercury. —, compound not detected at a concentration above laboratory reporting level; E, estimated value; NA, not analyzed]

\begin{tabular}{|c|c|c|c|c|c|c|c|}
\hline $\begin{array}{l}\text { Sampling } \\
\text { date }\end{array}$ & $\begin{array}{l}\text { Analyzed } \\
\text { date }\end{array}$ & $\begin{array}{c}\text { Dichloro- } \\
\text { difluoro- } \\
\text { methane (CFC- } \\
\text { 12) }\end{array}$ & $\begin{array}{l}\text { Chloro- } \\
\text { methane }\end{array}$ & $\begin{array}{c}\text { Chloroethene } \\
\text { (vinyl } \\
\text { chloride) }\end{array}$ & $\begin{array}{l}\text { Bromo- } \\
\text { methane }\end{array}$ & $\begin{array}{l}\text { Chloro- } \\
\text { ethane }\end{array}$ & $\begin{array}{c}\text { Bromo- } \\
\text { ethene } \\
\text { (vinyl } \\
\text { bromide) }\end{array}$ \\
\hline 10/10/2001 & $10 / 26 / 2001$ & E0.010 & - & - & - & - & - \\
\hline $10 / 22 / 2001$ & $10 / 26 / 2001$ & E0.008 & - & - & - & - & - \\
\hline $11 / 15 / 2001$ & $11 / 30 / 2001$ & E0.013 & - & - & - & - & - \\
\hline $11 / 27 / 2001$ & $11 / 30 / 2001$ & E0.010 & - & - & - & - & - \\
\hline $12 / 09 / 2001$ & $12 / 28 / 2001$ & E0.031 & - & - & - & - & - \\
\hline $12 / 21 / 2001$ & $12 / 28 / 2001$ & E0.010 & - & - & - & - & - \\
\hline 01/03/2002 & $01 / 22 / 2002$ & NA & NA & NA & NA & NA & NA \\
\hline 01/14/2002 & $01 / 22 / 2002$ & NA & NA & NA & NA & NA & NA \\
\hline $01 / 28 / 2002$ & $02 / 15 / 2002$ & E0.008 & - & - & 0.600 & - & - \\
\hline $02 / 07 / 2002$ & $02 / 15 / 2002$ & E0.005 & - & - & E0.427 & - & E0.081 \\
\hline 02/19/2002 & 03/08/2002 & E0.016 & - & - & E0.400 & - & - \\
\hline 03/03/2002 & 03/08/2002 & E0.011 & - & - & E0.250 & - & - \\
\hline 03/27/2002 & $05 / 10 / 2002$ & E0.088 & - & - & 0.849 & - & - \\
\hline $04 / 20 / 2002$ & $05 / 10 / 2002$ & E0.034 & - & - & E0.276 & - & - \\
\hline 05/14/2002 & $05 / 31 / 2002$ & E0.007 & 2.03 & - & - & - & - \\
\hline 05/26/2002 & $05 / 31 / 2002$ & E0.008 & 1.74 & - & - & - & - \\
\hline $06 / 07 / 2002$ & $06 / 28 / 2002$ & E0.011 & - & - & - & - & - \\
\hline 06/19/2002 & $06 / 28 / 2002$ & E0.009 & - & - & - & - & - \\
\hline 07/01/2002 & 07/19/2002 & E0.016 & - & - & - & - & - \\
\hline 07/13/2002 & 07/19/2002 & E0.012 & - & - & - & - & - \\
\hline $07 / 25 / 2002$ & 08/08/2002 & E0.010 & - & - & - & - & - \\
\hline 08/06/2002 & 08/08/2002 & E0.007 & - & - & - & - & - \\
\hline 08/18/2002 & 09/06/2002 & E0.010 & - & - & - & - & - \\
\hline 08/30/2002 & 09/06/2002 & E0.008 & - & - & - & - & - \\
\hline 09/11/2002 & 09/27/2002 & E0.010 & - & - & - & - & - \\
\hline 09/23/2002 & 09/27/2002 & E0.008 & - & - & - & - & - \\
\hline $10 / 05 / 2002$ & $10 / 24 / 2002$ & E0.010 & - & - & - & - & - \\
\hline $10 / 17 / 2002$ & $10 / 24 / 2002$ & E0.024 & - & - & - & - & - \\
\hline $10 / 29 / 2002$ & $11 / 13 / 2002$ & E0.010 & - & - & - & - & - \\
\hline $11 / 10 / 2002$ & $11 / 13 / 2002$ & E0.010 & - & - & - & - & - \\
\hline $11 / 22 / 2002$ & $12 / 13 / 2002$ & E0.009 & - & - & - & - & - \\
\hline $12 / 04 / 2002$ & $12 / 13 / 2002$ & E0.007 & - & - & - & - & - \\
\hline $01 / 20 / 2003$ & 02/07/2003 & E0.010 & - & - & - & - & - \\
\hline $02 / 04 / 2003$ & 02/07/2003 & E0.010 & - & - & - & - & - \\
\hline $02 / 14 / 2003$ & $03 / 07 / 2003$ & E0.012 & - & - & - & - & - \\
\hline $02 / 14 / 2003$ & 03/07/2003 & E0.012 & - & - & - & - & - \\
\hline $02 / 26 / 2003$ & 03/07/2003 & E0.013 & - & - & - & - & - \\
\hline 03/10/2003 & 03/28/2003 & E0.007 & - & - & - & - & - \\
\hline $03 / 22 / 2003$ & 03/28/2003 & - & - & - & - & - & - \\
\hline $04 / 03 / 2003$ & $04 / 18 / 2003$ & E0.007 & - & - & - & - & - \\
\hline $04 / 15 / 2003$ & $04 / 18 / 2003$ & NA & NA & NA & - & - & NA \\
\hline 05/09/2003 & $05 / 15 / 2003$ & - & - & - & - & - & - \\
\hline $05 / 21 / 2003$ & 06/06/2003 & E0.040 & - & - & - & - & - \\
\hline $06 / 02 / 2003$ & 06/06/2003 & E0.046 & - & - & - & - & - \\
\hline $06 / 14 / 2003$ & $06 / 30 / 2003$ & - & - & - & - & - & - \\
\hline $06 / 26 / 2003$ & 06/30/2003 & E0.009 & - & - & - & - & - \\
\hline 07/09/2003 & $07 / 25 / 2003$ & E0.072 & - & - & - & - & - \\
\hline
\end{tabular}


Table 21D. Quality-control results for volatile organic compounds with low breakthrough volumes in laboratory blanks for air samples collected at the Sweetwater Reservoir atmospheric site, San Diego County, California.—Continued

[Concentrations are given in parts per billion by volume (ppbv). Sample volume for quality assurance laboratory blank is 1.5 liters. Dates are in month/day/ year format. Pairs of samples analyzed on the same date had been frozen and stored at the laboratory before analysis. Samples were processed at 20.0 degrees Celsius and 760 millimeters of mercury. —, compound not detected at a concentration above laboratory reporting level; E, estimated value; NA, not analyzed]

\begin{tabular}{|c|c|c|c|c|c|c|c|}
\hline $\begin{array}{l}\text { Sampling } \\
\text { date }\end{array}$ & $\begin{array}{c}\text { Analyzed } \\
\text { date }\end{array}$ & $\begin{array}{c}\text { Dichloro- } \\
\text { difluoro- } \\
\text { methane (CFC- } \\
\text { 12) }\end{array}$ & $\begin{array}{l}\text { Chloro- } \\
\text { methane }\end{array}$ & $\begin{array}{c}\text { Chloroethene } \\
\text { (vinyl } \\
\text { chloride) }\end{array}$ & $\begin{array}{l}\text { Bromo- } \\
\text { methane }\end{array}$ & $\begin{array}{l}\text { Chloro- } \\
\text { ethane }\end{array}$ & $\begin{array}{c}\text { Bromo- } \\
\text { ethene } \\
\text { (vinyl } \\
\text { bromide) }\end{array}$ \\
\hline 08/01/2003 & $08 / 15 / 2003$ & E0.010 & - & - & - & - & - \\
\hline 08/13/2003 & 08/15/2003 & E0.009 & - & - & - & - & - \\
\hline $08 / 25 / 2003$ & $09 / 2 / 2003$ & E0.010 & - & - & - & - & - \\
\hline 09/18/2003 & 09/29/2003 & E0.011 & - & - & - & - & - \\
\hline 09/18/2003 & $10 / 16 / 2003$ & E0.010 & - & - & - & - & - \\
\hline
\end{tabular}


Table 22A. Quality-control results for volatile organic compounds with high breakthrough volumes in laboratory spikes for air samples collected at the Sweetwater Reservoir atmospheric site, San Diego County, California.

[Values are given in percent recovery. Sample volume for quality assurance spike is 5 liters. Samples were processed at 20.0 degrees Celsius and 760 millimeters of mercury. Dates are in month/day/year format]

\begin{tabular}{cccccccc}
\hline $\begin{array}{c}\text { Sampling } \\
\text { date }\end{array}$ & $\begin{array}{c}\text { Analyzed } \\
\text { date }\end{array}$ & $\begin{array}{c}\text { Dibromo- } \\
\text { methane }\end{array}$ & $\begin{array}{c}\text { Bromo- } \\
\text { dichloro- } \\
\text { methane }\end{array}$ & $\begin{array}{c}\text { Carbon } \\
\text { tetra- } \\
\text { chloride }\end{array}$ & $\begin{array}{c}\text { 1,2-Dichloro- } \\
\text { ethane }\end{array}$ & $\begin{array}{c}\text { Bromo- } \\
\text { form }\end{array}$ & $\begin{array}{c}\text { Dibromo- } \\
\text { chloro- } \\
\text { methane }\end{array}$ \\
\hline $12 / 09 / 2001$ & $12 / 28 / 2001$ & 78.3 & 86.6 & 129 & 75.3 & 103 & 99.1 \\
$06 / 07 / 2002$ & $06 / 28 / 2002$ & 70.7 & 99.6 & 109 & 128 & 97.2 & 104 \\
$07 / 25 / 2002$ & $08 / 08 / 2002$ & 125 & 93.9 & 95.4 & 75.3 & 75.2 & 105 \\
$10 / 05 / 2002$ & $10 / 24 / 2002$ & 66.5 & 108 & 94.3 & 96.6 & 99.3 & 107 \\
$02 / 14 / 2003$ & $03 / 07 / 2003$ & 71.6 & 84.2 & 71.7 & 70.6 & 84.7 & 93.5 \\
Chloroform
\end{tabular}

\begin{tabular}{|c|c|c|c|c|c|c|c|c|}
\hline $\begin{array}{l}\text { Sampling } \\
\text { date }\end{array}$ & $\begin{array}{l}\text { Analyzed } \\
\text { date }\end{array}$ & Toluene & Benzene & $\begin{array}{c}\text { 2-Propene- } \\
\text { nitrile } \\
\text { (Acrylo- } \\
\text { nitrile) } \\
\end{array}$ & $\begin{array}{l}\text { Chloro- } \\
\text { benzene }\end{array}$ & $\begin{array}{c}\text { Ethyl- } \\
\text { benzene }\end{array}$ & $\begin{array}{c}\text { Hexachloro- } \\
\text { ethane }\end{array}$ & $\begin{array}{c}\text { Methylene } \\
\text { chloride }\end{array}$ \\
\hline 06/07/2002 & $06 / 28 / 2002$ & 109 & 95.7 & 96.9 & 95.1 & 111 & 87.9 & 91.5 \\
\hline $07 / 25 / 2002$ & 08/08/2002 & 72.4 & 65.7 & 103 & 91.2 & 106 & 85.8 & 92.3 \\
\hline $10 / 05 / 2002$ & $10 / 24 / 2002$ & 63.9 & 98.5 & 107 & 109 & 100 & 87.4 & 109 \\
\hline
\end{tabular}

\begin{tabular}{cccccccc}
\hline $\begin{array}{c}\text { Sampling } \\
\text { date }\end{array}$ & $\begin{array}{c}\text { Analyzed } \\
\text { date }\end{array}$ & $\begin{array}{c}\text { Tetra- } \\
\text { chloro- } \\
\text { ethene } \\
\text { (PCE) }\end{array}$ & $\begin{array}{c}\text { 1,1-Dichloro- } \\
\text { ethane }\end{array}$ & $\begin{array}{c}\text { 1,1-Dichlor- } \\
\text { oethene }\end{array}$ & $\begin{array}{c}\text { 1,1,1-Tri- } \\
\text { chloro- } \\
\text { ethane }\end{array}$ & $\begin{array}{c}\text { 1,1,2-Tri- } \\
\text { chloro- } \\
\text { ethane }\end{array}$ & $\begin{array}{c}\text { 1,1,2,2-Tetra- } \\
\text { chloro- } \\
\text { ethane }\end{array}$ \\
\hline $12 / 09 / 2001$ & $12 / 28 / 2001$ & 97.5 & 97.4 & 112 & 93.5 & 67.0 & 78.7 \\
$06 / 07 / 2002$ & $06 / 28 / 2002$ & 97.3 & 101 & 116 & 99.5 & 85.2 & 90.9 \\
$07 / 25 / 2002$ & $08 / 08 / 2002$ & 101 & 106 & 79.4 & 103 & 73.3 & 70.9 \\
$10 / 05 / 2002$ & $10 / 24 / 2002$ & 122 & 108 & 72.7 & 108 & 76.7 & 85.1 \\
$02 / 14 / 2003$ & $03 / 07 / 2003$ & 89.2 & 90.3 & 103 & 90.6 & 73.4 & 73.3 \\
benzene
\end{tabular}

\begin{tabular}{cccccccc}
\hline $\begin{array}{c}\text { Sampling } \\
\text { date }\end{array}$ & $\begin{array}{c}\text { Analyzed } \\
\text { date }\end{array}$ & $\begin{array}{c}\text { 1,2-Dichloro- } \\
\text { propane }\end{array}$ & $\begin{array}{c}\text { trans-1,2- } \\
\text { Dichloro- } \\
\text { ethene }\end{array}$ & $\begin{array}{c}\text { 1,2,4-Tri- } \\
\text { chloro- } \\
\text { benzene }\end{array}$ & $\begin{array}{c}\text { 1,3-Dichloro- } \\
\text { benzene }\end{array}$ & $\begin{array}{c}\text { 1,4-Dichloro- } \\
\text { benzene }\end{array}$ & $\begin{array}{c}\text { Naphthalene } \\
\text { Dichloro- } \\
\text { propene }\end{array}$ \\
\hline $12 / 09 / 2001$ & $12 / 28 / 2001$ & 75.5 & 72.3 & 89.2 & 90.5 & 91.2 & 94.8 \\
$06 / 07 / 2002$ & $06 / 28 / 2002$ & 105 & 123 & 91.7 & 90.4 & 95.7 & 69.7 \\
$07 / 25 / 2002$ & $08 / 08 / 2002$ & 103 & 85.2 & 99.0 & 104 & 107 & 104 \\
$10 / 05 / 2002$ & $10 / 24 / 2002$ & 118 & 87.4 & 107 & 107 & 110 & 121 \\
$02 / 14 / 2003$ & $03 / 07 / 2003$ & 84.2 & 80.8 & 87.2 & 89.2 & 88.5 & 95.7 \\
\end{tabular}

\begin{tabular}{|c|c|c|c|c|c|c|c|c|}
\hline $\begin{array}{l}\text { Sampling } \\
\text { date }\end{array}$ & $\begin{array}{l}\text { Analyzed } \\
\text { date }\end{array}$ & $\begin{array}{c}\text { cis-1,3- } \\
\text { Dichloro- } \\
\text { propene }\end{array}$ & $\begin{array}{l}\text { Trichlor- } \\
\text { oethene } \\
\text { (TCE) }\end{array}$ & $\begin{array}{c}\text { Methyl } \\
\text { methacrylate }\end{array}$ & $\begin{array}{c}\text { 1,2,3,4-Tetra- } \\
\text { methyl- } \\
\text { benzene }\end{array}$ & $\begin{array}{l}\text { Ethyl tert- } \\
\text { butyl ether } \\
\text { (ETBE) }\end{array}$ & $\begin{array}{l}\text { tert-Amyl } \\
\text { methyl ether } \\
\text { (TAME) }\end{array}$ & $\begin{array}{c}\text { trans-1,4- } \\
\text { Dichloro-2- } \\
\text { butene }\end{array}$ \\
\hline$\overline{12 / 09 / 2001}$ & $12 / 28 / 2001$ & 71.6 & 82.0 & 72.1 & 93.8 & 70.5 & 85.6 & 62.6 \\
\hline $07 / 25 / 2002$ & 08/08/2002 & 99.0 & 96.9 & 107 & 103 & 108 & 82.2 & 105 \\
\hline $10 / 05 / 2002$ & $10 / 24 / 2002$ & 116 & 103 & 109 & 110 & 113 & 92.9 & 112 \\
\hline $02 / 14 / 2003$ & 03/07/2003 & 72.9 & 91.0 & 91.2 & 94.9 & 95.2 & 75.9 & 94.7 \\
\hline
\end{tabular}


Table 22A. Quality-control results for volatile organic compounds with high breakthrough volumes in laboratory spikes for air samples collected at the Sweetwater Reservoir atmospheric site, San Diego County, California._Continued

[Values are given in percent recovery. Sample volume for quality assurance spike is 5 liters. Samples were processed at 20.0 degrees Celsius and 760 millimeters of mercury. Dates are in month/day/year format]

\begin{tabular}{|c|c|c|c|c|c|c|c|c|}
\hline $\begin{array}{l}\text { Sampling } \\
\text { date }\end{array}$ & $\begin{array}{l}\text { Analyzed } \\
\text { date }\end{array}$ & $\begin{array}{c}\text { Ethyl } \\
\text { meth- } \\
\text { acrylate }\end{array}$ & $\begin{array}{c}\text { Hexachloro- } \\
\text { butadiene }\end{array}$ & $\begin{array}{c}\text { Carbon } \\
\text { disulfide }\end{array}$ & $\begin{array}{c}\text { cis-1,2- } \\
\text { Dichloro- } \\
\text { ethene }\end{array}$ & $\begin{array}{c}\text { 2-Hexanone } \\
\text { (MBK) }\end{array}$ & $\begin{array}{l}\text { Ethenyl- } \\
\text { benzene } \\
\text { (Styrene) }\end{array}$ & o-Xylene \\
\hline 12/09/2001 & $12 / 28 / 2001$ & 94.8 & 76.9 & 78.5 & 97.4 & 73.0 & 106 & 99.8 \\
\hline $07 / 25 / 2002$ & $08 / 08 / 2002$ & 101 & 97.7 & 111 & 106 & 106 & 65.0 & 101 \\
\hline $10 / 05 / 2002$ & $10 / 24 / 2002$ & 113 & 106 & 110 & 105 & 107 & 75.6 & 108 \\
\hline $02 / 14 / 2003$ & 03/07/2003 & 96.6 & 92.8 & 91.7 & 89.5 & 89.0 & 82.0 & 97.7 \\
\hline
\end{tabular}

\begin{tabular}{|c|c|c|c|c|c|c|c|c|}
\hline $\begin{array}{l}\text { Sampling } \\
\text { date }\end{array}$ & $\begin{array}{l}\text { Analyzed } \\
\text { date }\end{array}$ & $\begin{array}{l}\text { 1,1-Dichloro- } \\
\text { propene }\end{array}$ & $\begin{array}{l}\text { 2,2-Dichloro- } \\
\text { propane }\end{array}$ & $\begin{array}{l}\text { 1,3-Dichloro- } \\
\text { propane }\end{array}$ & $\begin{array}{l}\text { 2-Ethyl- } \\
\text { toluene }\end{array}$ & $\begin{array}{c}\text { 1,2,3-Tri- } \\
\text { methyl- } \\
\text { benzene }\end{array}$ & $\begin{array}{l}\text { 1,2,4-Tri- } \\
\text { methyl- } \\
\text { benzene }\end{array}$ & $\begin{array}{l}\text { Isopropyl- } \\
\text { benzene } \\
\text { (Cumene) }\end{array}$ \\
\hline $12 / 09 / 2001$ & $12 / 28 / 2001$ & 71.7 & 75.8 & 93.9 & 95.2 & 96.7 & 91.2 & 90.1 \\
\hline $07 / 25 / 2002$ & 08/08/2002 & 114 & 101 & 111 & 111 & 109 & 92.5 & 91.9 \\
\hline $10 / 05 / 2002$ & $10 / 24 / 2002$ & 125 & 99.8 & 109 & 115 & 107 & 96.2 & 94.3 \\
\hline $02 / 14 / 2003$ & 03/07/2003 & 101 & 84.2 & 91.4 & 95.1 & 92.5 & 83.6 & 83.0 \\
\hline $\begin{array}{l}\text { Sampling } \\
\text { date }\end{array}$ & $\begin{array}{c}\text { Analyzed } \\
\text { date }\end{array}$ & $\begin{array}{l}n \text {-propyl- } \\
\text { benzene }\end{array}$ & $\begin{array}{l}\text { 1,3,5-Tri- } \\
\text { methyl- } \\
\text { benzene }\end{array}$ & $\begin{array}{l}\text { 1-Chloro- } \\
\text { 2-methyl- } \\
\text { benzene }\end{array}$ & $\begin{array}{l}\text { 1-Chloro- } \\
\text { 4-methyl- } \\
\text { benzene }\end{array}$ & $\begin{array}{l}\text { Bromo- } \\
\text { chloro- } \\
\text { methane }\end{array}$ & $\begin{array}{l}n \text {-Butyl- } \\
\text { benzene }\end{array}$ & $\begin{array}{c}\text { sec-Butyl- } \\
\text { benzene }\end{array}$ \\
\hline$\overline{12 / 09 / 2001}$ & $12 / 28 / 2001$ & 82.6 & 92.7 & 90.5 & 90.9 & 85.3 & 84.0 & 90.9 \\
\hline 06/07/2002 & $06 / 28 / 2002$ & 58.1 & 92.0 & 61.5 & 91.8 & 90.4 & 60.7 & 90.4 \\
\hline
\end{tabular}

\begin{tabular}{ccccccccc}
\hline $\begin{array}{c}\text { Sampling } \\
\text { date }\end{array}$ & $\begin{array}{c}\text { Analyzed } \\
\text { date }\end{array}$ & $\begin{array}{c}\text { tert-Butyl- } \\
\text { benzene }\end{array}$ & $\begin{array}{c}\text { 1-Isopropyl- } \\
\text { 4-methyl- } \\
\text { benzene }\end{array}$ & $\begin{array}{c}\text { 1,2,3-Tri- } \\
\text { chloro- } \\
\text { propane }\end{array}$ & $\begin{array}{c}\text { 1,1,1,2-Tetra- } \\
\text { chloro- } \\
\text { ethane }\end{array}$ & $\begin{array}{c}\text { 1,2,3-Tri- } \\
\text { chloro- } \\
\text { benzene }\end{array}$ & $\begin{array}{c}\text { 1,2-Dibromo- } \\
\text { ethane }\end{array}$ & $\begin{array}{c}\text { Methyl tert- } \\
\text { butyl ether } \\
\text { (MTBE) }\end{array}$ \\
\hline $12 / 09 / 2001$ & $12 / 28 / 2001$ & 91.5 & 91.2 & 90.7 & 91.8 & 93.5 & 93.4 \\
$06 / 07 / 2002$ & $06 / 28 / 2002$ & 89.9 & 92.2 & 90.5 & 92.4 & 95.6 & 94.1 \\
$07 / 25 / 2002$ & $08 / 08 / 2002$ & 93.0 & 95.8 & 94.2 & 96.1 & 97.8 & 95.4 \\
$10 / 05 / 2002$ & $10 / 24 / 2002$ & 98.1 & 98.8 & 97.1 & 97.2 & 100 & 101 \\
$02 / 14 / 2003$ & $03 / 07 / 2003$ & 85.1 & 87.7 & 84.3 & 85.6 & 86.1 & 86.1 \\
\end{tabular}

\begin{tabular}{cccccccc}
\hline $\begin{array}{c}\text { Sampling } \\
\text { date }\end{array}$ & $\begin{array}{c}\text { Analyzed } \\
\text { date }\end{array}$ & $\begin{array}{c}\text { 3-Chloro- } \\
\text { 1-propene }\end{array}$ & $\begin{array}{c}\text { 4-Methyl- } \\
\text { 2-pentanone } \\
\text { (MIBK) }\end{array}$ & Acetone & $\begin{array}{c}\text { Bromo- } \\
\text { benzene }\end{array}$ & Diethyl ether & $\begin{array}{c}\text { Diisopropyl } \\
\text { ether } \\
\text { (DIPE) }\end{array}$ \\
\hline $12 / 09 / 2001$ & $12 / 28 / 2001$ & 93.3 & 91.8 & 92.4 & 89.7 & 94.9 & 94.9 \\
acrylonitrile & Methyl \\
$06 / 07 / 2002$ & $06 / 28 / 2002$ & 97.5 & 94.2 & 94.2 & 92.2 & 95.6 & 92.7 \\
$07 / 25 / 2002$ & $08 / 08 / 2002$ & 100 & 98.2 & 81.6 & 79.5 & 85.0 & 82.0 \\
$10 / 05 / 2002$ & $10 / 24 / 2002$ & 99.2 & 99.5 & 95.2 & 96.4 & 99.9 & 98.9 \\
$02 / 14 / 2003$ & $03 / 07 / 2003$ & 82.6 & 87.5 & 82.8 & 83.7 & 83.7 & 84.1
\end{tabular}


Table 22A. Quality-control results for volatile organic compounds with high breakthrough volumes in laboratory spikes for air samples collected at the Sweetwater Reservoir atmospheric site, San Diego County, California.-Continued

[Values are given in percent recovery. Sample volume for quality assurance spike is 5 liters. Samples were processed at 20.0 degrees Celsius and 760 millimeters of mercury. Dates are in month/day/year format]

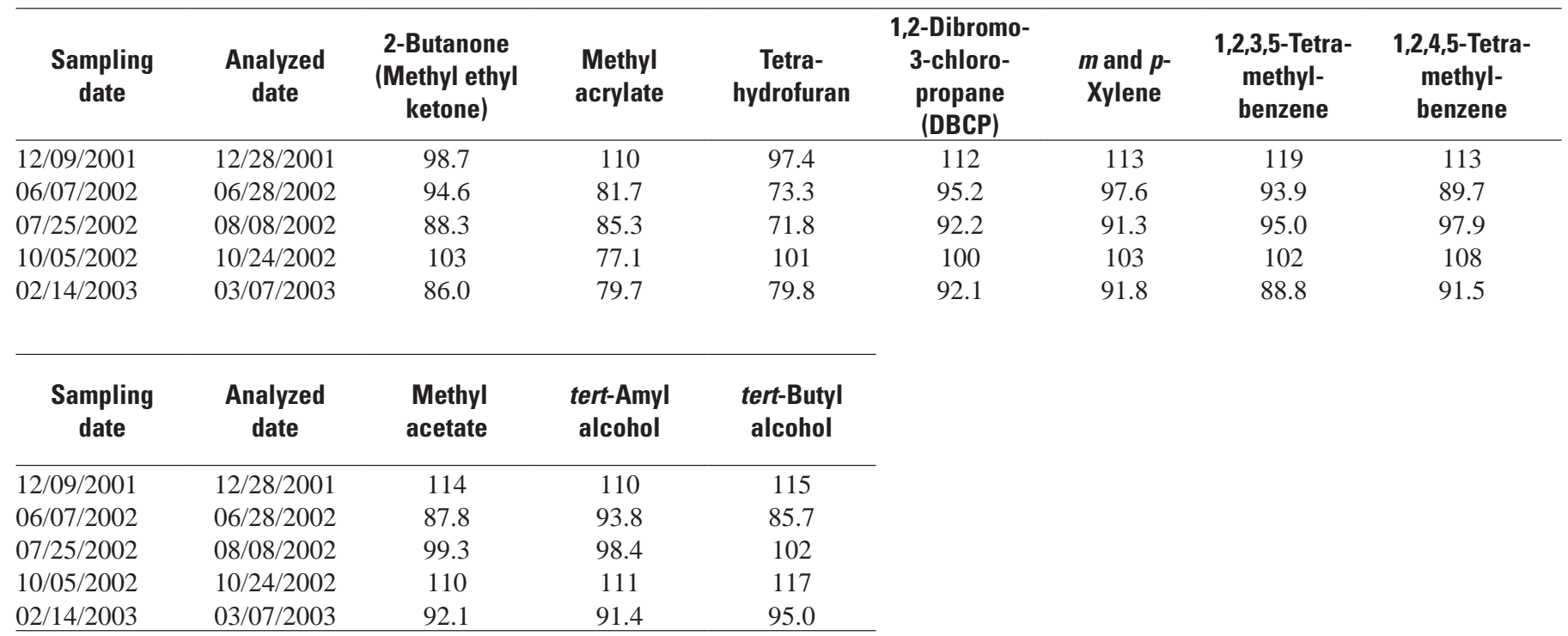


Table 22B. Quality-control results for volatile organic compounds with high breakthrough volumes in travel blanks for air samples collected at Sweetwater Reservoir atmospheric site at Sweetwater Dam, San Diego County, California.

[The site identification number is 324141117001601. Concentrations are given in parts per billion by volume (ppbv). Sample volume for quality assurance travel blank is 5.0 liters. Dates are in month/day/year format. Pairs of samples analyzed on the same date had been frozen and stored at the laboratory before analysis. ${ }^{\circ} \mathrm{C}$, degree Celsius; - , compound not detected at a concentration above laboratory reporting level. NA, not analyzed]

\begin{tabular}{|c|c|c|c|c|c|c|c|c|c|c|c|}
\hline $\begin{array}{l}\text { Sampling } \\
\text { date }\end{array}$ & $\begin{array}{c}\text { Analyzed } \\
\text { date }\end{array}$ & $\begin{array}{l}\text { Pressure } \\
\text { (mm of } \mathrm{Hg})\end{array}$ & $\begin{array}{c}\text { Temper- } \\
\text { ature } \\
\left({ }^{\circ} \mathrm{C}\right)\end{array}$ & $\begin{array}{l}\text { Bromo- } \\
\text { dichloro- } \\
\text { methane }\end{array}$ & Chloroform & Toluene & Benzene & $\begin{array}{c}\text { 2-Propene- } \\
\text { nitrile } \\
\text { (acrylo- } \\
\text { nitrile) }\end{array}$ & $\begin{array}{l}\text { Chloro- } \\
\text { benzene }\end{array}$ & $\begin{array}{c}\text { Ethyl- } \\
\text { benzene }\end{array}$ & $\begin{array}{c}\text { Methy- } \\
\text { lene } \\
\text { chloride }\end{array}$ \\
\hline $10 / 10 / 01$ & $10 / 26 / 01$ & 754 & 18.8 & NA & NA & NA & NA & NA & NA & NA & NA \\
\hline $10 / 22 / 01$ & $10 / 26 / 01$ & 753 & 17.8 & NA & NA & NA & NA & NA & NA & NA & $\mathrm{NA}$ \\
\hline 11/15/01 & $11 / 30 / 01$ & 753 & 15.7 & - & - & E0.01 & E0.10 & - & - & E0.01 & - \\
\hline $11 / 27 / 01$ & $11 / 30 / 01$ & 755 & 11.2 & - & E0.03 & E0.03 & E0.12 & - & - & E0.01 & E0.03 \\
\hline 12/09/01 & $12 / 28 / 01$ & 752 & 12.3 & NA & NA & NA & NA & NA & NA & NA & NA \\
\hline $12 / 21 / 01$ & $12 / 28 / 01$ & 758 & 11.4 & - & - & E0.01 & 0.21 & - & - & - & NA \\
\hline 01/03/02 & $01 / 22 / 02$ & 757 & 11.9 & - & - & E0.01 & 0.39 & E0.04 & - & - & E0.05 \\
\hline 01/14/02 & $01 / 22 / 02$ & 753 & 10.6 & - & - & E0.02 & 0.33 & E0.02 & - & - & E0.01 \\
\hline $01 / 28 / 02$ & $02 / 15 / 02$ & 754 & 10.6 & - & - & 0.15 & 0.33 & E0.01 & E0.01 & E0.03 & E0.04 \\
\hline 02/07/02 & $02 / 15 / 02$ & 761 & 10.2 & - & - & - & 0.44 & - & - & - & - \\
\hline 02/19/02 & 03/08/02 & 760 & 10.9 & - & - & E0.02 & 0.30 & E0.02 & - & E0.01 & - \\
\hline 03/03/02 & 03/08/02 & 761 & 13.9 & - & - & E0.02 & 0.31 & E0.03 & - & E0.01 & - \\
\hline 03/27/02 & $05 / 10 / 02$ & 755 & 12.5 & - & - & E0.01 & 0.19 & E0.02 & - & E0.01 & E0.01 \\
\hline $04 / 20 / 02$ & $05 / 10 / 02$ & 755 & 14.1 & - & - & E0.01 & E0.10 & - & - & E0.01 & - \\
\hline $05 / 14 / 02$ & $05 / 31 / 02$ & 751 & 17.0 & - & - & E0.01 & E0.05 & - & - & - & - \\
\hline $05 / 26 / 02$ & $05 / 31 / 02$ & 753 & 13.4 & - & - & E0.01 & 0.13 & - & - & - & - \\
\hline 06/07/02 & $06 / 28 / 02$ & 750 & 16.2 & - & - & E0.01 & E0.10 & - & - & - & - \\
\hline 06/19/02 & $06 / 28 / 02$ & 749 & 16.3 & - & - & E0.01 & E0.06 & - & - & - & - \\
\hline 07/01/02 & 07/19/02 & 753 & 16.9 & - & - & E0.02 & E0.07 & - & E0.01 & E0.01 & - \\
\hline 07/13/02 & $07 / 19 / 02$ & 751 & 18.1 & - & - & E0.01 & E0.12 & - & E0.01 & E0.01 & - \\
\hline $07 / 25 / 02$ & 08/08/02 & 754 & 18.7 & - & - & E0.01 & E0.10 & - & - & - & - \\
\hline 08/06/02 & 08/08/02 & 753 & 17.2 & - & - & E0.01 & E0.06 & - & - & - & - \\
\hline 08/18/02 & 09/06/02 & 752 & 18.5 & - & - & E0.01 & E0.06 & - & - & E0.01 & - \\
\hline 08/30/02 & 09/06/02 & 751 & 20.8 & - & - & E0.01 & E0.08 & - & - & E0.01 & - \\
\hline 09/11/02 & $09 / 27 / 02$ & 750 & 21.8 & - & - & E0.01 & 0.16 & E0.01 & - & E0.01 & - \\
\hline 09/23/02 & 09/27/02 & 749 & 22.7 & NA & NA & NA & $\mathrm{NA}$ & NA & NA & NA & NA \\
\hline $10 / 05 / 02$ & $10 / 24 / 02$ & 752 & 19.0 & - & - & E0.01 & E0.08 & - & - & E0.01 & - \\
\hline $10 / 17 / 02$ & $10 / 24 / 02$ & 752 & 16.2 & - & - & E0.02 & E0.09 & - & - & E0.01 & - \\
\hline $10 / 29 / 02$ & $11 / 13 / 02$ & 752 & 15.9 & - & - & E0.01 & E0.07 & - & - & E0.01 & - \\
\hline $11 / 10 / 02$ & $11 / 13 / 02$ & 756 & 16.1 & - & - & E0.01 & E0.06 & - & - & E0.01 & - \\
\hline $11 / 22 / 02$ & $12 / 13 / 02$ & 755 & 17.0 & - & - & E0.02 & E0.09 & - & - & E0.01 & - \\
\hline $12 / 04 / 02$ & $12 / 13 / 02$ & 758 & 13.6 & - & - & E0.02 & E0.06 & - & - & E0.01 & - \\
\hline $01 / 20 / 03$ & $02 / 07 / 03$ & 758 & 14.5 & - & - & E0.01 & E0.08 & - & - & E0.01 & - \\
\hline $02 / 04 / 03$ & 02/07/03 & 754 & 13.8 & - & - & E0.02 & E0.06 & - & - & E0.01 & - \\
\hline $02 / 14 / 03$ & 03/07/03 & 753 & 14.9 & - & - & E0.03 & 0.41 & E0.04 & - & E0.01 & - \\
\hline $02 / 14 / 03$ & 03/07/03 & NA & & E0.01 & - & - & - & - & - & - & - \\
\hline $02 / 26 / 03$ & 03/07/03 & 752 & 12.6 & - & - & E0.01 & E0.08 & E0.01 & - & E0.01 & - \\
\hline 03/10/03 & $03 / 28 / 03$ & 753 & 15.0 & - & - & E0.02 & E0.07 & - & - & - & - \\
\hline $03 / 22 / 03$ & 03/28/03 & 756 & 15.4 & - & - & E0.02 & 0.18 & - & - & - & - \\
\hline 04/03/03 & 04/18/03 & 757 & 12.4 & - & - & E0.02 & 0.15 & - & - & - & - \\
\hline $04 / 15 / 03$ & 04/18/03 & 757 & 12.6 & - & - & E0.01 & 0.21 & - & - & - & - \\
\hline 05/09/03 & $05 / 15 / 03$ & 762 & 13.8 & - & - & E0.01 & E0.08 & - & - & - & - \\
\hline $05 / 21 / 03$ & 06/06/03 & 758 & 20.2 & - & - & E0.01 & E0.10 & - & - & E0.01 & - \\
\hline 06/02/03 & 06/06/03 & 758 & 16.4 & - & - & E0.01 & E0.09 & - & - & E0.01 & - \\
\hline 06/14/03 & 06/30/03 & 759 & 19.1 & - & - & - & E0.05 & - & - & E0.01 & - \\
\hline $06 / 26 / 03$ & $06 / 30 / 03$ & 759 & 19.2 & - & - & E0.01 & E0.08 & - & - & - & - \\
\hline 07/09/03 & $07 / 25 / 03$ & 758 & 18.8 & - & - & E0.01 & E0.09 & - & - & - & - \\
\hline $07 / 20 / 03$ & $07 / 25 / 03$ & 761 & 22.9 & - & - & E0.01 & E0.12 & - & - & - & - \\
\hline 08/01/03 & $08 / 15 / 03$ & 762 & 23.0 & - & - & E0.01 & E0.07 & - & - & - & - \\
\hline 08/13/03 & $08 / 15 / 03$ & 758 & 23.6 & - & - & E0.01 & 0.17 & - & - & - & - \\
\hline 08/25/03 & $09 / 02 / 03$ & 759 & 23.0 & - & - & E0.03 & E0.09 & - & - & E0.01 & - \\
\hline 09/06/03 & $09 / 29 / 03$ & 759 & 24.4 & - & - & E0.01 & E0.11 & - & - & - & - \\
\hline 09/18/03 & $09 / 29 / 03$ & 757 & 19.5 & - & - & E0.01 & E0.08 & - & - & - & - \\
\hline 09/18/03 & $10 / 16 / 03$ & 760 & 19.6 & - & - & E0.02 & E0.12 & - & - & E0.01 & - \\
\hline
\end{tabular}


Table 22B. Quality-control results for volatile organic compounds with high breakthrough volumes in travel blanks for air samples collected at the Sweetwater Reservoir atmospheric site, San Diego County, California.-Continued

[The site identification number is 324141117001601 . Concentrations are given in parts per billion by volume (ppbv). Sample volume for quality assurance travel blank is 5.0 liters. Dates are in month/day/year format. Pairs of samples analyzed on the same date had been frozen and stored at the laboratory before analysis. ${ }^{\circ} \mathrm{C}$, degree Celsius; - , compound not detected at a concentration above laboratory reporting level; NA, not analyzed]

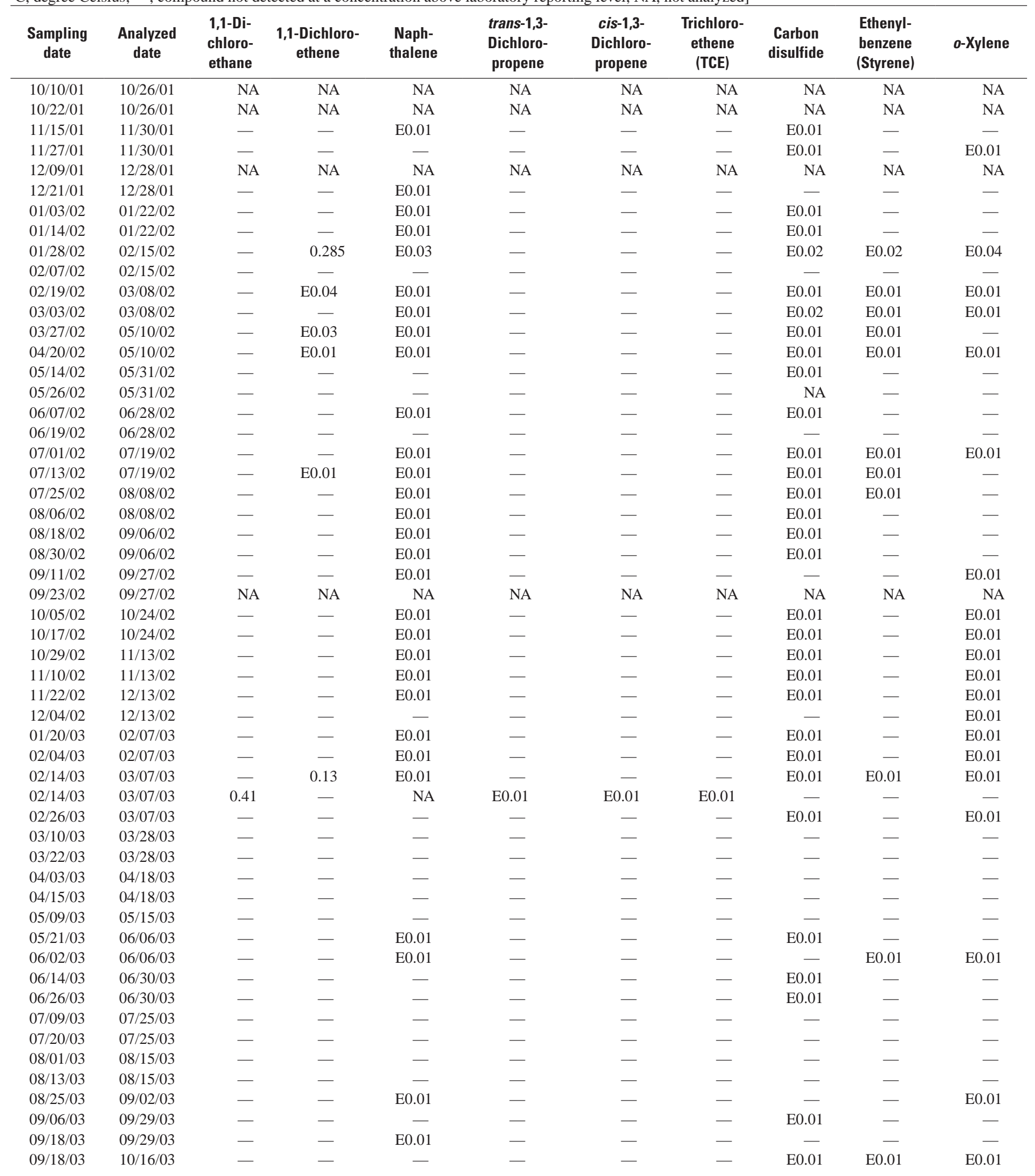


Table 22B. Quality-control results for volatile organic compounds with high breakthrough volumes in travel blanks for air samples collected at the Sweetwater Reservoir atmospheric site, San Diego County, California._Continued

[The site identification number is 324141117001601. Concentrations are given in parts per billion by volume (ppbv). Sample volume for quality assurance travel blank is 5.0 liters. Dates are in month/day/year format. Pairs of samples analyzed on the same date had been frozen and stored at the laboratory before analysis. ${ }^{\circ} \mathrm{C}$, degree Celsius; — , compound not detected at a concentration above laboratory reporting level; NA, not analyzed]

\begin{tabular}{|c|c|c|c|c|c|c|c|c|c|c|}
\hline $\begin{array}{l}\text { Sampling } \\
\text { date }\end{array}$ & $\begin{array}{l}\text { Analyzed } \\
\text { date }\end{array}$ & $\begin{array}{l}\text { 2-Ethyl- } \\
\text { toluene }\end{array}$ & $\begin{array}{c}\text { Methyl } \\
\text { tert- } \\
\text { butyl } \\
\text { ether } \\
\text { (MTBE) }\end{array}$ & $\begin{array}{l}\text { 3-Chloro- } \\
\text { 1-propene }\end{array}$ & Acetone & $\begin{array}{c}\text { 2-Butanone } \\
\text { (Methyl } \\
\text { ethyl } \\
\text { ketone) }\end{array}$ & $\begin{array}{c}m \text { - and } p \text { - } \\
\text { Xylene }\end{array}$ & $\begin{array}{l}\text { Methyl } \\
\text { acetate }\end{array}$ & $\begin{array}{c}\text { tert-Amyl } \\
\text { alcohol }\end{array}$ & $\begin{array}{l}\text { tert-Butyl } \\
\text { alcohol }\end{array}$ \\
\hline $10 / 10 / 01$ & $10 / 26 / 01$ & $\mathrm{NA}$ & NA & NA & NA & NA & NA & NA & $\mathrm{NA}$ & NA \\
\hline $10 / 22 / 01$ & $10 / 26 / 01$ & NA & NA & NA & NA & NA & NA & NA & NA & NA \\
\hline $11 / 15 / 01$ & $11 / 30 / 01$ & - & - & - & 1.02 & NA & E0.01 & - & - & - \\
\hline $11 / 27 / 01$ & $11 / 30 / 01$ & - & E0.01 & - & 0.61 & 0.167 & E0.01 & - & - & - \\
\hline $12 / 09 / 01$ & $12 / 28 / 01$ & NA & NA & NA & NA & NA & NA & NA & NA & NA \\
\hline $12 / 21 / 01$ & $12 / 28 / 01$ & - & - & - & 0.34 & - & - & - & - & - \\
\hline 01/03/02 & $01 / 22 / 02$ & - & - & - & 0.45 & - & - & 0.27 & - & - \\
\hline 01/14/02 & $01 / 22 / 02$ & - & - & - & 0.48 & - & E0.01 & 0.34 & - & - \\
\hline 01/28/02 & $02 / 15 / 02$ & - & E0.11 & - & 0.60 & - & 0.124 & 0.20 & - & - \\
\hline 02/07/02 & $02 / 15 / 02$ & - & - & - & 0.96 & - & - & - & - & - \\
\hline $02 / 19 / 02$ & 03/08/02 & - & - & - & 0.49 & - & E0.01 & 0.20 & - & - \\
\hline 03/03/02 & 03/08/02 & - & - & - & 1.49 & - & E0.01 & 0.36 & - & - \\
\hline $03 / 27 / 02$ & 05/10/02 & - & - & - & 0.79 & - & E0.01 & E0.15 & - & - \\
\hline $04 / 20 / 02$ & 05/10/02 & - & - & - & 0.65 & - & E0.01 & 0.30 & - & - \\
\hline $05 / 14 / 02$ & $05 / 31 / 02$ & - & - & - & E0.16 & - & - & E0.11 & - & E0.01 \\
\hline $05 / 26 / 02$ & $05 / 31 / 02$ & - & - & - & E0.15 & - & - & E0.11 & - & - \\
\hline 06/07/02 & 06/28/02 & - & - & - & 0.34 & - & - & - & - & - \\
\hline $06 / 19 / 02$ & $06 / 28 / 02$ & - & - & - & 0.29 & - & - & - & - & - \\
\hline 07/01/02 & 07/19/02 & - & - & - & 0.46 & E0.02 & E0.01 & - & - & - \\
\hline 07/13/02 & 07/19/02 & - & - & - & 0.52 & - & E0.01 & - & - & - \\
\hline $07 / 25 / 02$ & 08/08/02 & - & - & - & 0.36 & - & E0.01 & - & - & - \\
\hline 08/06/02 & 08/08/02 & - & - & - & 0.21 & - & E0.01 & - & - & - \\
\hline 08/18/02 & 09/06/02 & - & - & - & 0.23 & - & E0.01 & - & - & - \\
\hline 08/30/02 & 09/06/02 & - & - & - & 0.20 & - & E0.01 & - & - & - \\
\hline $09 / 11 / 02$ & $09 / 27 / 02$ & - & - & E0.01 & E0.16 & - & E0.01 & - & - & - \\
\hline 09/23/02 & $09 / 27 / 02$ & NA & NA & NA & NA & NA & NA & NA & NA & NA \\
\hline $10 / 05 / 02$ & $10 / 24 / 02$ & - & - & - & E0.09 & - & E0.01 & - & - & - \\
\hline $10 / 17 / 02$ & $10 / 24 / 02$ & - & - & - & 0.46 & - & E0.01 & - & - & - \\
\hline $10 / 29 / 02$ & $11 / 13 / 02$ & - & - & - & 0.36 & - & E0.01 & - & - & - \\
\hline $11 / 10 / 02$ & $11 / 13 / 02$ & - & - & - & E0.10 & - & E0.01 & - & - & - \\
\hline $11 / 22 / 02$ & $12 / 13 / 02$ & - & - & - & 0.45 & - & E0.01 & - & - & - \\
\hline $12 / 04 / 02$ & $12 / 13 / 02$ & - & - & - & 0.27 & - & E0.01 & - & - & - \\
\hline 01/20/03 & 02/07/03 & - & - & - & 0.19 & - & E0.01 & - & - & - \\
\hline $02 / 04 / 03$ & 02/07/03 & - & - & - & 0.30 & - & E0.01 & - & - & - \\
\hline $02 / 14 / 03$ & 03/07/03 & - & - & - & 0.26 & - & E0.01 & - & - & - \\
\hline $02 / 14 / 03$ & 03/07/03 & E0.01 & - & - & - & - & - & - & 0.03 & - \\
\hline $02 / 26 / 03$ & 03/07/03 & - & - & - & E0.15 & - & E0.01 & - & - & - \\
\hline $03 / 10 / 03$ & $03 / 28 / 03$ & - & - & - & 0.35 & - & - & - & - & - \\
\hline $03 / 22 / 03$ & $03 / 28 / 03$ & - & - & - & 0.46 & - & - & - & - & - \\
\hline $04 / 03 / 03$ & $04 / 18 / 03$ & - & - & - & 0.26 & - & - & - & - & - \\
\hline $04 / 15 / 03$ & $04 / 18 / 03$ & - & - & - & E0.12 & - & - & - & - & - \\
\hline $05 / 09 / 03$ & $05 / 15 / 03$ & - & - & - & E0.11 & - & - & - & - & - \\
\hline $05 / 21 / 03$ & 06/06/03 & - & - & - & 0.29 & - & E0.01 & - & - & - \\
\hline $06 / 02 / 03$ & 06/06/03 & - & - & - & 0.28 & - & E0.01 & - & - & - \\
\hline $06 / 14 / 03$ & $06 / 30 / 03$ & - & - & - & 0.59 & - & E0.01 & - & - & - \\
\hline $06 / 26 / 03$ & $06 / 30 / 03$ & - & - & - & 0.27 & - & - & - & - & - \\
\hline 07/09/03 & $07 / 25 / 03$ & - & - & - & 0.35 & - & - & - & - & - \\
\hline $07 / 20 / 03$ & $07 / 25 / 03$ & - & - & - & 0.23 & - & - & - & - & - \\
\hline 08/01/03 & 08/15/03 & - & - & - & 0.25 & - & - & - & - & - \\
\hline 08/13/03 & 08/15/03 & - & - & - & 0.68 & - & - & - & - & - \\
\hline 08/25/03 & 09/02/03 & - & - & - & 0.61 & - & E0.01 & - & - & - \\
\hline 09/06/03 & $09 / 29 / 03$ & - & - & - & E0.17 & - & - & - & - & - \\
\hline 09/18/03 & $09 / 29 / 03$ & - & - & - & E0.16 & - & E0.01 & - & - & - \\
\hline $09 / 18 / 03$ & $10 / 16 / 03$ & - & - & - & 0.61 & - & E0.01 & - & - & - \\
\hline
\end{tabular}




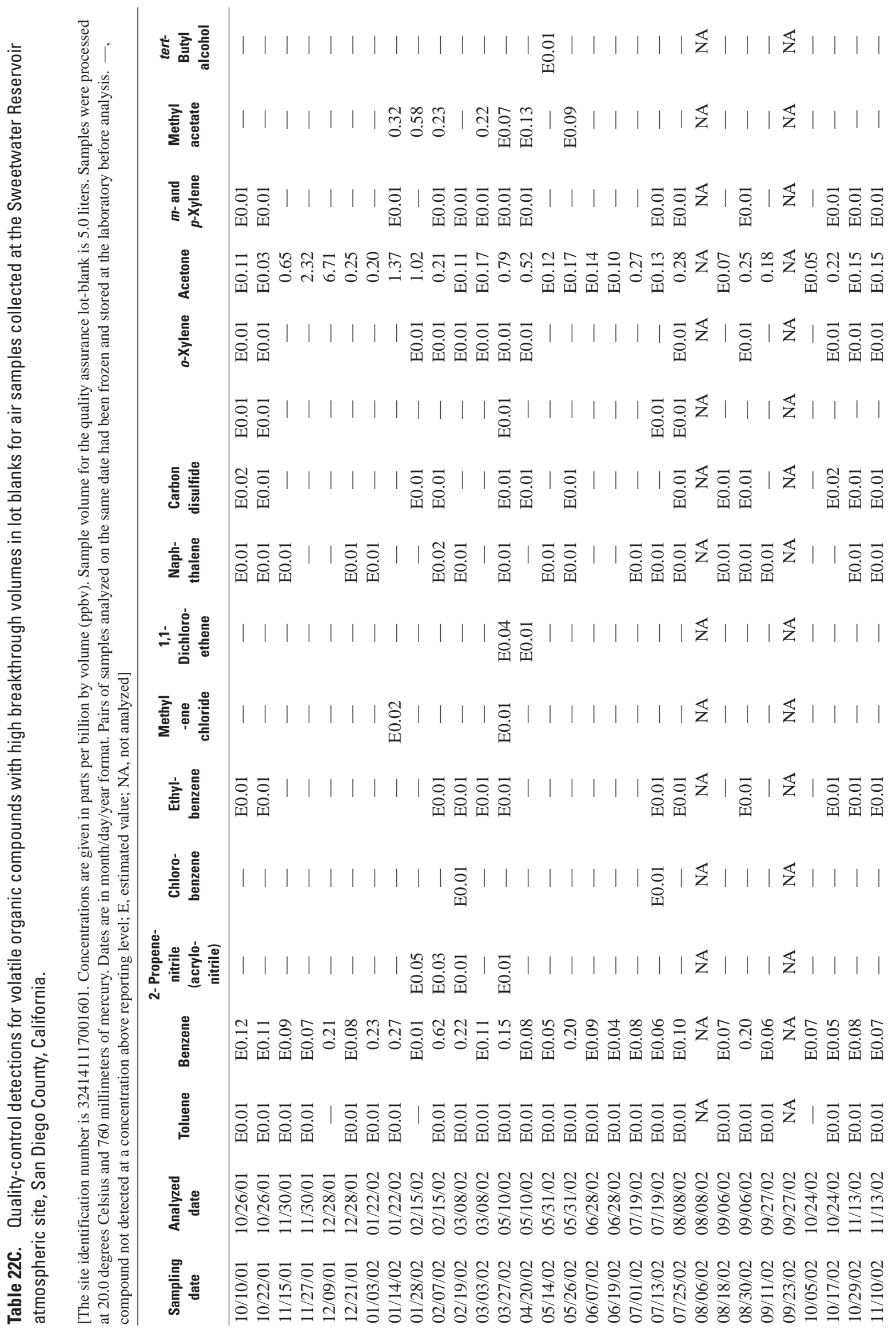




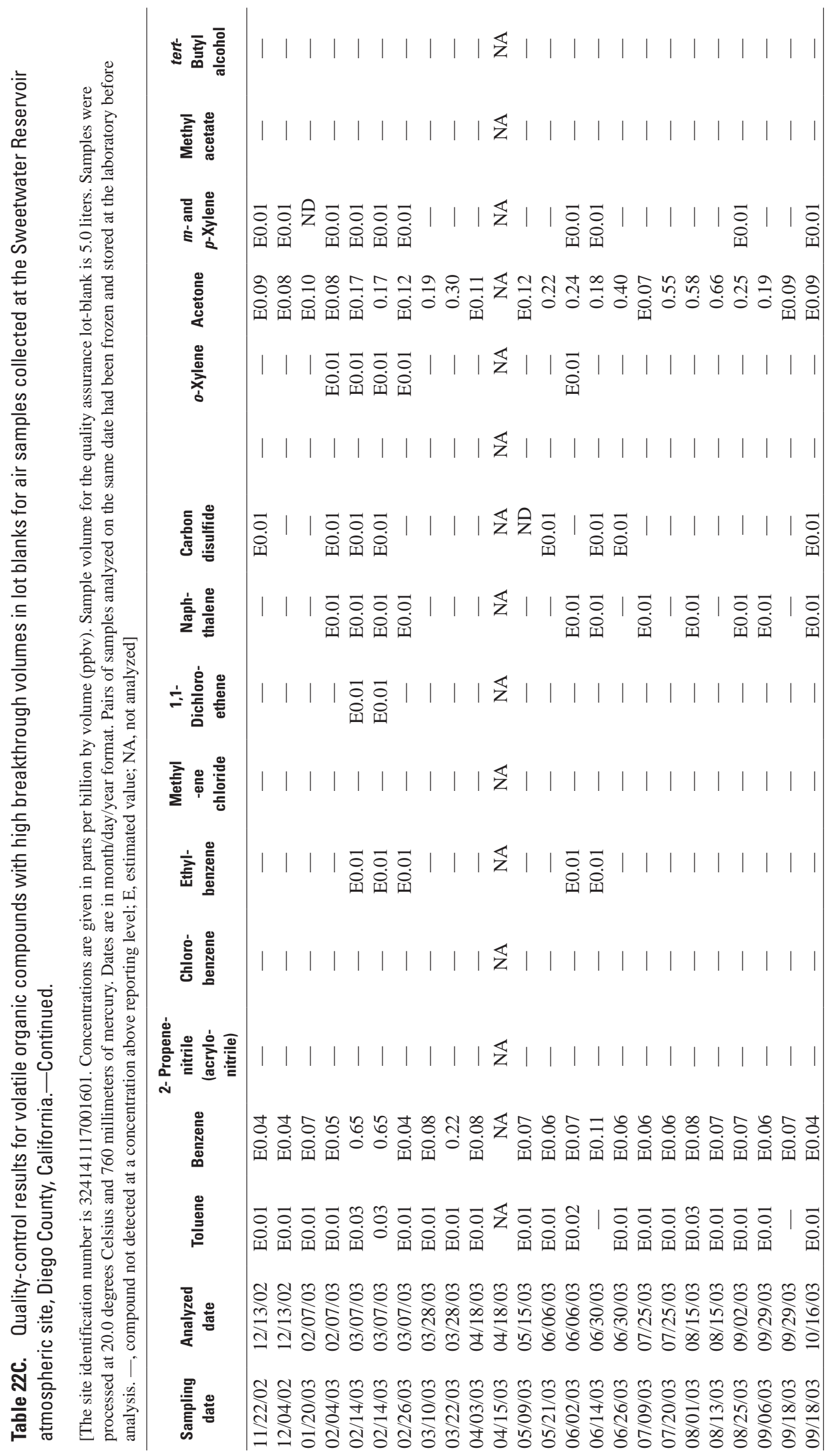


Table 22D. Quality-control detections for volatile organic compounds with high breakthrough volumes in laboratory blanks for air samples collected at the Sweetwater Reservoir atmospheric site, San Diego County, California.

[The site identification number is 324141117001601 . Concentrations are given in parts per billion by volume (ppbv). Sample volume for the quality assurance lot-blank is 5.0 liters. Samples were processed at 20.0 degrees Celsius and 760 millimeters of mercury. Dates are in month/day/year format. Pairs of samples analyzed on the same date had been frozen and stored at the laboratory before analysis. - , compound not detected at a concentration above reporting level; E, estimated value; NA, not analyzed]

\begin{tabular}{|c|c|c|c|c|c|c|c|c|c|}
\hline $\begin{array}{l}\text { Sampling } \\
\text { date }\end{array}$ & $\begin{array}{c}\text { Analyzed } \\
\text { date }\end{array}$ & Toluene & Benzene & $\begin{array}{c}\text { 2-Propene-nitrile } \\
\text { (Acrylo- } \\
\text { nitrile) }\end{array}$ & $\begin{array}{c}\text { Chloro- } \\
\text { benzene }\end{array}$ & $\begin{array}{c}\text { Ethyl- } \\
\text { benzene }\end{array}$ & $\begin{array}{c}\text { Methylene } \\
\text { chloride }\end{array}$ & $\begin{array}{l}\text { 1,1-Di- } \\
\text { chloro- } \\
\text { ethene }\end{array}$ & $\begin{array}{c}\text { 1,1,2,2- } \\
\text { Tetra- } \\
\text { chloro- } \\
\text { ethane }\end{array}$ \\
\hline $10 / 10 / 01$ & $10 / 26 / 01$ & NA & NA & NA & NA & NA & NA & NA & NA \\
\hline $10 / 22 / 01$ & $10 / 26 / 01$ & NA & NA & NA & NA & NA & NA & NA & NA \\
\hline $11 / 27 / 01$ & $11 / 30 / 01$ & E0.01 & E0.10 & - & - & - & - & - & - \\
\hline $11 / 15 / 01$ & $11 / 30 / 01$ & E0.01 & E0.09 & - & - & - & - & - & - \\
\hline $12 / 09 / 01$ & $12 / 28 / 01$ & E0.02 & 0.47 & - & - & E0.01 & - & - & - \\
\hline $12 / 21 / 01$ & $12 / 28 / 01$ & E0.01 & 0.14 & - & - & - & - & - & - \\
\hline 01/03/02 & $01 / 22 / 02$ & E0.01 & 0.28 & E0.04 & - & - & 0.12 & - & - \\
\hline $01 / 14 / 02$ & $01 / 22 / 02$ & E0.02 & 0.61 & E0.05 & - & - & 0.10 & - & - \\
\hline $01 / 28 / 02$ & $02 / 15 / 02$ & E0.01 & 0.15 & E0.01 & - & - & - & - & - \\
\hline 02/07/02 & $02 / 15 / 02$ & E0.01 & 1.14 & ND & - & - & - & - & - \\
\hline 02/19/02 & 03/08/02 & E0.01 & 0.28 & E0.01 & - & E0.01 & - & - & - \\
\hline 03/03/02 & 03/08/02 & E0.01 & 0.17 & E0.01 & - & E0.01 & - & - & - \\
\hline 03/27/02 & 05/10/02 & E0.01 & 0.20 & E0.01 & E0.01 & - & - & E0.01 & - \\
\hline $04 / 20 / 02$ & 05/10/02 & E0.01 & E0.05 & - & - & - & - & E0.01 & - \\
\hline $05 / 14 / 02$ & $05 / 31 / 02$ & E0.01 & E0.04 & - & - & - & - & E0.01 & - \\
\hline $05 / 26 / 02$ & $05 / 31 / 02$ & E0.01 & 0.26 & - & - & - & - & - & - \\
\hline 06/07/02 & $06 / 28 / 02$ & E0.01 & E0.07 & - & - & - & - & - & - \\
\hline $06 / 19 / 02$ & $06 / 28 / 02$ & E0.01 & E0.07 & - & - & - & - & - & - \\
\hline 07/01/02 & 07/19/02 & E0.01 & E0.11 & - & - & E0.01 & - & - & - \\
\hline $07 / 13 / 02$ & 07/19/02 & E0.01 & 0.14 & - & E0.01 & - & - & - & - \\
\hline $07 / 25 / 02$ & 08/08/02 & E0.01 & 0.17 & - & - & E0.01 & - & - & - \\
\hline 08/06/02 & 08/08/02 & E0.01 & 0.18 & - & - & E0.01 & - & - & - \\
\hline 08/18/02 & $09 / 06 / 02$ & E0.01 & E0.07 & - & - & E0.01 & - & - & E0.01 \\
\hline 08/30/02 & $09 / 06 / 02$ & E0.01 & 0.19 & - & - & E0.01 & - & - & - \\
\hline $09 / 11 / 02$ & $09 / 27 / 02$ & E0.01 & 0.13 & E0.01 & - & E0.01 & - & - & - \\
\hline 09/23/02 & $09 / 27 / 02$ & NA & NA & NA & NA & NA & NA & NA & NA \\
\hline $10 / 05 / 02$ & $10 / 24 / 02$ & E0.01 & E0.06 & - & - & E0.01 & - & - & - \\
\hline $10 / 17 / 02$ & $10 / 24 / 02$ & E0.01 & E0.08 & - & - & E0.01 & - & - & - \\
\hline $10 / 29 / 02$ & $11 / 13 / 02$ & E0.01 & E0.09 & - & - & E0.01 & - & - & - \\
\hline $11 / 10 / 02$ & $11 / 13 / 02$ & E0.02 & E0.09 & - & - & E0.01 & - & - & - \\
\hline $11 / 22 / 02$ & $12 / 13 / 02$ & E0.01 & E0.12 & - & - & E0.01 & - & - & - \\
\hline $12 / 04 / 02$ & $12 / 13 / 02$ & E0.01 & E0.09 & - & - & E0.01 & - & - & - \\
\hline $01 / 20 / 03$ & 02/07/03 & E0.02 & E0.09 & - & - & E0.01 & - & - & - \\
\hline $02 / 04 / 03$ & 02/07/03 & E0.01 & E0.09 & - & - & E0.01 & - & - & - \\
\hline $02 / 14 / 03$ & 03/07/03 & E0.01 & 0.81 & E0.02 & - & E0.01 & - & - & - \\
\hline 02/14/03 & 03/07/03 & E0.01 & 0.81 & E0.02 & - & E0.01 & - & - & - \\
\hline $02 / 26 / 03$ & 03/07/03 & E0.01 & 0.30 & E0.01 & - & E0.01 & - & - & - \\
\hline 03/10/03 & 03/28/03 & E0.01 & E0.12 & - & - & - & - & - & - \\
\hline $03 / 22 / 03$ & $03 / 28 / 03$ & E0.01 & E0.10 & - & - & - & - & - & - \\
\hline 04/03/03 & 04/18/03 & E0.01 & 0.13 & - & - & - & - & - & - \\
\hline $04 / 15 / 03$ & 04/18/03 & NA & NA & NA & NA & NA & NA & NA & NA \\
\hline 05/09/03 & $05 / 15 / 03$ & E0.01 & E0.06 & - & - & - & - & - & - \\
\hline $05 / 21 / 03$ & 06/06/03 & E0.01 & E0.08 & - & - & E0.01 & - & - & - \\
\hline $06 / 02 / 03$ & 06/06/03 & E0.01 & E0.08 & - & - & E0.01 & - & - & - \\
\hline $06 / 14 / 03$ & $06 / 30 / 03$ & - & 0.18 & - & - & - & - & - & - \\
\hline $06 / 26 / 03$ & $06 / 30 / 03$ & E0.01 & E0.09 & - & - & - & - & - & - \\
\hline 07/09/03 & $07 / 25 / 03$ & E0.01 & 0.15 & - & - & - & - & - & - \\
\hline $07 / 20 / 03$ & $07 / 25 / 03$ & E0.01 & E0.08 & - & - & - & - & - & - \\
\hline 08/01/03 & 08/15/03 & E0.01 & E0.06 & - & - & - & - & - & - \\
\hline 08/13/03 & 08/15/03 & E0.01 & E0.08 & - & - & - & - & - & - \\
\hline $08 / 25 / 03$ & $09 / 02 / 03$ & E0.01 & E0.08 & - & - & - & - & - & - \\
\hline 09/06/03 & 09/29/03 & E0.01 & 0.13 & - & - & - & - & - & - \\
\hline 09/18/03 & $09 / 29 / 03$ & E0.01 & E0.06 & - & - & - & - & - & - \\
\hline 09/18/03 & $10 / 16 / 03$ & E0.01 & 0.18 & - & - & E0.01 & - & - & - \\
\hline
\end{tabular}


Table 22D. Quality-control results for volatile organic compounds with high breakthrough volumes in laboratory blanks for air samples collected at the Sweetwater Reservoir atmospheric site, San Diego County, California.-Continued

[The site identification number is 324141117001601. Concentrations are given in parts per billion by volume (ppbv). Sample volume for the quality assurance laboratory-blank is 5.0 liters. Samples were processed at 20.0 degrees Celsius and 760 millimeters of mercury. Dates are in month/day/year format. Pairs of samples analyzed on the same date had been frozen and stored at the laboratory before analysis. —, compound not detected at a concentration above reporting level; E, estimated value; NA, not analyzed]

\begin{tabular}{|c|c|c|c|c|c|c|c|c|c|c|}
\hline $\begin{array}{c}\text { Sampling } \\
\text { date }\end{array}$ & $\begin{array}{l}\text { Analyzed } \\
\text { date }\end{array}$ & $\begin{array}{l}\text { Naph- } \\
\text { thalene }\end{array}$ & $\begin{array}{c}\text { Carbon } \\
\text { disulfide }\end{array}$ & $\begin{array}{l}\text { Ethenyl- } \\
\text { benzene } \\
\text { (Styrene) }\end{array}$ & o-Xylene & $\begin{array}{l}\text { 3-Chloro- } \\
\text { 1-propene }\end{array}$ & Acetone & $\begin{array}{c}\text { 2-Butanone } \\
\text { (Methyl } \\
\text { ethyl } \\
\text { ketone) }\end{array}$ & $\begin{array}{c}m \text { - and } \\
p \text {-Xylene }\end{array}$ & $\begin{array}{l}\text { Methyl } \\
\text { acetate }\end{array}$ \\
\hline $10 / 10 / 01$ & $10 / 26 / 01$ & NA & NA & NA & NA & NA & NA & NA & NA & NA \\
\hline $10 / 22 / 01$ & $10 / 26 / 01$ & NA & NA & NA & NA & NA & NA & NA & NA & NA \\
\hline $11 / 27 / 01$ & $11 / 30 / 01$ & - & E0.01 & - & - & - & 0.69 & - & - & - \\
\hline $11 / 15 / 01$ & $11 / 30 / 01$ & - & E0.01 & - & - & - & 1.66 & - & - & - \\
\hline $12 / 09 / 01$ & $12 / 28 / 01$ & E0.01 & E0.02 & E0.01 & E0.01 & - & 0.37 & - & E0.01 & - \\
\hline $12 / 21 / 01$ & $12 / 28 / 01$ & E0.01 & - & - & - & - & 0.28 & - & - & - \\
\hline 01/03/02 & $01 / 22 / 02$ & E0.01 & - & - & - & - & 0.69 & - & - & 0.41 \\
\hline 01/14/02 & $01 / 22 / 02$ & E0.01 & E0.02 & E0.01 & - & - & 0.47 & - & E0.01 & 0.31 \\
\hline $01 / 28 / 02$ & $02 / 15 / 02$ & - & - & - & E0.01 & - & 1.00 & - & - & 0.23 \\
\hline 02/07/02 & $02 / 15 / 02$ & E0.01 & - & - & - & - & 1.11 & - & - & E0.10 \\
\hline $02 / 19 / 02$ & 03/08/02 & E0.01 & - & - & E0.01 & - & E0.12 & - & E0.01 & E0.10 \\
\hline 03/03/02 & 03/08/02 & - & - & - & E0.01 & - & 0.79 & - & E0.01 & 0.19 \\
\hline $03 / 27 / 02$ & 05/10/02 & E0.01 & - & - & E0.01 & - & E0.17 & - & E0.01 & 0.17 \\
\hline $04 / 20 / 02$ & 05/10/02 & E0.01 & E0.01 & - & - & - & E0.11 & - & E0.01 & E0.14 \\
\hline 05/14/02 & $05 / 31 / 02$ & - & E0.01 & - & - & - & 0.20 & - & - & E0.09 \\
\hline $05 / 26 / 02$ & $05 / 31 / 02$ & E0.01 & E0.01 & - & - & - & 0.24 & - & - & 0.19 \\
\hline 06/07/02 & $06 / 28 / 02$ & E0.01 & E0.02 & - & - & - & E0.15 & - & - & - \\
\hline 06/19/02 & $06 / 28 / 02$ & - & - & - & - & - & E0.15 & - & - & - \\
\hline 07/01/02 & 07/19/02 & E0.01 & E0.01 & E0.01 & E0.01 & - & 0.46 & E0.02 & E0.01 & - \\
\hline 07/13/02 & 07/19/02 & E0.01 & E0.01 & E0.01 & - & - & 0.40 & - & E0.01 & - \\
\hline $07 / 25 / 02$ & 08/08/02 & E0.01 & E0.01 & E0.01 & E0.01 & - & 0.37 & - & E0.01 & - \\
\hline 08/06/02 & 08/08/02 & E0.01 & - & - & E0.01 & - & 0.25 & - & E0.01 & - \\
\hline $08 / 18 / 02$ & $09 / 06 / 02$ & E0.01 & - & - & - & - & 0.20 & - & E0.01 & - \\
\hline 08/30/02 & $09 / 06 / 02$ & E0.01 & E0.01 & - & E0.01 & - & E 0.17 & - & E0.01 & - \\
\hline 09/11/02 & $09 / 27 / 02$ & E0.01 & - & - & E0.01 & E0.01 & E0.10 & - & E0.01 & - \\
\hline 09/23/02 & $09 / 27 / 02$ & NA & NA & NA & NA & NA & NA & NA & NA & NA \\
\hline $10 / 05 / 02$ & $10 / 24 / 02$ & E0.01 & E0.01 & - & E0.01 & - & E0.16 & - & E0.01 & - \\
\hline $10 / 17 / 02$ & $10 / 24 / 02$ & E0.01 & - & - & E0.01 & - & E0.09 & - & E0.01 & - \\
\hline $10 / 29 / 02$ & $11 / 13 / 02$ & E0.01 & E0.01 & - & E0.01 & - & 0.70 & - & E0.01 & - \\
\hline $11 / 10 / 02$ & $11 / 13 / 02$ & E0.02 & E0.02 & E0.01 & E0.01 & - & E0.08 & - & E0.02 & - \\
\hline $11 / 22 / 02$ & $12 / 13 / 02$ & E0.01 & E0.01 & - & E0.01 & - & 0.52 & - & E0.01 & - \\
\hline $12 / 04 / 02$ & $12 / 13 / 02$ & - & - & - & - & - & E0.11 & - & E0.01 & - \\
\hline $01 / 20 / 03$ & 02/07/03 & E0.01 & E0.01 & E0.01 & E0.01 & - & 0.22 & - & E0.01 & - \\
\hline 02/04/03 & $02 / 07 / 03$ & E0.01 & - & - & E0.01 & - & E0.08 & - & E0.01 & - \\
\hline $02 / 14 / 03$ & 03/07/03 & E0.01 & E0.01 & - & E0.01 & - & E0.11 & - & E0.01 & - \\
\hline $02 / 14 / 03$ & 03/07/03 & E0.01 & E0.01 & - & E0.01 & - & E0.11 & - & E0.01 & - \\
\hline $02 / 26 / 03$ & 03/07/03 & E0.01 & E0.01 & - & E0.01 & - & 0.55 & - & E0.01 & - \\
\hline 03/10/03 & 03/28/03 & - & - & - & - & - & 0.27 & - & - & - \\
\hline $03 / 22 / 03$ & $03 / 28 / 03$ & - & - & - & - & - & 0.25 & - & - & - \\
\hline $04 / 03 / 03$ & $04 / 18 / 03$ & - & - & - & - & - & E0.10 & - & - & - \\
\hline $04 / 15 / 03$ & 04/18/03 & NA & NA & NA & NA & NA & NA & NA & NA & NA \\
\hline 05/09/03 & $05 / 15 / 03$ & E0.01 & - & - & - & - & E0.10 & - & - & - \\
\hline $05 / 21 / 03$ & $06 / 06 / 03$ & E0.01 & E0.01 & E0.01 & - & - & 0.26 & - & E0.01 & - \\
\hline $06 / 02 / 03$ & $06 / 06 / 03$ & E0.01 & E0.01 & - & E0.01 & - & 0.26 & - & E0.01 & - \\
\hline $06 / 14 / 03$ & 06/30/03 & E0.01 & E0.01 & - & - & - & 0.19 & - & E0.01 & - \\
\hline 06/26/03 & $06 / 30 / 03$ & E0.01 & E0.01 & - & - & - & 0.31 & - & - & - \\
\hline 07/09/03 & $07 / 25 / 03$ & - & - & - & - & - & E0.18 & - & - & - \\
\hline $07 / 20 / 03$ & $07 / 25 / 03$ & - & - & - & - & - & 0.19 & - & - & - \\
\hline 08/01/03 & $08 / 15 / 03$ & - & - & - & - & - & E0.07 & - & - & - \\
\hline 08/13/03 & 08/15/03 & - & - & - & - & - & 0.45 & - & - & - \\
\hline $08 / 25 / 03$ & $09 / 02 / 03$ & E0.01 & - & - & - & - & E0.16 & - & E0.01 & - \\
\hline 09/06/03 & $09 / 29 / 03$ & E0.01 & - & - & - & - & E0.13 & - & - & - \\
\hline 09/18/03 & $09 / 29 / 03$ & E0.01 & E0.01 & - & - & - & 0.30 & - & E0.01 & - \\
\hline 09/18/03 & $10 / 16 / 03$ & E0.01 & - & - & E0.01 & - & E0.14 & - & E0.01 & - \\
\hline
\end{tabular}


Table 23A. Quality-control results for laboratory and field reagent blanks and laboratory solvent blanks for polycyclic aromatic hydrocarbon (PAH) compound concentrations from the Sweetwater Reservoir atmospheric site, San Diego County, California.

[All blank analyses by gas chromatography/mass spectrometry with selected ion monitoring (SIM). Field blank identification number is 324141117001601. Concentrations are given in nanograms per cubic meter $\left(\mathrm{ng} / \mathrm{m}^{3}\right)$ assuming a $315-\mathrm{m}^{3}$ sample volume unless noted. GFF, glass fiber filter; PSE, pressurized solvent extraction; PUF, polyurethane foam plug; _, compound not detected at a concentration above laboratory reporting level]

\begin{tabular}{|c|c|c|c|c|c|c|c|c|}
\hline $\begin{array}{l}\text { Begin date } \\
\text { (mm/dd/yyyy) }\end{array}$ & $\begin{array}{c}\text { Sample } \\
\text { type }\end{array}$ & $\begin{array}{c}\text { Sample } \\
\text { component } \\
\text { used }\end{array}$ & $\begin{array}{c}\text { Sample } \\
\text { preparation set } \\
\text { number }\end{array}$ & $\begin{array}{c}\text { Extraction } \\
\text { by }\end{array}$ & $\begin{array}{c}\text { Extract } \\
\text { split }\end{array}$ & Anthracene & $\begin{array}{c}\text { Benz[a] } \\
\text { anthracene }\end{array}$ & $\begin{array}{c}\text { Benzo[ghi] } \\
\text { perylene }\end{array}$ \\
\hline $08 / 25 / 2002$ & Lab blank & $\begin{array}{l}\text { Clean, used field } \\
\text { PUF }\end{array}$ & 02.037 & Soxhlet & No & 0.026 & - & - \\
\hline 08/25/2002 & $\begin{array}{l}\text { Lab solvent } \\
\text { blank }\end{array}$ & Solvent only & 02.037 & Soxhlet & No & - & - & - \\
\hline $12 / 16 / 2002$ & Lab blank & GFF & 02.35009 & PSE & No & - & - & - \\
\hline $12 / 16 / 2002$ & Lab blank & New PUF & 02.35009 & PSE & No & - & - & - \\
\hline $11 / 19 / 2003$ & Lab blank & GFF & 02.35008 & PSE & Yes & - & - & - \\
\hline $11 / 19 / 2003$ & Lab blank & New PUF & 02.35008 & PSE & Yes & - & - & - \\
\hline 06/03/2003 & Field blank & GFF & 02.35008 & PSE & Yes & - & - & - \\
\hline 06/03/2003 & Field blank & Top PUF & 02.35008 & PSE & Yes & - & - & - \\
\hline 06/03/2003 & Field blank & Bottom PUF & 02.35008 & PSE & Yes & - & - & - \\
\hline
\end{tabular}

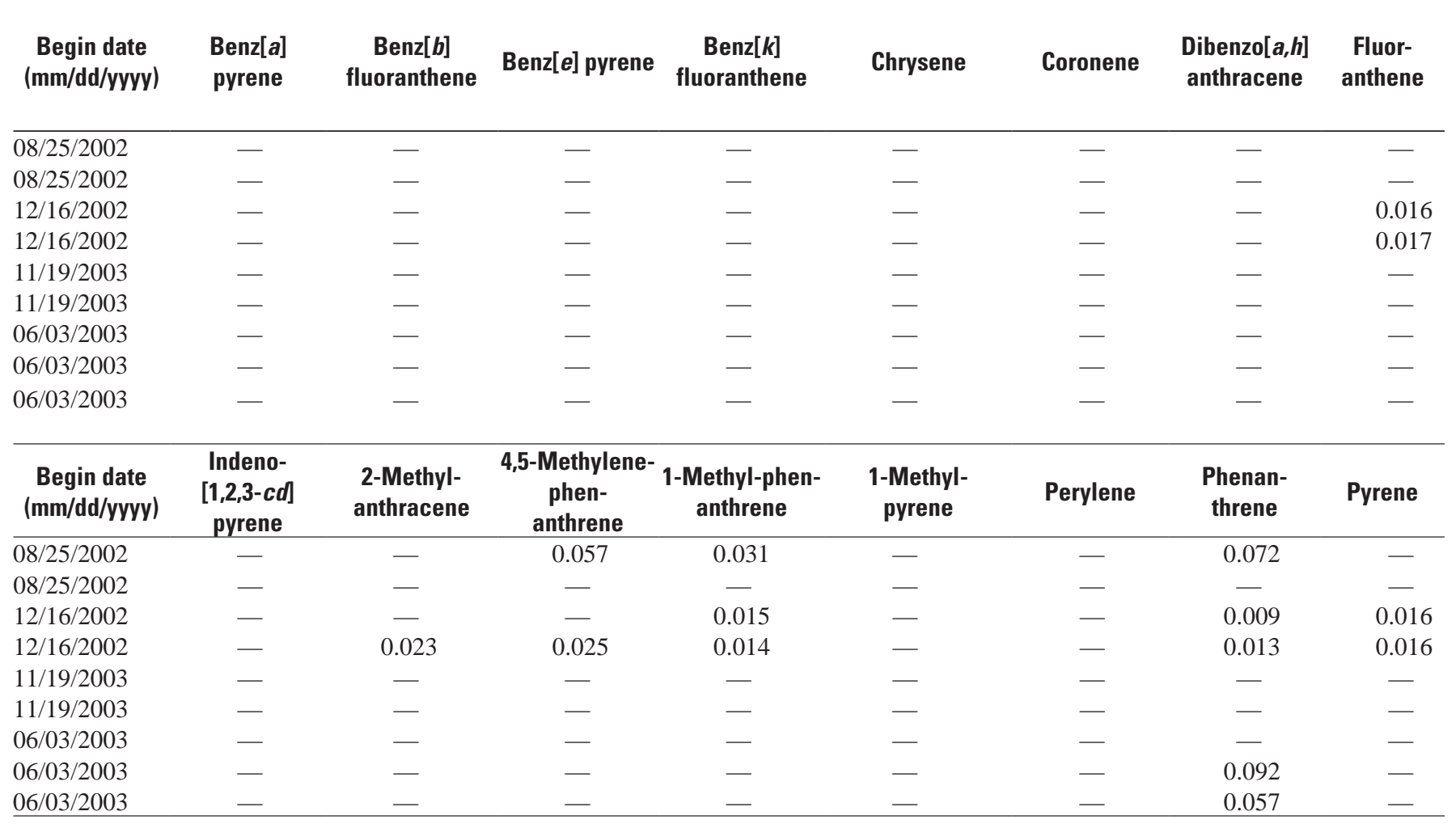


Table 23A. Quality-control results for laboratory and field reagent blanks and laboratory solvent blanks for polycyclic aromatic hydrocarbon (PAH) compound concentrations from the Sweetwater Reservoir atmospheric site, San Diego County, California.Continued

[All blank analyses by gas chromatography/mass spectrometry with selected ion monitoring (SIM). Field blank identification number is 324141117001601 . Concentrations are given in nanograms per cubic meter $\left(\mathrm{ng} / \mathrm{m}^{3}\right)$ assuming a $315-\mathrm{m}^{3}$ sample volume, unless noted. GFF, glass fiber filter; PSE, pressurized solvent extraction; PUF, polyurethane foam plug; —, compound not detected at a concentration above laboratory reporting level]

\begin{tabular}{|c|c|c|c|c|c|c|c|c|}
\hline $\begin{array}{l}\text { Begin date } \\
\text { (mm/dd/yyyy) }\end{array}$ & $\begin{array}{c}\text { C1-178 } \\
\text { Isomers, } \\
\text { methylated } \\
\text { phen- } \\
\text { anthrenes/ } \\
\text { anthracenes }\end{array}$ & $\begin{array}{c}\text { C1-202 } \\
\text { Isomers, } \\
\text { methylated } \\
\text { fluoranthenes/ } \\
\text { pyrenes }\end{array}$ & $\begin{array}{c}\text { C1-228 } \\
\text { Isomers, } \\
\text { methylated } \\
\text { benz[a] } \\
\text { anthracenes/ } \\
\text { chrysenes }\end{array}$ & $\begin{array}{c}\text { C1-252 Isomers, } \\
\text { methylated } \\
\text { benz- } \\
\text { pyrenes/ } \\
\text { perylenes }\end{array}$ & $\begin{array}{c}\text { C2-178 } \\
\text { Isomers, } \\
\text { alkylated } \\
\text { phenanthrenes/ } \\
\text { anthracenes }\end{array}$ & $\begin{array}{c}\text { C2-202 } \\
\text { Isomers, } \\
\text { alkylated } \\
\text { fluoranthenes/ } \\
\text { pyrenes }\end{array}$ & $\begin{array}{c}\text { C2-228 } \\
\text { Isomers, } \\
\text { alkylated } \\
\text { benz[a]- } \\
\text { anthracenes/ } \\
\text { chrysenes }\end{array}$ & $\begin{array}{c}\text { C2-252 } \\
\text { Isomers, } \\
\text { alkylated } \\
\text { benz- } \\
\text { pyrenes/ } \\
\text { perylenes }\end{array}$ \\
\hline
\end{tabular}

\begin{tabular}{lcl}
\hline $08 / 25 / 2002$ & 0.104 & - \\
$08 / 25 / 2002$ & - & - \\
$12 / 16 / 2002$ & - & - \\
$12 / 16 / 2002$ & 0.034 & - \\
$11 / 19 / 2003$ & - & - \\
$11 / 19 / 2003$ & - & - \\
$06 / 03 / 2003$ & - & - \\
$06 / 03 / 2003$ & - & - \\
$06 / 03 / 2003$ & - & -
\end{tabular}

\begin{tabular}{|c|c|c|c|c|c|c|c|}
\hline $\begin{array}{l}\text { Begin date } \\
\text { (mm/dd/yyyy) }\end{array}$ & $\begin{array}{c}\text { C3-178 } \\
\text { Isomers, } \\
\text { alkylated } \\
\text { phen- } \\
\text { anthrenes/ } \\
\text { anthracenes }\end{array}$ & $\begin{array}{l}\text { C3-202 Isomers, } \\
\text { alkylated } \\
\text { fluoranthenes/ } \\
\text { pyrenes }\end{array}$ & $\begin{array}{c}\text { C3-228 I } \\
\text { somers, } \\
\text { alkylated } \\
\text { benz[a] } \\
\text { anthracenes/ } \\
\text { chrysenes } \\
\end{array}$ & $\begin{array}{c}\text { C3-252 Isomers, } \\
\text { alkylated benz- } \\
\text { pyrenes/ } \\
\text { perylenes }\end{array}$ & $\begin{array}{c}\text { C4-178 } \\
\text { Isomers, } \\
\text { alkylated } \\
\text { phenanthrenes/ } \\
\text { anthracenes }\end{array}$ & $\begin{array}{c}\text { C4-202 } \\
\text { Isomers, } \\
\text { alkylated } \\
\text { fluoranthenes/ } \\
\text { pyrenes } \\
\end{array}$ & $\begin{array}{c}\text { C4-228 } \\
\text { Isomers, } \\
\text { alkylated } \\
\text { benz[a] } \\
\text { anthracenes/ } \\
\text { chrysenes } \\
\end{array}$ \\
\hline $08 / 25 / 2002$ & - & - & - & - & - & - & - \\
\hline 08/25/2002 & - & - & - & - & - & - & - \\
\hline $12 / 16 / 2002$ & - & - & - & - & - & - & - \\
\hline $12 / 16 / 2002$ & - & - & - & - & - & - & - \\
\hline $11 / 19 / 2003$ & - & - & - & - & - & - & - \\
\hline $11 / 19 / 2003$ & - & - & - & - & - & - & - \\
\hline 06/03/2003 & - & - & - & - & - & - & - \\
\hline 06/03/2003 & - & - & - & - & - & - & - \\
\hline $06 / 03 / 2003$ & - & - & - & - & - & - & - \\
\hline $\begin{array}{l}\text { Begin date } \\
\text { (mm/dd/yyyy) }\end{array}$ & $\begin{array}{c}\text { C5-178 } \\
\text { Isomers, } \\
\text { alkylated } \\
\text { phen- } \\
\text { anthrenes/ } \\
\text { anthracenes }\end{array}$ & $\begin{array}{l}\text { C5-202 Isomers, } \\
\text { alkylated } \\
\text { fluoranthenes/ } \\
\text { pyrenes }\end{array}$ & $\begin{array}{c}\text { C5-228 } \\
\text { Isomers, } \\
\text { alkylated } \\
\text { benz[a] } \\
\text { anthracenes/ } \\
\text { chrysenes } \\
\end{array}$ & $\begin{array}{c}\text { C5-252 } \\
\text { Isomers, } \\
\text { alkylated } \\
\text { benz-pyrenes/ } \\
\text { perylenes }\end{array}$ & $\begin{array}{l}\text { 2-Fluoro-biphenyl, } \\
\text { surrogate } \\
\text { (percent) }\end{array}$ & $\begin{array}{c}\text { Nitro- } \\
\text { benzene- } d 5, \\
\text { surrogate } \\
\text { (percent) }\end{array}$ & $\begin{array}{l}\text { Terphenyl-d14, } \\
\text { surrogate } \\
\text { (percent) }\end{array}$ \\
\hline $08 / 25 / 2002$ & - & - & - & - & 89.1 & 61.6 & 117 \\
\hline $08 / 25 / 2002$ & - & - & - & - & 32.6 & 20.4 & 117 \\
\hline $12 / 16 / 2002$ & - & - & - & - & 33.0 & 23.0 & 76.7 \\
\hline $12 / 16 / 2002$ & - & - & - & - & 68.0 & 62.3 & 83.1 \\
\hline $11 / 19 / 2003$ & - & - & - & - & 29.4 & 24.2 & 83.8 \\
\hline $11 / 19 / 2003$ & - & - & - & - & 94.6 & 95.8 & 103 \\
\hline 06/03/2003 & - & - & - & - & 77.6 & 68.2 & 89.2 \\
\hline 06/03/2003 & - & - & - & - & 87.2 & 90.2 & 96.8 \\
\hline $06 / 03 / 2003$ & - & - & - & - & 88.8 & 86.4 & 98.6 \\
\hline
\end{tabular}


Table 23B. Quality-control results for polycyclic aromatic hydrocarbon (PAH) compound laboratory reagent-spikes for air samples collected at the Sweetwater Reservoir atmospheric site, San Diego County, California.

[All laboratory spike samples were analyzed using gas chromatography/mass spectrometry with selected ion monitoring (SIM). Alkylated PAHs were not spiked, so are not listed in this table. Values for compounds are in percent recovery. PUF, polyurethane foam; GFF, glass fiber filter; PSE, pressurized solvent extraction]

\begin{tabular}{|c|c|c|c|c|c|c|c|c|}
\hline $\begin{array}{l}\text { Begin date } \\
\text { (mm/dd/yyyy) }\end{array}$ & $\begin{array}{c}\text { Sample } \\
\text { component } \\
\text { spiked }\end{array}$ & $\begin{array}{c}\text { Sample } \\
\text { preparation } \\
\text { set number }\end{array}$ & $\begin{array}{l}\text { Extraction } \\
\text { by }\end{array}$ & $\begin{array}{l}\text { Extract } \\
\text { split }\end{array}$ & Anthracene & $\begin{array}{c}\text { Benz }[a] \\
\text { anthracene }\end{array}$ & $\begin{array}{l}\text { Benz[ghi] } \\
\text { perylene }\end{array}$ & $\begin{array}{l}\text { Benz[a] } \\
\text { pyrene }\end{array}$ \\
\hline $8 / 25 / 2002$ & PUF & 02.237 & Soxhlet & No & 100 & 116 & 96.7 & 114 \\
\hline $8 / 25 / 2002$ & GFF & 02.237 & Soxhlet & No & 60.0 & 98.8 & 98.0 & 98.0 \\
\hline $8 / 25 / 2002$ & $\begin{array}{l}\text { Solvent only (no } \\
\text { matrix) }\end{array}$ & 02.237 & Soxhlet & No & 54.6 & 93.4 & 98.3 & 88.5 \\
\hline $12 / 16 / 2002$ & PUF & 02.35009 & PSE & No & 68.3 & 71.5 & 59.8 & 71.2 \\
\hline $11 / 19 / 2003$ & GFF & 03.32308 & PSE & Yes & 68.7 & 67.5 & 84.7 & 68.7 \\
\hline $11 / 19 / 2003$ & PUF & 03.32308 & PSE & Yes & 85.0 & 85.3 & 91.3 & 85.2 \\
\hline $8 / 25 / 2002$ & 74.0 & 120 & 96.0 & 120 & 124 & 104 & 91.7 & 110 \\
\hline $8 / 25 / 2002$ & 85.3 & 119 & 93.9 & 118 & 125 & 87.0 & 85.0 & 69.9 \\
\hline $8 / 25 / 2002$ & 87.0 & 125 & 93.3 & 116 & 124 & 87.4 & 65.8 & 63.8 \\
\hline $12 / 16 / 2002$ & 82.1 & 84.9 & 68.1 & 77.5 & 81.1 & 70.0 & 75.8 & 68.2 \\
\hline $12 / 16 / 2002$ & 62.5 & 66.5 & 66.7 & 51.2 & 64.1 & 71.7 & 63.6 & 75.5 \\
\hline $11 / 19 / 2003$ & 97.0 & 91.5 & 81.7 & 86.0 & 73.8 & 78.2 & 62.3 & 65.3 \\
\hline $11 / 19 / 2003$ & 95.7 & 101 & 92.0 & 87.2 & 86.7 & 96.8 & 80.2 & 85.0 \\
\hline
\end{tabular}

\begin{tabular}{|c|c|c|c|c|c|c|c|c|}
\hline $\begin{array}{l}\text { Begin date } \\
\text { (mm/dd/yyyy) }\end{array}$ & $\begin{array}{c}\text { 1-Methyl- } \\
\text { phenanthrene }\end{array}$ & $\begin{array}{l}\text { 1-Methyl- } \\
\text { pyrene }\end{array}$ & Perylene & Phenanthrene & Pyrene & $\begin{array}{l}\text { 2-Fluoro- } \\
\text { biphenyl, } \\
\text { surrogate } \\
\text { (percent) }\end{array}$ & $\begin{array}{c}\text { Nitro- } \\
\text { benzene- } d 5 \text {, } \\
\text { surrogate } \\
\text { (percent) }\end{array}$ & $\begin{array}{l}\text { Terphenyl-d14, } \\
\text { surrogate } \\
\text { (percent) }\end{array}$ \\
\hline $8 / 25 / 2002$ & 93.6 & 107 & 98.5 & 95.5 & 99.3 & 97.2 & 82.4 & 117 \\
\hline $8 / 25 / 2002$ & 73.1 & 100 & 83.4 & 65.0 & 86.1 & 60.5 & 38.8 & 111 \\
\hline $12 / 16 / 2002$ & 66.4 & 69.7 & 63.4 & 63.6 & 69.0 & 55.7 & 51.5 & 75.5 \\
\hline $12 / 16 / 2002$ & 66.3 & 74.0 & 62.2 & 64.6 & 71.4 & 54.2 & 46.5 & 79.4 \\
\hline $11 / 19 / 2003$ & 80.8 & 76.8 & 78.5 & 79.0 & 79.3 & 73.8 & 99.8 & 86.2 \\
\hline $11 / 19 / 2003$ & 91.8 & 96.3 & 84.7 & 93.3 & 96.8 & 97.8 & 108 & 99.6 \\
\hline
\end{tabular}


Table 24A. Quality-control results for pesticide blanks using U.S. Geological Survey's National Water Quality Laboratory method Airpest001 for air samples collected at the Sweetwater Reservoir atmospheric site, San Diego County, California.

[Concentrations are given in nanograms per cubic meter unless noted. - , compound not detected at a concentration above laboratory reporting level. A sample volume of 315 cubic meters was assumed]

\begin{tabular}{|c|c|c|c|c|c|c|c|}
\hline Begin date & $\begin{array}{c}\text { QC } \\
\text { sample } \\
\text { type }\end{array}$ & $\begin{array}{c}\text { Sample } \\
\text { component } \\
\text { used }\end{array}$ & $\begin{array}{c}\text { Sample } \\
\text { preparation } \\
\text { set number }\end{array}$ & Acetochlor & Alachlor & Atrazine & $\begin{array}{c}\text { Azinphos- } \\
\text { methyl } \\
\text { (estimated) }\end{array}$ \\
\hline $8 / 25 / 2002$ & $\begin{array}{l}\text { Laboratory PUF } \\
\text { blank }\end{array}$ & $\begin{array}{l}1 \text { clean used field } \\
\text { PUF }\end{array}$ & 02.037 & - & - & - & - \\
\hline $8 / 25 / 2002$ & Solvent Blank & Solvent only & 02.037 & - & - & - & - \\
\hline Begin date & $\begin{array}{l}\text { Benfluralin } \\
\text { (estimated) }\end{array}$ & $\begin{array}{c}\text { Carbaryl } \\
\text { (estimated) }\end{array}$ & $\begin{array}{l}\text { Carbofuran } \\
\text { (estimated) }\end{array}$ & Chlorpyrifos & Cyanazine & $\begin{array}{l}\text { Dacthal } \\
\text { (DCPA) }\end{array}$ & $p, p^{\prime}$ DDE \\
\hline $8 / 25 / 2002$ & - & - & - & - & - & 0.036 & 0.042 \\
\hline $8 / 25 / 2002$ & - & - & - & - & - & - & - \\
\hline Begin date & Deethylatrazine & Diazinon & Dieldrin & $\begin{array}{l}\text { Disulfoton } \\
\text { (estimated) }\end{array}$ & $\begin{array}{l}\text { Ethalfluralin } \\
\text { (estimated) }\end{array}$ & Ethion & Ethoprophos \\
\hline $8 / 25 / 2002$ & - & - & - & - & - & - & - \\
\hline $8 / 25 / 2002$ & - & - & - & - & - & - & - \\
\hline Begin date & Fonofos & $\alpha-\mathrm{HCH}$ & Lindane & Linuron & Malathion & Metolachlor & Metribuzin \\
\hline $8 / 25 / 2002$ & - & - & - & - & - & - & - \\
\hline $8 / 25 / 2002$ & - & - & - & - & - & - & - \\
\hline Begin date & $\begin{array}{c}\text { Molinate } \\
\text { (estimated) }\end{array}$ & Napropamide & Parathion & $\begin{array}{l}\text { Parathion- } \\
\text { methyl }\end{array}$ & $\begin{array}{c}\text { Pendi- } \\
\text { methalin } \\
\text { (estimated) }\end{array}$ & $\begin{array}{c}\text { cis- } \\
\text { Permethrin }\end{array}$ & $\begin{array}{c}\text { Phorate } \\
\text { (estimated) }\end{array}$ \\
\hline $8 / 25 / 2002$ & - & - & - & - & - & - & - \\
\hline $8 / 25 / 2002$ & - & - & - & - & - & - & - \\
\hline Begin date & $\begin{array}{l}\text { Prometon } \\
\text { (estimated) }\end{array}$ & Propyzamide & Propachlor & Propanil & $\begin{array}{l}\text { Propargite } \\
\text { I \& II }\end{array}$ & Simazine & $\begin{array}{l}\text { Tebuthiuron } \\
\text { (estimated) }\end{array}$ \\
\hline $8 / 25 / 2002$ & - & - & - & - & - & - & - \\
\hline $8 / 25 / 2002$ & - & - & - & - & - & - & - \\
\hline Begin date & $\begin{array}{l}\text { Terbufos } \\
\text { (estimated) }\end{array}$ & Thiobencarb & Triallate & Trifluralin & $\begin{array}{c}\text { Diazinon-d10, } \\
\text { surrogate } \\
\text { (percent) }\end{array}$ & $\begin{array}{c}\alpha-H C H-d 6, \\
\text { surrogate } \\
\text { (percent) }\end{array}$ & \\
\hline $8 / 25 / 2002$ & - & - & - & E0.040 & 98.0 & 84.4 & \\
\hline $8 / 25 / 2002$ & - & - & - & - & 55.0 & 40.0 & \\
\hline
\end{tabular}


Table 24A. Quality-control results for pesticide blanks using U.S. Geological Survey's National Water Quality Laboratory method Airpest001 for air samples collected at the Sweetwater Reservoir atmospheric site, San Diego County, California.Continued

[Concentrations are given in nanograms per cubic meter unless noted. - , compound not detected at a concentration above laboratory reporting level. A sample volume of 315 cubic meters was assumed]

\begin{tabular}{|c|c|c|c|c|c|c|c|}
\hline Begin date & $\begin{array}{c}\text { QC } \\
\text { sample } \\
\text { type }\end{array}$ & $\begin{array}{c}\text { Sample } \\
\text { component } \\
\text { used }\end{array}$ & $\begin{array}{c}\text { Sample } \\
\text { preparation } \\
\text { set number }\end{array}$ & Acetochlor & Alachlor & Atrazine & $\begin{array}{l}\text { Azinphos- } \\
\text { methyl } \\
\text { (estimated) }\end{array}$ \\
\hline $8 / 25 / 2002$ & $\begin{array}{l}\text { Laboratory PUF } \\
\text { blank }\end{array}$ & $\begin{array}{l}1 \text { clean used field } \\
\text { PUF }\end{array}$ & 02.037 & - & - & - & - \\
\hline $8 / 25 / 2002$ & Solvent Blank & Solvent only & 02.037 & - & - & - & - \\
\hline Begin date & $\begin{array}{l}\text { Benfluralin } \\
\text { (estimated) }\end{array}$ & $\begin{array}{c}\text { Carbaryl } \\
\text { (estimated) }\end{array}$ & $\begin{array}{l}\text { Carbofuran } \\
\text { (estimated) }\end{array}$ & Chlorpyrifos & Cyanazine & $\begin{array}{l}\text { Dacthal } \\
\text { (DCPA) }\end{array}$ & $p, p^{\prime}$ DDE \\
\hline $8 / 25 / 2002$ & - & - & - & - & - & 0.036 & 0.042 \\
\hline $8 / 25 / 2002$ & - & - & - & - & - & - & - \\
\hline Begin date & Deethylatrazine & Diazinon & Dieldrin & $\begin{array}{l}\text { Disulfoton } \\
\text { (estimated) }\end{array}$ & $\begin{array}{l}\text { Ethalfluralin } \\
\text { (estimated) }\end{array}$ & Ethion & Ethoprophos \\
\hline $8 / 25 / 2002$ & - & - & - & - & - & - & - \\
\hline $8 / 25 / 2002$ & - & - & - & - & - & - & - \\
\hline Begin date & Fonofos & $\alpha-\mathrm{HCH}$ & Lindane & Linuron & Malathion & Metolachlor & Metribuzin \\
\hline $8 / 25 / 2002$ & - & - & - & - & - & - & - \\
\hline $8 / 25 / 2002$ & - & - & - & - & - & - & - \\
\hline Begin date & $\begin{array}{c}\text { Molinate } \\
\text { (estimated) }\end{array}$ & Napropamide & Parathion & $\begin{array}{l}\text { Parathion- } \\
\text { methyl }\end{array}$ & $\begin{array}{c}\text { Pendi- } \\
\text { methalin } \\
\text { (estimated) }\end{array}$ & $\begin{array}{c}\text { cis- } \\
\text { Permethrin }\end{array}$ & $\begin{array}{c}\text { Phorate } \\
\text { (estimated) }\end{array}$ \\
\hline $8 / 25 / 2002$ & - & - & - & - & - & - & - \\
\hline $8 / 25 / 2002$ & - & - & - & - & - & - & - \\
\hline Begin date & $\begin{array}{l}\text { Prometon } \\
\text { (estimated) }\end{array}$ & Propyzamide & Propachlor & Propanil & $\begin{array}{l}\text { Propargite } \\
\text { I \& II }\end{array}$ & Simazine & $\begin{array}{l}\text { Tebuthiuron } \\
\text { (estimated) }\end{array}$ \\
\hline $8 / 25 / 2002$ & - & - & - & - & - & - & - \\
\hline $8 / 25 / 2002$ & - & - & - & - & - & - & - \\
\hline Begin date & $\begin{array}{c}\text { Terbufos } \\
\text { (estimated) }\end{array}$ & Thiobencarb & Triallate & Trifluralin & $\begin{array}{l}\text { Diazinon-d10, } \\
\text { surrogate } \\
\text { (percent) }\end{array}$ & $\begin{array}{c}\alpha-\mathrm{HCH}-\alpha 6, \\
\text { surrogate } \\
\text { (percent) }\end{array}$ & \\
\hline $8 / 25 / 2002$ & - & - & - & E0.040 & 98.0 & 84.4 & \\
\hline $8 / 25 / 2002$ & - & - & - & - & 55.0 & 40.0 & \\
\hline
\end{tabular}


Table 24B. Quality-control results for pesticide laboratory reagent-spikes using U.S. Geological Survey's National Water Quality Laboratory method Airpest001 for air samples collected at the Sweetwater Reservoir atmospheric site, San Diego County, California.

[Concentrations are given in nanograms per cubic meter unless noted. A sample volume of 315 cubic meters was assumed]

\begin{tabular}{|c|c|c|c|c|c|c|c|}
\hline Begin date & $\begin{array}{c}\text { QC } \\
\text { sample } \\
\text { type }\end{array}$ & $\begin{array}{c}\text { Sample } \\
\text { component } \\
\text { used }\end{array}$ & $\begin{array}{c}\text { Sample } \\
\text { preparation } \\
\text { set number }\end{array}$ & Acetochlor & Alachlor & Atrazine & $\begin{array}{c}\text { Azinphos- } \\
\text { methyl } \\
\text { (estimated) }\end{array}$ \\
\hline $8 / 25 / 2002$ & $\begin{array}{l}\text { Laboratory PUF } \\
\text { blank }\end{array}$ & $\begin{array}{l}1 \text { clean used field } \\
\text { PUF }\end{array}$ & 02.037 & 97.2 & 105 & 91.9 & 121 \\
\hline $8 / 25 / 2002$ & Solvent Blank & Solvent only & 02.037 & 66.5 & 74.2 & 81.3 & 97.5 \\
\hline Begin date & $\begin{array}{l}\text { Benfluralin } \\
\text { (estimated) }\end{array}$ & $\begin{array}{c}\text { Carbaryl } \\
\text { (estimated) }\end{array}$ & $\begin{array}{l}\text { Carbofuran } \\
\text { (estimated) }\end{array}$ & Chlorpyrifos & Cyanazine & $\begin{array}{l}\text { Dacthal } \\
\text { (DCPA) }\end{array}$ & $p, p^{\prime} \mathrm{DDE}$ \\
\hline $8 / 25 / 2002$ & 71.8 & 108 & 102 & 79.0 & 74.9 & 107 & 112 \\
\hline $8 / 25 / 2002$ & 19.2 & 67.9 & 132 & 72.6 & 73.0 & 77.9 & 88.1 \\
\hline
\end{tabular}

\begin{tabular}{|c|c|c|c|c|c|c|c|}
\hline Begin date & Deethylatrazine & Diazinon & Dieldrin & $\begin{array}{l}\text { Disulfoton } \\
\text { (estimated) }\end{array}$ & $\begin{array}{l}\text { Ethalfluralin } \\
\text { (estimated) }\end{array}$ & Ethion & Ethoprophos \\
\hline $8 / 25 / 2002$ & 95.8 & 89.7 & 105 & 55.0 & 66.7 & 96.2 & 78.0 \\
\hline $8 / 25 / 2002$ & 86.5 & 70.2 & 86.3 & 8.5 & 12.9 & 87.0 & 59.5 \\
\hline Begin date & Fonofos & $\alpha-\mathrm{HCH}$ & Lindane & Linuron & Malathion & Metolachlor & Metribuzin \\
\hline $8 / 25 / 2002$ & 88.6 & 84.4 & 94.8 & 119 & 91.4 & 95.2 & 81.1 \\
\hline $8 / 25 / 2002$ & 69.8 & 61.2 & 60.3 & 117 & 83.8 & 79.8 & 72.0 \\
\hline
\end{tabular}

\begin{tabular}{|c|c|c|c|c|c|c|c|}
\hline Begin date & $\begin{array}{c}\text { Molinate } \\
\text { (estimated) }\end{array}$ & Napropamide & Parathion & $\begin{array}{l}\text { Parathion- } \\
\text { methyl }\end{array}$ & $\begin{array}{l}\text { Pendimethalin } \\
\text { (estimated) }\end{array}$ & cis-Permethrin & $\begin{array}{c}\text { Phorate } \\
\text { (estimated) }\end{array}$ \\
\hline $8 / 25 / 2002$ & 71.2 & 113 & 80.0 & 97.7 & 85.9 & 102 & 77.9 \\
\hline $8 / 25 / 2002$ & 52.6 & 81.6 & 50.2 & 47.9 & 38.2 & 90.2 & 34.5 \\
\hline Begin date & $\begin{array}{l}\text { Prometon } \\
\text { (estimated) }\end{array}$ & Propyzamide & Propachlor & Propanil & $\begin{array}{l}\text { Propargite } \\
\text { I \& II }\end{array}$ & Simazine & $\begin{array}{l}\text { Tebuthiuron } \\
\text { (estimated) }\end{array}$ \\
\hline $8 / 25 / 2002$ & 87.5 & 90.3 & 89.0 & 104 & 93.0 & 66.2 & 65.5 \\
\hline $8 / 25 / 2002$ & 76.9 & 74.3 & 67.3 & 89.8 & 79.3 & 54.1 & 62.3 \\
\hline Begin date & $\begin{array}{c}\text { Terbufos } \\
\text { (estimated) }\end{array}$ & Thiobencarb & Triallate & $\begin{array}{l}\text { Trifluralin } \\
\text { (estimated) }\end{array}$ & $\begin{array}{c}\text { Diazinon-d10, } \\
\text { surrogate } \\
\text { (percent) }\end{array}$ & $\begin{array}{l}\alpha-\mathrm{HCH}-\alpha 6, \\
\text { surrogate } \\
\text { (percent) }\end{array}$ & \\
\hline $8 / 25 / 2002$ & 68.4 & 122 & 87.9 & 80.6 & 93.5 & 79.8 & \\
\hline $8 / 25 / 2002$ & 39.5 & 78.0 & 71.6 & 24.3 & 83.0 & 56.4 & \\
\hline
\end{tabular}


Table 25A. Quality-control laboratory blank results using pesticide method Airpest002 for air samples collected at the Sweetwater Reservoir atmospheric site, San Diego County, California.

[Concentrations are given in nanograms per cubic meter unless noted. Sample volume was assumed to be 315 cubic meters. QC, quality control; -, compound not detected at a concentration above laboratory reporting level]

\begin{tabular}{|c|c|c|c|c|c|c|c|c|}
\hline $\begin{array}{c}\text { OC } \\
\text { sample } \\
\text { type }\end{array}$ & $\begin{array}{c}\text { Sample } \\
\text { component used }\end{array}$ & $\begin{array}{c}\text { Sample } \\
\text { preparation set } \\
\text { number }\end{array}$ & $\begin{array}{l}\text { 2-Amino- } N \text { - } \\
\text { isopropyl- } \\
\text { benzamide }\end{array}$ & $\begin{array}{c}\text { Azinphos-methyl } \\
\text { oxon } \\
\text { (estimated) }\end{array}$ & Bifenthrin & $\begin{array}{c}\text { 2-(4-tert- } \\
\text { Butylphenoxy) } \\
\text { cyclo- } \\
\text { hexanol }\end{array}$ & $\begin{array}{c}\text { 4-Chlorobenzyl- } \\
\text { methyl- } \\
\text { sulfone }\end{array}$ & $\begin{array}{c}\text { 2-Chloro-2',6'- } \\
\text { diethyl- } \\
\text { acetanilide }\end{array}$ \\
\hline Laboratory blank & $\begin{array}{l}\text { Clean, used field } \\
\text { PUF }\end{array}$ & 02.037 & - & - & - & - & - & - \\
\hline $\begin{array}{l}\text { Laboratory solvent } \\
\text { blank }\end{array}$ & Solvent only & 02.037 & - & - & - & - & - & - \\
\hline $\begin{array}{c}\text { OC sample } \\
\text { type }\end{array}$ & $\begin{array}{c}\text { 4-Chloro-2- } \\
\text { methylphenol } \\
\text { (estimated) }\end{array}$ & Cycloate & $\lambda$-Cyhalothrin & $\begin{array}{l}\text { Cyfluthrin } \\
\text { (estimated) }\end{array}$ & $\begin{array}{l}\text { Cypermethrin } \\
\text { (estimated) }\end{array}$ & $\begin{array}{c}\text { 2,5-Di- } \\
\text { chloroaniline }\end{array}$ & $\begin{array}{c}\text { 3,4-Di- } \\
\text { chloroaniline } \\
\text { (estimated) }\end{array}$ & $\begin{array}{c}\text { 3,5-Di- } \\
\text { chloroaniline }\end{array}$ \\
\hline Laboratory blank & - & - & - & - & - & - & - & - \\
\hline $\begin{array}{l}\text { Laboratory solvent } \\
\text { blank }\end{array}$ & - & - & - & - & - & - & - & - \\
\hline $\begin{array}{c}\text { OC sample } \\
\text { type }\end{array}$ & $\begin{array}{l}\text { 4,4'-Dichloro- } \\
\text { benzophenone }\end{array}$ & Dimethoate & E-Dimethomorph & Z-Dimethomorph & $\begin{array}{c}\text { Disulfoton } \\
\text { sulfone }\end{array}$ & $\alpha$-Endosulfan & $\beta$-Endosulfan & Endosulfan ether \\
\hline Laboratory blank & - & - & - & - & - & - & - & - \\
\hline $\begin{array}{l}\text { Laboratory solvent } \\
\text { blank }\end{array}$ & - & - & - & - & - & - & - & - \\
\hline $\begin{array}{l}\text { OC sample } \\
\text { type }\end{array}$ & Endosulfan sulfate & Ethion & Ethion monoxon & Ethoprophos & $\begin{array}{c}\text { 2-Ethyl-6- } \\
\text { methylaniline } \\
\text { (estimated) }\end{array}$ & Fenthion & Fenthion sulfone & $\begin{array}{c}\text { Fenthion } \\
\text { sulfone } \\
\text { oxygen analog } \\
\end{array}$ \\
\hline Laboratory blank & - & - & - & - & - & - & - & - \\
\hline $\begin{array}{l}\text { Laboratory solvent } \\
\text { blank }\end{array}$ & - & - & - & - & - & - & - & - \\
\hline $\begin{array}{l}\text { OC sample } \\
\text { type }\end{array}$ & $\begin{array}{l}\text { Fenthion } \\
\text { sulfoxide }\end{array}$ & $\begin{array}{l}\text { Flumetralin } \\
\text { (estimated) }\end{array}$ & $\begin{array}{c}\text { Fonofos } \\
\text { oxygen analog } \\
\text { (estimated) }\end{array}$ & $\begin{array}{l}\text { Iprodione } \\
\text { (estimated) }\end{array}$ & Isofenphos & Malaoxon & Methidathion & $\begin{array}{l}\text { 1,4-Naphtho- } \\
\text { quinone } \\
\text { (estimated) }\end{array}$ \\
\hline Laboratory blank & - & - & - & - & - & - & - & - \\
\hline $\begin{array}{l}\text { Laboratory solvent } \\
\text { blank }\end{array}$ & - & - & - & - & - & - & - & - \\
\hline
\end{tabular}

\begin{tabular}{|c|c|c|c|c|c|c|c|c|}
\hline $\begin{array}{l}\text { QC sample } \\
\text { type }\end{array}$ & Myclobutanil & Oxyfluorfen & Paraoxon-ethyl & $\begin{array}{c}\text { Paraoxon- } \\
\text { methyl } \\
\text { (estimated) }\end{array}$ & Profenofos & Prometryn & Propetamphos & $\begin{array}{l}\text { cis-Propi- } \\
\text { conazole }\end{array}$ \\
\hline Laboratory blank & - & - & - & - & - & - & - & - \\
\hline $\begin{array}{l}\text { Laboratory solvent } \\
\text { blank }\end{array}$ & - & - & - & - & - & - & - & - \\
\hline
\end{tabular}


Table 25A. Quality-control laboratory blank results using pesticide method Airpest002 for air samples collected at the Sweetwater Reservoir atmospheric site, San Diego County, California.-Continued

[Concentrations are given in nanograms per cubic meter unless noted. Sample volume was assumed to be 315 cubic meters. QC, quality control; -, compound not detected at a concentration above laboratory reporting level]

\begin{tabular}{|c|c|c|c|c|c|c|c|c|}
\hline $\begin{array}{l}\text { OC sample } \\
\text { type }\end{array}$ & $\begin{array}{c}\text { trans- } \\
\text { Propiconazole }\end{array}$ & $\begin{array}{c}\text { O-ethyl-0- } \\
\text { methyl-S- } \\
\text { Propyl- } \\
\text { phosphorthioate }\end{array}$ & Sulfotepp & Tebupirimphos & $\begin{array}{l}\text { Tebupirimphos } \\
\text { oxygen analog }\end{array}$ & Tefluthrin & $\begin{array}{l}\text { Temephos } \\
\text { (estimated) }\end{array}$ & $\begin{array}{l}\text { Terbufos oxygen } \\
\text { analog sulfone }\end{array}$ \\
\hline $\begin{array}{l}\text { Laboratory solvent } \\
\text { blank }\end{array}$ & - & - & - & - & - & - & - & - \\
\hline Laboratory blank & - & - & - & 102 & 83.4 & & & \\
\hline $\begin{array}{l}\text { Laboratory solvent } \\
\text { blank }\end{array}$ & - & - & - & 50.8 & 47.2 & & & \\
\hline
\end{tabular}


Table 25B. Quality-control results for pesticide laboratory reagent spikes using method Airpest002 for samples collected at the Sweetwater Reservoir atmospheric site, San Diego County, California.

[The site identification number is 324141117001601. Concentrations are given in percent recovered. PUF, polyurethane foam plug; NS, not spiked]

\begin{tabular}{|c|c|c|c|c|c|c|c|}
\hline $\begin{array}{c}\text { Sample } \\
\text { component } \\
\text { used }\end{array}$ & $\begin{array}{c}\text { Sample } \\
\text { preparation } \\
\text { set number }\end{array}$ & $\begin{array}{l}\text { 2-Amino- } N \text { - } \\
\text { isopropyl- } \\
\text { benzamide }\end{array}$ & $\begin{array}{l}\text { Azinphos- } \\
\text { methyl oxon } \\
\text { (estimated) }\end{array}$ & Bifenthrin & $\begin{array}{c}\text { 2-(4-tert- } \\
\text { Butylphenoxy) } \\
\text { cyclohexanol }\end{array}$ & $\begin{array}{c}\text { 4-Chloro- } \\
\text { benzylmethyl } \\
\text { sulfone }\end{array}$ & $\begin{array}{c}\text { 2-Chloro-2',6'- } \\
\text { diethyl- } \\
\text { acetanilide }\end{array}$ \\
\hline $\begin{array}{l}1 \text { clean used field } \\
\text { PUF }\end{array}$ & 02.037 & 51.0 & 85.6 & 106 & 97.4 & NS & 101 \\
\hline Solvent only & 02.037 & 43.5 & 53.4 & 78.4 & 59.7 & NS & 72.4 \\
\hline Solvent only & 02.037 & 26.4 & 0.00 & 72.5 & 90.2 & NS & 67.1 \\
\hline $\begin{array}{c}\text { Sample } \\
\text { component } \\
\text { used }\end{array}$ & $\begin{array}{c}\text { 4-Chloro-2- } \\
\text { methylphenol } \\
\text { (estimated) }\end{array}$ & Cycloate & $\lambda$-Cyhalothrin & $\begin{array}{l}\text { Cyfluthrin } \\
\text { (estimated) }\end{array}$ & $\begin{array}{c}\text { Cypermethrin } \\
\text { (estimated) }\end{array}$ & $\begin{array}{l}\text { 2,5-Dichloro- } \\
\text { aniline }\end{array}$ & $\begin{array}{l}\text { 3,4-Dichloro- } \\
\text { aniline } \\
\text { (estimated) }\end{array}$ \\
\hline $\begin{array}{l}1 \text { clean used field } \\
\text { PUF }\end{array}$ & 87.7 & 84.7 & 117 & 0.0 & 163 & 75.7 & 62.5 \\
\hline Solvent only & 37.9 & 70.9 & 60.2 & 81.3 & 72.5 & 66.5 & 50.3 \\
\hline Solvent only & 19.4 & 62.9 & 52.6 & 0.0 & 50.4 & 56.9 & 42.9 \\
\hline $\begin{array}{c}\text { Sample } \\
\text { component } \\
\text { used }\end{array}$ & $\begin{array}{l}\text { 3,5-Dichloro- } \\
\text { aniline }\end{array}$ & $\begin{array}{l}\text { 4,4'-Dichloro- } \\
\text { benzophenone }\end{array}$ & Dimethoate & $\begin{array}{l}\text { E-Dimetho- } \\
\text { morph }\end{array}$ & $\begin{array}{l}\text { Z-Dimetho- } \\
\text { morph }\end{array}$ & $\begin{array}{l}\text { Disulfoton } \\
\text { sulfone }\end{array}$ & $\alpha$-Endosulfan \\
\hline $\begin{array}{l}1 \text { clean used field } \\
\text { PUF }\end{array}$ & 83.1 & 76.7 & 75.6 & 146 & 136 & 79.2 & 94.5 \\
\hline Solvent only & 62.8 & 56.1 & 59.8 & 68.3 & 68.6 & 64.3 & 80.7 \\
\hline Solvent only & 52.8 & 62.3 & 43.4 & 61.7 & 56.9 & 51.0 & 73.0 \\
\hline $\begin{array}{l}\text { Sample } \\
\text { component } \\
\text { used }\end{array}$ & Fenthion & $\begin{array}{l}\text { Fenthion } \\
\text { sulfone }\end{array}$ & $\begin{array}{l}\text { Fenthion } \\
\text { sulfone } \\
\text { oxygen } \\
\text { analog } \\
\end{array}$ & $\begin{array}{l}\text { Fenthion } \\
\text { sulfoxide }\end{array}$ & $\begin{array}{l}\text { Flumetralin } \\
\text { (estimated) }\end{array}$ & $\begin{array}{c}\text { Fonofos } \\
\text { oxygen } \\
\text { analog } \\
\text { (estimated) }\end{array}$ & $\begin{array}{l}\text { Iprodione } \\
\text { (estimated) }\end{array}$ \\
\hline $\begin{array}{l}1 \text { clean used field } \\
\text { PUF }\end{array}$ & 81.6 & 93.5 & 71.8 & 48.7 & 77.0 & 45.8 & 70.2 \\
\hline Solvent only & 34.0 & 72.5 & 44.4 & 67.8 & 21.7 & 37.3 & 42.5 \\
\hline Solvent only & 29.4 & 53.6 & 36.8 & 58.9 & 17.5 & 33.0 & 142 \\
\hline $\begin{array}{c}\text { Sample } \\
\text { component } \\
\text { used }\end{array}$ & Isofenphos & $\begin{array}{l}\text { Malaoxon } \\
\text { (estimated) }\end{array}$ & Methidathion & $\begin{array}{l}\text { 1,4-Naphtho- } \\
\text { quinone } \\
\text { (estimated) }\end{array}$ & Myclobutanil & Oxyfluorfen & $\begin{array}{c}\text { Paraoxon- } \\
\text { ethyl }\end{array}$ \\
\hline $\begin{array}{l}1 \text { clean used field } \\
\text { PUF }\end{array}$ & 84.6 & 95.2 & 80.7 & 26.7 & 78.4 & 97.0 & 88.0 \\
\hline Solvent only & 56.0 & 52.5 & 57.2 & 13.8 & 54.2 & 59.1 & 44.3 \\
\hline Solvent only & 39.8 & 55.6 & 59.8 & 17.7 & 35.8 & 69.3 & 55.4 \\
\hline
\end{tabular}


Table 25B. Quality-control results for pesticide laboratory reagent spikes using method Airpest002 for samples collected at the Sweetwater Reservoir atmospheric site, San Diego County, California.-Continued

[The site identification number is 324141117001601. Concentrations are given in percent recovered. PUF, polyurethane foam plug; NS, not spiked]

\begin{tabular}{|c|c|c|c|c|c|c|c|}
\hline $\begin{array}{c}\text { Sample } \\
\text { component } \\
\text { used }\end{array}$ & $\begin{array}{c}\text { Paraoxon- } \\
\text { methyl } \\
\text { (estimated) }\end{array}$ & Profenofos & Prometryn & Propetamphos & cis-Propiconazole & $\begin{array}{c}\text { trans- } \\
\text { Propiconazole }\end{array}$ & $\begin{array}{c}\text { O-ethyl-O- } \\
\text { methyl-S- } \\
\text { Propyl- } \\
\text { phosphor- } \\
\text { thioate }\end{array}$ \\
\hline $\begin{array}{l}1 \text { clean used field } \\
\text { PUF }\end{array}$ & 49.2 & 90.2 & 97.0 & 93.8 & 124 & 109 & 89.4 \\
\hline Solvent only & 28.0 & 60.0 & 71.9 & 70.9 & 59.2 & 71.4 & 59.5 \\
\hline Solvent only & 0.00 & 52.1 & 48.8 & 46.4 & 40.0 & 55.6 & 48.7 \\
\hline $\begin{array}{c}\text { Sample } \\
\text { component } \\
\text { used }\end{array}$ & Sulfotepp & Tebupirimphos & $\begin{array}{c}\text { Tebupirimphos } \\
\text { oxygen } \\
\text { analog }\end{array}$ & Tefluthrin & $\begin{array}{l}\text { Temephos } \\
\text { (estimated) }\end{array}$ & $\begin{array}{c}\text { Terbufos } \\
\text { oxygen analog } \\
\text { sulfone } \\
\text { (estimated) } \\
\end{array}$ & Terbuthylazine \\
\hline $\begin{array}{l}1 \text { clean used field } \\
\text { PUF }\end{array}$ & 89.4 & 94.0 & 84.9 & 100 & 134.0 & 85.3 & 99.1 \\
\hline Solvent only & 59.5 & 68.8 & 56.7 & 77.8 & 61.4 & 47.4 & 81.7 \\
\hline Solvent only & 48.7 & 59.2 & 55.6 & 80.1 & 0.00 & 45.3 & 57.9 \\
\hline $\begin{array}{c}\text { Sample } \\
\text { component } \\
\text { used }\end{array}$ & Tribufos & $\begin{array}{c}\text { 3-Trifluoromethyl- } \\
\text { aniline } \\
\text { (estimated) }\end{array}$ & $\begin{array}{l}\text { Diazinon-d10, } \\
\text { surrogate } \\
\text { (percent) }\end{array}$ & $\begin{array}{r}\alpha-H C H-d 6, \\
\text { surrogate } \\
\text { (percent) } \\
\end{array}$ & & & \\
\hline $\begin{array}{l}1 \text { clean used field } \\
\text { PUF }\end{array}$ & 88.4 & 45.3 & 78.8 & 89.8 & & & \\
\hline Solvent only & 54.7 & 49.3 & 125 & 130 & & & \\
\hline Solvent only & 46.3 & 43.9 & 57.6 & 49.8 & & & \\
\hline
\end{tabular}


Table 26A. Quality-control laboratory blank results using pesticides method Airpest003 for air samples collected at the Sweetwater Reservoir atmospheric site, San Diego County, California.

[The site identification number is 324141117001601 . Concentrations are given in nanograms per cubic meter $\left(\mathrm{ng} / \mathrm{m}^{3}\right)$ unless noted. Sample volume is assumed to be $315 \mathrm{~m}^{3}$. GFF, glass fiber filter; PSE, pressurized solvent extraction; PUF, polyurethane foam plug; —, compound not detected at a concentration above laboratory reporting level]

\begin{tabular}{cllccccc}
\hline $\begin{array}{c}\text { Sample } \\
\text { date } \\
\text { (mm/dd/yyyy) }\end{array}$ & $\begin{array}{c}\text { Quality } \\
\text { control } \\
\text { sample type }\end{array}$ & $\begin{array}{c}\text { Sample } \\
\text { components } \\
\text { spiked }\end{array}$ & $\begin{array}{c}\text { Sample } \\
\text { preparation } \\
\text { set number }\end{array}$ & $\begin{array}{c}\text { Extraction } \\
\text { method }\end{array}$ & $\begin{array}{c}\text { Extract split } \\
\text { before } \\
\text { cleanup }\end{array}$ & $\begin{array}{c}\text { Cleanup } \\
\text { column } \\
\text { used for } \\
\text { pesticides }\end{array}$ & $\begin{array}{c}\text { Acetochlor } \\
\text { Alachlor }\end{array}$ \\
\hline $12 / 16 / 2002$ & GFF Blank & GFF & 02.35009 & PSE & no & C18/ Florisil & - \\
$12 / 16 / 2002$ & PUF Blank & PUF & 02.35009 & PSE & no & C18/ Florisil & - \\
$11 / 19 / 2003$ & GFF Blank & GFF & 03.32308 & PSE & yes & Carbo-prep & - \\
$11 / 19 / 2003$ & PUF Blank & PUF & 03.32308 & PSE & yes & Carbo-prep & - \\
$06 / 03 / 2003$ & Field blank GFF & GFF & 03.32308 & PSE & yes & Carbo-prep & - \\
$06 / 03 / 2003$ & $\begin{array}{c}\text { Field blank top } \\
\text { PUF }\end{array}$ & Top PUF & 03.32308 & PSE & yes & Carbo-prep & - \\
$06 / 03 / 2003$ & $\begin{array}{c}\text { Field blank } \\
\text { bottom PUF }\end{array}$ & Bottom PUF & 03.32308 & PSE & yes & Carbo-prep & - \\
& & & & & & -
\end{tabular}

\begin{tabular}{|c|c|c|c|c|c|c|c|c|}
\hline $\begin{array}{c}\text { Sample } \\
\text { date } \\
\text { (mm/dd/yyyy) }\end{array}$ & Atrazine & $\begin{array}{c}\text { Azinphos- } \\
\text { methyl } \\
\text { (estimated) }\end{array}$ & $\begin{array}{l}\text { Azinphos- } \\
\text { methyl } \\
\text { oxon } \\
\text { (estimated) }\end{array}$ & $\begin{array}{l}\text { Benfluralin } \\
\text { (estimated) }\end{array}$ & $\begin{array}{c}\text { Carbaryl } \\
\text { (estimated) }\end{array}$ & $\begin{array}{c}\text { 2-Chloro-2,6- } \\
\text { diethyl- } \\
\text { acet- } \\
\text { anilide }\end{array}$ & $\begin{array}{c}\text { 4-Chloro-2- } \\
\text { methyl- } \\
\text { phenol }\end{array}$ & Chlorpyri \\
\hline $12 / 16 / 2002$ & - & - & - & - & - & - & - & - \\
\hline $12 / 16 / 2002$ & - & - & - & - & - & - & - & - \\
\hline $11 / 19 / 2003$ & - & - & - & - & - & - & - & - \\
\hline $11 / 19 / 2003$ & - & - & - & - & - & - & - & - \\
\hline 06/03/2003 & - & - & - & - & - & - & - & - \\
\hline 06/03/2003 & - & - & - & - & - & - & - & - \\
\hline 06/03/2003 & - & - & - & - & - & - & - & - \\
\hline
\end{tabular}

\begin{tabular}{|c|c|c|c|c|c|c|c|c|}
\hline $\begin{array}{c}\text { Sample } \\
\text { date } \\
\text { (mm/dd/yyyy) }\end{array}$ & $\begin{array}{l}\text { Chlorpyrifos } \\
\text { oxygen } \\
\text { analog } \\
\text { (estimated) }\end{array}$ & $\begin{array}{l}\text { Cyfluthrin } \\
\text { (estimated) }\end{array}$ & $\begin{array}{c}\text { Cyper- } \\
\text { methrin } \\
\text { (estimated) }\end{array}$ & $\begin{array}{l}\text { Dacthal } \\
\text { (DCPA) }\end{array}$ & $\begin{array}{l}\text { Deethyl- } \\
\text { atrazine }\end{array}$ & $\begin{array}{l}\text { Desulfinyl- } \\
\text { fipronil }\end{array}$ & $\begin{array}{l}\text { Desulfinyl- } \\
\text { fipronil } \\
\text { amide } \\
\text { (estimated) }\end{array}$ & Diazinon \\
\hline $12 / 16 / 2002$ & - & - & - & - & - & - & - & - \\
\hline $12 / 16 / 2002$ & - & - & - & - & - & - & - & - \\
\hline $11 / 19 / 2003$ & - & - & - & - & - & - & - & - \\
\hline 06/03/2003 & - & - & - & - & - & - & - & - \\
\hline 06/03/2003 & - & - & - & - & - & - & - & - \\
\hline
\end{tabular}


Table 26A. Quality-control laboratory blank results using pesticide method Airpest003 for air samples collected at the Sweetwater Reservoir atmospheric site, San Diego County, California.-Continued

[The site identification number is 324141117001601 . Concentrations are given in nanograms per cubic meter $\left(\mathrm{ng} / \mathrm{m}^{3}\right)$ unless noted. Sample volume is assumed to be $315 \mathrm{~m}^{3}$. GFF, glass fiber filter; PSE, pressurized solvent extraction; PUF, polyurethane foam plug; —, compound not detected at a concentration above laboratory reporting level]

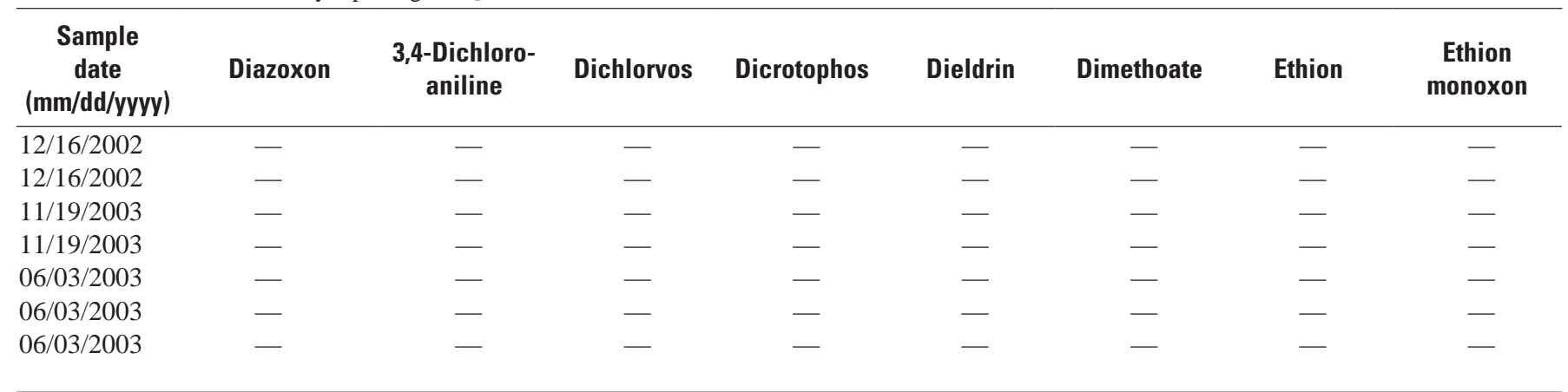

\begin{tabular}{|c|c|c|c|c|c|c|c|c|}
\hline $\begin{array}{c}\text { Sample } \\
\text { date } \\
\text { (mm/dd/yyyy) }\end{array}$ & $\begin{array}{l}\text { 2-Ethyl-6 methyl- } \\
\text { aniline }\end{array}$ & $\begin{array}{l}\text { Fena- } \\
\text { miphos }\end{array}$ & $\begin{array}{l}\text { Fenamiphos } \\
\text { sulfone }\end{array}$ & $\begin{array}{l}\text { Fenamiphos } \\
\text { sulfoxide }\end{array}$ & Fipronil & $\begin{array}{l}\text { Fipronil } \\
\text { sulfide }\end{array}$ & $\begin{array}{l}\text { Fipronil } \\
\text { sulfone }\end{array}$ & Fonofos \\
\hline $12 / 16 / 2002$ & - & - & - & - & - & - & - & - \\
\hline $11 / 19 / 2003$ & - & - & - & - & - & - & - & - \\
\hline $11 / 19 / 2003$ & - & - & - & - & - & - & - & - \\
\hline 06/03/2003 & - & - & - & - & - & - & - & - \\
\hline
\end{tabular}

\begin{tabular}{|c|c|c|c|c|c|c|c|c|}
\hline $\begin{array}{c}\text { Sample } \\
\text { date } \\
\text { (mm/dd/yyyy) }\end{array}$ & $\begin{array}{l}\text { Fonofos } \\
\text { oxygen } \\
\text { analog }\end{array}$ & Hexazinone & Iprodione & $\begin{array}{c}\text { Isofen- } \\
\text { phos }\end{array}$ & Malaoxon & Malathion & Metalaxyl & $\begin{array}{l}\text { Methi- } \\
\text { dathion }\end{array}$ \\
\hline $\begin{array}{l}12 / 16 / 2002 \\
\end{array}$ & - & - & - & - & - & - & - & - \\
\hline $11 / 19 / 2003$ & - & - & - & - & - & - & - & - \\
\hline $11 / 19 / 2003$ & - & - & - & - & - & - & - & - \\
\hline 06/03/2003 & - & - & - & - & - & - & - & - \\
\hline 06/03/2003 & - & - & - & - & - & - & - & - \\
\hline $\begin{array}{c}\text { Sample } \\
\text { date } \\
\text { (mm/dd/yyyy) }\end{array}$ & Metolachlor & Metribuzin & $\begin{array}{l}\text { Myclo- } \\
\text { butanil }\end{array}$ & $\begin{array}{l}\text { Paraoxon- } \\
\text { methyl }\end{array}$ & $\begin{array}{l}\text { Parathion- } \\
\text { methyl }\end{array}$ & $\begin{array}{l}\text { Pendi- } \\
\text { methilan }\end{array}$ & Phorate & $\begin{array}{c}\text { Phorate } \\
\text { oxon }\end{array}$ \\
\hline $11 / 19 / 2003$ & - & - & - & - & - & - & - & - \\
\hline 06/03/2003 & - & - & - & - & - & - & - & - \\
\hline 06/03/2003 & - & - & - & - & - & - & - & - \\
\hline 06/03/2003 & - & - & - & - & - & - & - & - \\
\hline
\end{tabular}


Table 26A. Quality-control laboratory blank results using pesticide method Airpest003 for air samples collected at the Sweetwater Reservoir atmospheric site, San Diego County, California.-Continued

[The site identification number is 324141117001601 . Concentrations are given in nanograms per cubic meter $\left(\mathrm{ng} / \mathrm{m}^{3}\right)$ unless noted. Sample volume is assumed to be $315 \mathrm{~m}^{3}$. GFF, glass fiber filter; PSE, pressurized solvent extraction; PUF, polyurethane foam plug; - , compound not detected at a concentration above laboratory reporting level]

\begin{tabular}{|c|c|c|c|c|c|c|c|c|}
\hline $\begin{array}{c}\text { Sample } \\
\text { date } \\
\text { (mm/dd/yyyy) }\end{array}$ & Prometon & Prometryn & Propyzamide & Simazine & Tebuthiuron & Terbufos & $\begin{array}{l}\text { Terbufos } \\
\text { oxygen } \\
\text { analog } \\
\text { sulfone }\end{array}$ & $\begin{array}{l}\text { Terbuthyl- } \\
\text { azine }\end{array}$ \\
\hline $12 / 16 / 2002$ & - & - & - & - & - & - & - & - \\
\hline $12 / 16 / 2002$ & - & - & - & - & - & - & - & - \\
\hline $06 / 03 / 2003$ & - & - & - & - & - & - & - & - \\
\hline $06 / 03 / 2003$ & - & - & - & - & - & - & - & - \\
\hline $06 / 03 / 2003$ & - & - & - & - & - & - & - & - \\
\hline
\end{tabular}

\begin{tabular}{lccccc}
\hline $\begin{array}{c}\text { Sample } \\
\text { date } \\
\text { (mm/dd/yyyy) }\end{array}$ & cis-Permethrin & $\begin{array}{c}\text { trans- } \\
\text { Permethrin }\end{array}$ & Trifluralin & $\begin{array}{c}\text { Diazinon-d10, } \\
\text { surrogate } \\
\text { (percent) }\end{array}$ & $\begin{array}{c}\boldsymbol{\alpha} \text {-HCH-d6, } \\
\text { surrogate } \\
\text { (percent) }\end{array}$ \\
\hline $12 / 16 / 2002$ & - & - & - & 52.0 & 38.0 \\
$12 / 16 / 2002$ & - & - & - & 62.0 & 55.0 \\
$11 / 19 / 2003$ & - & - & - & 70.8 & 44.6 \\
$11 / 19 / 2003$ & - & - & - & 101 & 93.7 \\
$06 / 03 / 2003$ & - & - & - & 83.8 & 70.8 \\
$06 / 03 / 2003$ & - & - & - & 106 & 99.1 \\
$06 / 03 / 2003$ & - & - & - & 95.2 & 88.7 \\
\hline
\end{tabular}


Table 26B. Quality-control laboratory reagent-spike results using pesticide method Airpest003 for air samples collected at the Sweetwater Reservoir atmospheric site, San Diego County, California.

[The site identification number is 324141117001601 . Values for compounds are in percent recovery. QC, quality control; E, estimated values; na, not applicable; PSE, pressurized solvent extraction; <, compound not detected at a concentration above reporting level]

\begin{tabular}{cccccccrc}
\hline $\begin{array}{c}\text { Sample } \\
\text { date } \\
\text { (mm/dd/yyyy) }\end{array}$ & $\begin{array}{c}\text { QC } \\
\text { sample } \\
\text { type }\end{array}$ & $\begin{array}{c}\text { Sample } \\
\text { components } \\
\text { spiked }\end{array}$ & $\begin{array}{c}\text { Sample } \\
\text { preparation } \\
\text { set number }\end{array}$ & $\begin{array}{c}\text { Extraction } \\
\text { method }\end{array}$ & $\begin{array}{c}\text { Extract split } \\
\text { before } \\
\text { cleanup }\end{array}$ & $\begin{array}{c}\text { Cleanup } \\
\text { column used } \\
\text { for } \\
\text { pesticides }\end{array}$ & Acetochlor & Alachlor \\
\hline $12 / 16 / 2002$ & GFF Spike & GFF & 02.35009 & PSE & no & C18/ Florisil & 66.0 \\
$12 / 16 / 2002$ & PUF Spike & PUF & 02.35009 & PSE & no & C18/ Florisil & 76.0 & E 75.3 84.5 \\
$11 / 19 / 2003$ & GFF Spike & GFF & 03.32308 & PSE & yes & Carbo-prep & 104 \\
$11 / 19 / 2003$ & PUF Spike & PUF & 03.32308 & PSE & yes & Carbo-prep & 115 & 98.1 \\
& & & & & & & 115 &
\end{tabular}

\begin{tabular}{|c|c|c|c|c|c|c|c|c|}
\hline $\begin{array}{c}\text { Sample } \\
\text { date } \\
\text { (mm/dd/yyyy) }\end{array}$ & Atrazine & $\begin{array}{c}\text { Azinphos- } \\
\text { methyl } \\
\text { (estimated) }\end{array}$ & $\begin{array}{l}\text { Azinphos- } \\
\text { methyl } \\
\text { oxon } \\
\text { (estimated) }\end{array}$ & $\begin{array}{l}\text { Benfluralin } \\
\text { (estimated) }\end{array}$ & $\begin{array}{c}\text { Carbaryl } \\
\text { (estimated) }\end{array}$ & $\begin{array}{l}\text { 2-Chloro- } \\
\text { 2,6-diethyl- } \\
\text { acetanilide }\end{array}$ & $\begin{array}{c}\text { 4-Chloro-2- } \\
\text { methyl- } \\
\text { phenol }\end{array}$ & Chlorpyrifos \\
\hline $12 / 16 / 2002$ & 57.3 & $<40$ & 0.0 & 47.9 & 14.8 & 63.6 & E 39.2 & 54.2 \\
\hline $12 / 16 / 2002$ & 59.3 & $<175$ & 0.0 & 57.3 & 69.3 & 67.3 & Е 60.4 & 68.5 \\
\hline $11 / 19 / 2003$ & 110 & 31.7 & 0.0 & 89.3 & 0.0 & 110 & 0.0 & 99.8 \\
\hline
\end{tabular}

\begin{tabular}{|c|c|c|c|c|c|c|c|c|}
\hline $\begin{array}{c}\text { Sample } \\
\text { date } \\
\text { (mm/dd/yyyy) }\end{array}$ & $\begin{array}{c}\text { Chlorpyrifos } \\
\text { oxygen } \\
\text { analog } \\
\text { (estimated) }\end{array}$ & $\begin{array}{l}\text { Cyfluthrin } \\
\text { (estimated) }\end{array}$ & $\begin{array}{c}\text { Cyper- } \\
\text { methrin } \\
\text { (estimated) }\end{array}$ & $\begin{array}{l}\text { Dacthal } \\
\text { (DCPA) }\end{array}$ & $\begin{array}{l}\text { Deethyl- } \\
\text { atriazine }\end{array}$ & $\begin{array}{l}\text { Desulfinyl- } \\
\text { fipronil }\end{array}$ & $\begin{array}{l}\text { Desulfinyl } \\
\text { fipronil } \\
\text { amide } \\
\text { (estimated) }\end{array}$ & Diazinon \\
\hline $12 / 16 / 2002$ & 0.0 & 0.0 & 0.0 & 64.7 & E 59.1 & 62.1 & $<35$ & 57.3 \\
\hline $12 / 16 / 2002$ & 0.0 & 128 & 84.4 & 67.2 & E 56.1 & 70.3 & E 64.1 & 66.0 \\
\hline $11 / 19 / 2003$ & 0.0 & 0.0 & 0.0 & 113 & 74 & 107 & 40.5 & 96.1 \\
\hline
\end{tabular}

\begin{tabular}{ccccccccc}
\hline $\begin{array}{c}\text { Sample } \\
\text { date } \\
\text { (mm/dd/yyyy) }\end{array}$ & $\begin{array}{c}\text { Diazoxon } \\
\text { (estimated) }\end{array}$ & $\begin{array}{c}\text { 3,4-Di- } \\
\text { chloro- } \\
\text { aniline } \\
\text { (estimated) }\end{array}$ & $\begin{array}{c}\text { Dichlorvos } \\
\text { (estimated) }\end{array}$ & $\begin{array}{c}\text { Dicro- } \\
\text { tophos } \\
\text { (estimated) }\end{array}$ & Dieldrin & Dimethoate & $\begin{array}{c}\text { Ethion } \\
\text { Ethionon } \\
\text { (estimated) }\end{array}$ \\
\hline $12 / 16 / 2002$ & 0.0 & 51.9 & 0.0 & 0.0 & 69.3 & E 30.8 & 41.6 & 63.5 \\
$12 / 16 / 2002$ & 48.7 & 59.0 & 6.2 & 0.0 & 67.9 & E 63.7 & 61.6 \\
$11 / 19 / 2003$ & 29.9 & 4.9 & 0.0 & 55.7 & 98.4 & 94.2 & 73.6 & 26.0 \\
$11 / 19 / 2003$ & 110 & 39.2 & 76.1 & 119 & 119 & 78.9 & 144 \\
\end{tabular}

\begin{tabular}{|c|c|c|c|c|c|c|c|c|}
\hline $12 / 16 / 2002$ & E 53.7 & 0.0 & 0.0 & 0.0 & 36.3 & 60.2 & 55.5 & 50.2 \\
\hline $12 / 16 / 2002$ & E 46.5 & 49.8 & 0.0 & 0.0 & 73.4 & 74.9 & 23.1 & 57.8 \\
\hline $11 / 19 / 2003$ & E 13.9 & 59.0 & 46.6 & 76.8 & 41.6 & 98.9 & 52.6 & 92.3 \\
\hline
\end{tabular}


Table 26B. Quality-control laboratory reagent-spike results using pesticide method Airpest003 for air samples collected at the Sweetwater Reservoir atmospheric site, San Diego County, California.-Continued

[The site identification number is 324141117001601 . Values for compounds are in percent recovery. QC, quality control; E, estimated values; na, not applicable; PSE, pressurized solvent extraction; <, compound not detected at a concentration above reporting level]

\begin{tabular}{|c|c|c|c|c|c|c|c|c|}
\hline $\begin{array}{c}\text { Sample } \\
\text { date } \\
\text { (mm/dd/yyyy) }\end{array}$ & $\begin{array}{c}\text { Fonofos } \\
\text { oxygen } \\
\text { analog } \\
\text { (estimated) }\end{array}$ & $\begin{array}{l}\text { Hexazinone } \\
\text { (estimated) }\end{array}$ & $\begin{array}{l}\text { Iprodione } \\
\text { (estimated) }\end{array}$ & Isofenphos & $\begin{array}{l}\text { Malaoxon } \\
\text { (estimated) }\end{array}$ & $\begin{array}{l}\text { Malathion } \\
\text { (estimated) }\end{array}$ & Metalaxyl & Methidathion \\
\hline$\overline{12 / 16 / 2002}$ & 0.0 & 0.0 & 0.0 & 42.8 & 0.0 & 0.0 & 0.0 & 43.3 \\
\hline $12 / 16 / 2002$ & 23.1 & 0.0 & 159 & 71.4 & 56.2 & 68.3 & 0.0 & 82.7 \\
\hline $11 / 19 / 2003$ & 41.2 & 78.8 & 0.0 & 99.0 & 0.0 & 0.0 & 116 & 73 \\
\hline $\begin{array}{c}\text { Sample } \\
\text { date } \\
\text { (mm/dd/yyyy) }\end{array}$ & Metolachlor & Metribuzin & Myclobutanil & $\begin{array}{c}\text { Paraoxon- } \\
\text { methyl } \\
\text { (estimated) }\end{array}$ & $\begin{array}{c}\text { Parathion- } \\
\text { methyl }\end{array}$ & $\begin{array}{l}\text { Pendi- } \\
\text { methilan }\end{array}$ & $\begin{array}{c}\text { Phorate } \\
\text { (estimated) }\end{array}$ & $\begin{array}{c}\text { Phorate } \\
\text { oxon } \\
\text { (estimated) }\end{array}$ \\
\hline $12 / 16 / 2002$ & 58.0 & 43.8 & 0.0 & 0.0 & E 56.0 & 49.6 & 40.7 & 0.0 \\
\hline $12 / 16 / 2002$ & 66.9 & 64.3 & 52.9 & 30 & E 70.6 & 67.2 & 77.4 & 88.8 \\
\hline
\end{tabular}

\begin{tabular}{|c|c|c|c|c|c|c|c|c|}
\hline $\begin{array}{c}\text { Sample } \\
\text { date } \\
\text { (mm/dd/yyyy) }\end{array}$ & Prometon & Prometryn & Propyzamide & Simazine & $\begin{array}{l}\text { Tebuthiuron } \\
\text { (estimated) }\end{array}$ & Terbufos & $\begin{array}{l}\text { Terbufos } \\
\text { oxygen } \\
\text { analog } \\
\text { sulfone } \\
\text { (estimated) }\end{array}$ & $\begin{array}{l}\text { Terbuthyl- } \\
\text { azine }\end{array}$ \\
\hline $12 / 16 / 2002$ & 44.3 & 45.4 & 63.6 & 66.6 & 0.0 & $<125$ & 0.0 & 62.2 \\
\hline $12 / 16 / 2002$ & 55.3 & 72.7 & 71.4 & 71.8 & 32 & 134 & 0.0 & 67.8 \\
\hline $11 / 19 / 2003$ & 81.9 & 84.0 & 66.4 & 104 & 0.0 & 71.2 & 0.0 & 110 \\
\hline $\begin{array}{c}\text { Sample } \\
\text { date } \\
\text { (mm/dd/yyyy) }\end{array}$ & $\begin{array}{c}\text { cis- } \\
\text { Permethrin }\end{array}$ & $\begin{array}{c}\text { trans- } \\
\text { Perrmethrin }\end{array}$ & $\begin{array}{l}\text { Trifluralin } \\
\text { (estimated) }\end{array}$ & $\begin{array}{c}\text { Diazinon-dno, } \\
\text { surrogate } \\
\text { (percent) }\end{array}$ & $\begin{array}{l}\alpha-\mathrm{HCH}-\alpha 6, \\
\text { surrogate } \\
\text { (percent) }\end{array}$ & & & \\
\hline$\overline{12 / 16 / 2002}$ & 67.0 & 59.2 & 50.1 & 63.9 & 46.4 & & & \\
\hline
\end{tabular}


Table 27A. Quality-control laboratory reagent-water blank results for the special study of pharmaceutical compounds in the Sweetwater Reservoir watershed, San Diego County, California.

[LRL, Laboratory reporting level; —, compound not detected at a concentration above laboratory reporting level; mL, milliliter. All values were reported as micrograms per liter $(\mu \mathrm{g} / \mathrm{L})$ unless noted]

\begin{tabular}{|c|c|c|c|c|c|c|}
\hline $\begin{array}{c}\text { Date } \\
\text { (mm/dd/yyyy) }\end{array}$ & $\begin{array}{c}\text { Sample } \\
\text { volume (mL) }\end{array}$ & $\begin{array}{l}\text { Acetaminophen } \\
(62000)\end{array}$ & $\begin{array}{l}\text { Albuterol } \\
(62020)\end{array}$ & $\begin{array}{l}\text { Caffeine } \\
\text { (50305) }\end{array}$ & $\begin{array}{c}\text { Carbamazapine } \\
\text { (62793) }\end{array}$ & $\begin{array}{l}\text { Codeine } \\
\text { (62002) }\end{array}$ \\
\hline [LRL] & & {$[0.22]$} & [0.19] & {$[0.19]$} & [0.18] & {$[0.20]$} \\
\hline $03 / 20 / 2002$ & 918 & - & - & - & - & - \\
\hline 06/11/2002 & 901 & - & - & - & - & - \\
\hline $12 / 12 / 2002$ & 912 & - & - & - & - & - \\
\hline $02 / 11 / 2003$ & 944 & - & - & - & - & - \\
\hline 04/09/2003 & 933 & - & - & - & - & - \\
\hline 08/20/2003 & 921 & - & - & - & - & - \\
\hline $\begin{array}{c}\text { Date } \\
\text { (mm/dd/yyyy) }\end{array}$ & $\begin{array}{l}\text { Cotinine } \\
\text { (62005) }\end{array}$ & $\begin{array}{l}\text { Dehydro- } \\
\text { nifedipine } \\
(62004)\end{array}$ & $\begin{array}{l}\text { 1,7 Dimethyl- } \\
\text { xanthine } \\
(62030)\end{array}$ & $\begin{array}{l}\text { Diltiazem } \\
\text { (62008) }\end{array}$ & $\begin{array}{l}\text { Diphen- } \\
\text { hydramine } \\
(62796)\end{array}$ & $\begin{array}{l}\text { Sulfameth- } \\
\text { oxazole } \\
\text { (62021) }\end{array}$ \\
\hline [LRL] & {$[0.19]$} & {$[0.20]$} & [0.22] & {$[0.18]$} & {$[0.18]$} & {$[0.21]$} \\
\hline $03 / 20 / 2002$ & - & - & - & - & - & - \\
\hline 06/11/2002 & - & - & - & 0.001 & - & - \\
\hline $12 / 12 / 2002$ & - & - & - & 0.002 & 0.003 & - \\
\hline $02 / 11 / 2003$ & - & - & - & - & 0.002 & - \\
\hline 04/09/2003 & - & - & - & - & - & - \\
\hline 08/20/2003 & - & - & - & - & - & - \\
\hline $\begin{array}{c}\text { Date } \\
\text { (mm/dd/yyyy) }\end{array}$ & $\begin{array}{l}\text { Thiabendazole } \\
\text { (62081) }\end{array}$ & $\begin{array}{l}\text { Trimethoprim } \\
\text { (62023) }\end{array}$ & $\begin{array}{l}\text { Warfarin } \\
\text { (62024) }\end{array}$ & $\begin{array}{l}\text { Ethylnico- } \\
\text { tinate-d4, } \\
\text { surrograte } \\
\text { (95571) } \\
\text { (percent) }\end{array}$ & & \\
\hline [LRL] & {$[0.18]$} & {$[0.17]$} & [0.19] & & & \\
\hline $03 / 20 / 2002$ & - & - & - & 105 & & \\
\hline 06/11/2002 & - & 0.002 & - & 85 & & \\
\hline $12 / 12 / 2002$ & - & - & - & 73 & & \\
\hline $02 / 11 / 2003$ & - & - & - & 74 & & \\
\hline 04/09/2003 & - & - & - & 74 & & \\
\hline $08 / 20 / 2003$ & - & - & - & 96 & & \\
\hline
\end{tabular}


Table 27B. Quality-control laboratory reagent-water spike results for the special study of pharmaceutical compounds in the Sweetwater Reservoir watershed, San Diego County, California.

[LRL, Laboratory reporting level; $\mathrm{mL}$, milliliter. All values were reported as percent recovered]

\begin{tabular}{|c|c|c|c|c|c|c|}
\hline $\begin{array}{c}\text { Date } \\
\text { (mm/dd/yyyy) }\end{array}$ & $\begin{array}{c}\text { Sample } \\
\text { volume } \\
\text { (mL) }\end{array}$ & $\begin{array}{l}\text { Acetaminophen } \\
(62000)\end{array}$ & $\begin{array}{c}\text { Albuterol } \\
(62020)\end{array}$ & $\begin{array}{c}\text { Caffeine } \\
\text { (50305) }\end{array}$ & $\begin{array}{c}\text { Carbamazapine } \\
(62793)\end{array}$ & $\begin{array}{l}\text { Codeine } \\
\text { (62002) }\end{array}$ \\
\hline [LRL] & & {$[0.22]$} & {$[0.19]$} & {$[0.19]$} & {$[0.18]$} & {$[0.20]$} \\
\hline $03 / 20 / 2002$ & 918 & 85 & 81 & 100 & 70 & 92 \\
\hline $06 / 11 / 2002$ & 872 & 71 & 76 & 84 & 67 & 69 \\
\hline $12 / 12 / 2002$ & 930 & 66 & 88 & 89 & 89 & 180 \\
\hline $02 / 11 / 2003$ & 952 & 68 & 87 & 98 & 86 & 195 \\
\hline 04/092003 & 933 & 54 & 65 & 82 & 70 & 139 \\
\hline $08 / 20 / 2003$ & 912 & 85 & 63 & 81 & 103 & 101 \\
\hline $\begin{array}{c}\text { Date } \\
\text { (mm/dd/yyyy) }\end{array}$ & $\begin{array}{c}\text { Cotinine } \\
\text { (62005) }\end{array}$ & $\begin{array}{l}\text { Dehydro- } \\
\text { nifedipine } \\
(62004)\end{array}$ & $\begin{array}{l}\text { 1,7 Dimethyl- } \\
\text { xanthine } \\
(62030)\end{array}$ & $\begin{array}{c}\text { Diltiazem } \\
(62008)\end{array}$ & $\begin{array}{l}\text { Diphen- } \\
\text { hydramine } \\
\text { (62796) }\end{array}$ & $\begin{array}{l}\text { Sulfameth- } \\
\text { oxazole } \\
\text { (62021) }\end{array}$ \\
\hline [LRL] & {$[0.19]$} & {$[0.20]$} & {$[0.22]$} & {$[0.18]$} & {$[0.18]$} & {$[0.21]$} \\
\hline $03 / 20 / 2002$ & 78 & 95 & 188 & 59 & 60 & 68 \\
\hline $06 / 11 / 2002$ & 74 & 71 & 119 & 46 & 57 & 50 \\
\hline $12 / 12 / 2002$ & 92 & 90 & 80 & 64 & 61 & 31 \\
\hline $02 / 11 / 2003$ & 92 & 77 & 90 & 35 & 56 & 45 \\
\hline $04 / 092003$ & 75 & 61 & 76 & 30 & 2 & 11 \\
\hline $08 / 20 / 2003$ & 109 & 100 & 86 & 68 & 80 & 98 \\
\hline
\end{tabular}

\begin{tabular}{ccccc}
\hline $\begin{array}{c}\text { Date } \\
\text { (mm/dd/yyyy) }\end{array}$ & $\begin{array}{c}\text { Thiabendazole } \\
\mathbf{( 6 2 0 8 1 )}\end{array}$ & $\begin{array}{c}\text { Trimethoprim } \\
\mathbf{( 6 2 0 2 3 )}\end{array}$ & $\begin{array}{c}\text { Warfarin } \\
\mathbf{( 6 2 0 2 4 )}\end{array}$ & $\begin{array}{c}\text { Ethylnico- } \\
\text { tinate-d4, } \\
\text { surrograte } \\
\text { (95571) } \\
\text { (percent) }\end{array}$ \\
\hline [LRL] & {$[\mathbf{0 . 1 8}]$} & {$[\mathbf{0 . 1 7}]$} & {$[\mathbf{0 . 1 9}]$} & \\
\hline $03 / 20 / 2002$ & 84 & 75 & 79 & 105 \\
$06 / 11 / 2002$ & 76 & 69 & 56 & 85 \\
$12 / 12 / 2002$ & 75 & 86 & 77 & 73 \\
$02 / 11 / 2003$ & 65 & 86 & 62 & 74 \\
$04 / 092003$ & 68 & 74 & 43 & 74 \\
$08 / 20 / 2003$ & 17 & 68 & 88 & 96 \\
\hline
\end{tabular}






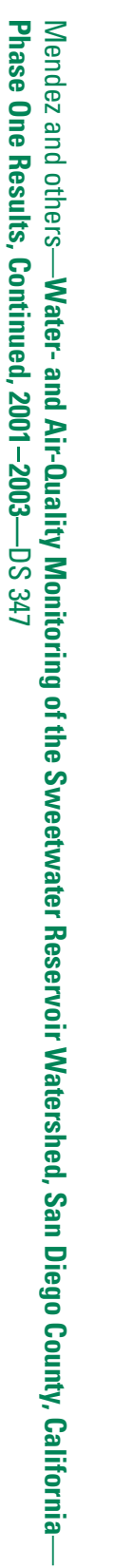

RI
E్
怘 